University of Louisville

ThinkIR: The University of Louisville's Institutional Repository

$5-2021$

\title{
Harnessing the power of trained immunity in the setting of pancreatic cancer: a novel mechanism of immune trafficking and tumor control.
}

Anne Elena Geller

University of Louisville

Follow this and additional works at: https://ir.library.louisville.edu/etd

Part of the Biological Phenomena, Cell Phenomena, and Immunity Commons, Immunotherapy Commons, Medical Immunology Commons, Neoplasms Commons, and the Oncology Commons

\section{Recommended Citation}

Geller, Anne Elena, "Harnessing the power of trained immunity in the setting of pancreatic cancer: a novel mechanism of immune trafficking and tumor control." (2021). Electronic Theses and Dissertations. Paper 3593.

https://doi.org/10.18297/etd/3593

This Doctoral Dissertation is brought to you for free and open access by ThinkIR: The University of Louisville's Institutional Repository. It has been accepted for inclusion in Electronic Theses and Dissertations by an authorized administrator of ThinkIR: The University of Louisville's Institutional Repository. This title appears here courtesy of the author, who has retained all other copyrights. For more information, please contact thinkir@louisville.edu. 
HARNESSING THE POWER OF TRAINED IMMUNITY IN THE SETTING OF

PANCREATIC CANCER: A NOVEL MECHANISM OF IMMUNE TRAFFICKING

AND TUMOR CONTROL

Anne Elena Geller

B.S., University of Pennsylvania 2013

M.S., Tufts University 2014

\begin{abstract}
A Dissertation
Submitted to the faculty of the

School of Medicine of the University of Louisville

In partial fulfillment of the requirements

for the Degree of

Doctor of Philosophy

In Microbiology and Immunology
\end{abstract}

Department of Microbiology and Immunology

University of Louisville

May, 2021 
Copyright 2021 by Anne E. Geller

All Rights Reserved 

HARNESSING THE POWER OF TRAINED IMMUNITY IN THE SETTING OF

PANCREATIC CANCER: A NOVEL MECHANISM OF IMMUNE TRAFFICKING

AND TUMOR CONTROL

Anne Elena Geller

B.S., University of Pennsylvania 2013

M.S., Tufts University 2014

A dissertation approved on

April 5, 2021

By the following Dissertation Committee

Jun Yan, M.D., Ph.D.

Dissertation director

Nejat Egilmez, Ph.D.

Haribabu Bodduluri, Ph.D.

Venkatakrishna Jala, Ph.D. 


\section{DEDICATION}

This dissertation is dedicated to my parents

Dr. Clint B. Geller

and

Dr. Maria E. Magone

Their unconditional love and support is the greatest gift there is. All that I am or hope to be, I owe to them. 


\section{ACKNOWLEDGEMENTS}

I would first like to thank my primary mentor, Dr. Jun Yan for his guidance, his patience, for challenging me and for sharing his infectious love for science and immunology. Under his direction I have grown enormously as a person, a future physician and as a scientist. He is an excellent scientist and has made me into an independent and curious investigator and for that I will be always grateful. I would like to thank my committee members Dr. Haribabu Bodduluri, Dr. Venkatakrishna Jala, and Dr. Nejat Egilmez. They have each provided me with valuable support and direction throughout this journey. I would like to thank the members of the Yan lab over the years who I have learned from and collaborated with, especially Dr. Chuanlin Ding, Xiaoling Hu, Rejeena Shrestha and Dr. Samantha Morrissey who have provided friendship and scientific guidance. I would also like to thank Dr. Hixun Guo, Dr. Kalina Andreeva and Dr. Julia Chariker who have each been an enormous help in many studies and who I have enjoyed collaborating with. Dr. Kelly M McMasters and Dr. Robert Martin provided valuable clinical guidance and feedback for which I am grateful. I would also like to thank Dr. Russel Salter and Victoria King from the $\mathrm{MD} / \mathrm{PhD}$ program at the University of Louisville for their guidance and encouragement over the last 6 years.

A most important thank you goes to my parents, Dr. Clint Geller and Dr. Maria Magone. Their sacrifices to help me achieve my dreams have not been small. They instilled in me a love for science at a young age, provided a nurturing and unwavering support system, and gifted me with a sincere belief that I am capable of achieving anything. Making 
them proud is the greatest and most profound honor. A heartfelt thank you also goes to my partner Michael Liebler. Over the last 6 years he has supported and encouraged me through all of the ups and downs of graduate school. He has been a shoulder to cry on, a chef on the numerous occasions when I worked late, a professional cat photographer, and a source of endless joy, laughter, kindness and stability on this journey. Finally, I would like to thank my friends, past teachers, mentors and colleagues from all walks of life who have supported my dreams and who have encouraged me along the way. My achievements are but a reflection of the support I have around me. To quote my father's 1979 dissertation, "it boggles the mind how one lacking companionship of such quality can long survive the incredible ordeal of graduate work. 


\begin{abstract}
HARNESSING THE POWER OF TRAINED IMMUNITY IN THE SETTING OF PANCREATIC CANCER: A NOVEL MECHANISM OF IMMUNE TRAFFICKING AND TUMOR CONTROL
\end{abstract}

Anne E. Geller

April 5, 2021

Despite the success of immunotherapy in many types of cancer, pancreatic adenocarcinoma (PDAC) has yet to benefit. Innate immune cells are critical to antitumor immunosurveillance and recent studies have revealed that these populations possess a form of memory, termed trained innate immunity, which occurs through transcriptomic, epigenetic, and metabolic reprograming. Though trained innate immunity has mostly been investigated in the context of infection, the induction of trained innate immunity could also protect against tumors, and specifically pancreatic tumors. Here, we demonstrate that yeast-derived particulate $\beta$-glucan, a known inducer of trained immunity, traffics to the pancreas following IP administration. This causes a CCR2-dependent influx of newly characterized monocytes/macrophages to the pancreas which display features of trained immunity. These trained cells can be activated upon exposure to tumor cells and tumor- 
derived factors, and show enhanced phagocytosis and ROS-mediated cytotoxicity against pancreatic tumors. In orthotopic models of pancreatic cancer, mice trained with $\beta$-glucan show reduced tumor burden and prolonged survival which is further enhanced when combined with anti-PD-L1 immunotherapy. Cumulatively, these findings not only add novel characterization to the dynamic mechanisms, scope and localization of peripheral trained immunity, but also identify a direct application of trained immunity to cancer that can be utilized directly within the pancreas to reprogram the immunologically cold tumor microenvironment of PDAC. 


\section{TABLE OF CONTENTS}

\section{PAGE}

SIGNATURE PAGE

ii

DEDICATION

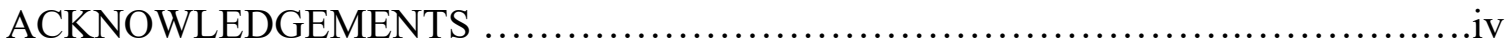

ABSTRACT

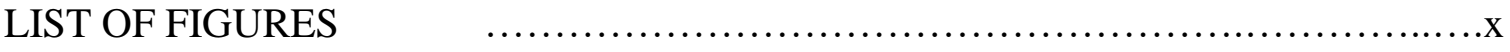

INTRODUCTION $\quad$..........................................................

General Introduction .......................................

Myeloid Cells in the Tumor Microenvironment................3

The Tumor Microenvironment of Pancreatic Cancer...........8

Trained Innate Immunity .................................11

CHAPTER I: The trafficking and biodistribution of $\beta$-glucan ........................20

Introduction......................................... 20

Results...............................................21

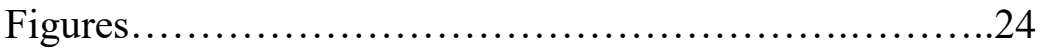

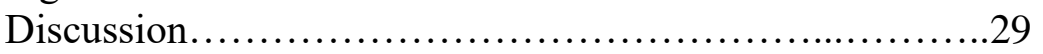

CHAPTER II: $\beta$-glucan trafficking to the pancreas incites an influx of innate immune cells to the pancreas that show a phenotype of trained immunity..........32

Introduction................................................ 32

Results.................................................... 33

Figures...........................................42

Discussion.............................................46

CHAPTER III: Trafficking of immune cells to the pancreas due to $\beta$-glucan

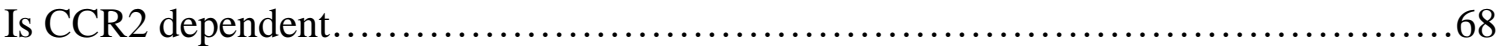

Introduction..........................................68

Results...............................................69

Figures.............................................. 71

Discussion............................................. 77 
CHAPTER IV: WGP as an anti-tumor agent in pancreatic cancer......................79

Introduction........................................... 79

Results.............................................. 80

Figures............................................. 88

Discussion..........................................104

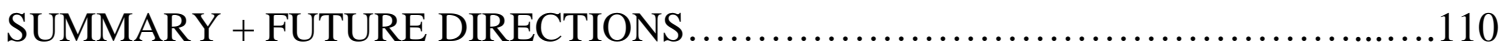

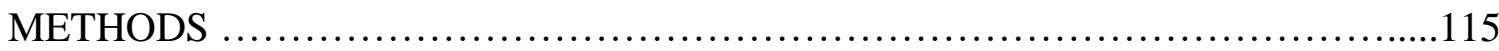

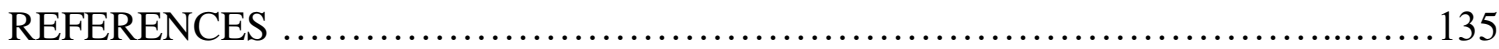

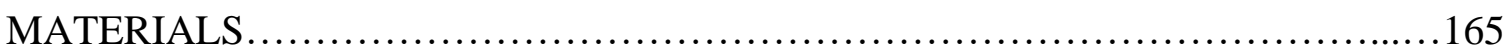

CURRICULUM VITA........................................................ 173 


\section{LIST OF FIGURES}

\section{INTRODUCTION}

Figure I.1: Schematic of trained immunity ........................................ 14

\section{CHAPTER I}

Figure 1.1: IP DTAF WGP preferentially accumulates in the pancreas................24

Figure 1.2: Analysis of WGP trafficking using radio-labeled ${ }^{89} \mathrm{Zr}-\mathrm{WGP} \ldots \ldots \ldots \ldots \ldots . .25$

Figure 1.3: Dectin-1 regulates the trafficking of WGP to the pancreas.................26

Figure 1.4: A polystyrene bead the same size as WGP does not accumulate

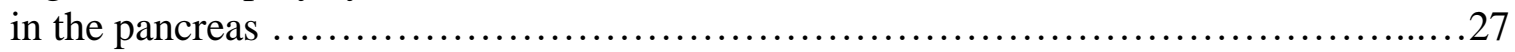

Figure 1.5: Confocal microscopy of in vivo phagocytosis of DTAF-WGP...............27

Figure 1.6: There is a transient flux of macrophages between the peritoneal cavity and the pancreas that is utilized to deliver WGP to the pancreas..................28

\section{CHAPTER II}

Figure 2.1: IP WGP traffics to the bone marrow and causes an expansion

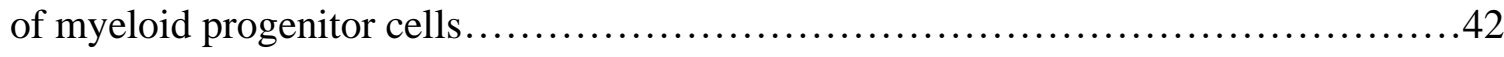

Figure 2.2: IP WGP causes an influx of CD11b+ myeloid cells into the pancreas .......43

Figure 2.3: A transient influx of diverse myeloid cell subtypes occurs after

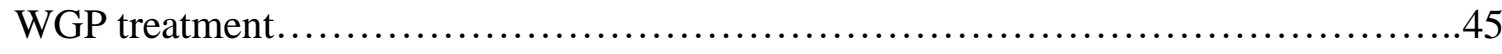

Figure 2.4: The trafficking of immune cells into the pancreas is dose dependent.........46

Figure 2.5: The influx of immune cells is not C57BL/6 specific ......................46

Figure 2.6: The WGP-driven influx of immune cells does not harm the pancreas .......47 
Figure 2.7: H+E imaging of PBS and WGP treated pancreases....................48

Figure 2.8: Myeloid cells in the pancreas display features of trained immunity..........49

Figure 2.9: Enriched CD11b+ pancreatic cells show a phenotype of trained immunity .50

Figure 2.10: The induction of trained immunity in germ-free mice. .51

Figure 2.11: RNA-Seq analysis of PBS versus WGP treated pancreatic CD11b+ cells................................................. 52

Figure 2.12: CyTOF analysis of the pancreas of PBS vs WGP treated mice..............53

Figure 2.13: Heatmap of the median scaled expression of the CyTOF markers in each cluster determined from CyTOF workflow.... 54

Figure 2.14: Functional characterization of pancreatic CD11b+cells using CyTOF

Figure 2.15: Training of pancreatic myeloid cells is not dependent on T-cells .56

Figure 2.16: Training of pancreatic myeloid cells is not dependent on NK cells.

Figure 2.17: Adaptive immune responses to not involved in the training of pancreatic CD11b+ myeloid cells .58

Figure 2.18: Training of pancreatic myeloid cells is not dependent on granulocytes

Figure 2.19: Single-cell RNA-Seq showing the immune cell phenotype 3 and 7 days following IP WGP.

Figure 2.20: scRNA-Seq identifies CSF1R+ myeloid populations that are increased following WGP treatment.

Figure 2.21: Classification of differentially regulated myeloid populations in the pancreas using scRNa-Seq

Figure 2.22: Characterization of differentially regulated myeloid populations in the pancreas using scRNa-Seq

\section{CHAPTER III}

Figure 3.1: Chemokines and cytokines upregulated by WGP treatment .71 
Figure 3.2: CCR2/CCL2 signaling is increased due to WGP treatment

Figure 3.3: Proliferation of CCR2 + cells in the pancreas......................... 74

Figure 3.4: CCR2+ cells in the pancreas are trained due to WGP .....................74

Figure 3.5: cellular influx and training of pancreatic innate myeloid

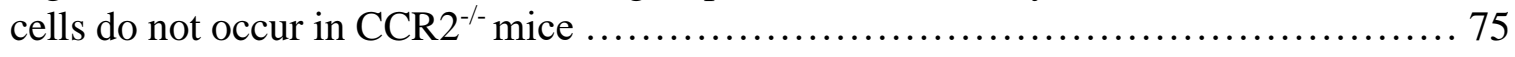

Figure 3.6: Pancreatic myeloid cells are trained by 24 and 48 hours post-WGP..........76

\section{CHAPTER IV}

Figure 4.1: Schematic of the anti-tumor effects of WGP-induced trained

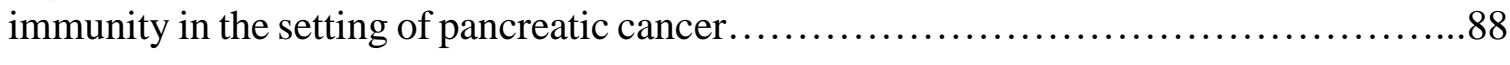

Figure 4.2: Tumor conditioned media elicits a trained response in vitro...............89

Figure 4.3: Tumor conditioned media elicits a trained response ex vivo...............90

Figure 4.4: MIF secreted by tumor cells elicits the trained response.................. 91

Figure 4.5: Recombinant HMGB1 elicits the trained response.....................91

Figure 4.6: WGP treatment positively regulates genes related to phagocytic cellular processes.

Figure 4.7: WGP training increases phagocytosis of a pHrodo Green Staph Aureus particle............................................ 93

Figure 4.8: WGP training increases phagocytosis of KPC tumor cells................94

Figure 4.9: WGP treatment enhances cytotoxicity of myeloid cells against tumor cells

Figure 4.10: WGP treatment decreases tumor size in an orthotopic model of pancreatic cancer............................................... 96

Figure 4.11: Immune phenotyping of PBS vs WGP treated pancreatic KPC tumors.......97

Figure 4.12: Additional tumor models to test the anti-tumor effects of WGP............98

Figure 4.13: Neutrophils are not involved in the anti-tumor effects of WGP...........99 
Figure 4.14: WGP does not reduce tumor burden in CCR2 ${ }^{-/-}$mice....................100

Figure 4.15: CCR2+ and CCR2- admix tumor model........................... 100

Figure 4.16: CyTOF of admix CCR2+ and CCR2- admix KPC tumors...............101

Figure 4.17: Bone marrow chimera model to assess the role of central trained immunity in orthotopic pancreatic tumors.............................. 101

Figure 4.18: WGP synergizes with anti-PD-L1 immunotherapy to

Prolong survival................................................................. 102

Figure 4.19: WGP used in the adjuvant setting ............................... 103

Figure 4.20: WGP combined with anti-PD-L1 immunotherapy in th adjuvant setting...103

\section{SUMMARY}

Figure S1: Graphical Abstract.............................................110

Figure S2: Schematic of the use of WGP particles loaded with anti-tumor agents to target the pancreas............................................... 114

\section{METHODS}

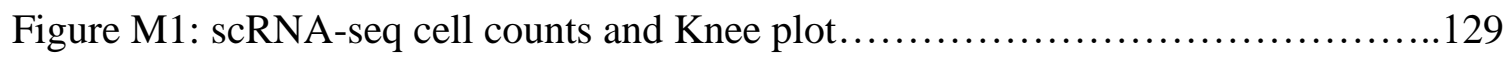




\section{INTRODUCTION}

The diagnosis of pancreatic ductal adenocarcinoma (PDAC) is a devastating one, with only $10 \%$ of patients surviving past 5 years [1]. Although the survival rate since 2014 has increased from $6 \%$ to $10 \%$, pancreatic cancer remains refractory to the majority of currently available therapeutics. In addition, as the demographics of the United States shift, it is projected that pancreatic cancer will become the second leading cause of cancer-related mortality by 2030 and thus presents a significant future challenge for clinicians [2]. Pancreatic cancer is particularly lethal due to the fact that in early stages there are seldom clinical symptoms, which results in $75-80 \%$ of patients being diagnosed with advanced, non-resectable disease $[3,4]$. Even in patients who are eligible for resection, the 5 year survival rate is only 20-25\% [4]. Furthermore, pancreatic cancer has shown little responsiveness to immunotherapies which have shown remarkable effects in other solid tumors [5-8]. The Phase I and II clinical trials using CTLA-4 and PD-1 inhibitors both alone and in combination have been deemed ineffective for the treatment of PDAC, which is likely explained by the non-immunogenic nature of PDAC [9-11].

A major challenge to the successful application of immunotherapy in PDAC is overcoming the immunosuppressive pancreatic tumor microenvironment (TME). PDAC is characterized by a dense pro-tumorigenic desmoplastic stroma, an abundance of immunosuppressive cell subsets within this stroma such as tumor-associated macrophages (TAMs), regulatory T-cells (T-regs), and myeloid-derived suppressor cells (MDSCs), a dearth of activated anti-tumor immune cells, and hypo-vascularity that lends to a hypoxic 
microenvironment [12-14]. Together these conditions make it exceptionally challenging to effectively deliver immunotherapies to the pancreas and for these therapeutics to successfully activate anti-tumor immune responses if they do arrive there. Therefore, novel therapeutics are needed that can not only specifically target the pancreas, but that can also infiltrate the dense desmoplastic stroma and that are capable of inciting robust anti-tumor immune responses despite the immunosuppressive TME.

$\beta$-glucans are a group of biologically active polymers of glucose that are found in the cell wall of bacteria, fungi, edible mushrooms, yeast, barley, oats, wheat and rye $[15,16]$. Though $\beta$-glucan can come in many forms, only molecules with a $\beta(1,3)$-linked D-glucose backbone can act as a biological response modifier (BRM) [17]. Yeast-derived $\beta$-glucan is one such example of a BRM, and is made of a linear backbone of $\beta(1,3)$-linked D-glucose molecules with $\beta(1,6)$ side chains of variable length [18]. This form of $\beta$-glucan has been extensively studied for its ability to reprogram immunosuppressive M2 macrophages into activated, anti-tumor M1 macrophages [19]. More recently however, $\beta$-glucan has also been identified as an inducer of a program of innate immune memory, termed trained innate immunity [20]. Trained immunity is the concept that when innate immune cells are exposed to specific initial stimuli, such as $\beta$-glucan or the Bacillus Calmette-Guérin (BCG) vaccine, they will undergo metabolic, epigenetic and transcriptomic reprogramming [21-23]. Due to this reprogramming, when these cells then come into contact with a secondary, heterologous stimulus, the cell will exhibit enhanced reactivity by means of increased cytokine production $[24,25]$.

In this study, we characterize a novel trafficking mechanism of yeast-derived particulate $\beta$-glucan which when injected IP traffics in large proportion to the pancreas. 
This results in an influx of trained innate immune cells with an activated phenotype into the pancreas that notably do not cause pancreatic destruction or signs of pancreatitis. We show that these trained innate immune cells have antitumor effects, which results in significant tumor control with prolonged survival in orthotopic pancreatic tumor models. Given the failure of the majority of immunotherapies alone and in combination to treat PDAC and the difficulty of targeting these therapeutics to the pancreas, these findings provide a potential breakthrough in not only targeting the pancreas with an immunotherapy, but also in recruiting anti-tumor, pro-inflammatory immune cells to the PDAC TME.

\section{Myeloid Cells in the Tumor Microenvironment}

There are two arms of the immune system: the innate and the adaptive. Of the two, the innate immune system is the most evolutionarily conserved arm, and generates rapid, nonspecific inflammatory responses due to signaling by Pattern Recognition Receptors (PRRs) such as Toll Like Receptors (TLRs). Pathogen Associated Molecular Patterns (PAMPs), which are conserved molecular motifs of pathogens such as bacteria, viruses, and fungi can bind to PRRs which will induce innate immune cell activation [26]. Prototypical examples of PAMPs include bacterial lipopolysaccharides (LPSs) and endotoxins expressed on the cell membrane of gram-negative bacteria. Engagement of PRRs by PAMPs initiates intracellular signaling cascades which result in the production of diverse pro-inflammatory molecules. This response not only targets the invading pathogen, but also is requisite for the activation and recruitment of adaptive immune responses [27]. The innate immune system includes macrophages, Dendritic Cells (DCs), Natural Killer (NK) cells, mast cells, neutrophils, eosinophils, basophils, innate lymphoid cells (ILCs) and proteins of the 
complement cascade. Importantly, while the innate immune system may have evolutionarily evolved to detect foreign invaders such as bacteria and fungi, these pathways also participate in the detection of tumor cells [28].

Adaptive immune responses often steal the spotlight with regards to anti-tumor immunity, and new therapies that promote antitumor immunity have focused primarily on enhancing adaptive immune responses. For example immune checkpoint inhibitors such as anti-Programmed cell Death 1(PD-1) monoclonal antibodies (mAbs) which focus on activating T-cells in the tumor microenvironment (TME) and chimeric antigen receptor (CAR) T-cell infusions have been two of the most studied recent innovations in cancer research [29-31]. Despite this focus on adaptive immune responses, it is important to remember that the activation status of T-cells is not autonomous, but instead closely controlled by innate immune cells within the TME. The importance of T-cells in antitumor immunity have been demonstrated by a positive correlation between tumor infiltrating $\mathrm{T}$ cells and prognosis in several cancer types [32-34]. However, in order to generate antigenspecific T-cells and to initiate T-cell responses, Antigen Presenting Cells (APCs) such as DCs and macrophages are required. In this way, though adaptive cells may exert significant anti-tumor functions, these effects are dependent on the control and signaling of innate immune cells. Though there are many innate immune cell types that play an important role in the pathogenesis of tumors, here macrophages and monocytes will be discussed in detail as these are the innate immune cells that were investigated in relation to trained immunity in this project.

In the context of tumors, three important macrophage/monocyte derivates are tumor associated macrophages (TAMs), myeloid-derived suppressor cells (MDSCs), and resident 
macrophages [35]. TAMs and MDSCs differentiate from immature peripheral blood monocytes which originate from common myeloid progenitors (CMPs) in the bone marrow. In the case of MDSCs, the TME will often secrete growth factors, chemokines and cytokines that promote the accumulation of immature myeloid cells that upon arrival to the TME fail to properly differentiate and proliferate [36]. Circulating monocytes can also migrate to different tissues and are capable of replenishing the populations of resident tissue-specific macrophages such as Kupffer cells in the liver and alveolar macrophages in the lung [37]. Additionally, there are unique subsets of resident macrophages, such as glial cells in the brain, that differentiate from the yolk-sac of a developing embryo and which are maintained through local proliferation [38]. Within the tumor microenvironment, macrophages of all origins take on a wide array of phenotypes according to factors within the TME. Often however, macrophages in the TME take on pro-tumorigenic roles by promoting angiogenesis, immunosuppression, tumor cell invasion and metastasis [39].

TAMs are often the most abundant population of tumor infiltrating immune cells within the TME, and have been associated with a more malignant phenotype and a poor prognosis in various tumor subtypes [40-44]. Macrophages are typically thought to exist in two polarized states, classically activated (M1) and alternatively activated (M2), though realistically the status of macrophages is likely not binary and exists on a sliding spectrum between the two activation states. TAMs often exhibit an alternatively activated, M2, phenotype where they not only produce anti-inflammatory cytokines such as IL-10, IL-13, and TGF- $\beta$, but also can secrete growth factors such as vascular epithelial growth factor (VEGF) and adrenomedullin to directly support angiogenesis [35, 45-47]. These immunosuppressive chemokines, cytokines, and enzymes culminate in the direct inhibition 
of CD8+ and CD4+ effector functions and stimulate the recruitment of regulatory $\mathrm{T}$ cells (Tregs) which further promotes tumor growth [48]. Through the production of matrix metalloproteinases, serine proteases, and cathepsins, TAMs also modify cell-cell junction and disrupt the basal membrane which results in increased capability of tumor cells to invade the primary tumor site and also migrate to distant places to form metastases [4951]. Finally, TAMs are also involved in resistance to chemotherapy and radiotherapy treatment, which are two of the most heavily utilized treatment modalities in cancer [52$55]$.

MDSCs are a heterogenous group of immature myeloid cells that are not typically found in healthy individuals and which are divided into two groups: polymorphonuclear (PMN-MDSCs) and monocytic (M-MDSC). M-MDSCs are mononuclear and express high levels of Ly6C, a prototypical marker for monocytes, and have very low expression of Ly6G, a prototypical marker of neutrophils. PMN-MDSCs on the other hand have a multilobular nucleus, similar to a neutrophil, and have high expression of Ly6G and low expression of Ly6C [56]. In chronic inflammatory states such as cancer, pro-inflammatory cytokines such as PGE2, M-CSF, G-CSF, GM-CSF, TNF- $\alpha$, CXCL12, CCL2 and TGF- $\beta$ are released locally by immune cells, stromal cells and tumor cells, which can impact myelopoiesis in the bone marrow and skew the differentiation of immature myeloid cells recruited to the TME and halt their ability to differentiate [57-60]. These immature myeloid cells take on potent anti-inflammatory phenotypes once they arrive at the TME and are known to significantly impair CD4+ and CD8+ T-cell functions, induce NK cell dysfunction, prevent DC maturation and induce Tregs [61-66]. Due to these varied immunosuppressive functions, the presence and number of MDSCs in tumors have been 
associated with a shorter overall survival and shorter progression-free survival after treatment [67-69].

Splenic red-pulp macrophages, lung alveolar macrophages, brain microglia, epidermal Langerhans cells, large peritoneal macrophages, $\mathrm{F} 4 / 80^{\mathrm{hi}}$ pancreatic macrophages, and cardiac macrophages are all examples of tissue-resident macrophages that are known to develop in the yolk sac of embryos independently of hematopoietic stem cells (HSCs) and persist in adult tissues [70-73]. Many of these tissue-resident macrophage populations are maintained through local proliferation within the tissue, though BMderived circulating monocytes can also migrate to tissues and contribute to the tissue resident pool of macrophages [74-76]. Like TAMs recruited from the periphery, resident macrophages present within the TME also play a role in tumor pathogenesis, though their function has not been as well defined as other macrophage populations. For example, tissue-resident interstitial macrophages in the lungs of mice were found to significantly contribute to the pool of tumor-associated macrophages along with the CCR2-dependent recruited macrophages. Additionally, these resident macrophages were found to associate with increased tumor growth [77]. Resident macrophages found within the omentum have been found to be involved in the metastatic spread of ovarian cancer, and in models of PDAC, tissue resident embryonic derived macrophages were also shown to promote fibrosis and tumor progression $[78,79]$. Even in the brain, resident microglia can exhibit anti-tumor functions, however they can also be polarized into immunosuppressive tumorsupporting cells by specific tumor-derived soluble factors which cause them to contribute to tumor angiogenesis, metastasis, dormancy and relapse [80-83]. It has been observed that resident macrophages pathogenically contribute to the creation of a premetastatic niche 
in organs that are targets of metastasis formation. For example, in a model of metastatic pancreatic cancer, it was found that liver-resident macrophages, Kupffer cells, uptake cancer-derived exosomes released from the primary tumor which causes them to secrete TGF- $\beta$ which activates hepatic stellate cells to transform into myofibroblasts that produce fibronectin and recruit immune cells to the liver [84, 85]. Accordingly, it is important to distinguish between infiltrating macrophages/ circulating monocyte-derived macrophages and resident macrophages in order to fully understand the role of TAMs and to develop therapeutics that can effectively target pro-tumorigenic populations.

\section{The Tumor Microenvironment of Pancreatic Cancer}

PDAC accounts for more than $90 \%$ of all pancreatic cancer cases, and is also considered to be one of the most aggressive forms of cancer [86]. The long-term clinical outcomes of pancreatic cancer are dismal, with the median survival from time of diagnosis being 3-6 months in patients who do not receive treatment, and 23 months in patients who receive resectional surgery and adjuvant therapy [87]. One reason that pancreatic cancer is so deadly is that it is often diagnosed at a late stage of cancer progression, as patients often only begin showing non-specific symptoms of the malignancy once it has grown large enough to press on the bile duct, resulting in symptoms of jaundice, weight loss and anorexia. Pancreatic cancer has a propensity for vascular invasion, rapid and robust lymphatic spread, and perineural invasion, meaning that tumor cells surround nerves which allows for an alternative route for metastatic spread and results in considerable pain [88]. To date, most oncological interventions have failed to produce benefits in long-term survival and resection remains one of the most effective modalities. Unfortunately, a mere 
$10-20 \%$ of patients with primary tumors in the head of the pancreas, and less than $3 \%$ of patients with a primary tumor in the head or tail of the pancreas are candidates for resection [89-91]. The primary goal of surgical resection is to achieve a negative resection margin (R0), however despite this, most patients will develop a recurrence at some point following surgical resection [92]. Most patients diagnosed with PDAC will go on to receive some form of adjuvant chemotherapy. FOLFIRINOX, which is a chemotherapy regimen consisting of Leucovorin, 5-fluotouracil, irinotecan and oxaliplatin, is currently the standard adjuvant therapy used and has led to relatively improved outcomes for patients with PDAC, however are not sufficient for patients with advanced disease [93, 94]. Another particular challenge of PDAC is the high degree of genetic heterogeneity that occurs not only between patients but within a single primary tumor [86]. Whereas in lung cancer or melanoma where specific genetic mutations, such as EGFR and BRAF, respectively, provide excellent targets for interventional therapies, within a single PDAC tumor there exist a plethora of mutations that are not shared between patients [95]. Additionally, the use of immunotherapy, such as immune checkpoint mAbs anti-CTLA-4, anti-PD-1 and anti-PD-L1 have not shown meaningful clinical efficacy and are currently not approved for use in patients with pancreatic cancer[6, 96].

An important reason that PDAC shows such resistance to commonly used adjuvant therapies such as chemotherapy and immunotherapy is the immunosuppressive TME. One hallmark of the PDAC TME that is present in both the primary tumor and sites of distant metastases is the formation of a dense desmoplastic stroma around the tumor [97]. Pancreatic stellate cells are activated by tumor-secreted factors which causes these myofibroblast-like cells to initiate pro-fibrotic mechanisms. This fibrotic desmoplastic 
layer around the tumor creates a physical barrier around tumors that limits immune cell invasion, promotes tumor angiogenesis and prevents the delivery of systemically delivered therapeutics such as chemotherapy and immunotherapy from arriving at the tumor site [98, 99]. Importantly, PDAC has also been classified as an immunologically "cold" tumor meaning that there are few activated anti-tumor T-cells and many immunosuppressive Tregs, TAMs and MDSCs. In PDAC, an increased number of Tregs, MDSCs and TAMs have all has been associated with promoting PDAC and decreased overall survival [98, 100-102].

The dense desmoplastic stroma that decreases the formation of normal vasculature also creates a hypoxic environment that effectively recruits immunosuppressive MDSCs and assists in their differentiation upon arrival to the tumor site [103]. Patients with PDAC exhibit elevated levels of systemic and intra-tumoral MDSCs upon progression of disease, and within the TME MDSCs are known to directly suppress the formation of antigenspecific T-cells and induce Tregs [104-106]. Upon migration to the tumor site, MDSCs have also been shown to differentiate into TAMs, though in murine models of pancreatic cancer, $50 \%$ of TAMs were also shown to derive from resident macrophage populations [78, 107]. Accordingly, analysis of patient's PDAC samples show that TAMs exhibit M2 markers such as CD206 and CD163 and secrete high levels of IL-10. An increased number of M2 macrophages is associated with larger tumor size, early recurrence in the liver, local recurrence and shorted survival in patients [108]. TAMs have been specifically shown to mediate gemcitabine resistance in PDAC by upregulating cytidine deaminase (CDA) which is the enzyme responsible for metabolizing gemcitabine after its transport into the cell, thereby expediting its inactivation [109]. TAMs also have been shown to suppress 
clinical responses to irradiation and angiogenic inhibitors that are commonly used in pancreatic cancer [110].

Due to the pronounced presence of MDSCs and TAMs within the PDAC TME combined with their ability to modulate the function of many other immune cell types, myeloid cells within the TME have become the popular target for new anti-cancer therapeutics. Accordingly, it has been shown that depleting tumor-infiltrating macrophages can improve chemotherapeutic responses and can relieve immunosuppression [111]. Further, while it has been shown that checkpoint inhibitor therapies such as anti-CTLA-4 or anti-PD-L1 as a monotherapy are not effective in pancreatic cancer, the depletion of TAMs using CSF-1 inhibition synergized with checkpoint immunotherapy in PDAC [112]. This provides a convincing rationale to develop therapeutics with the goal of reprograming immunosuppressive TAMs and MDSCs within the TME in order to unleash the potential of immunotherapy in the setting of PDAC.

\section{Trained Innate Immunity}

Given the critical role that innate immune cells play in the TME of PDAC, we now turn our attention to a program of innate immune cell memory, called trained immunity, which we propose has the potential to reprogram immunosuppressive myeloid cells within the TME.

Throughout evolution, the vast majority of cellular life has existed without the protection from adaptive immune responses. Adaptive immunity is actually a relatively 
contemporary evolutionary adaptation, where until the emergence of jawed fish 500 million years ago, multicellular organisms existed only with features of innate immunity $[113,114]$. In the absence of protective memory features of adaptive immunity, plants, protists, invertebrates and lower animals evolved ways to protect themselves from recurrent infections through the development of systemic acquired resistance (SAR). SAR is a program of non-specific protection that is responsible for the observation that following inoculation with attenuated organisms, plants are protected from subsequent infections from several infectious agents such as fungi, viruses and bacterial pathogens [20, 115]. Though ancient, the ability to mount memory-like innate immune responses certainly conferred an evolutionary advantage, and this capability was thus retained as evolution progressed towards higher vertebrates. These mechanisms underpin the recent discovery of trained innate immunity, which posits that mammalian innate immune cells exhibit immunological memory of previous pathogen interactions which allows them to better respond to and defend against future infections, even from different sources.

The concept of trained immunity in humans has been present for almost a century. In 1931, a Swedish study showed that infants given the Bacille Calmette-Guérin (BCG) vaccine against Mycobacterium tuberculosis (TB) exhibited increased survival rates as compared to infants that did not receive the BCG vaccine, which could not be only due to having immunity to TB [116]. Accordingly, epidemiologists were the first to truly characterize trained immunity, where it was noted that recipients of the smallpox vaccine were not only protected against smallpox but also other infections such as measles, scarlet fever and syphilis [117]. In the late 1900s, a number of additional studies came out which further characterized trained immunity, where it was shown that exposure to glucans, fungi 
and other vaccines, such as the measles vaccine, afforded non-specific protection from later heterologous infections [118-122].

A 2012 study by Quintin et al, more formally confirmed the phenomenon of innate immune memory by showing that mice exposed to a low dose of C. albicans induced protection against reinfection with C. albicans in Rag-1 deficient mice which lack B and T-cells. Further, they showed that the protection to C. albicans was driven by macrophages and monocytes, which produced increased levels of TNF- $\alpha$, IL- 6 , and IL-1 $\beta$ and showed an increased phagocytic capacity [123]. Importantly, they also showed that a specific component within the cell wall of C. albicans, $\beta$-1,3-(D)-glucan ( $\beta$-glucan), was responsible for initiating these effects. Since then, a great deal of investigation has been performed to understand the mechanisms of trained immunity involving $\beta$-glucan. Human and animal studies alike have shown that exposure to $\beta$-glucan protects from secondary infections such as Staphylococcus aureus, TB, Leishmania braziliensis and influenza [124-126]. Together, these studies support that $\beta$-glucan is a potent inducer of trained immunity such that when innate immune cells that have been exposed to $\beta$-glucan are exposed to heterologous secondary stimuli of many different etiologies, they will mount a qualitatively different, and quantitatively stronger response than cells that are untrained 
[127]. In experiments involving trained immunity, LPS is often used as a secondary stimulus, and it is capable of clearly re-stimulating trained cells (Figure I.1).

\section{Figure I.1: Schematic of trained immunity}

Cells are exposed to an initial training stimulus such as $\beta$-glucan after which they undergo epigenetic, metabolic and mitochondrial reprogramming. When cells are then exposed to

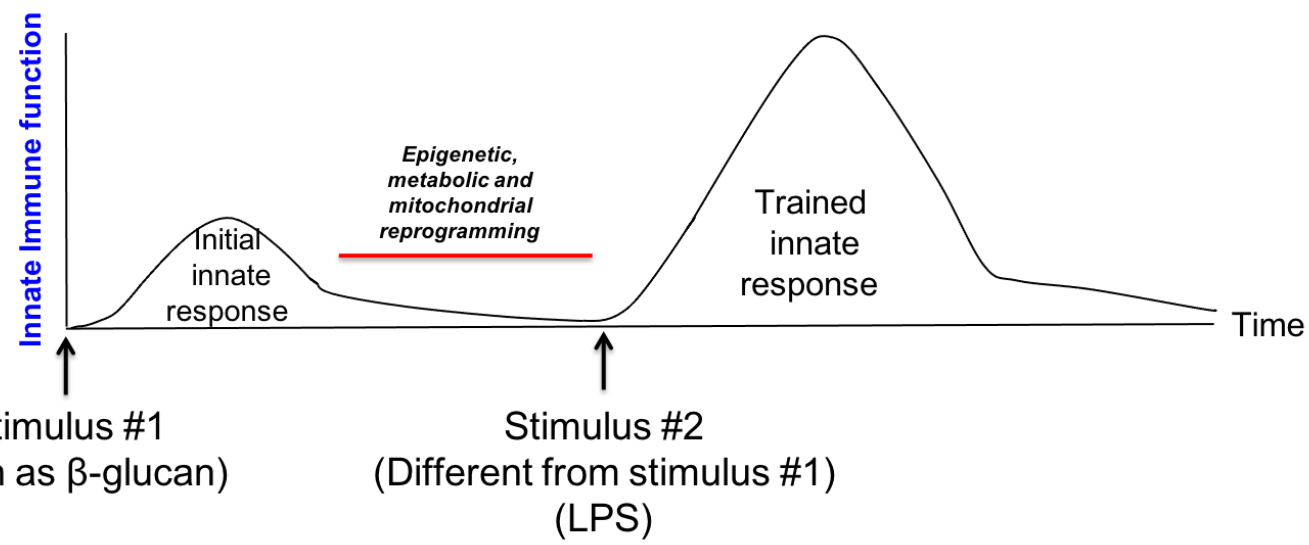

a secondary stimulus, such as LPS, cells then exhibited responses that are more robust than the initial stimulus or the secondary stimulus alone. In effect, the initial stimulus causes cells to have a more vigorous response to a secondary stimulus.

Trained innate immunity primarily involves the training of macrophages and monocytes, though DCs, Natural Killer (NK) cells and neutrophils have been shown to be involved in trained immunity [128-130]. Following induction, it has been observed that trained immunity can last from weeks to months. Given that monocytes in the periphery are typically short lived, this brought into question whether cells in the periphery are directly trained or whether training agents may impact the BM thus leading to more lasting effects. Additionally, because cells in the periphery are often terminally differentiated and therefore unable to give rise to a cohort of trained progeny, it was hypothesized that trained 
immunity may directly impact the HSCs in the BM which then gives rise to trained cells systemically. Fittingly, mice injected with intraperitoneal (IP) $\beta$-glucan show a biased expansion of $\mathrm{Lin}^{-} \mathrm{Sca}^{+}{ }^{+} \mathrm{CKit}^{+}$(LSKs) and Multipotent Myeloid Progenitors (MMPs) in the BM [131]. Under the current paradigm of trained immunity, the education of HSCs and the induction of myelopoiesis in the BM is responsible for the generation of "central" memory which then creates a repertoire of innate cells possessing innate immune memory features, which then migrate to the peripheral tissues to generate peripheral memory [132, $133]$.

In myeloid cells, loci encoding inflammatory signaling are often in a repressed configuration, which is due to low levels of acetylation of histones and minimal RNA polymerase II present on enhancer and promoter regions [134, 135]. Following exposure to inducers of trained immunity, drastic changes to the accessibility of DNA take place through the acetylation of histones and the recruitment of RNA polymerase II, and result in the activation and upregulation of gene expression that can be on the order of 100 times higher than baselines levels. These changes are also driven by stimulation-responsive transcription factors such as NF-kB and STAT proteins being recruited to gene promoters, which then recruit co-activators such as histone acetyltransferases and chromatin remodelers. Together, these significantly increase the accessibility of chromatin, which allows for better accessibility of proteins involved in transcription, and ultimately more robust responses upon restimulation [127, 136-138]. While inducers of trained immunity generally work by increasing the activation status of innate immune cells through these described epigenetic modifications, the exact mechanisms can be different among initial stimuli [139]. 
Dectin- 1 is the main receptor for $\beta$-glucan, and accordingly it was shown that invitro models of trained immunity using $\beta$-glucan are mediated by dectin-1 signaling [140]. Stimulation of myeloid cells with $\beta$-glucan has been shown to directly recruit and activate SyK kinase through dectin-1 followed by recruitment of Card9 to form the Card9/Bcl10/Malt-1 complex that activates the IкB kinase complex resulting in the activation of key transcription factor NF- $\mathrm{KB}$. Activation of NF- $\mathrm{\kappa B}$ is then able to initiate a pro-inflammatory signaling cascade [141-143]. While the specific molecular mechanisms of trained immunity are still being investigated, mounting evidence shows that following dectin-1 activation, transcriptomic, epigenetic, metabolic, and mitochondrial alterations take place which each plays an integral role in the initiation and expression of trained immunity.

Activation of the Dectin-1 receptor by $\beta$-glucan has been shown to directly cause important changes to the epigenetic status of immune gene promoters. An example of the epigenetic priming induced by $\beta$-glucan is that upon Dectin- 1 activation, nuclear factor of activated T-cells (NFAT-1) is dephosphorylated, which results in its translocation through the nuclear membrane. NFAT-1 mediates $\beta$-glucan-driven epigenetic training by upregulating immune gene-priming long non-coding RNAs (IP-incRNAs) which culminates in increased levels of trimethylation of histone $\mathrm{H} 3$ at lysine 4 (H3K4me3) at promoter sites [123, 144]. High levels of H3K4me3 are associated with robust levels of gene expression, and so this epigenetic effect results in more vigorous cytokine production upon re-stimulation of $\beta$-glucan-primed immune cells [145]. Such epigenetic modifications driven by $\beta$-glucan result in inflammatory genes that are ideally positioned to be rapidly activated by secondary infections or stimuli, such as a virus, bacteria or even a tumor cell. 
This explains why myeloid cells trained with $\beta$-glucan release increased levels of TNF- $\alpha$, IL-6, and IL-1 $\beta$ following restimulation with LPS.

Metabolic changes are also a prominent feature of $\beta$-glucan induced trained immunity, as vital energy metabolites regulate chromatin-modifying epigenetic enzymes, methylation, histone modification, and the position of the nucleosome by acting as substrates and co-factors. Consequently, the energy state of a cell and the metabolic programs that are initiated as a result of $\beta$-glucan stimulation tightly modulate the transcription of immunogenic genes [146]. The metabolic switch from oxidative phosphorylation toward aerobic glycolysis is a key feature of trained immunity, which has been shown to be mediated through the AKT/mTOR/HIF1 $\alpha$ pathway [21]. Other notable metabolic features of trained immunity are a decrease in itaconate, a product of the decarboxylation of cis-aconitate, and increased fumarate and mevalonate accumulation through upregulation of the TCA cycle following stimulation with LPS. $\beta$-glucan signaling notably inhibits the LPS mediated upregulation of immune-responsive gene-1 (IRG-1), the enzyme that is responsible for itaconate generation, and stimulates the activity of succinate dehydrogenase, leading to increased fumarate production [147]. This is critically important as itaconate is known to induce immune tolerance and anti-inflammatory properties in human monocytes.

To date, mechanisms of trained immunity have been primarily studied with regards to infection. Recently however, two studies have highlighted that it may also be possible to utilize the induction of trained immunity to kill cancer cells. One of these studies by Kalafati et al., describes that pre-treatment of mice with $\beta$-glucan induces long-term transcriptomic and epigenetic rewiring of granulopoiesis which results in the creation of 
neutrophils that have an anti-tumor phenotype [130]. In the same issue of Cell, another paper regarding the use of trained immunity in the setting of cancer appeared, though in this paper the authors utilized a novel nano-biologic therapeutic called MTP-HDL. Following injection, MTP-HDL was found to target and accumulate in the hematopoietic organs of mice and non-human primates. In the BM specifically, MTP-HDL was shown to initiate epigenetic modifications in MPPs that are consistent with the initiation of trained immunity. For example, they showed that MPPs had higher chromatin accessibility near the promoters of genes that regulate TNF- $\alpha$ and IL-6. During myelopoiesis, these trained myeloid cells were able to initiate anti-tumor effects in a B16F10 subcutaneous model of melanoma and not only showed inhibition of tumor growth, but to also showed the capability to potentiate the function of immune checkpoint inhibitor therapy [148, 149].

These two recent articles are the first descriptions of the use of $\beta$-glucan-induced trained immunity in the setting of cancer, however both studies utilized subcutaneous models of tumors, which do not fully encapsulate the organ-specific features of immunosuppression that are present in tumors. Studies over the last three decades have consistently shown that the use of subcutaneous tumor models rarely result in the occurrence of metastatic disease, and they never result in metastatic disease in the clinically relevant organs according to the type of tumor cell used [150-152]. For example pancreatic cancer often metastasizes to the liver and lung, however a subcutaneous model of pancreatic cancer would not result in metastases to these organs. Additionally, the recruitment of anti-tumor immune cells is highly dependent on the vasculature both outside and within a tumor, which are controlled by angiogenic processes at the tumor site. There is evidence that tumor vascularity is organ-site specific and therefore subcutaneous models 
of cancer fail to fully recapitulate the true vascular environment of a tumor [153]. Additionally, in the setting of pancreatic cancer, for example, pancreatic stellate and stromal cells play a critical role in tumor progression, as do resident macrophages Kupffer cells in the setting of hepatocellular carcinoma. Subcutaneous models fail to take into account these organ-specific drivers of immune-evasion, which can lead to overly extrapolated or interpreted conclusions about the efficacy of anti-tumor therapeutics [154]. Overall, the use of subcutaneous models of cancer leads to shortcomings in the understanding of blood flow, intravascular-tumor cell trafficking, extravasation of tumor cells, the architecture of the TME, and the distribution and targeting efficacy of cellular and chemical therapeutics $[155,156]$. As such, while these initial studies that investigate the role of trained immunity in combating cancer are exciting, studies such as the ones presented in this dissertation are critical to understanding if the anti-tumor mechanisms of trained immunity are applicable in in situ pre-clinical models of cancer. 


\section{CHAPTER I}

\section{THE TRAFFICKING AND BIODISTRIBUTION OF $\beta$-GLUCAN}

\section{CHAPTER I INTRODUCTION}

Even before $\beta$-glucan was identified to be involved in initiating trained innate immune responses, $\beta$-glucan has been extensively studied for its immunomodulatory properties. Understanding the trafficking mechanisms of $\beta$-glucan has thus been a focus of $\beta$-glucan related research. Of all of the routes of administration, orally administered $\beta$-glucan has been the most extensively studied, however intravenous (IV) and intraperitoneal (IP) injections of $\beta$-glucan have also been used. Following oral administration, $\beta$-glucans move into the proximal small intestine where they are phagocytosed by intestinal epithelial cells or pinocytic microfold cells (M-cells) which transport $\beta$-glucan from the intestinal lumen to immune cells within Peyer's patches [157-159]. Following exposure to $\beta$-glucan, gastrointestinal macrophages then migrate through the bloodstream toward the lymph system, ultimately resulting in trafficking to the bone marrow, spleen and lymph nodes [160].

IP injection of $\beta$-glucan has been less extensively studied than oral administration, though there are some studies which show that IP injection has more profound effects on immunological activity than does oral administration [161]. Importantly, experiments utilizing $\beta$-glucan for the initiation of trained immunity typically use a model of 
intraperitoneal injection. Zheng et al. found that following IP administration, $\beta$ glucan was ingested by resident peritoneal macrophages which then trafficked to the lymph nodes, thymus and the tumor site in BALB/c mice bearing subcutaneous $\mathrm{H} 22$ hepatocellular carcinomas [162]. Other than this study, there exists sparse detailed information about where exactly $\beta$-glucan traffics to following IP injection. As such, we first sought to identify where $\beta$-glucan traffics to following IP administration. In these studies we utilize a highly purified form of $\beta$-glucan, whole glucan particles (WGP), that was purified from Saccharomyces cerevisiae.

\section{CHAPTER I RESULTS}

To assess the trafficking of particulate $\beta$-glucan, WGP was tagged with (5-(4,6Dichlorotriazinyl) Aminofluorescein) (DTAF), and injected IP into wild-type (WT) C57BL/6 mice. Three days following IP administration (3-day WGP), the lung, bone marrow, spleen, inguinal and mesenteric lymph nodes, peritoneal cavity cells, and pancreas were harvested to detect the presence of DTAF-WGP. While there was some trafficking of the DTAF-WGP to the BM, spleen, and mesenteric lymph nodes, and residual DTAF-WGP in the peritoneal cavity, the pancreas showed a striking and unexpected presence of the DTAF-WGP (Fig 1.1A). In fact, the pancreas appeared bright yellow upon gross visual analysis (Fig 1.1B). To further assess this trafficking and to ensure that the DTAF label was not involved in the trafficking mechanism, WGP was radiolabeled with ${ }^{89} \mathrm{Zr}$ and injected IP (Fig 1.2A) or incubated for 2 hours with peritoneal macrophages that were then injected IP (Fig 1.2B). Mice were first imaged using a PET/CT scan 48 hours following injection and green circles are used to indicate the observed preferential accumulation of 
the ${ }^{89} \mathrm{Zr}$-WGP in the pancreas. A necroscopy was then performed and the radioactive signature of each organ was measured. In accordance with the flow cytometry data, ${ }^{89} \mathrm{Zr}$ WGP trafficked in large quantities to the pancreas, and was found in lower levels in the spleen, liver and intestinal system. Peritoneal macrophages that were cultured with ${ }^{89} \mathrm{Zr}$ WGP and then injected IP had similar though slightly more diversified trafficking than the pure ${ }^{89} \mathrm{Zr}$-WGP, and also accumulated most prominently in the pancreas (Fig 1.2B).

In an effort to assess the role that the known receptor of WGP played in the trafficking, mice lacking the C-type lectin receptor, Dectin-1, (Dectin- $1^{-/-}$mice) were injected with DTAF-WGP and 48 hours later the pancreases were harvested. As compared to WT mice, Dectin- $1^{-/}$mice showed a 5-fold decrease in the amount of WGP that trafficked to the pancreas, as assessed by flow cytometry (Fig 1.3A). ${ }^{89} \mathrm{Zr}$-WGP was also injected IP into WT mice and Dectin- $1^{-/}$mice. As compared to WT animals, there was significantly less trafficking of WGP to the pancreas of Dectin- $-^{-/-}$mice (Fig 1.3B). To ensure that this process was in fact $\beta$-glucan specific, a polystyrene-based latex $3 \mu \mathrm{m}$ fluorescent particle, the same size as a WGP particle, was injected IP and was not found to accumulate in the pancreas (Fig 1.4). Finally, as it had been observed that WGP that had been phagocytosed by peritoneal macrophages also trafficked to the pancreas upon IP injection of the WGPloaded peritoneal macrophages, we also wanted to know whether peritoneal macrophages phagocytosed WGP in vivo. To investigate this, DTAF-WGP was injected into mice IP, and 4 hours later the peritoneal macrophages were harvested. Confocal microscopy was performed to assess if peritoneal macrophages phagocytosed IP injected DTAF-WGP (Fig 1.5). This study showed that peritoneal macrophages did phagocytose WGP in vivo, as the DTAF-WGP could be clearly seen within these cells in the confocal images. 
It appeared form these studies that there was a connection between the peritoneal cavity contents and the pancreas. To further understand how WGP trafficked from the peritoneum to the pancreas, peritoneal macrophages were harvested from WT mice and stained with CFSE. These cells were then adoptively transferred into recipient mice and these mice received either IP PBS or WGP. Mice that received IP WGP displayed more CFSE+ macrophages trafficking to the pancreas than those that received PBS (Figure 1.6). This highlights that there exists a baseline flux of macrophages from the peritoneum to the pancreas that can be utilized and upregulated in the presence of IP WGP. 


\section{CHAPTER I FIGURES}

A
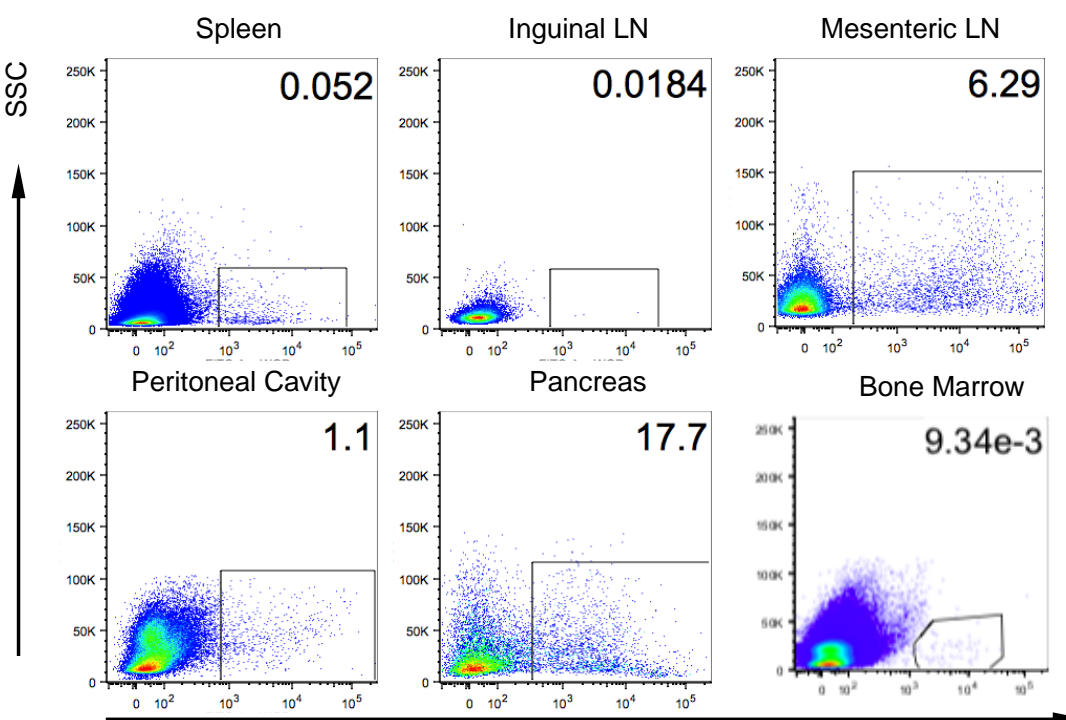

DTAF-WGP

B

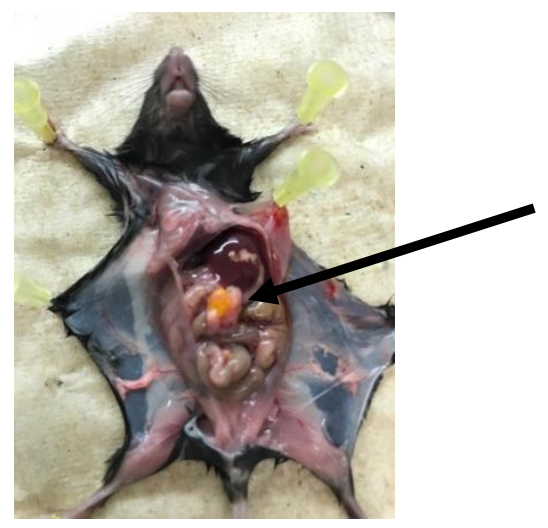

\section{Figure 1.1: IP DTAF WGP preferentially accumulates in the pancreas}

(A) DTAF-WGP was injected I.P. and 3 days later different tissues were harvested and assessed for the presence of the DTAF-WGP by flow cytometry. (B) Gross anatomical view of the presence of DTAF-WGP in the pancreas causing it to appear bright yellow. 
A

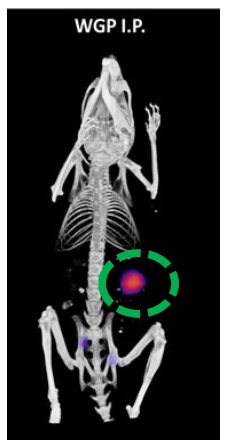

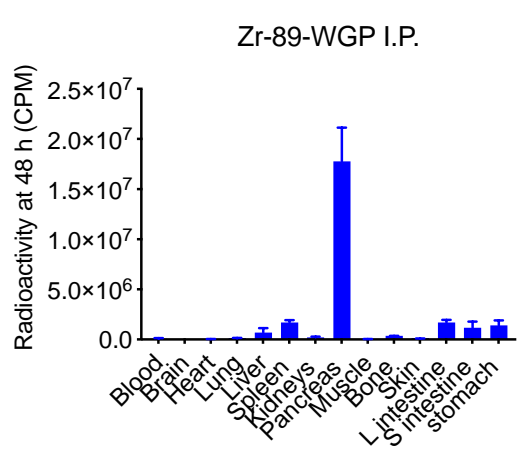

B
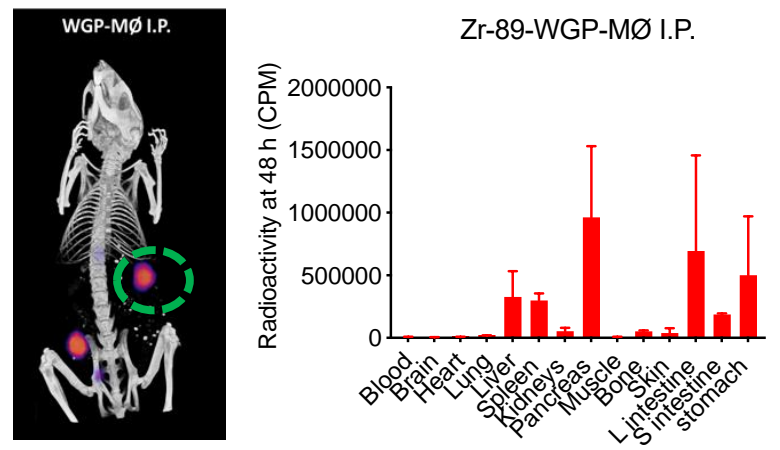

Figure 1.2: Analysis of WGP trafficking using radio-labeled ${ }^{89} \mathrm{Zr}$-WGP

(A) WGP was labeled with ${ }^{89} \mathrm{Zr}$-WGP or (B) peritoneal macrophages were incubated with ${ }^{89} \mathrm{Zr}-\mathrm{WGP}$ and washed, followed by I.P. injection. PET/CT imaging displays the trafficking of the ${ }^{89} \mathrm{Zr}$-WGP throughout the body after 48 hours. Following the PET/CT scan, organs were removed and individually assessed for radioactivity using a gamma counter. $(n=3)$. 
A

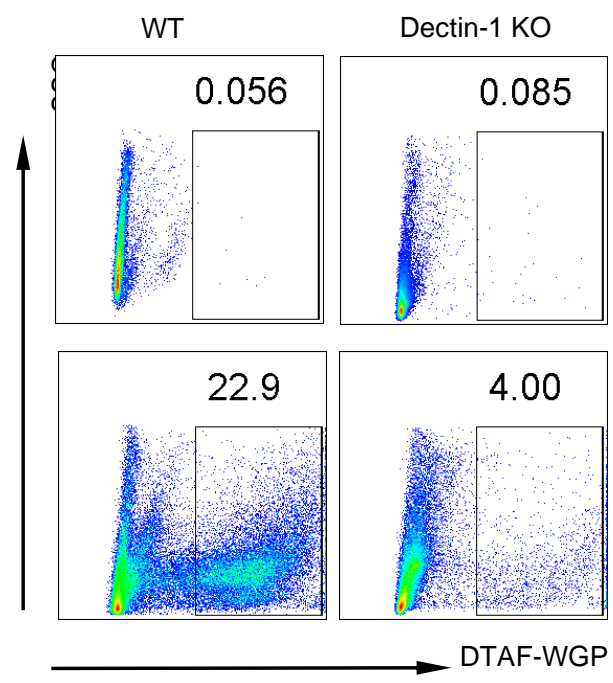

B

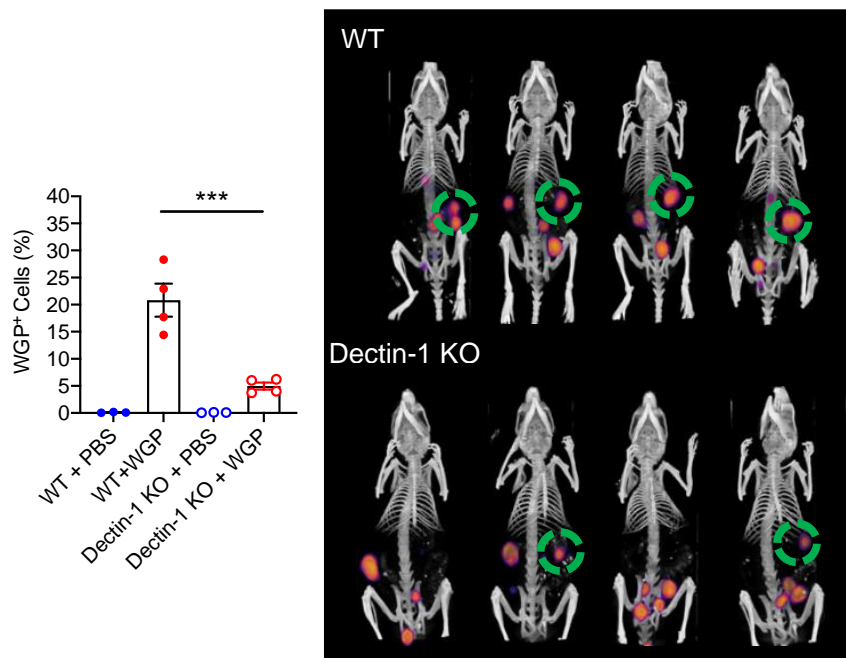

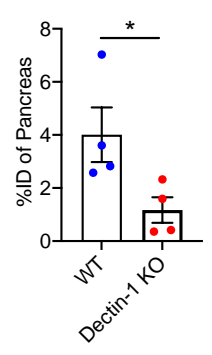

Figure 1.3: Dectin-1 regulates the trafficking of WGP to the pancreas

(A) Dectin- $1^{-/}$mice or WT mice were injected with DTAF-WGP and the accumulation of DTAF-WGP in the pancreas was assessed by flow cytometry. $(n=4)($ B $)$ Dectin- ${ }^{-/-}$mice or WT mice were injected with ${ }^{89} \mathrm{Zr}$-WGP and 48 hours later a PET/CT was used to assess the amount in the pancreas. The signal was quantified by reporting the $\%$ of injected dose (\%ID) in the pancreas. (PBS $n=4$, WGP $n=4)$ Data are represented as mean \pm SEM. $* p$ $<.05, * * * p<.001$ 


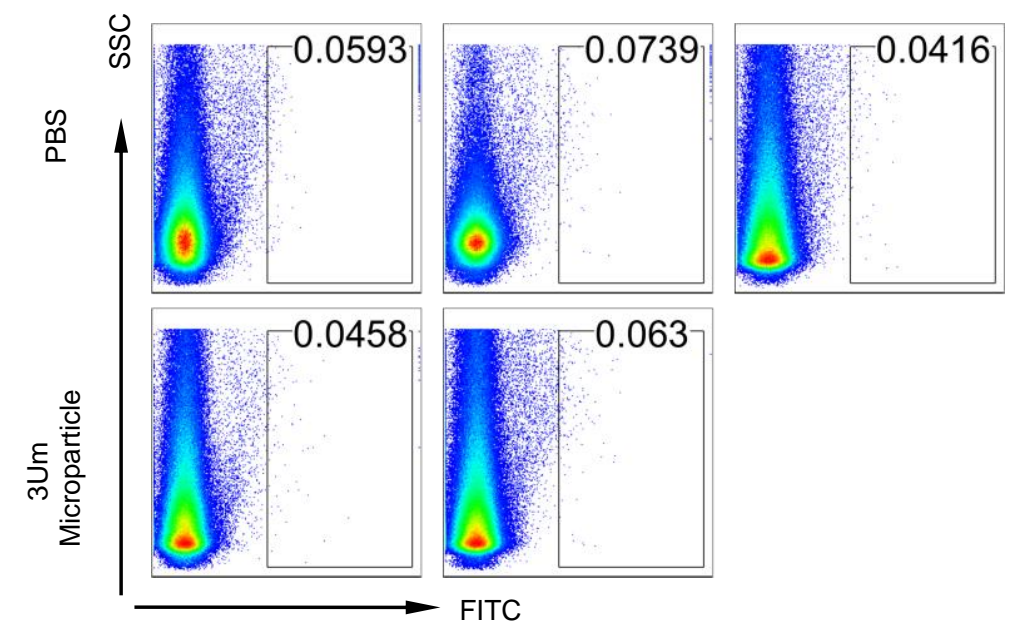

Figure 1.4: A polystyrene bead the same size as WGP does not accumulate in the pancreas.

Fluorescent $3 \mu \mathrm{m}$ polystyrene beads or PBS were injected IP and 24 hours later the pancreas was assessed for the presence of a fluorescent signal, indicating trafficking.

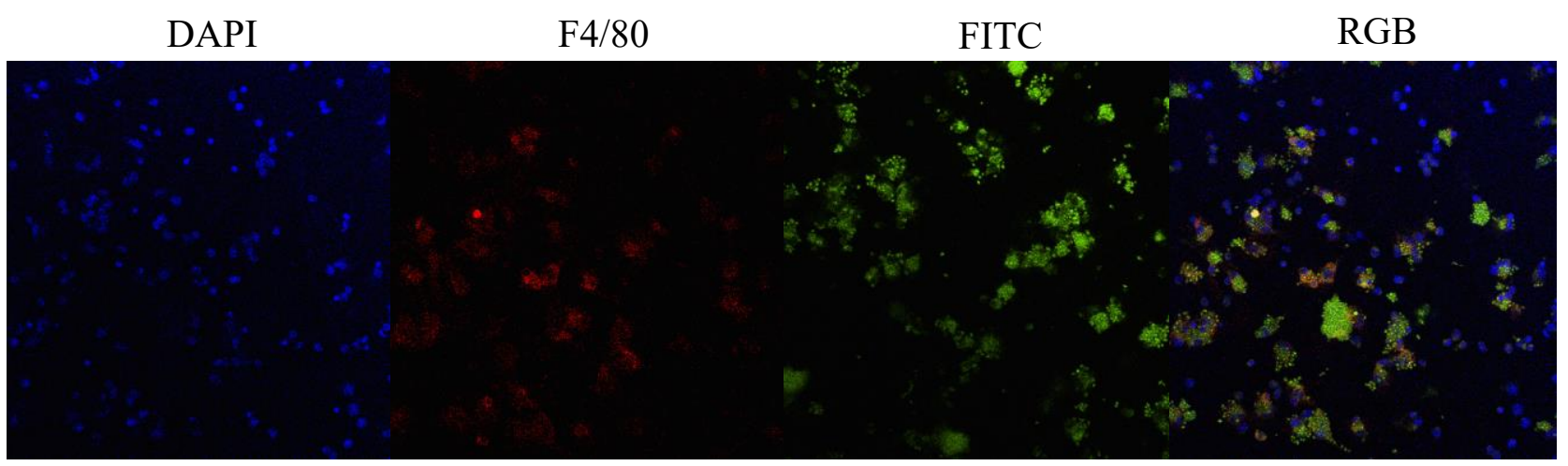

Figure 1.5: Confocal microscopy of in vivo phagocytosis of DTAF-WGP

Mice were injected IP with DTAF-WGP and 4 hours later mice were euthanized, peritoneal macrophages were harvested, cytospun and analyzed using confocal microscopy. 

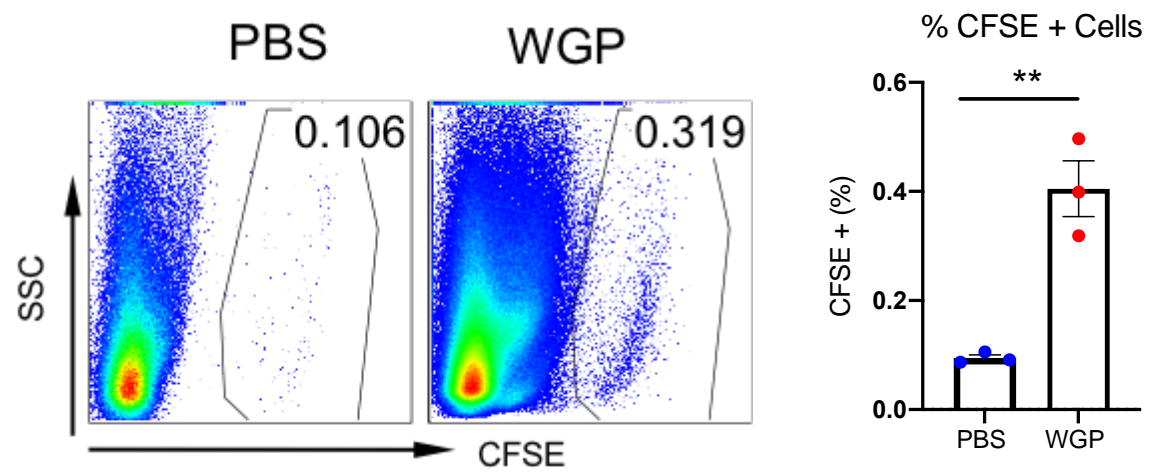

Figure 1.6: There is a transient flux of macrophages between the peritoneal cavity and the pancreas that is utilized to deliver WGP to the pancreas

Peritoneal macrophages were harvested from WT mice and incubated with CFSE. These cells were then adoptively transferred IP into WT mice. 1 day after transfer of CFSE+ peritoneal macrophages, mice were injected with WGP and 24 hours following the pancreata were harvested and the presence of CFSE+ macrophages was measured using flow cytometry. (PBS n=3, WGP $\mathrm{n}=3$ ) Data are represented as mean $\pm \mathrm{SEM}, * * p<.01$ 


\section{CHAPTER I DISCUSSION}

As stated earlier, though the IP administration of $\beta$-glucan has been broadly used for inducing trained immunity, it has not been well established where it traffics to following injection. These studies identify that WGP traffics in small quantities to the lymph system and to the bone marrow. It is this trafficking to the bone marrow that likely causes the effects of central trained immunity. Surprisingly, we found that WGP also trafficked in large proportions to the pancreas which has not been reported before. It was shown that $\beta$ glucan can traffic directly into the pancreas and can also be phagocytosed by macrophages which then traffic into the pancreas. While the relationship between the peritoneal cavity and the pancreas has not been well defined, studies relating to the pathophysiology of acute pancreatitis (AP) have identified that peritoneal macrophages are a principal contributor to the inflammatory response in AP [163]. Our imaging data indeed show that peritoneal macrophages that phagocytose isotope-labeled WGP primarily traffic to the pancreas. Additionally, in an experiment in which CFSE labeled peritoneal macrophages were adoptively transferred IP, these macrophages were found at low concentrations in the pancreas 24 hours later. Studies on liver injury have further supported this model of a dynamic interchange of cells between the peritoneal cavity and solid organs that is independent of the circulation [164]. Together this suggests that even in homeostatic conditions there exists a basal level of immune cell exchange between the pancreas and peritoneal cavity that can be exploited in the setting of particulate $\beta$-glucan.

While we had identified that macrophages that had phagocytosed WGP traffic to the pancreas and carry WGP with it, we also assumed that naked DTAF-WGP may also 
traffic to the pancreas due to the visually yellow appearance of the pancreas. To try and understand whether peritoneal macrophages were critical in the trafficking of WGP to the pancreas, we sought to deplete peritoneal macrophages using liposomal clodronate [165]. Despite reports that IP injection of liposomal clodronate results a clean depletion of peritoneal macrophages, we found that one day following injection, macrophages were not entirely depleted, and further that the immunological makeup of the peritoneal compartment was altered to have increased numbers of monocytes and neutrophils. Further studies are needed to determine if peritoneal macrophages are requisite for WGP trafficking to the pancreas or if naked WGP may traffic there entirely without the assistance of them.

While the impact of $\beta$-glucan on the immune cells of the pancreas will be explored in future sections of this dissertation, this tropism of $\beta$-glucan to the pancreas carries interesting clinical applications given that $\beta$-glucan has the capability to function as a delivery vesicle for cancer therapeutics. Glucan particles are hollow, and as a result have become the subject of research aiming to utilize these properties to employ $\beta$-glucan as a vesicle to encapsulate, transport, deliver and release therapeutics within the TME, and specifically to macrophages. Several groups have already shown that it is possible to load $\beta$-glucan with different electrostatically-bound compounds, such as siRNA, DNA, protein, and small molecules in order to deliver these payloads to macrophages and DCs [166-169]. In the setting of pancreatic cancer, where it is not only exceptionally challenging to target systemic therapies to the pancreas, but it is also imperative to deliver therapeutics that can reprogram the immunosuppressive TME, this poses an attractive treatment strategy. For example, in one study researchers developed a glucan-based siRNA carrier system which 
was shown to effectively assemble siRNA into uniformly distributed nanoparticles. On administration, these $\beta$-glucan containing siRNA nanoparticles were shown to reduce gene expression of migration inhibitory factor (MIF), a cytokine and chemokine known to be upregulated in the TME in primary macrophages [170]. Upon IV injection, these nanoparticles were able to mediate a sustained reduction of MIF in tumor associated macrophages (TAMs) in a mouse model of $4 \mathrm{~T} 1$ mammary cancer $[143,171]$. Together these studies indicate that this newly characterized trafficking mechanism of $\beta$-glucan could be exploited to deliver therapeutics directly to the PDAC TME, and future studies are underway to investigate this. Ultimately, these data highlight a previously uncharacterized, dectin-1 dependent tropism of $\beta$-glucan to the pancreas that could be utilized to deliver therapeutics directly to the pancreas in the setting of cancer. 


\section{CHAPTER II}

\section{$\beta$-GLUCAN TRAFFICKING TO THE PANCREAS INCITES AN INFLUX OF \\ INNATE IMMUNE CELLS THAT SHOW A PHENOTYPE OF TRAINED}

IMMUNITY

\section{CHAPTER II INTRODUCTION}

Now that we had identified that WGP traffics to the pancreas following IP administration, we next turned our attention to investigating whether immune changes in the pancreas occurred as a result of the trafficking. It had previously been shown that IP administration of WGP causes an expansion of MPPs and LSKs in the bone marrow. We repeated this analysis to confirm that our specific form of particulate yeast-derived $\beta$ glucan had the same capability to incite central trained immunity. Unsurprisingly, WGP trained mice showed an expansion of $\mathrm{Lin}^{-} \mathrm{Sca}-1^{+} \mathrm{c}-\mathrm{Kit}^{+}(\mathrm{LSKs})$ and $\mathrm{CD} 48^{+} \mathrm{CD} 150^{-}$(MPPs) 7 days after injection in our model of trained immunity indicating the successful induction of central trained immunity (Fig 2.1A+B). Additionally, DTAF-WGP was found to traffic in small proportions to the bone marrow (Fig 2.1C). These results confirmed that the WGP used in our experiments stimulates a typical program of trained immunity. While this expansion has been noted before, the presence of trained cells within the pancreas has not been described before. Thus, the trafficking of WGP to the pancreas also caused us to question not only how the immune cell populations change, but also whether cells in the pancreas may also display features of trained immunity. 


\section{CHAPTER II RESULTS}

\section{Trafficking of immune cells into the pancreas}

After discovering that $\beta$-glucan displays tropism toward the pancreas, it was also observed that the immune landscape of the pancreas was significantly altered following WGP treatment. Arrival of WGP to the pancreas was accompanied by a distinct influx of $\mathrm{CD}_{11} \mathrm{~b}^{+}$myeloid cells to the pancreas by day 3 , some of which had phagocytosed WGP

(Fig 2.2A). This finding led us to examine how overall immune populations of the pancreas are impacted following IP WGP treatment. As it has been previously observed that the immune changes associated with trained immunity in the BM are most pronounced one week following exposure to $\beta$-glucan [131], the immune profile of the pancreas was examined in mice treated with a $3 \mu \mathrm{m}$ polystyrene microparticle or WGP 7 days following IP injection. First, it was observed that there was about a 10 -fold increase in the overall percent of $\mathrm{CD} 45^{+}$immune cells in the pancreas after WGP treatment (Fig 2.2B). There was an observed increase in the absolute number of $\mathrm{CD}^{4} 5^{+}$immune cells (Fig 2.2C). A 3 $\mu \mathrm{m}$ polystyrene microparticle control did not have such effects (Fig 2.2D), indicating that this immune cell influx is WGP specific. To further classify which cell types were responsible for this expansion of the $\mathrm{CD} 45^{+}$immune population, the relative percent (Fig 2.2B) and absolute number (Fig 2.2C) of myeloid cells $\left(\mathrm{CD} 11 \mathrm{~b}^{+}\right)$, T-cells $\left(\mathrm{CD}^{+}\right)$, B-cells $\left(\mathrm{CD} 19^{+}\right)$, and NK cells $\left(\mathrm{NK} 1.1^{+}\right)$were evaluated. The cumulative changes in the pancreas are represented by pie charts which demonstrate that the expansion of the myeloid compartment is responsible for the relative decrease in the percentage of other cell populations and the overall increase in the $\mathrm{CD} 45^{+}$population following WGP treatment (Fig 2.2E). 
We next investigated whether this influx of CD11 $\mathrm{b}^{+}$immune cells was transient, so the percent of overall $\mathrm{CD} 45^{+}$cells and $\mathrm{CD} 11 \mathrm{~b}^{+}$cells in the pancreas following IP injection was measured at 7,10, 18 and 30 days. While the percent of $C D 11 b^{+}$cells peaked at day 7, the percent of $\mathrm{CD} 45^{+}$cells appeared to peak at day 10 which may indicate that following influx of $\mathrm{CD}_{11} \mathrm{~b}^{+}$cells to the pancreas, an influx of other immune cell types may follow, though it was not investigated what type of cells these may be. The percent of immune cells in the pancreas decreased to basal levels by day 30, demonstrating that this influx is transient (Fig 2.3A). Within the myeloid compartment, we observed that the percent (Fig 2.3B) and absolute numbers (Fig 2.3C) of macrophages $\left(\mathrm{F} 4 / 80^{+}\right)$, monocytes $\left(\mathrm{Ly}_{6 \mathrm{C}}{ }^{+}\right.$), and neutrophils $\left(\mathrm{Ly}_{6 \mathrm{G}}{ }^{+}\right)$were all increased. The influx of immune cells into the pancreas was shown to be dependent on the dose of WGP injected, where higher doses of WGP were associated with increased influx of overall $\mathrm{CD}_{4} 5^{+} \mathrm{CD} 11 \mathrm{~b}^{+}$myeloid cells and $\mathrm{CD} 45^{+} \mathrm{CD} 11 \mathrm{~b}^{+} \mathrm{F} 4 / 80^{+}$macrophages (Fig 2.4). We also wanted to ensure that this observed phenomenon was not specific to C57BL/6 mice, and so investigated whether other commonly used mouse strains, such as BALB/c mice, also showed an influx of immune cells into the pancreas following WGP injection. BALb/c mice also showed a 15 fold increase in CD45+ cells in the pancreas after WGP injection (Figure 2.5).

As pancreatitis and the destruction of pancreatic islets are associated with immune cell infiltration of the pancreas, $\mathrm{H}+\mathrm{E}$ of the pancreas was performed to assess the integrity of pancreatic islets 7 days after WGP administration (Fig 2.6A). Serum amylase, a diagnostic marker of pancreatitis, was also measured in WGP vs PBS-treated mice 7 days following injection (Fig 2.6B). Neither the islets nor the serum amylase were adversely impacted by WGP treatment and the observed immune influx, indicating that the immune cell influx in 
this mechanism does not cause pancreatic destruction. Further, following WGP treatment mice were monitored for up to 3 months, and no morbidities or mortalities were associated with WGP treatment.

As the islets were not adversely impacted by the influx of immune cells, we wondered where the immune cells were located. H+E imaging of PBS and WGP treated mice showed that the cellular infiltrates appeared to be located in a layer around the pancreas that was rich in adipose tissue, and were starkly increased in WGP treated mice (Fig 2.7A+B). This layer was connected to the pancreas and was identified as being a part of the serosa capsule that surrounds the pancreas.

\section{Trained Immunity in the Pancreas}

To investigate whether the cells found to infiltrate the mouse pancreas following WGP treatment displayed a phenotype of trained immunity, we used a standard training protocol in which mice were treated with WGP or PBS and 7 days later the pancreas were harvested, processed into a single cell suspension, and then restimulated with LPS. TNF- $\alpha$ production has been used as a surrogate marker to evaluate the trained response. Overall CD11 $\mathrm{b}^{+}$ myeloid cells, $\mathrm{CD} 11 \mathrm{~b}^{+} \mathrm{F} 4 / 80^{+}$macrophages and $\mathrm{CD} 11 \mathrm{~b}^{+} \mathrm{Ly} 6 \mathrm{C}^{+}$monocytes were all shown to produce more TNF- $\alpha$ due to prior exposure to WGP, as assessed by the percent of TNF$\alpha^{+}$cells and the MFI (Fig 2.8). To further assess these findings, CD11 $b^{+}$cells were sorted from the pancreas of these mice and restimulated ex-vivo with LPS, and the TNF- $\alpha$ and IL-

6 levels in the supernatants were measured by ELISA. As compared to CD11 $b^{+}$cells from PBS mice, cells from WGP-trained mice that were restimulated with LPS produced significantly more TNF- $\alpha$ and IL-6 (Fig 2.9A), signifying that WGP treated pancreatic 


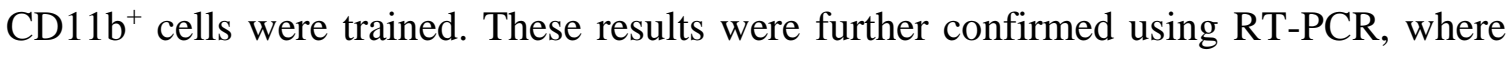
pancreatic $\mathrm{CD} 11 \mathrm{~b}^{+}$cells sorted from PBS or WGP-trained mice were shown to produce more TNF- $\alpha$, IL-6, iNOS and less IL-10 due to WGP treatment (Fig 2.9B). Together these findings indicate that not only does WGP incite a trained phenotype in the cells that traffic into the pancreas, but that they also have an overall pro-inflammatory phenotype.

Some reports have indicated that the microbiota of mice can play a role in the induction of trained immunity $[172,173]$. The microbiome of mice can be drastically different between facilities and breeding colonies, and so we wanted to confirm that the induction of trained immunity in the pancreas was not dependent on factors related to the microbiome of the mice in our facility [174-176]. To investigate this, germ-free mice housed in the germ-free facility at the University of Louisville were injected IP with WGP and the induction of trained immunity in pancreatic myeloid cells was assessed (Fig 2.10). Myeloid cells from WGP-treated germ-free mice did display increased production of $\mathrm{TNF} \alpha$ as compared to PBS-treated mice, indicating that while the microbiota may play a role in trained immunity, it was not a critical factor in this model.

\section{RNA-Seq and CyTOF Analysis of WGP-induced influx of myeloid cells}

RNA sequencing (RNA-Seq) was then performed on FACS sorted CD45 ${ }^{+} \mathrm{CD} 11 \mathrm{~b}^{+}$cells 7 days following PBS or WGP training to obtain an unbiased and comprehensive characterization of myeloid populations in the pancreas. A total of 1459 Differentially Expressed Genes (DEGs) were discovered, with 661 upregulated and 798 genes downregulated in the WGP-trained setting (Fig 2.11A). In accordance with the observed influx of immune cells, the DEGs related to leukocyte chemotaxis, leukocyte migration 
monocyte chemotaxis, macrophage migration, mononuclear cell migration, and myeloid leukocyte migration (Fig 2.11B) were all significantly enriched. Gene set enrichment analyses (GSEA) further confirmed previously investigated upregulations in pathways related to TNF- $\alpha$ cytokine production, IL-6 production, and nitric oxide production (Fig 2.11C). Additionally, Reactome pathways CLEC7A dectin-1 signaling and C-type lectin receptor pathways were significantly enriched, further corroborating the Dectin-1 involvement in this process (Fig 2.11D).

Considering that the myeloid population was observed to be responsible for the expansion of the $\mathrm{CD} 45^{+}$population and that myeloid cells are a diverse population, $\mathrm{CyTOF}$ analysis was performed on pancreata from mice treated with PBS or WGP 7 days prior. Cell populations within the pancreas were identified and compared between treatment groups; t-SNE plots indicate that following WGP treatment, numerous new populations of myeloid cells are present in the pancreas due to WGP treatment (populations 11, 2, 6, 8, 9, $12,13,14,15$, and 17) which though heterogenous were characteristically defined by the surface expression of Ly6C, F4/80, MHCII, CCR2, CD80, CD86 and PD-L1, indicative of activated inflammatory macrophages and monocytes. These populations are highlighted

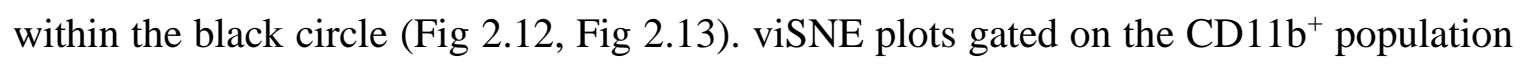
showed a distinct expansion of monocytes and macrophages, with additional increases of the Dendritic Cell (DCs), neutrophil, and NK populations after WGP treatment (Fig 2.14A). Additionally, these analyses highlight that following WGP training and LPS restimulation, TNF $\alpha$ was primarily produced by macrophages and monocytes, IL-6 and iNOS were primarily produced by macrophages, Granzyme-B was produced by NK-cells and macrophages, and IFN $\gamma$ was principally produced by neutrophils (Fig 2.14B). This 
reveals that while many cell populations are changed due to WGP treatment, the primary cells trained by WGP, as assessed by increased TNF $\alpha$ expression, are macrophages and monocytes and that there is not a singular cell type responsible for the phenotype of the trained immunity that was observed.

\section{Myeloid cells in the pancreas are trained independently of adaptive immune}

\section{responses}

To assess whether this phenomenon of myeloid cell influx and activation was dependent upon other immune populations or driven entirely by the myeloid cells themselves, we depleted specific immune populations and observed the trained phenotype. Anti-CD4 and anti-CD8 mAbs were used alone and in combination to deplete T-cells, the efficiency of depletion was assessed (Fig 2.15A), and the trained phenotype of myeloid cells was observed (Fig 2.15B+C). NK cells were depleted using mAb PK136, the depletion efficiency was evaluated (Fig 2.16A) and the trained phenotype was observed (Fig 2.16B). In these depletion conditions, myeloid cells were still observed to be trained by WGP. The trained phenotype was also assessed in NSG mice which lack B-cells, Tcells, and NK cells (Fig 2.17). These mice still showed a clear phenotype of trained immunity. We also depleted neutrophils using Ly6G mAb. Despite depletion of granulocytes, we still observed WGP-induced training in the $\mathrm{CD}_{11 \mathrm{~b}^{+}}$myeloid compartment (Fig 2.18). Taken together, our data support that the infiltrating myeloid cells in the pancreas are trained by $\beta$-glucan without the assistance or influence of adaptive immune populations, NK cells, or granulocytes. 


\section{A single-cell RNA sequencing reveals specific populations of pro-inflammatory macrophage/monocytes that traffic to the pancreas upon $\beta$-glucan treatment}

To gain a more in-depth understanding of the cell populations present in the pancreas after WGP treatment, a single-cell RNA sequencing (scRNA-Seq) was performed on sorted $\mathrm{CD}^{+} 5^{+}$cells from PBS-treated mice and mice treated with WGP three (3-day WGP) and seven days prior (7-day WGP). Two dimensional UMAP representation of 11,132 cells aggregated from three samples (PBS=4213, 3-day WGP $=3757$, 7-day WGP=3244) with clusters resulting from k-nearest neighbors and Louvain algorithms partitioned into 19 distinct clusters (Fig 2.19A+B). The relative frequency of each cluster within each experimental group was assessed. Here, significant increases in the frequency of clusters 3,4 , and 10 were observed by day 7 after WGP treatment, along with a near disappearance of cluster 5 (Fig 2.19C). The relative frequencies of several other populations were also shown to decrease over time. However, this apparent trend is likely due to the relative increase in frequency of other populations. Overall, clusters 3,4,10 and 5 appear most significantly altered due to WGP treatment (2.19C)

The populations noted previously to be most significantly altered over time due to WGP treatment were part of the myeloid compartment, as identified by CSF1R expression (Fig 2.20A+B). As these data along with previous findings suggests that myeloid populations drive the immune changes associated with WGP treatment, the dynamic $C S F 1 R^{+}$clusters $3,4,10$ and 5 were investigated in more detail as shown by UMAP and violin plots (Fig 2.21A+B) and dot plots representing the top 12 marker genes by average $\mathrm{Z}$-score for each cluster (2.22A). Cluster 5, which was present in PBS mice but virtually disappeared after WGP treatment was ITGAM ${ }^{\mathrm{Int}} A D G R E 1^{\mathrm{Hi}} \mathrm{Lyz} 2^{\mathrm{Hi}} H 2-A b 1^{\mathrm{Hi}} L y 6 C 2^{-}$and did not express 
TNFAIP2, indicating that these cells are resident macrophages $[177,178]$. This cluster also notably expressed $M A F$ and $A P O$ which are both related to M2 macrophage polarization [179-181]. Cluster 19 was largely unchanged over time so was not described in more detail, but exhibited an expression profile similar to cluster 5, and thus also represents a subset of tissue resident macrophages. Cluster 10 was characterized as ITGAM ${ }^{\mathrm{Hi}} A D G R E 1^{H i} L y z 2^{\mathrm{Hi}}$ $H 2-A b 1^{I n t} L y 6 C 2^{-}$with notable expression signatures of $A R G 1$ and $F A B P$. Clusters 3 and 4 share general phenotypic characterization as ITGAM ${ }^{\mathrm{Int}} A D G R E 1^{\text {Int }} L y z 2^{\mathrm{Hi}} H 2-A b 1^{\text {Int }}$ $L y 6 C 2^{\mathrm{Hi}} C C R 2^{\mathrm{Hi}}$, which suggests that both are subsets of infiltrating monocytes/macrophages. Cluster 4 had notable expression of Chil3 and Plac8, which together have been identified by another group to identify Ly6C $\mathrm{C}^{\text {hi }}$ infiltrating macrophages in the kidney [182]. Finally, cluster 3, which was absent in naïve mice and showed the greatest increase in relative frequency among all clusters following WGP treatment expressed substantial signatures of TNFAIP2, ILIB, SOD2, and PRDX5, indicating a strongly pro-inflammatory phenotype. Cluster 3 was thus identified as inflammatory infiltrating monocytes/macrophages. Interestingly, within the myeloid subset, the populations shown to increase due to WGP treatment are the only populations to express TNFAIP2, indicating that our previous use of $\mathrm{TNF} \alpha$ as a surrogate marker of trained immunity was accurate.

To further characterize the activation status of each of the four dynamic myeloid clusters, dot plots were constructed to show the relative expression of genes associated with a pro-inflammatory status and an anti-inflammatory status (2.22B). Clusters 3 and 4 , the infiltrating monocytes/macrophages, were shown to be pro-inflammatory, the resident macrophages were anti-inflammatory, and the macrophages in cluster 10 showed an 
intermediate phenotype. In agreement with previous CyTOF and flow cytometry data, this scRNA-Seq data further characterize the newly identified pro-inflammatory monocytes and macrophages in the pancreas due to WGP training. 


\section{CHAPTER II FIGURES}
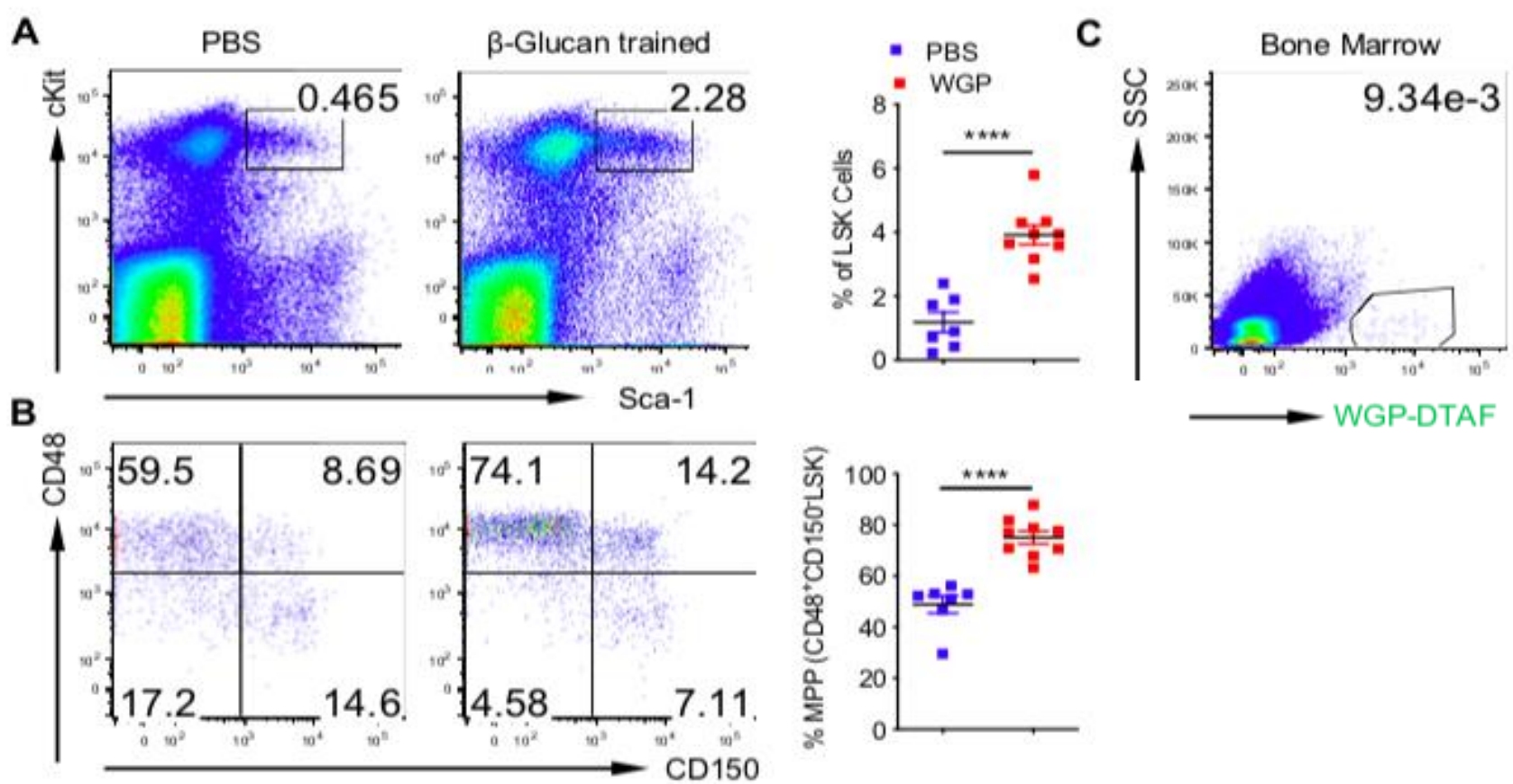

Figure 2.1: IP WGP traffics to the bone marrow and causes an expansion of myeloid progenitor cells

The bone marrow of mice injected with IP WGP or PBS was analyzed for (A) $\mathrm{Lin}^{-} \mathrm{Sca}-1^{+} \mathrm{c}-$ $\mathrm{Kit}^{+}$(LSKs) and (B) CD48 CD150- MPPs 7 days after injection. (C) DTAF WGP was injected IP and 3 days later the bone marrow was assessed for the presence of WGP. Data are represented as mean \pm SEM. $* * * * p<.0001$ 

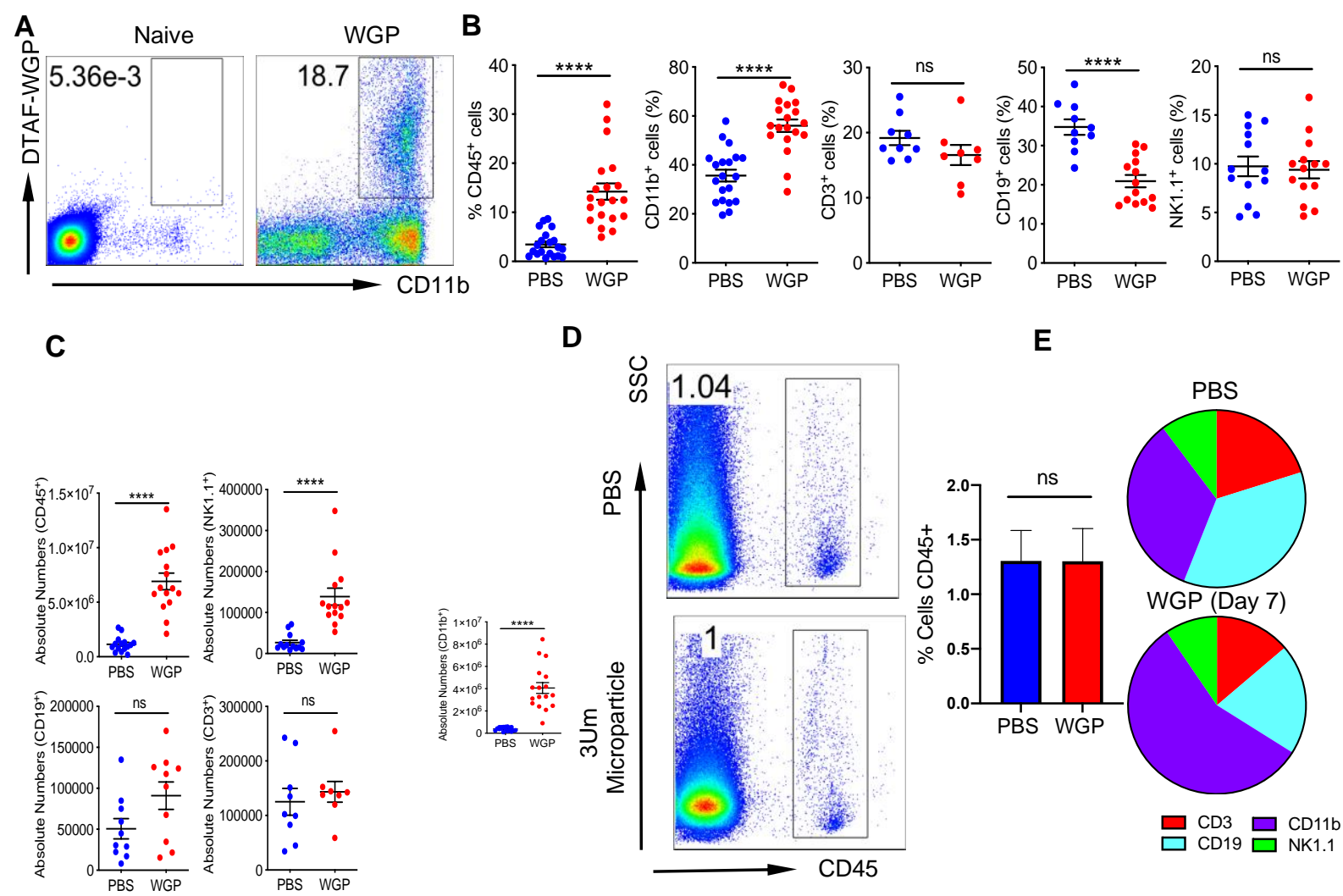

D

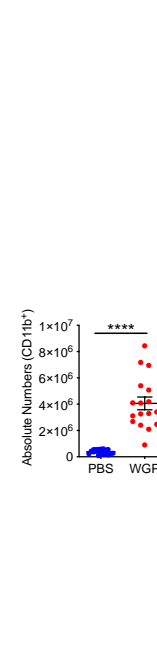

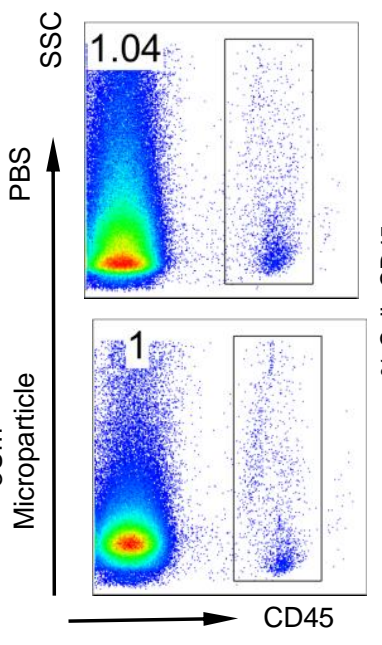

E

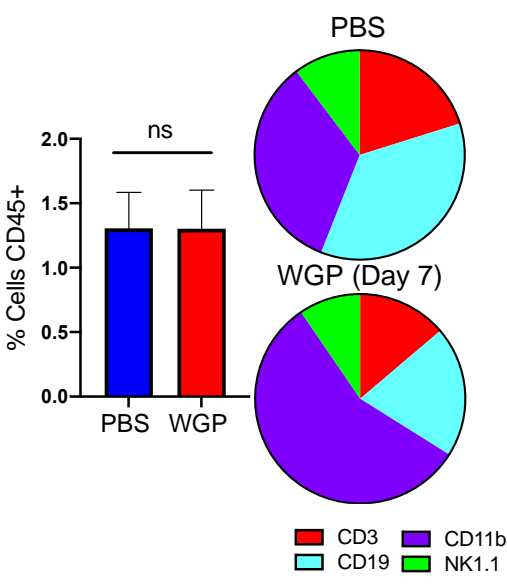

Figure 2.2: IP WGP causes an influx of CD11b+ myeloid cells into the pancreas

(A) WT mice were treated with I.P. DTAF-WGP and 3 days later the percent of

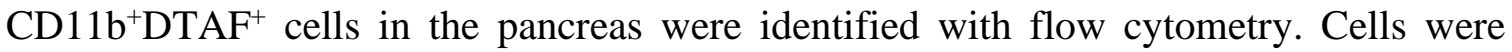
previously gated on $\mathrm{CD}^{4} 5^{+}$. (B) 7 days after I.P. PBS or WGP injection (7-day WGP), the percent of $\mathrm{CD}_{4} 5^{+}$cells in the pancreas were identified. The percent of $\mathrm{CD} 11 \mathrm{~b}^{+}, \mathrm{CD}^{+}$, $\mathrm{CD} 19^{+}$and $\mathrm{NK} 1.1^{+}$cells within the $\mathrm{CD} 45^{+}$population were also measured. (C) The absolute number of $\mathrm{CD}_{4} 5^{+}$immune cells, NK1.1 ${ }^{+} \mathrm{NK}$ cells, $\mathrm{CD} 19^{+} \mathrm{B}$-cells, $\mathrm{CD} 3^{+} \mathrm{T}$-cells, CD11 ${ }^{+}$myeloid cells in PBS and 7-day WGP trained mice. (D) $3 \mu \mathrm{m}$ polystyrene beads or PBS were injected IP and 7 days later the percent of CD45+ cells in the pancreas were assessed. (E) Pie charts representing the relative change in frequency of each major 
immune cell population between the PBS vs 7-day WGP treatment setting. Data are represented as mean \pm SEM. ns $=$ not-significant; $* * * * p<.0001$ 
A

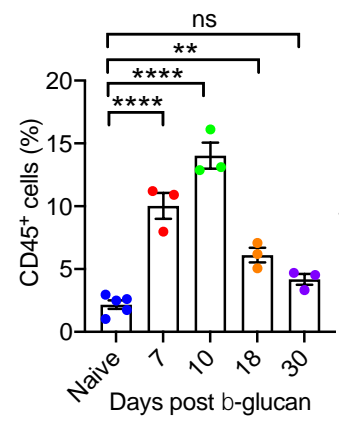

B

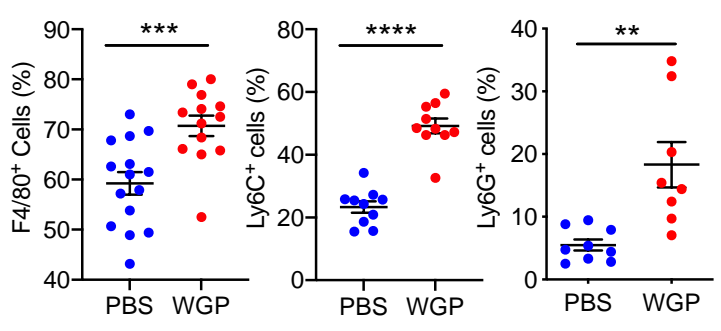

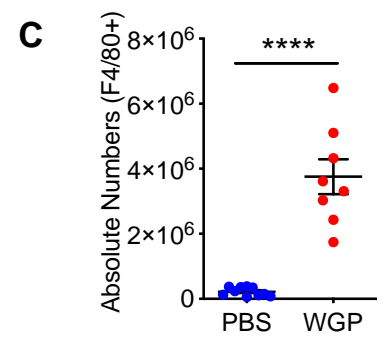
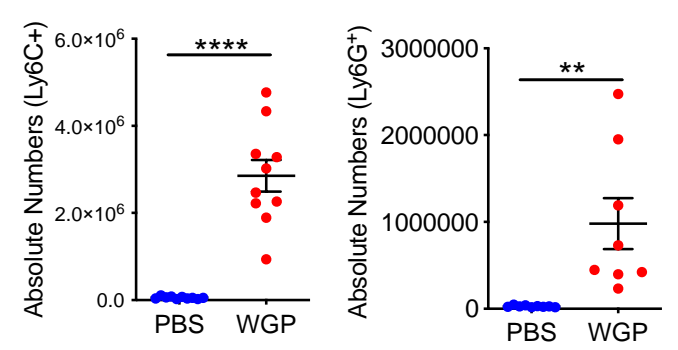

Figure 2.3: A transient influx of diverse myeloid cell subtypes occurs after WGP treatment

(A) WT mice were injected with WGP and the percent of $\mathrm{CD}_{4} 5^{+}$and $\mathrm{CD} 45^{+} \mathrm{CD} 11 \mathrm{~b}^{+}$cells were measured 7, 10, 18, and 30 days later. These were compared to PBS treated mice. (B) Gated on the pancreatic $\mathrm{CD} 45^{+} \mathrm{CD} 11 \mathrm{~b}^{+}$population, the percent of $\mathrm{F} 4 / 80^{+}$(macrophages), Ly6C $^{+}$(monocytes) and Ly6G ${ }^{+}$(neutrophils) were measured using flow cytometry. (C) The absolute number of $\mathrm{CD} 11 \mathrm{~b}^{+} \mathrm{F} 4 / 80^{+}$macrophages, $\mathrm{CD} 11 \mathrm{~b}^{+} \mathrm{Ly} 6 \mathrm{C}^{+}$monocytes, and

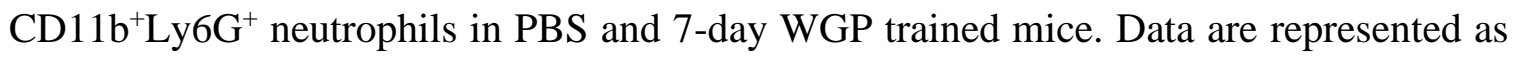
mean \pm SEM. ns= not-significant ${ }^{*} p<.05, * * p<.01, * * * p<.001, * * * * p<.0001$ 

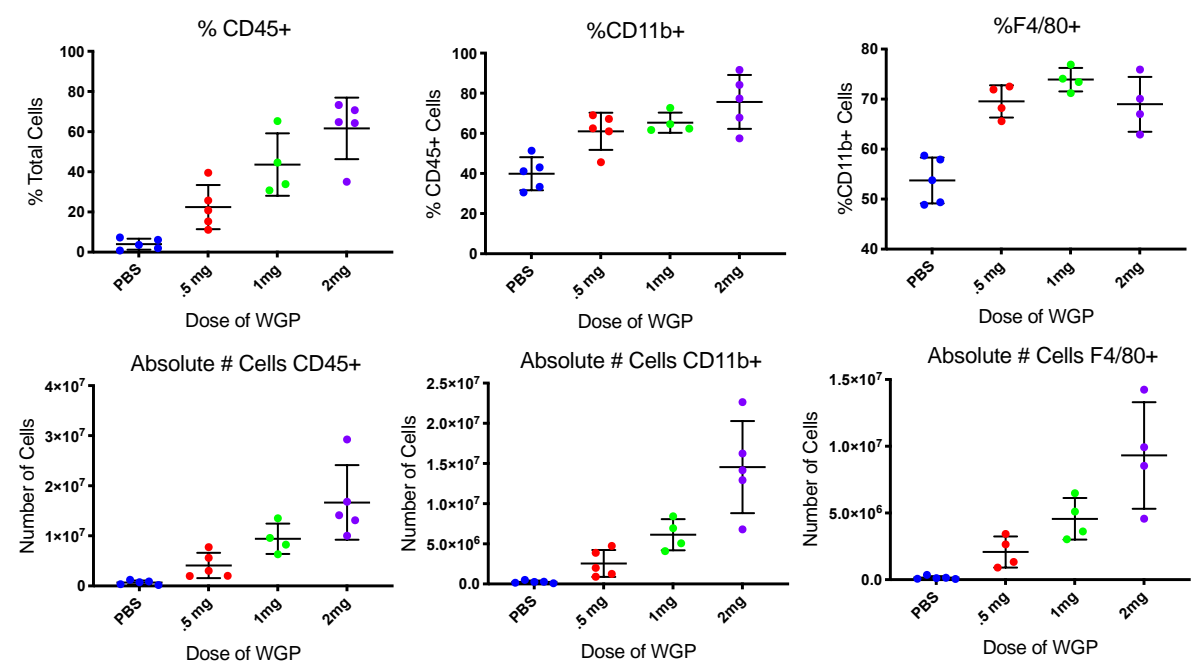

Figure 2.4: The trafficking of immune cells into the pancreas is dose dependent

The percent and absolute number of $\mathrm{CD} 45^{+}, \mathrm{CD} 45^{+} \mathrm{CD} 11 \mathrm{~b}^{+}$and $\mathrm{CD} 45^{+} \mathrm{F} 4 / 80^{+}$cells in PBS mice and mice treated with $.5,1$ and $2 \mathrm{mg}$ of WGP.
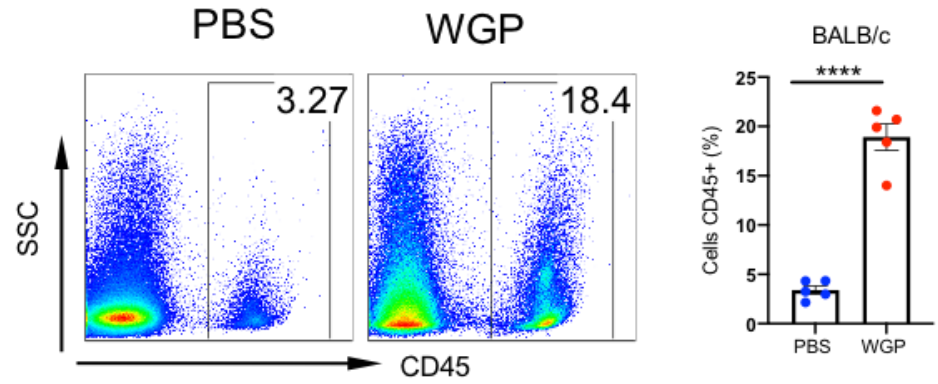

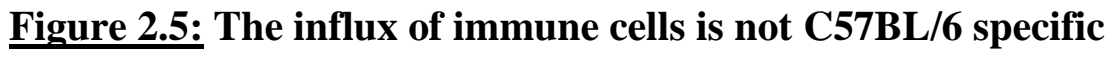

Balb/c mice were given IP WGP and 7 days later the pancreata was harvested and the percent of CD45+ cells were investigated. Data are represented as mean \pm SEM., $* * * * p$ $<.0001$ 
A

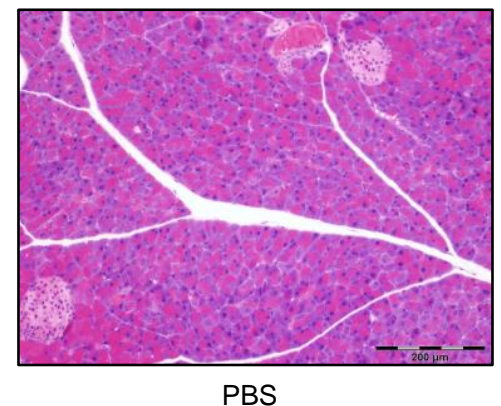

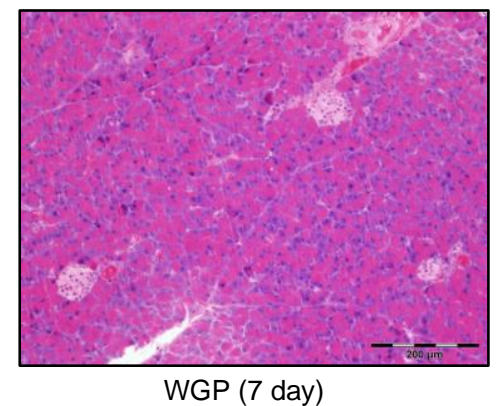

B

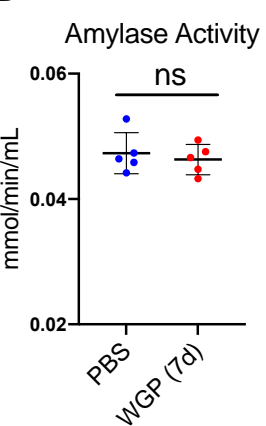

Figure 2.6: The WGP-driven influx of immune cells does not harm the pancreas

(A) A representative section from $\mathrm{H}+\mathrm{E}$ imaging of the pancreas from PBS and 7-day WGP trained mice. (B) The levels of amylase in the serum of PBS and 7-day WGP trained mice. Data are represented as mean \pm SEM. ns= not-significant; 
A
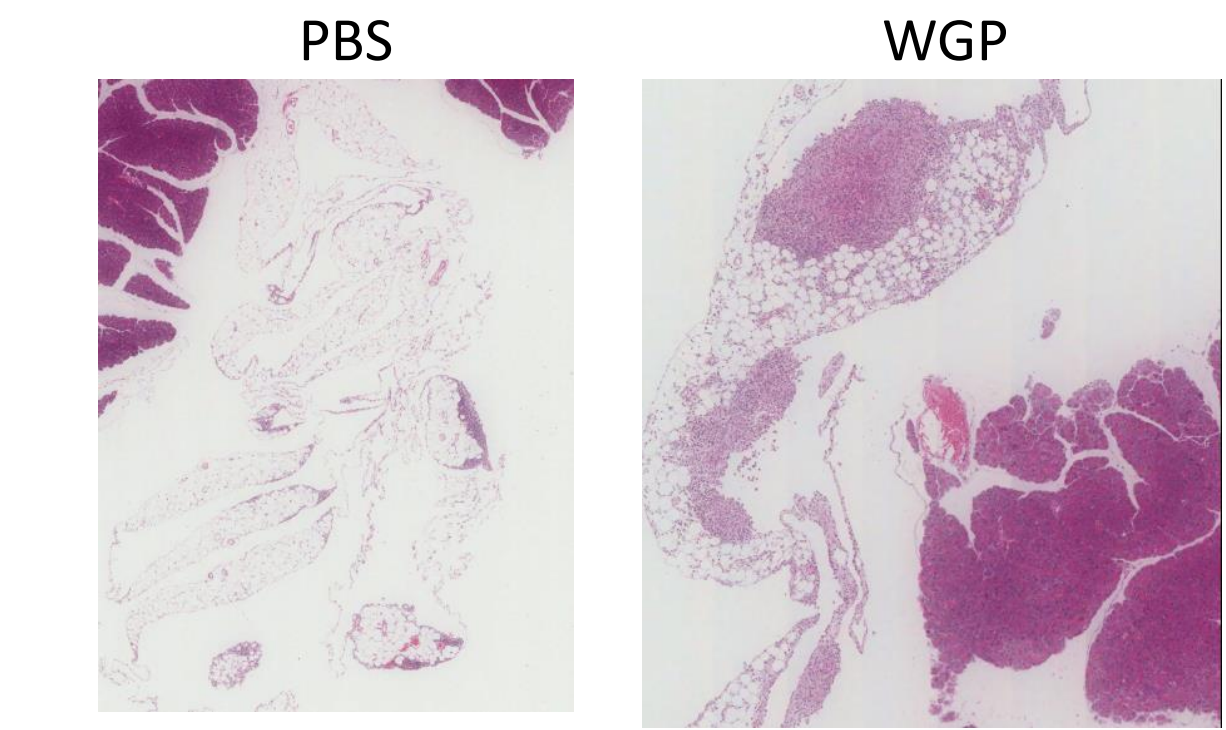

B
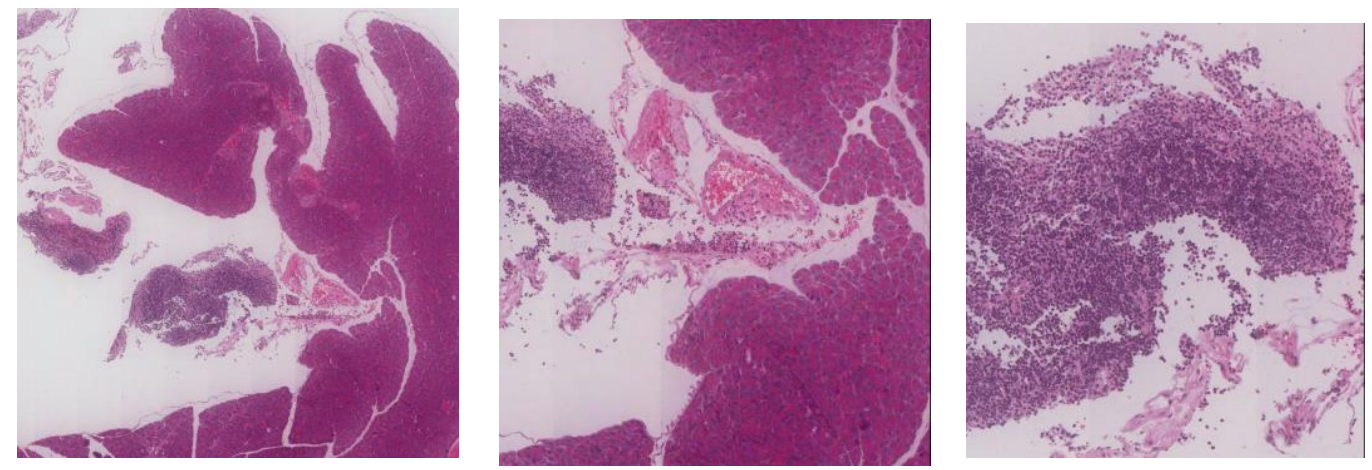

Figure 2.7: H+E imaging of PBS and WGP treated pancreases

(A) Mice were treated with PBS or WGP and 7 days later the pancreases were harvested and analyzed to determine the location of the immuneological influx. (A) PBS v WGP treated pancreases highlighting the fatty viscera that appears to be the target of immune infiltration (B) Magnified imaging of immune infiltrates in WGP treated mice. 


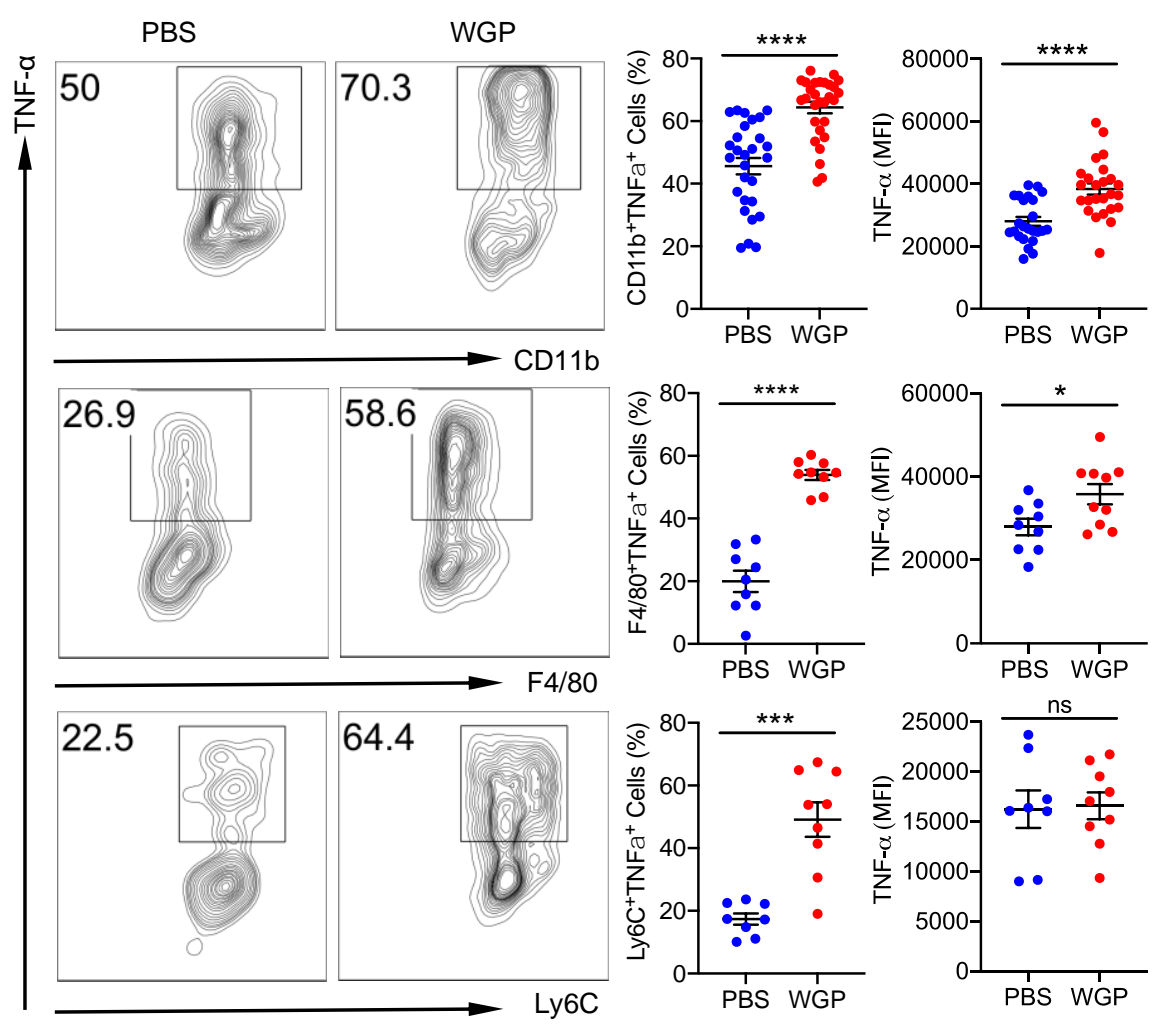

Figure 2.8: Myeloid cells in the pancreas display features of trained immunity

Mice were trained with PBS or WGP. 7 days later mouse pancreas were harvested and

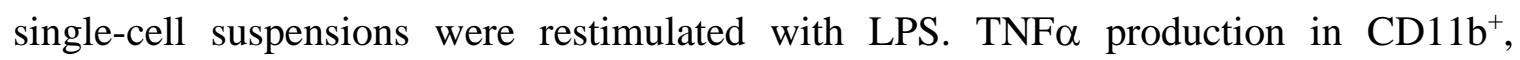
$\mathrm{CD}_{11} \mathrm{~b}^{+} \mathrm{F} 4 / 80^{+}$, and $\mathrm{CD} 11 \mathrm{~b}^{+} \mathrm{Ly} 6 \mathrm{C}^{+}$cells were measured using intracellular staining by flow cytometry. Data are represented as mean \pm SEM. ns $=$ not-significant; ${ }^{*} p<.05$, $* * p$ $<.01, * * * p<.001, * * * * p<.0001$ 
A
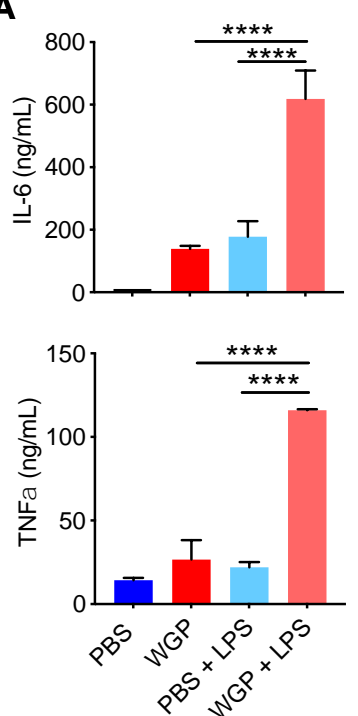
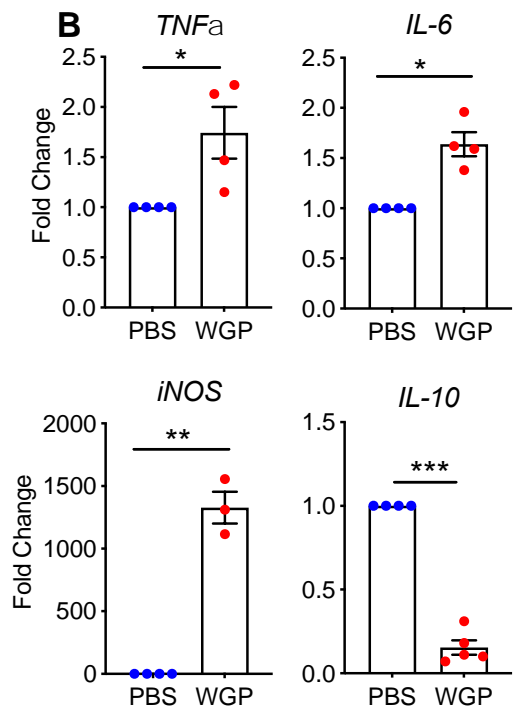

Figure 2.9: Enriched CD11b+ pancreatic cells show a phenotype of trained immunity (A) 7 days after PBS or WGP training, the $\mathrm{CD} 45^{+} \mathrm{CD} 11 \mathrm{~b}^{+}$population was enriched using FACS sorting. Cells were plated and restimulated with PBS or LPS for 24 hours and the TNF $\alpha$ and IL-6 in the supernatant were measured using an ELISA. (B) Pancreatic CD11b ${ }^{+}$ cells from PBS and WGP-trained mice were sorted using FACS and RT-qPCR was done to quantify TNF $\alpha$, IL-6, iNOS and IL-10 mRNA expression levels. Data are represented as mean \pm SEM. ns $=$ not significant ${ }^{*} p<.05, * * p<.01, * * * p<.001, * * * * p<.0001$ 

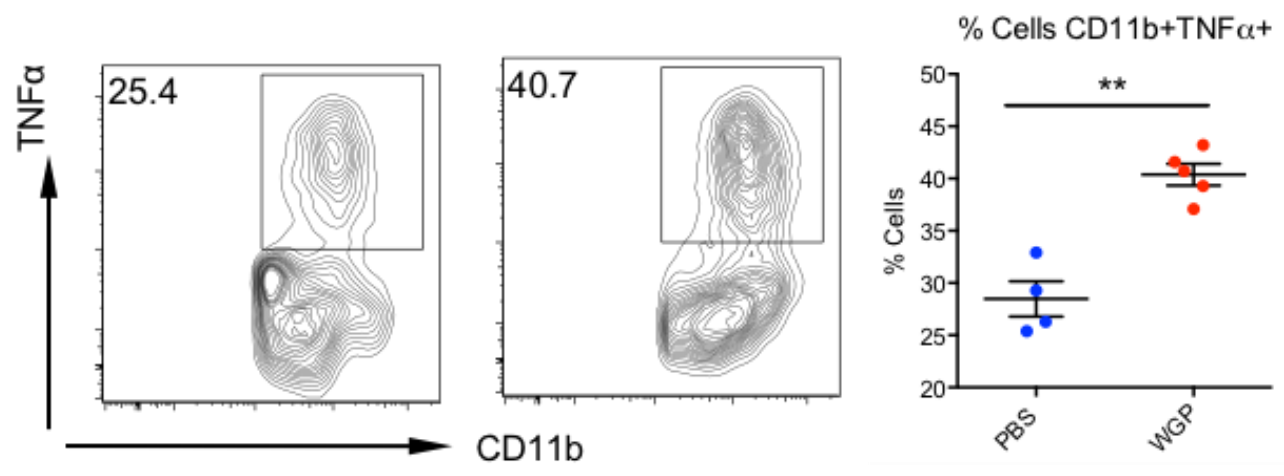

\section{Figure 2.10: the induction of trained immunity in germ-free mice}

Germ-free animals bred and maintained in the university of Louisville germ-free animal facility were injected with WGP and 7 days later the pancreatic myeloid cells were restimulated with LPS and the production of $\mathrm{TNF} \alpha$ was assessed. Data are represented as mean \pm SEM. $* * p<.01$ 
A

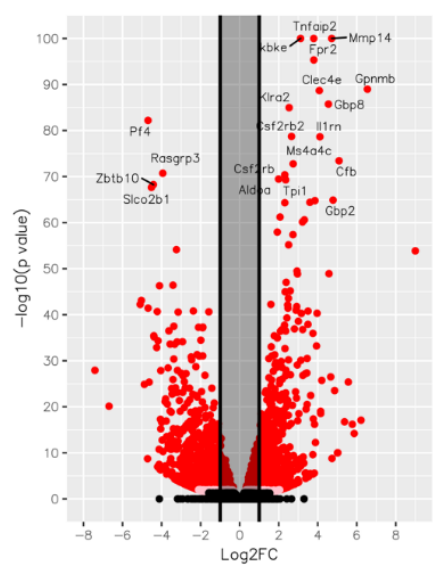

C

$\mathrm{GO}+$ regulation of TNF $\alpha$ superfamily cytokine production

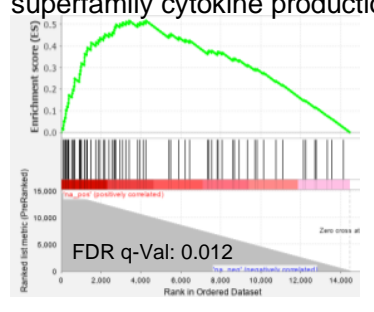

B

GO_Monocyte Chemotaxis

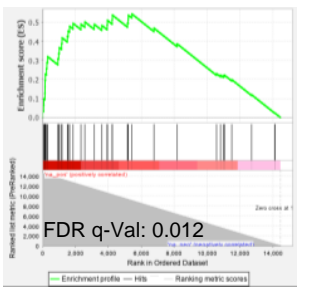

GO_Mononuclear Cell Migration

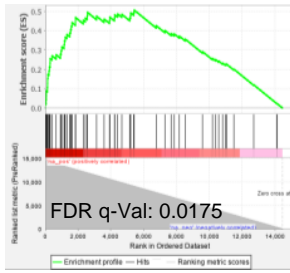

GO_IL-6 production

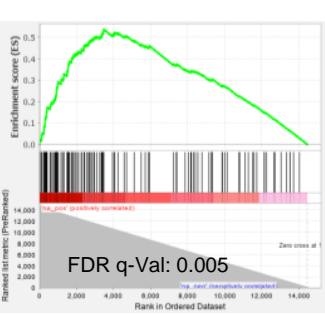

GO_Macrophage Migration

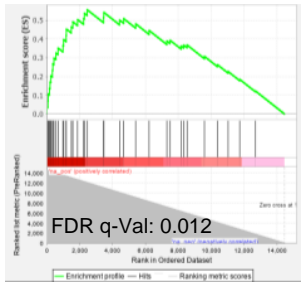

GO Myeloid Leukocyte Migration
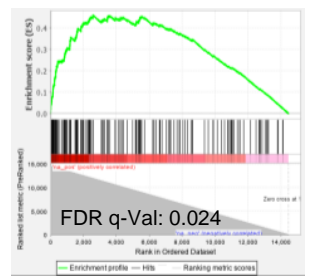

D

Reactome_CLEC7A

Dectin-1 Signaling

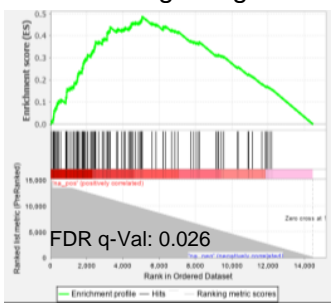

GO_Leukocyte Chemotaxis

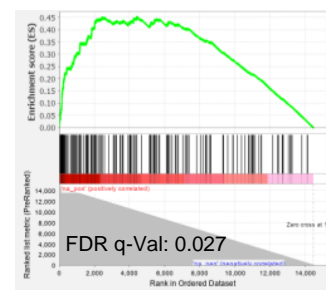

GO_Leukocyte Migration

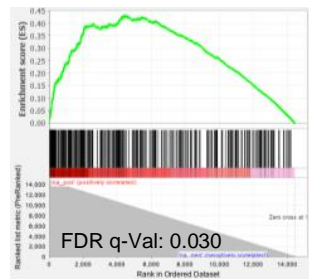

Reactome_C-Type Lectin Receptors (CLRS)

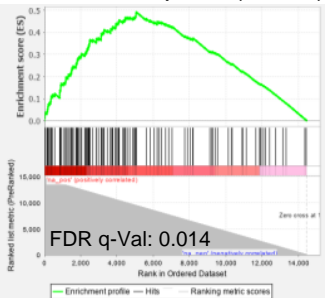

\section{Figure 2.11: RNA-Seq analysis of PBS versus WGP treated pancreatic CD11b+ cells}

Mice were trained with PBS or WGP and 7 days later the $\mathrm{CD} 45^{+} \mathrm{CD} 1 \mathrm{~b}^{+}$populations were sorted and RNA-Seq analysis was performed (PBS n=3, WGP n=3). (A) The distribution of $p$ values $\left(-\log _{10}(p\right.$ value $\left.)\right)$ and fold changes $\left(\log _{2} \mathrm{FC}\right)$ of differentially expressed genes in WGP vs PBS trained cells are shown in a volcano plot. (B-D) Enrichment plots generated by Gene Set Enrichment Analysis (GSEA) for genes related to (B) monocyte chemotaxis, mononuclear cell migration, macrophage migration, leukocyte migration, leukocyte chemotaxis, and leukocyte migration, (C) TNF $\alpha$ and IL-6 production, and (D) Dectin-1 signaling in CD11b ${ }^{+}$cells from 7-day WGP-trained as compared to PBS mice. 
PBS

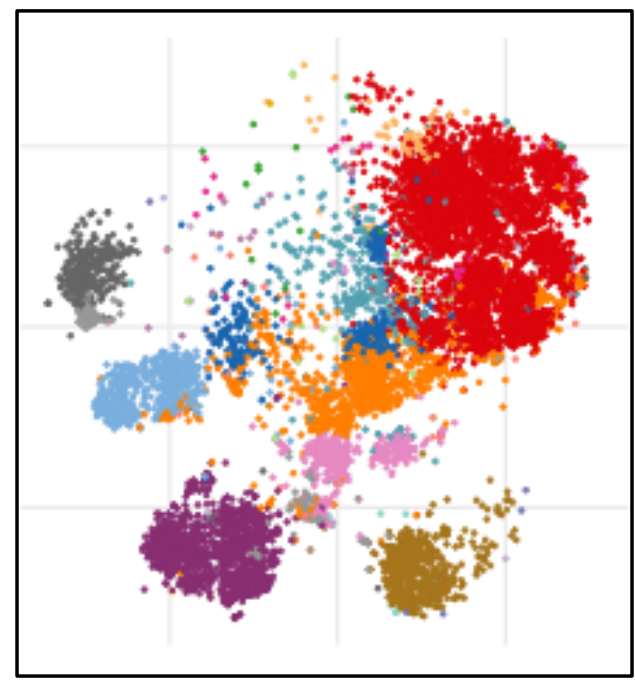

WGP (7 day)

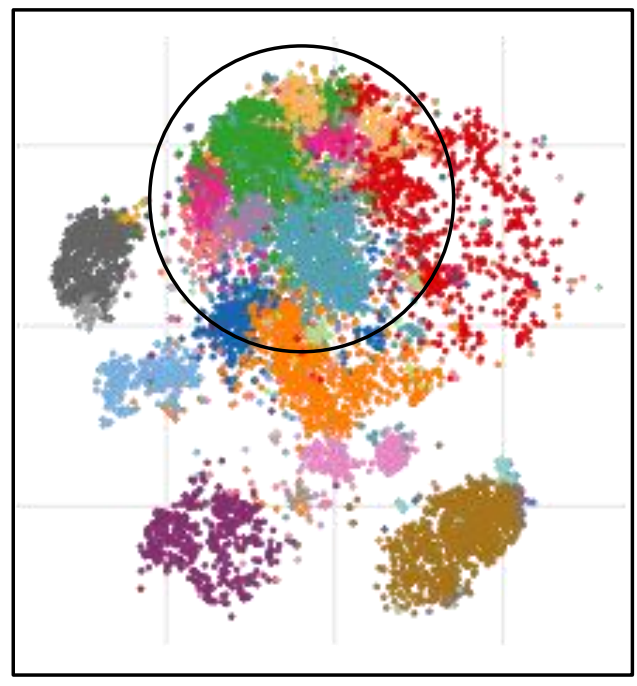

\begin{tabular}{lll}
1 & 1 \\
\hdashline & 2 & 11
\end{tabular}

0

- 4 14

- $5 \bigcirc 15$

$6 \quad 16$

$7 \bigcirc 17$

8 18

$\begin{array}{lll}9 & 9 & 19\end{array}$

\section{Figure 2.12: Characterization of the pancreatic $\mathrm{CD}_{11 b^{+}}$population using CyTOF}

t-SNE plots of the CD11 $\mathrm{b}^{+}$population in mice trained with PBS or WGP 7 days prior and analyzed with CyTOF (PBS n=3, WGP $n=3$ ). The black circle highlights new cell populations in the pancreas following treatment with WGP 


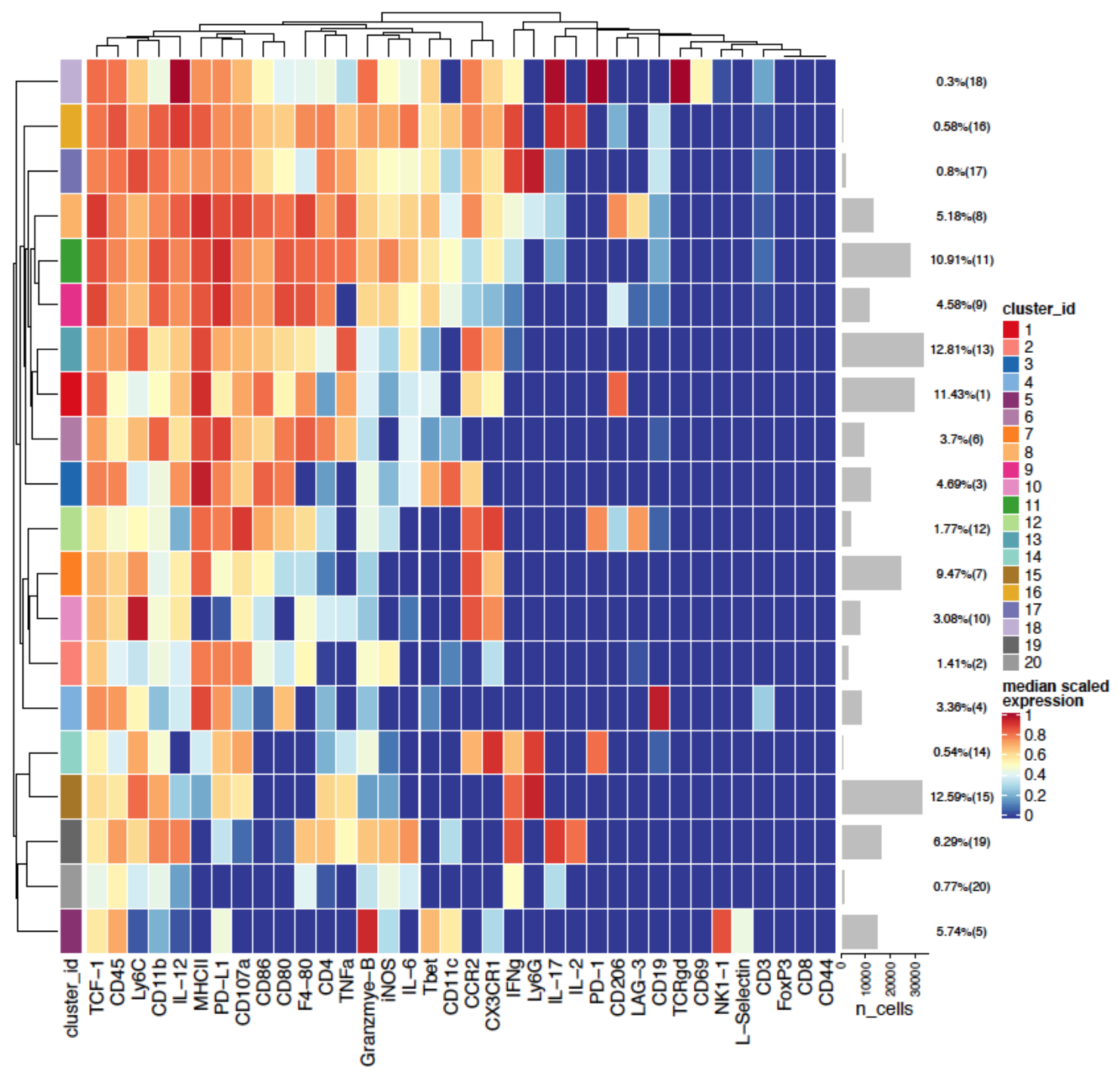

Figure 2.13: Heatmap of the median scaled expression of the CyTOF markers in each cluster determined from CyTOF workflow. 
A

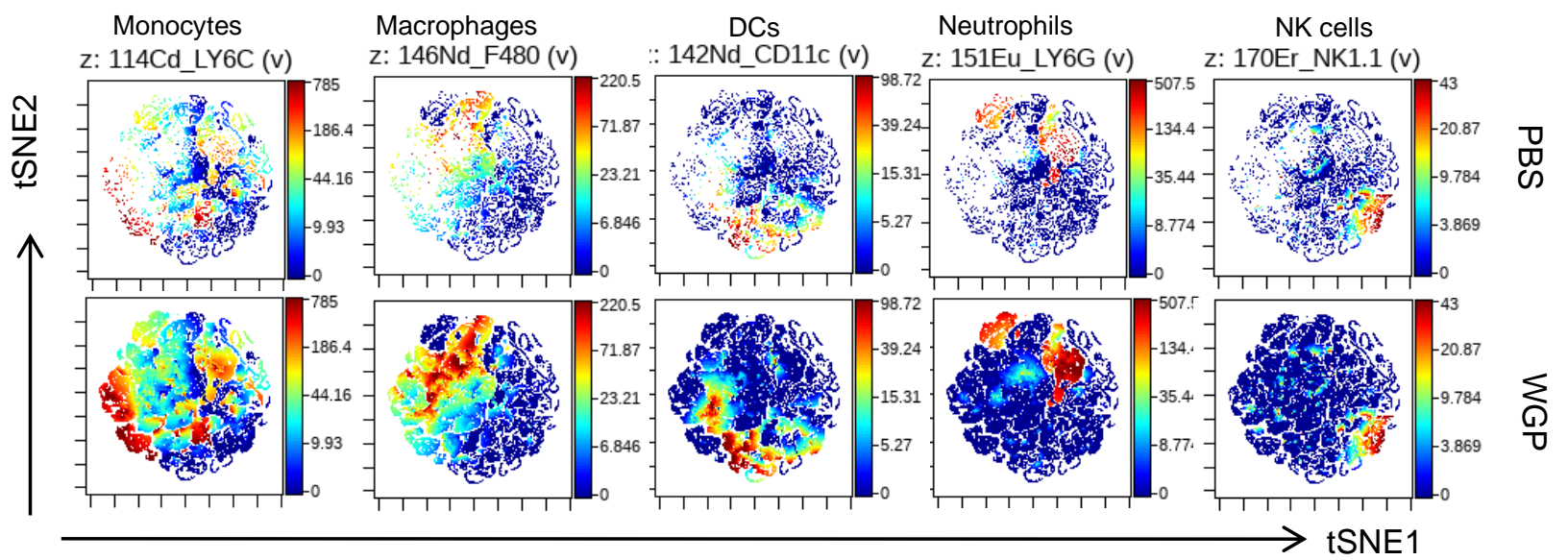

B

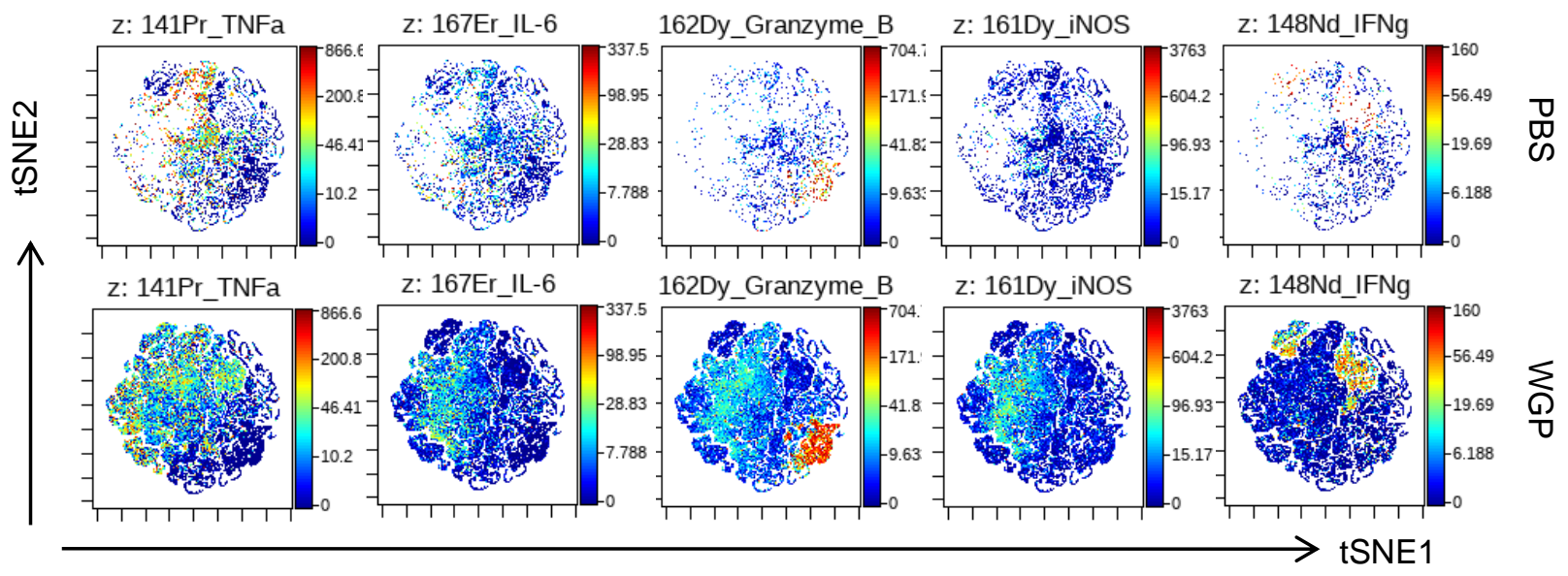

Figure 2.14: Functional characterization of pancreatic CD11b+cells sing CyTOF

(A) viSNE plots of $\mathrm{CD}_{11 b^{+}}$pancreatic cells in PBS and 7-day WGP trained mice, made from CyTOF data, highlighting the specific expression of Ly6C, F4/80, CD11c, Ly6G and NK1.1 in order to indicate the relative location of the major immune-cell subsets and changes between the PBS and WGP treatment group. (B) viSNE plots of CD11b ${ }^{+}$ pancreatic cells in PBS and 7-day WGP trained mice, made from CyTOF data, showing the expression of TNF $\alpha$, IL-6, Granzyme-B, iNOs and IFN $\gamma$. 

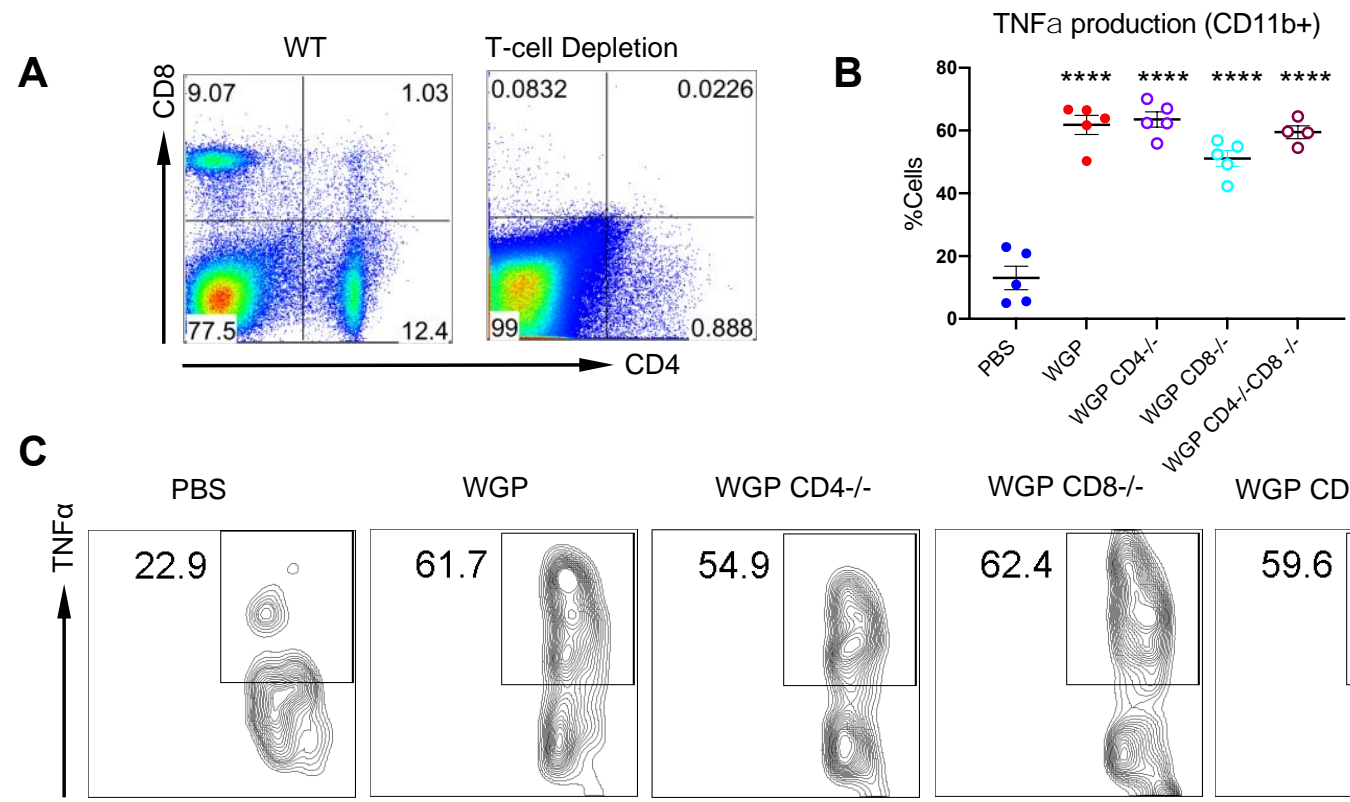

WGP

WGP CD4-/-

WGP CD8-/-

WGP CD4-/-CD8-/-
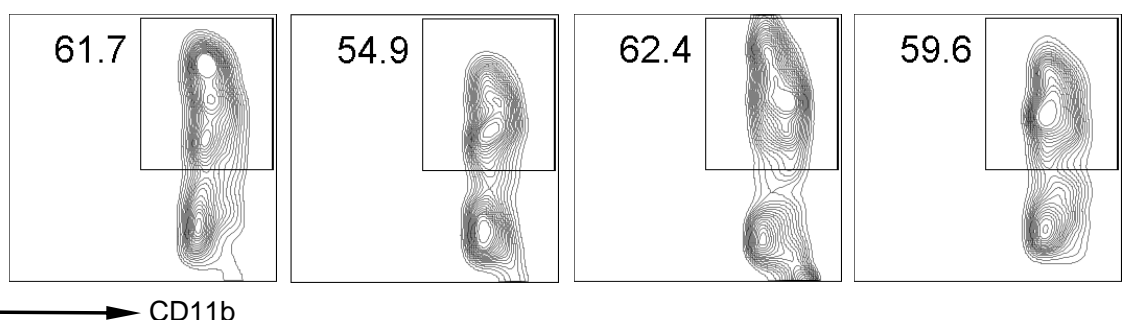

Figure 2.15: Training of pancreatic myeloid cells is not dependent on T-cells

(A) Representation of the T-cell depletion efficiency in the spleen of anti-CD-4 and cntiCD-8 mAbs used in vivo. $(\mathbf{B}+\mathbf{C})$ Mice depleted of CD4 ${ }^{+}$T-cells, CD8 ${ }^{+}$T-cells or both $\mathrm{CD}^{+}$and $\mathrm{CD} 8^{+}$T-cells and WT mice were given I.P. WGP and 7 days later the pancreatic cells from these mice and WT mice treated with PBS were restimulated with LPS. TNF $\alpha$ production in the $\mathrm{CD}_{1} 1 \mathrm{~b}^{+}$cells was measured by flow cytometry. Summarized data is shown. Data are represented as mean \pm SEM. $* * * * p<.0001$ 


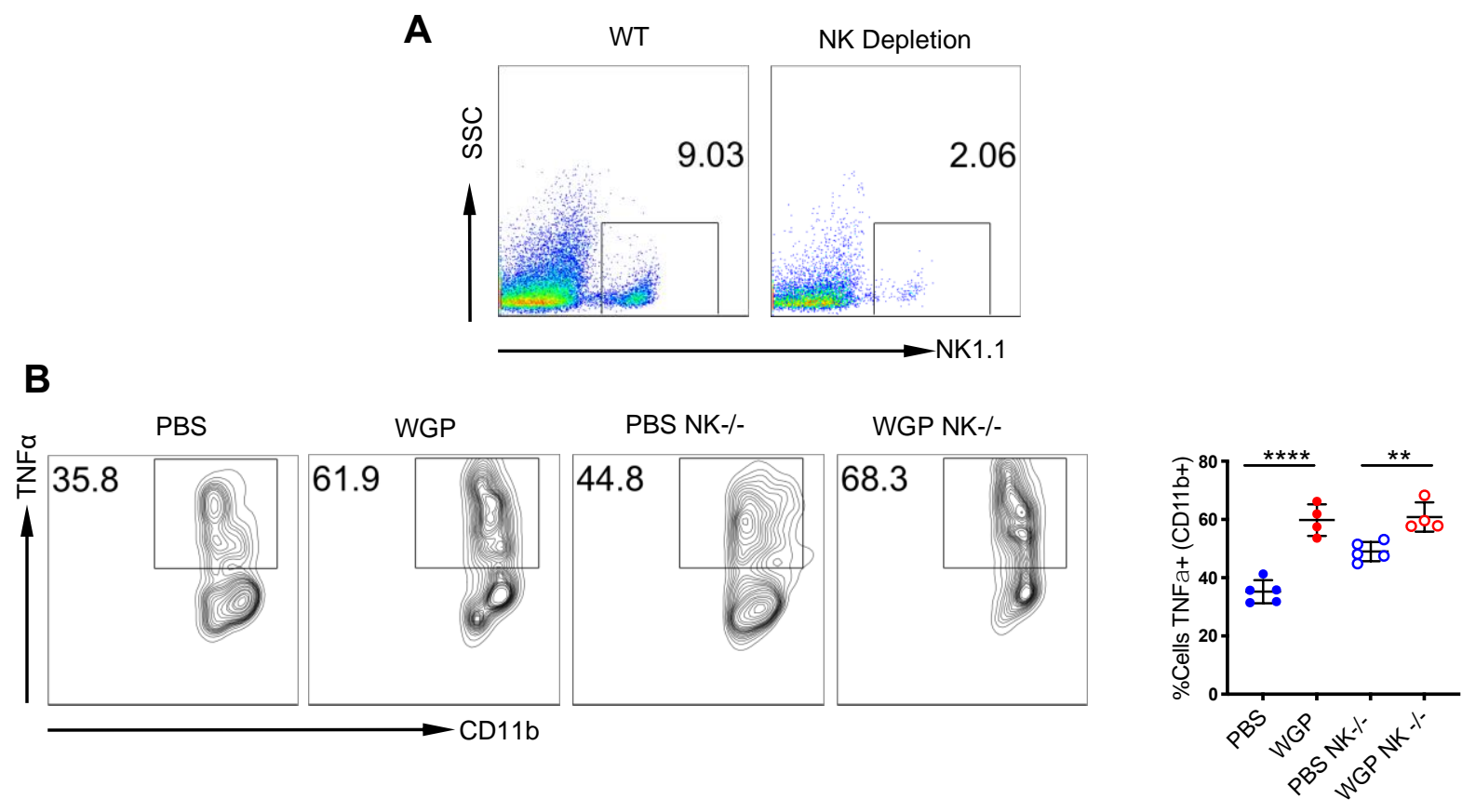

Figure 2.16: Training of pancreatic myeloid cells is not dependent on NK cells $(\mathbf{A}+\mathbf{B})$ NK-cell depleted mice and WT mice were treated with PBS or WGP and 7 days later the pancreatic cells from these mice were restimulated with LPS. TNF $\alpha$ production in the $\mathrm{CD} 11 \mathrm{~b}^{+}$cells was measured by flow cytometry. Summarized data is shown. Data are represented as mean \pm SEM. ns $=$ not-significant; ${ }^{*} p<.05, * * p<.01, * * * p<.001$, $* * * * p<.0001$ 

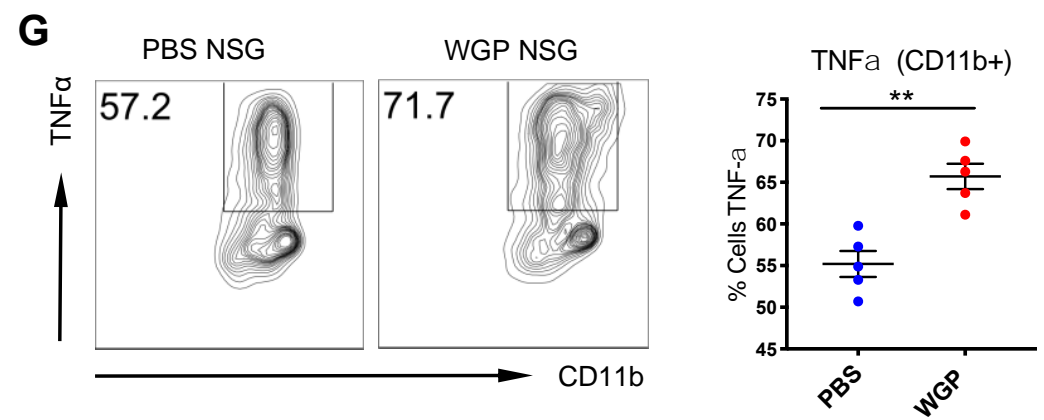

Figure 2.17: Adaptive immune responses to not involved in the training of pancreatic CD11b+ myeloid cells

NSG mice were injected with PBS or WGP and 7 days later pancreatic cells were restimulated with LPS. TNF $\alpha$ production in the $\mathrm{CD}_{11} \mathrm{~b}^{+}$cells was measured by flow cytometry. Representative and summarized data are shown. Data are represented as mean \pm SEM., $* * p<.01$ 
A

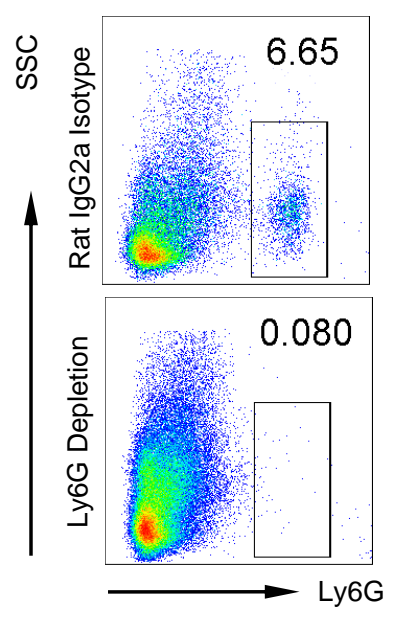

B $P B S+\lg G 2 a \quad W G P+\lg G 2 a$

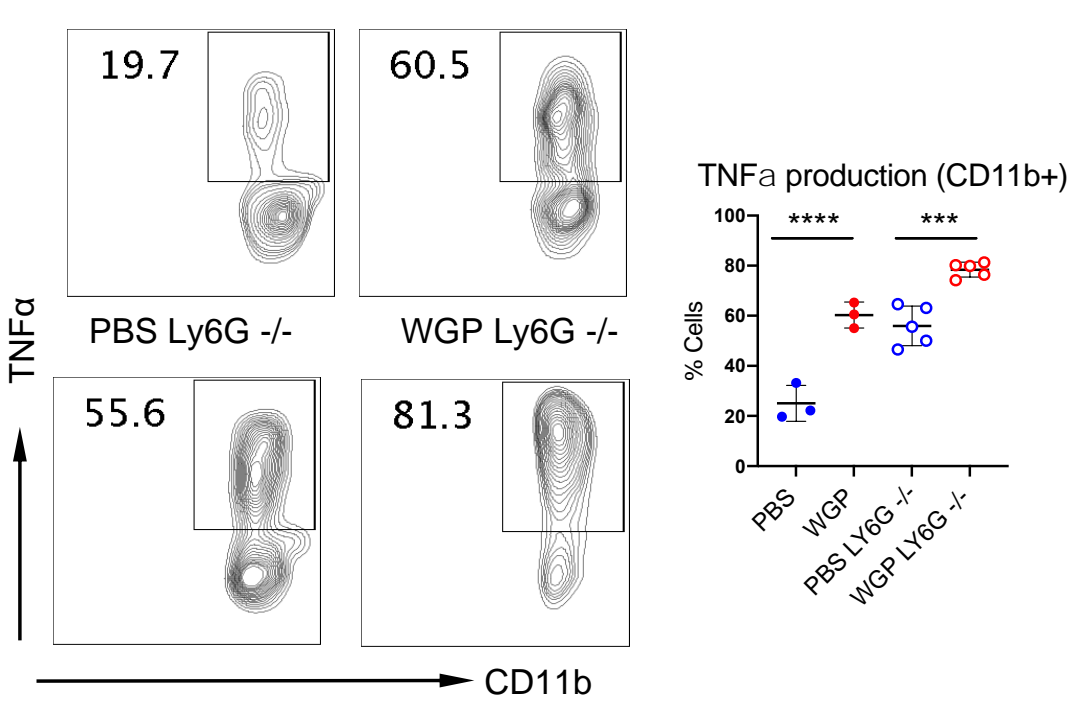

Figure 2.18: Training of pancreatic myeloid cells is not dependent on granulocytes

(A) The depletion efficiency of an anti-ly6G mAb and its isotype control (anti-rat IgG2a)

was assessed in the mouse pancreas. (B) Mice treated with the anti-Ly6G mAb and isotype control were given either PBS or WGP and 7 days later pancreatic cells were restimulated with LPS. TNF $\alpha$ production in the $\mathrm{CD}_{1} 1 \mathrm{~b}^{+}$cells was measured by flow cytometry. Summarized data is shown. Data are represented as mean \pm SEM. ${ }^{* * *} p<.001, * * * * p$ $<.0001$ 
A

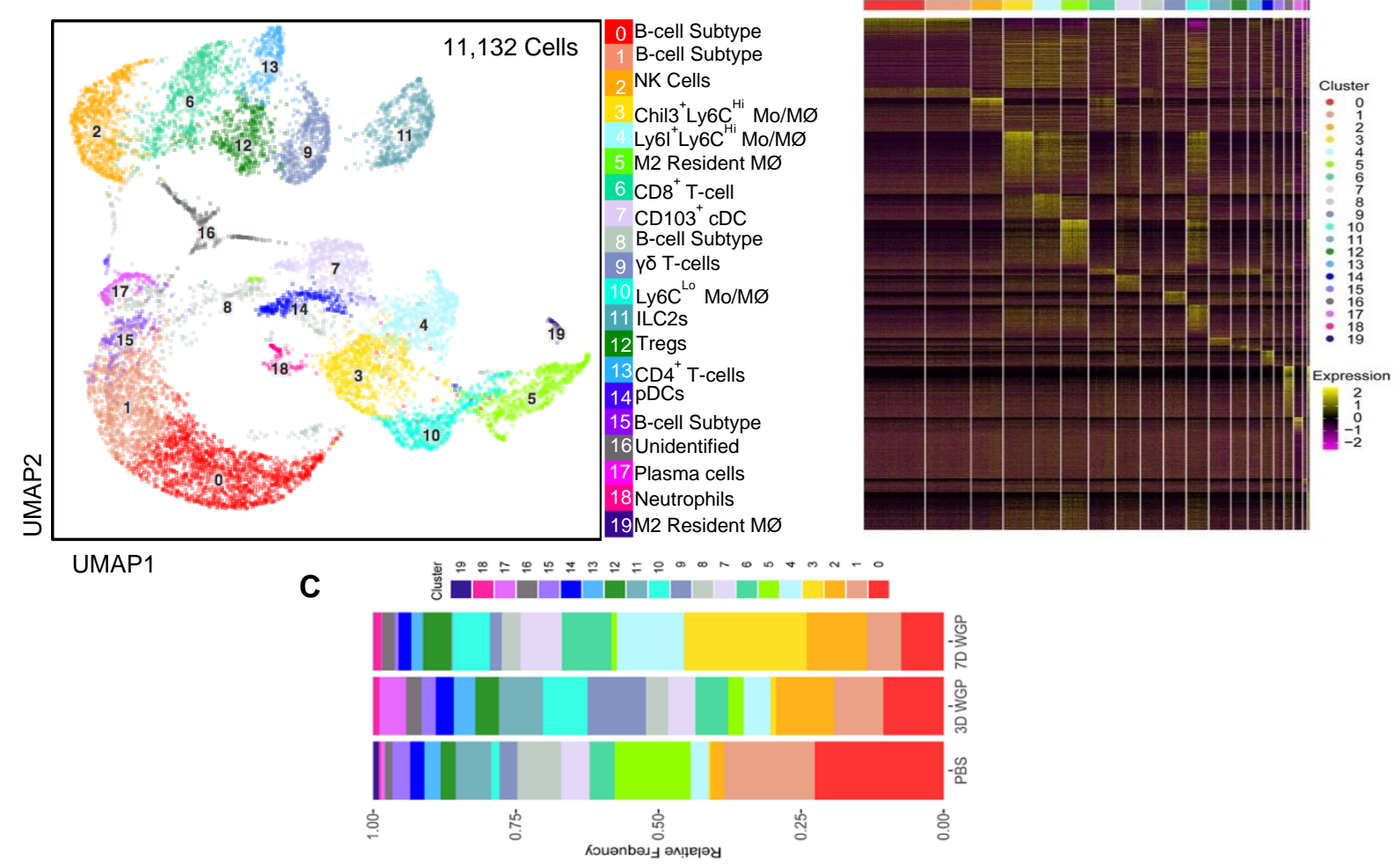

Figure 2.19: Single-cell RNA-Seq showing the immune cell phenotype 3 and 7 days following IP WGP

$\mathrm{CD} 45^{+}$cells from the pancreas of mice treated with PBS or WGP 7 days and 3 days prior were sorted and scRNA-Seq was performed. (A)Two dimensional UMAP representation of 11,132 cells aggregated from the three experimental samples with 20 unique clusters resulting from k-nearest neighbors and Louvain algorithms. (B) Heatmap of expression of aggregated marker genes for all clusters. (C) Bar graphs showing the relative frequency of cells in each cluster across samples. 


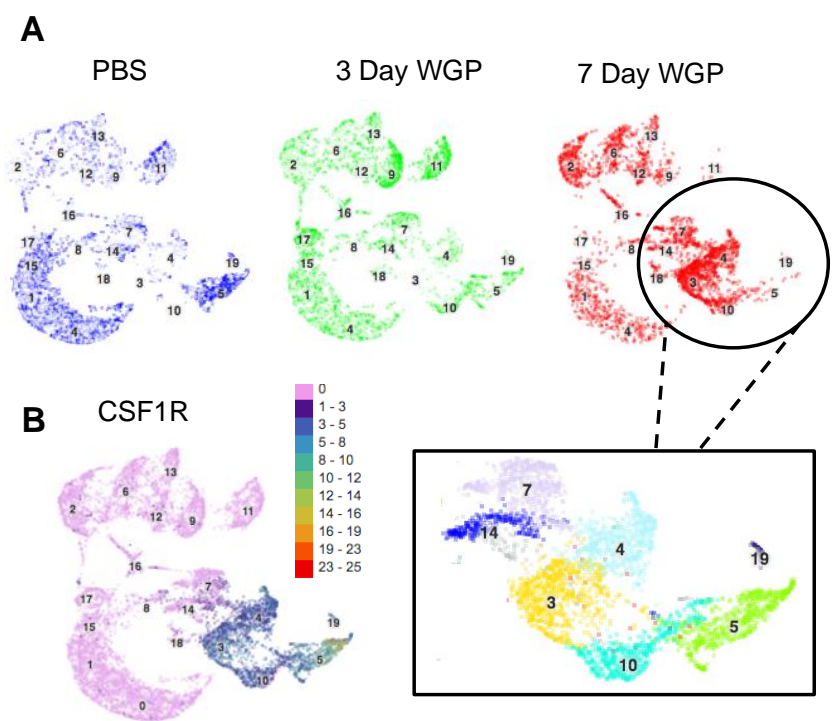

Figure 2.20: scRNA-Seq identifies CSF1R+ myeloid populations that are increased following WGP treatment

$\mathrm{CD} 45^{+}$cells from the pancreas of mice treated with PBS or WGP 7 days and 3 days prior were sorted and scRNA-Seq was performed. (A) UMAP dimension reduction of PBS (blue), 3-day WGP (green) and 7-day WGP (red) samples shown individually. The portion of the UMAP representing myeloid cells is highlighted. (B) Single-cell gene expression of CSFIR. 


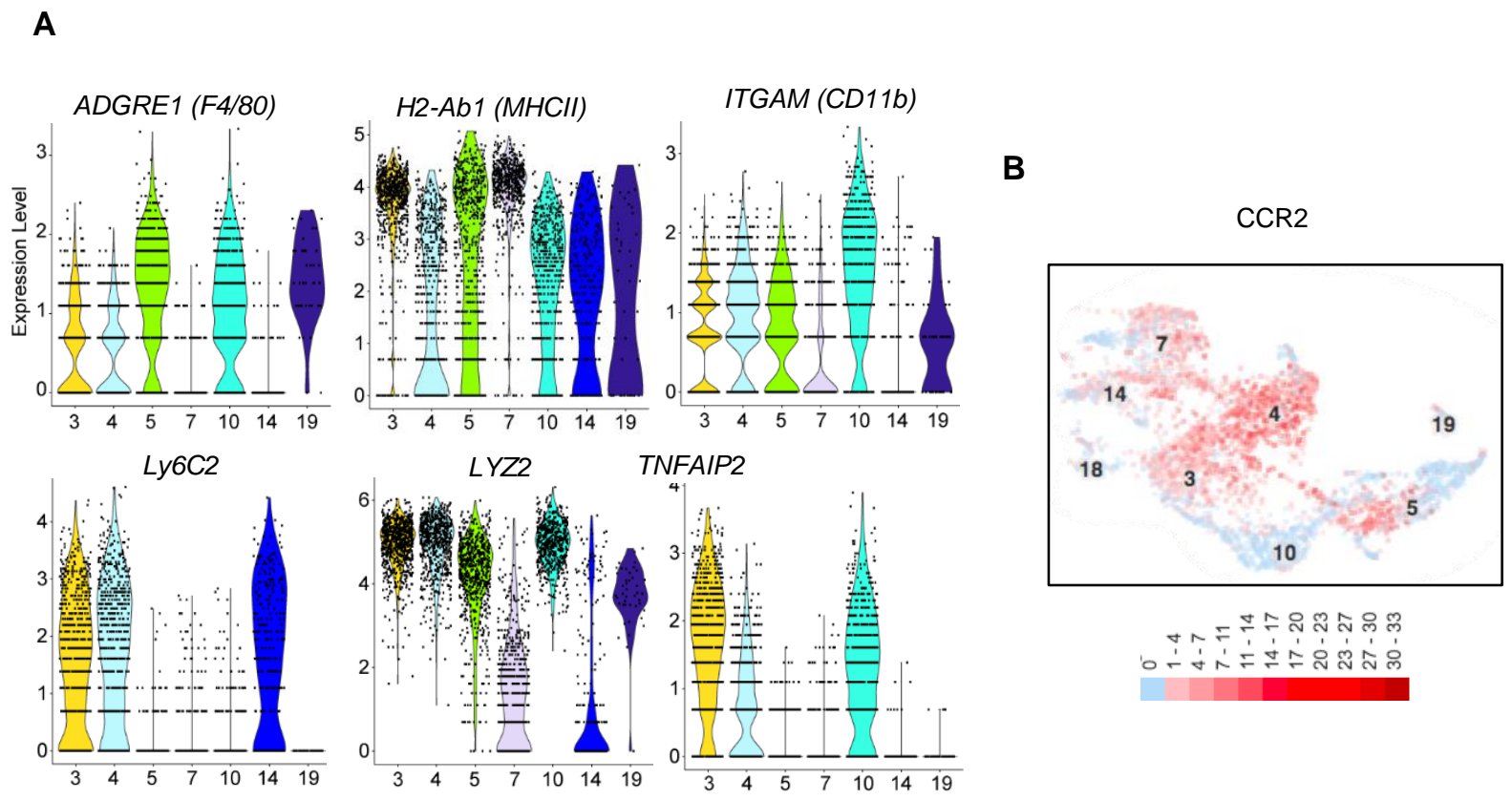

Figure 2.21: Classification of differentially regulated myeloid populations in the pancreas using scRNa-Seq

$\mathrm{CD}_{4} 5^{+}$cells from the pancreas of mice treated with PBS or WGP 7 days and 3 days prior were sorted and scRNA-Seq was performed. (A) Single-cell expression distributions across clusters identified as myeloid cells for select genes related to myeloid phenotyping. (B) CCR2 expression throughout the differentially regulated myeloid population. 

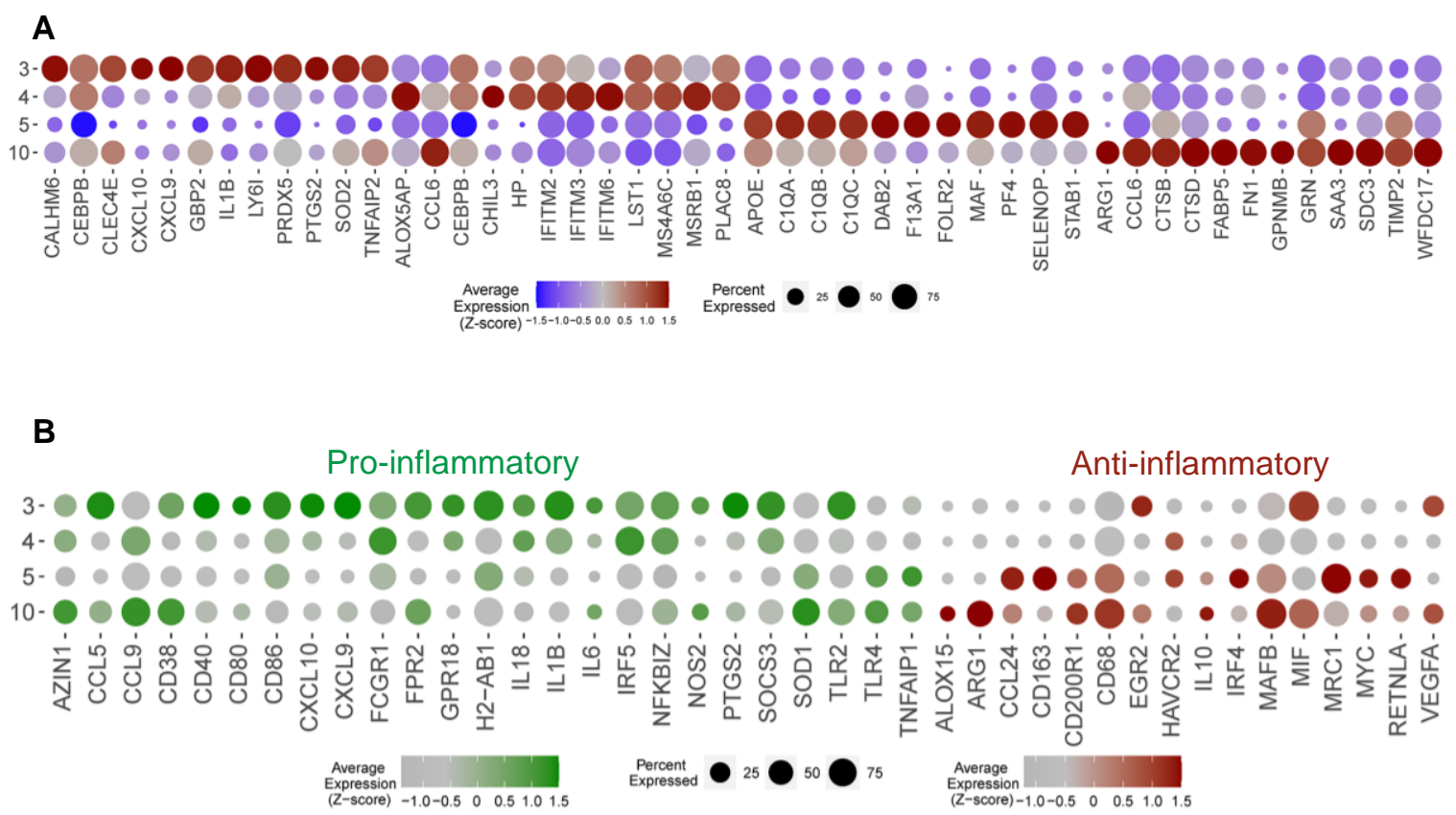

Figure 2.22: Characterization of differentially regulated myeloid populations in the pancreas using scRNA-Seq

$\mathrm{CD}_{4} 5^{+}$cells from the pancreas of mice treated with PBS or WGP 7 days and 3 days prior were sorted and scRNA-Seq was performed. (A) Dot plot of the top 12 enriched genes in cluster 3,4,5 and 10 showing the average expression level and percentage of cells expressing select genes. (B) Dot plots showing the enrichment of selected genes associated with pro-inflammatory (green) and anti-inflammatory (red) immune responses across clusters 3,4,5 and 10. The average expression level is displayed as z-scores computed across the four clusters for individual genes. 


\section{CHAPTER II DISCUSSION}

While it was expected that WGP trafficking to the pancreas would result in immunological changes to the resident populations there, it was surprising to observe such a profound influx of innate immune cells to the pancreas. DTAF-WGP studies demonstrate that while macrophages that have phagocyted WGP traffic to the pancreas, there also exists a population of $\mathrm{CD} 11 \mathrm{~b}+$ myeloid cells that traffic to the pancreas that have not phagocytosed WGP. It is likely that the trafficking of peritoneal macrophages that have phagocytosed WGP to the pancreas incites the production of cytokines and chemokines that then recruit additional myeloid cells there. Despite the presence of these myeloid cells, importantly, both short-term and long-term observation of mice show that this WGP-driven influx of immune cells into the pancreas does not cause morbidity or mortality in mice. Serum amylase levels were not increased, which would be indicative of pancreatic destruction or pathological pancreatitis.

Preliminary $\mathrm{H}+\mathrm{E}$ imaging of WGP-treated pancreases showed an accumulation of immune cells in a fatty layer attached to the pancreas. We hypothesized this to be part of the greater omentum, a fold of visceral peritoneum that attaches to the pancreas in mice. The greater omentum has been known to have immunogenic properties and has also been shown to be a destination of activated peritoneal macrophages[183, 184]. To explore this further we performed omentectomies on mice where we detached the omentum from the

pancreas and removed the distal fold of the tissue. After one week had passed, we injected WGP in an effort to investigate whether the omentum was a critical pathway in WGP- 
trafficking to the pancreas. Despite the lack of a greater omentum, DTAF-WGP still trafficked to the pancreas and CD11b+ myeloid cells still accumulated in the pancreas, though the inflammation caused as a result of the procedure made a full analyses of the pancreas challenging. Kubes et al had reported that in the setting of liver injury, large GATA $^{+}$peritoneal macrophages take an avascular route of migration to the liver where they traffic to and through the visceral endothelium that covers the liver within one hour following the induction of sterile injury[164, 185]. These findings highlight a non-vascular recruitment of immune cells to visceral organs that could be related to the influx of immune cells observed in this model. While chapter 3 of this dissertation will address some of the signaling mechanisms involved in recruiting innate immune cells to the pancreas, it still remains unknown why WGP traffics to the pancreas or why macrophages that have phagocytosed WGP also traffic there.

Following WGP treatment, myeloid cells within the pancreas were shown to exhibit a trained phenotype upon LPS restimulation that was not dependent on any adaptive immune populations. More in depth investigations using CyTOF, RNA-Seq and scRNA-Seq data together confirmed that WGP led to the upregulation in a number of genes associated with trained immunity and enhanced effector function. 4 myeloid populations exhibited noteworthy dynamic behavior following WGP treatment, several of which have not been previously identified in the mouse pancreas. First, cluster 5 which was ITGAM ${ }^{\mathrm{Int}} A D G R E 1^{\mathrm{Hi}} L y z 2^{\mathrm{Hi}} H 2-A b 1^{\mathrm{Hi}} L y 6 C 2^{-}$was present in PBS mice and virtually disappeared following WGP treatment. Their presence in the pancreas of WT mice along with high F4/80 expression, and low Ly6C expression designate cluster 5 as resident macrophages. In line with the literature, these cells strongly exhibited markers of M2 
polarization [186]. Resident macrophages of the pancreas are known to exhibit M2 polarization as these cells are involved in would healing and tissue repair [187]. Cluster 10, defined by the expression of $I T G A M^{\mathrm{Hi}} A D G R E 1^{H i} L y z 2^{\mathrm{Hi}} H 2-A b 1^{\text {Int }} L y 6 C 2^{-}$is absent in PBS treated mice and appears at day 3 after WGP treatment. This population has a similar expression profile to cluster 5 (ITGAM $\left.{ }^{\mathrm{Int}} A D G R E 1^{H i} L y z 2^{\mathrm{Hi}} H 2-A b 1^{\mathrm{Hi}} L y 6 C 2^{-}\right)$, the alternatively activated M2 resident macrophage population, though cluster 10 appears to skew more towards an M1 classically activated macrophage phenotype. The reciprocal increase in cluster 10 and decrease in cluster 5 at days 3 and 7 post-WGP treatment along with a lack of CCR2 expression suggest that cells in cluster 10 could be resident macrophages that have been trained by the $\beta$-glucan, and subsequently adopts an activated phenotype, thus forming a new cluster. While further studies are warranted to investigate whether resident macrophages in the pancreas are impacted by WGP, given the relative disappearance of the resident population following WGP treatment it is likely that this is the case.

Cluster 3 and 4 were both classified as types of inflammatory macrophages/monocytes based on the expression of $I T G A M^{\mathrm{Int}} A D G R E 1^{\text {Int }} L y z 2^{\mathrm{Hi}} H 2-A b 1^{\text {Int }} L y 6 C 2^{\mathrm{Hi}}$ along with high expression of TNFAIP2 and CCR2. Cluster 3, a novel pancreatic population which exhibits the most evident features of trained immunity is absent in naïve mice and becomes the cluster with the highest relative frequency by day 7 after WGP administration. Recent studies into the mechanism of trained immunity show that after $\beta$-glucan administration, monocytes from trained HSCs in the bone marrow can migrate to peripheral organs where they become monocyte-derived macrophages that not only are longer lived than typical monocytes, but that also possess features of trained immunity [188, 189]. Given the trained 
phenotype, the high expression of CCR2, and that the majority of the influx of this population happens more than 3 days following WGP administration, we speculate that cluster 3 and 4 may represent such BM-derived cells that are directed to the pancreas shortly after WGP arrives there. This development of peripheral trained immunity has been described in several tissues but has not been previously identified to exist in the pancreas $[190,191]$. 


\section{CHAPTER III}

\section{WGP-INDUCED TRAFFICKING OF IMMUNE CELLS TO THE PANCREAS IS CCR2 DEPENDENT}

\section{CHAPTER III INTRODUCTION}

After discovering that the two most prominent cell populations to traffic to the pancreas due to WGP treatment were macrophage/monocyte populations that highly expressed CCR2, we wanted to know whether CCR2 signaling may be responsible for this influx of cells into the pancreas. CCR2 is known to be highly expressed on blood monocytes, and CCR2-/- mice are known to have increased numbers of monocytes in the BM, suggesting that CCR2 expression is critical to monocyte egress from the BM [192]. Monocyte chemoattractant protein-1 (MCP-1), also known as CCL2, is the primary ligand for CCR2, and is expressed by a variety of cell types including endothelial cells, smooth muscle, fibroblasts [193, 194], tumor cells [195] and myeloid cells. CCL2 is best known as a chemoattractant, where its expression induces monocyte adhesion to the vascular endothelium, extravasation through blood vessels and chemotaxis to tissues with high CCL2 concentrations [196]. Overall, CCL2 expression is critical in both recruiting circulating monocytes from the periphery to a site of inflammation, and in mobilizing CCR2+ monocytes from the bone marrow [192]. Given the observed recruitment of monocytes and monocyte-derived macrophages to the pancreas following WGP injection, 
we hypothesized that CCR2/CCL2 signaling may be important in the recruitment of these cells.

\section{CHAPTER III RESULTS}

To investigate the mechanism of myeloid cell recruitment to the pancreas, RNA-Seq data was used to characterize chemokines and cytokines whose expression was significantly upregulated upon WGP treatment (Fig 3.1A), and scRNA-seq data was used to investigate which cells might be responsible for this upregulation (Fig 3.1B). While several chemokines and cytokines were upregulated, our observation of macrophage and monocyte influx into the pancreas piqued a specific interest in CCR2 due to its involvement in monocyte and macrophage recruitment and in monocyte egress from the bone marrow [197-199]. CyTOF data also showed a prominent increase in CCR2 positive cells after WGP treatment (Fig 3.2A) and scRNA-Seq showed a distinct expression of CCR2 in clusters 3 and 4, which were the two populations that showed the most distinct phenotypes of trained immunity (Fig 3.1B). Additionally, 24 hours post-WGP treatment, whole pancreatic lysates showed a 30-fold increase in CCL2, which is the ligand for CCR2 and is involved in mediating monocyte chemotaxis (Fig3.2B)

While RNA-Seq data had shown a clear signature of immune cell recruitment and trafficking, these data had also indicated that WGP upregulated proliferation of leukocytes and mononuclear cells (Fig 3.3A). To investigate whether the CCR2 ${ }^{+}$myeloid cells were proliferating once they reached the pancreas, WT mice were treated with WGP or PBS and 7 days later pancreatic cells were assessed for Ki67 expression. Cells were first gated on the $\mathrm{CD} 45^{+} \mathrm{Ki} 67^{+}$population, and these were then gated on $\mathrm{CD} 11 \mathrm{~b}$ vs $\mathrm{CCR} 2$ to determine 
what percent of the proliferating cells were $\mathrm{CCR} 2^{+}$myeloid cells. Following WGP treatment there was an increase in overall proliferating cells (Fig 3.3B) and a large percent of these proliferating cells were $\mathrm{CD} 11 \mathrm{~b}^{+} \mathrm{CCR} 2^{+}$(Fig 3.3C). We then investigated the contribution of $\mathrm{CCR} 2^{+}$cells to the trained phenotype. 7 days after in vivo treatment with PBS or WGP, $\mathrm{CCR} 2^{+}$and $\mathrm{CCR} 2^{-}$populations were measured for trained response (Fig 3.4). This data indicated that the majority of cells trained following WGP treatment were $\mathrm{CCR} 2{ }^{+}$. To further examine the role of CCR 2 in $\beta$-glucan trained monocytes/macrophages, $\mathrm{CCR} 2^{-/-}$mice were trained with WGP $\beta$-glucan. CCR2 $2^{-/-}$mice did not undergo an influx of $\mathrm{CD}^{+} 5^{+}$(Fig 3.5A) or $\mathrm{CD} 45^{+} \mathrm{CD} 11 \mathrm{~b}^{+}$myeloid cells (Fig 3.5B) into the pancreas, and did not show a trained response as revealed by TNF- $\alpha$ production (Fig 3.5C). This data indicates that CCR2 plays a critical role in the migration of innate immune cells to the pancreas and in the induction of peripheral trained immunity in the pancreas.

As previously noted, CCR2 is important for early recruitment of monocytes, but less so for late recruitment [200]. Thus far, we had also observed that at 7 days following WGP training, CCR2 was critical to the recruitment of trained myeloid cells to the pancreas. We next examined whether this influx occurred at an early time point upon WGP training and whether the $\mathrm{CCR} 2^{+}$myeloid cells were recruited to the pancreas. To this end, mice were treated with PBS or WGP and pancreatic tissues were analyzed 24 and 48 hours later. Surprisingly, as early as 24 hours after WGP training, increases in $\mathrm{CD}^{+} 5^{+}$(Fig 3.6A), $\mathrm{CD} 11 \mathrm{~b}^{+}$(Fig 3.6B), F4/80 ${ }^{+}$(Fig 3.6C), $\mathrm{Ly} \mathrm{C}^{+}$(Fig 3.6D) and CD11b $\mathrm{b}^{+} \mathrm{CCR} 2^{+}$(Fig 3.6E) cells were observed. Additionally, it was shown that these myeloid cells were also trained as early as 24 hours following WGP treatment. This observation highlights a divergence 
between these results and previously observed phenotypes of trained immunity which usually are not initiated until at least 3 days following training (Fig 3.6F).

\section{CHAPTER III FIGURES}

A

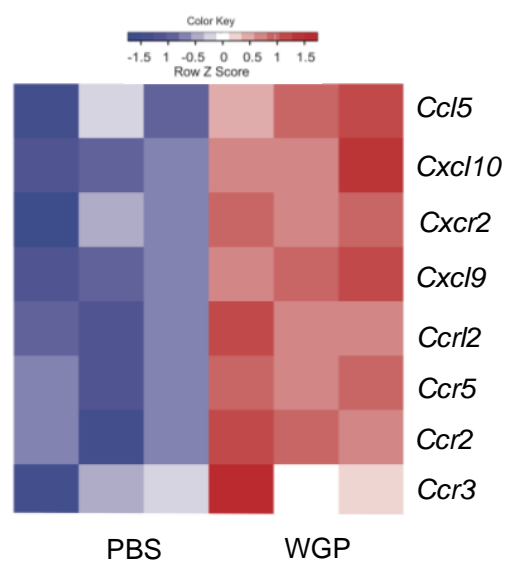

B

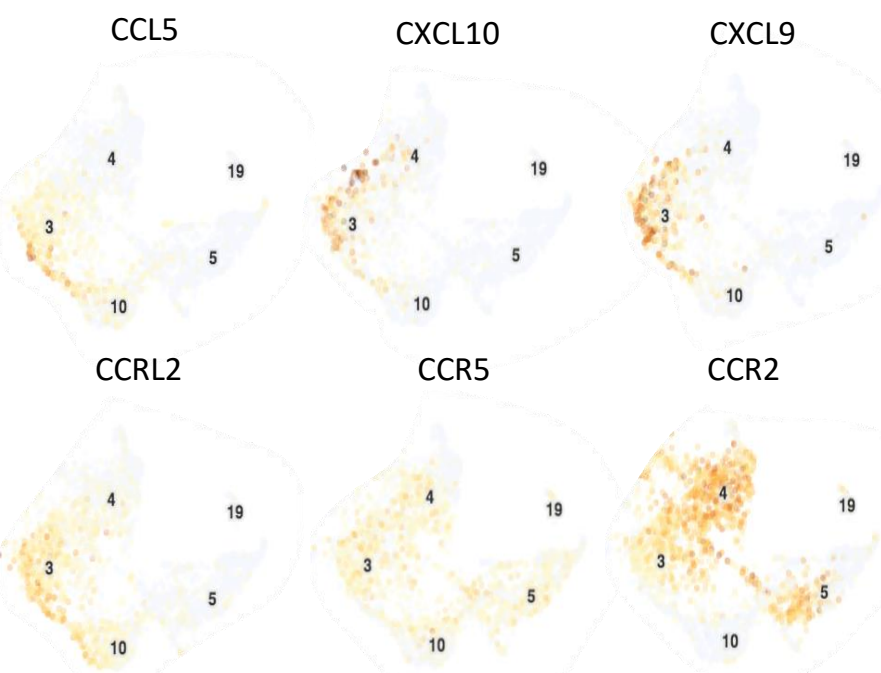

\section{Figure 3.1: Chemokines and cytokines upregulated by WGP treatment}

(A) Heatmap of chemokines and cytokines that were upregulated in 7-day WGP treated

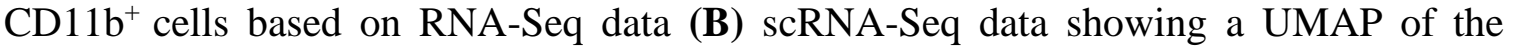
myeloid cluster expression of chemokines and chemokine receptors. 
A

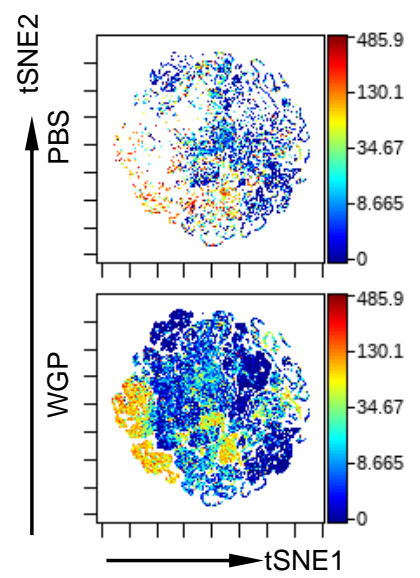

B

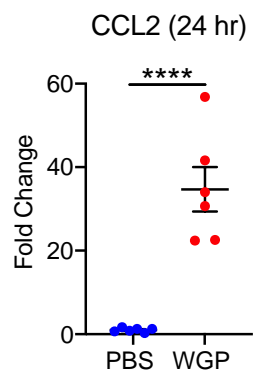

Figure 3.2: CCR2/CCL2 signaling is increased due to WGP treatment

(A) viSNE plot of the CD11 $\mathrm{b}^{+}$pancreatic population in PBS and 7-day WGP-trained mice, highlighting the expression of CCR2. Images made with CyTOF data. (B) CCL2 expression in whole pancreatic lysates 24 hours following WGP treatment a measured by RT-PCR. 

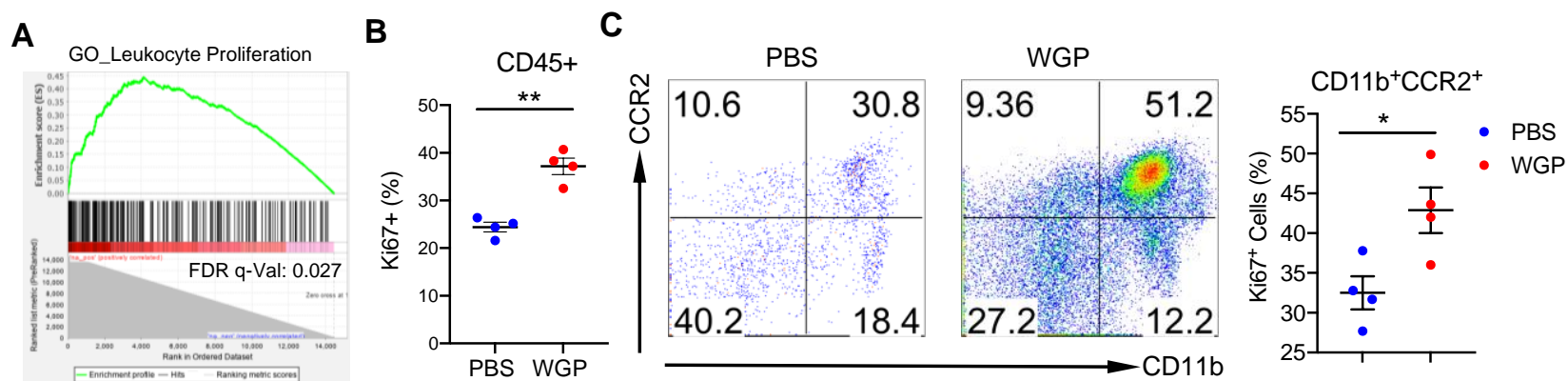

Figure 3.3: Proliferation of CCR2+ cells in the pancreas

(A) GSEA generated enrichment plots of genes related to leukocyte proliferation in

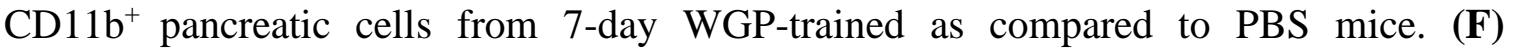
Summarized data of the percent of $\mathrm{CD} 45^{+}$pancreatic cells that are Ki67+ in PBS and 7day WGP-trained mice. (B) Cells were first gated on the CD45+Ki67+ population. Plots show the percent of the $\mathrm{CD} 45^{+} \mathrm{Ki} 67^{+}$proliferating pancreatic cells that are $\mathrm{CD} 11 \mathrm{~b}^{+} \mathrm{CCR} 2^{+}$ in PBS and 7-day WGP-trained mice. Data are represented as mean \pm SEM. ${ }^{*} p<.05, * * p$ $<.01$. 

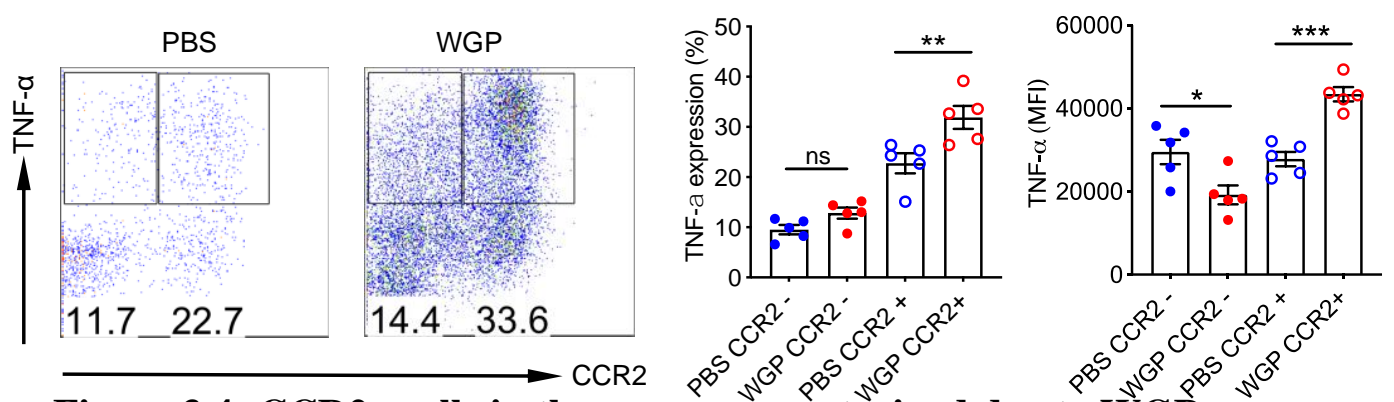

Figure 3.4: CCR2+ cells in the pancreas are trained due to WGP

Pancreatic cells from PBS and 7-day WGP-trained mice were restimulated with LPS and the percent of CCR2 positive and negative cells producing TNF $\alpha$ was measured in each condition. Cells were first gated on the $\mathrm{CD} 45^{+} \mathrm{CD} 11 \mathrm{~b}^{+}$subset. Data are represented as mean \pm SEM. ns= not significant; $* * p<.01, * * * p<.001$ 

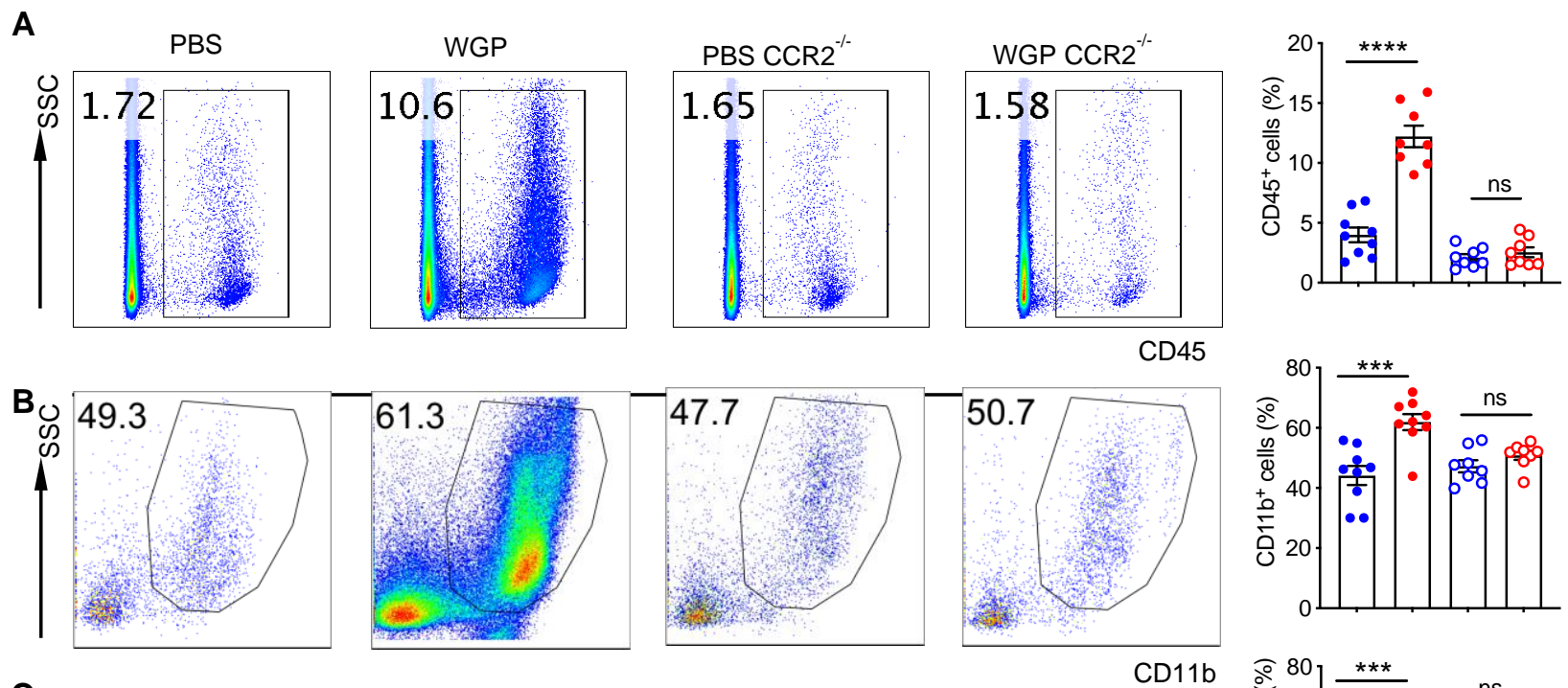

C
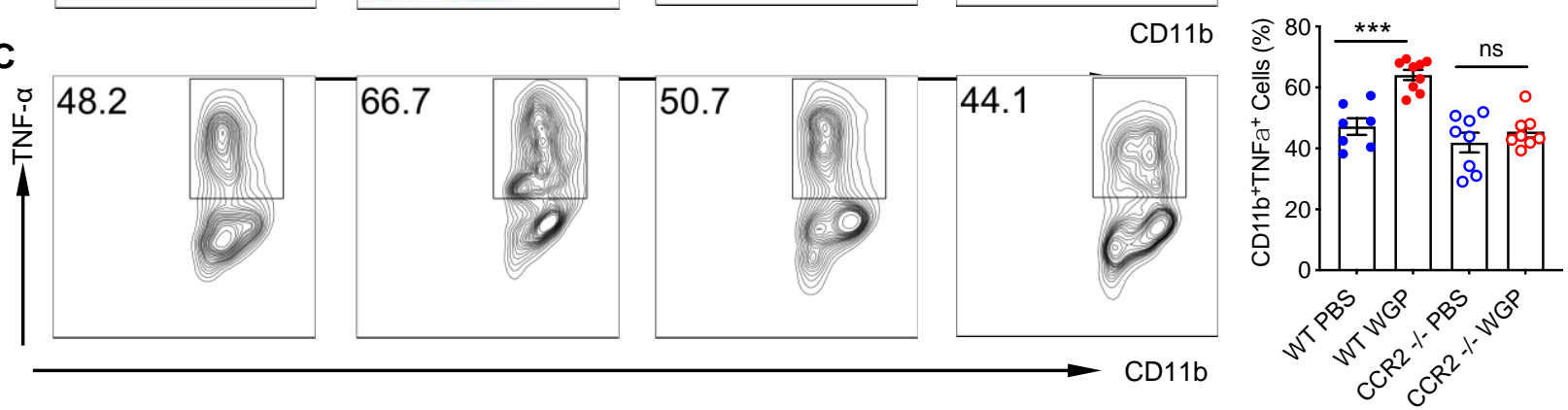

Figure 3.5: cellular influx and training of pancreatic innate myeloid cells do not occur in CCR2-/- mice

(A-C) WT and CCR2 $2^{-/-}$mice were treated with PBS or WGP and the percent of (A) CD45 cells in the pancreas (B) and the percent of $\mathrm{CD}^{4} 5^{+}$cells that were $\mathrm{CD} 11 \mathrm{~b}^{+}$was assessed. (C) The percent of $\mathrm{CD} 45^{+} \mathrm{CD} 11 \mathrm{~b}^{+}$cells producing $\mathrm{TNF} \alpha$. Data are represented as mean \pm SEM. ns= not significant; $* * * p<.001$, 
A

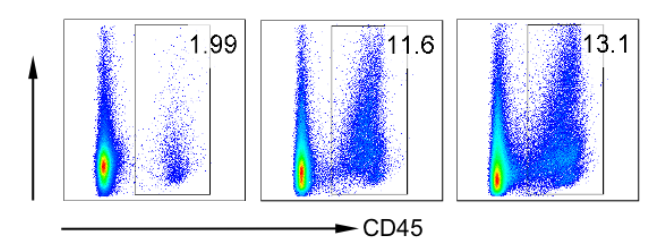

B

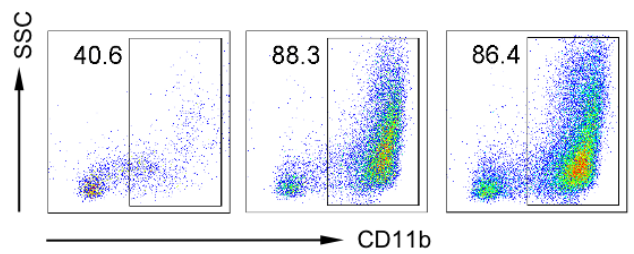

C

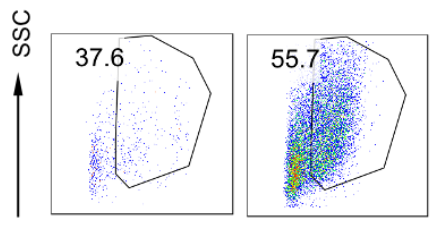

D

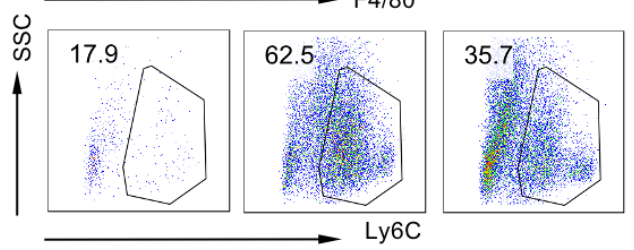

E

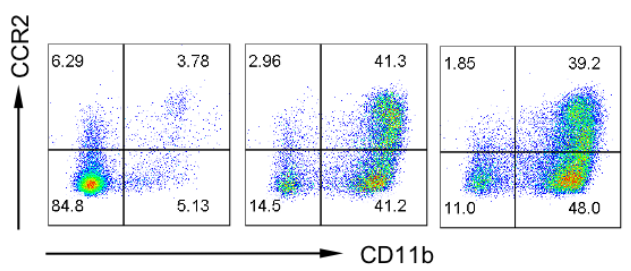

F
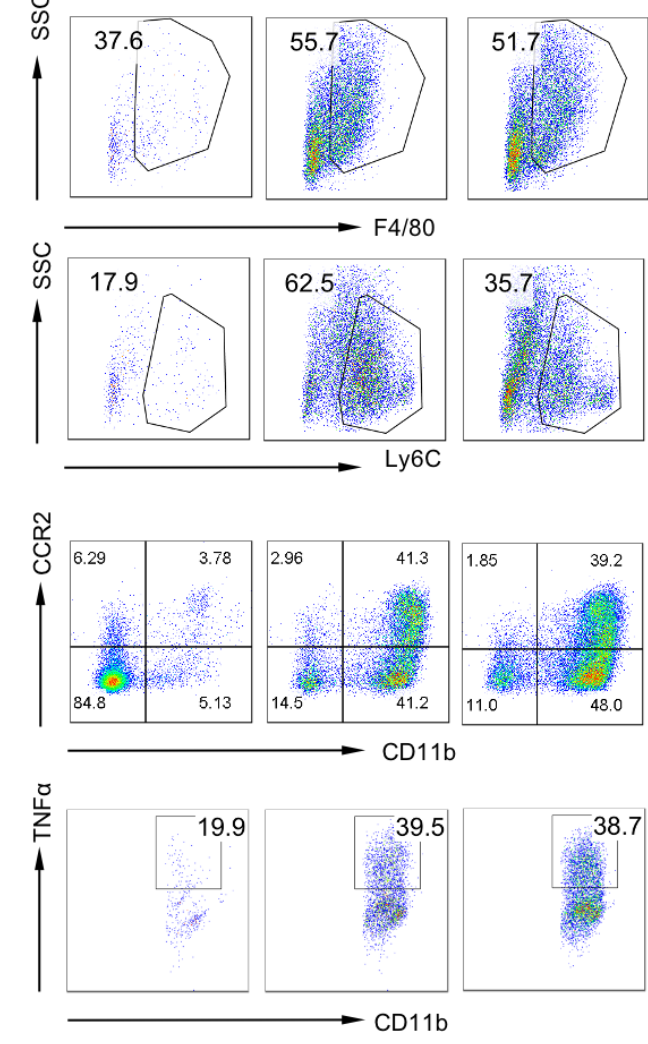

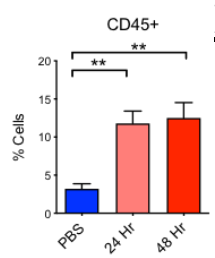

Figure 3.6: Pancreatic myeloid cells are trained by 24 and 48 hours postWGP

Mice were treated with WGP 24 hours

or 48 hours before euthanization and the

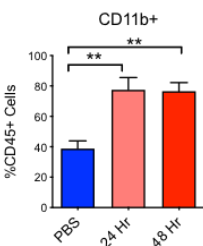

percent of (A) $\mathrm{CD}^{+} 5^{+}$, (B) percent of $\mathrm{CD}^{2} 5^{+}$cells that were $\mathrm{CD} 11 \mathrm{~b}^{+},(\mathbf{C})$ percent of $\mathrm{CD} 45^{+} \mathrm{CD} 11 \mathrm{~b}^{+}$cells that were

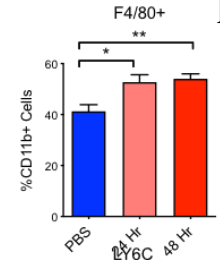

$\mathrm{F} 4 / 80^{+}$and (D) the percent of $\mathrm{CD} 45^{+} \mathrm{CD} 11 \mathrm{~b}^{+}$cells that were $\mathrm{Ly} 6 \mathrm{C}^{+}$

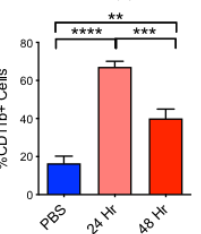

cells were measured and compared to that of a PBS treated mouse. (E) Pancreatic cells from PBS and 24 and

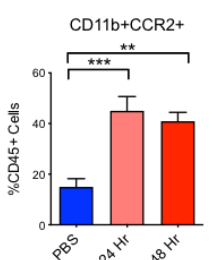

48 hour WGP treated mice were gated on the CD45+ population and the percent of these cells that were $\mathrm{TNF}_{\mathrm{N}} \mathrm{CD} 11 \mathrm{~b}+$

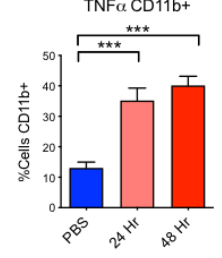

$\mathrm{CD}_{11 \mathrm{~b}^{+} \mathrm{CCR} 2^{+}}$was assessed. (F)

Pancreatic cells from PBS and 24 and 48 hour WGP treated mice were

restimulated with LPS and the production of TNF $\alpha$ was measured. All analyses were done by flow cytometry and representative and summarized data are shown. Data are represented as mean \pm SEM. $\mathrm{ns}=$ non-significant; ${ }^{*} p<.05, * * p<.01, * * * p<.001, * * * * p<.0001$ 


\section{CHAPTER III DISCUSSION}

24 hours following the administration of WGP, CCL2 levels in whole pancreatic lysates were found to be increased by 30 fold. Accordingly, we show that the recruitment of immune cells and the establishment of this peripheral trained immunity in the pancreas is dependent on CCR2 signaling. We also show that CCR2+ cells account for the majority of cells that are trained following WGP treatment. Together this suggests that WGP trafficking to the pancreas upregulates CCL2 signaling which then is sufficient to recruit $\mathrm{CCR}^{+}$trained cells from the bone marrow to the pancreas. Though peripheral trained immunity has been documented, the specific signaling mechanism responsible for the recruitment of trained innate cells from the bone marrow to a specific organ to establish peripheral trained immunity has not been well defined. We speculate that cluster 3 and 4, from the scRNA-seq data, may likely represent such BM-derived cells that are directed to the pancreas due to the CCL2 signaling that occurs shortly after WGP arrives there. These subsets of cells are only present more than 3 days following WGP treatment and show high levels of CCR2, an M1 polarization phenotype and gene upregulations consistent with the induction of trained immunity.

As after WGP treatment we had observed a disappearance of the resident macrophage population, likely due to a phenotypic shift, we did wonder whether resident macrophages may be involved in the initiation of CCL2 production that recruits CCR2+ cells to the pancreas. To address this we tried to deplete resident pancreatic macrophage populations, however this proved to be challenging. The use of both liposomal clodronate and antiF4/80 mAb depleted macrophages systemically, such as in the spleen, but was not effective at depleting the pancreatic resident population. Further experiments are needed to 
determine if the peritoneal macrophages that phagocytose WGP, resident macrophages or other cell types in the pancreas are responsible for initiating CCL2 signaling. CCL2 is also highly produced by stromal cells, so it may be the case that resident macrophage populations do not directly secrete CCL2, but rather impact other cell types within the pancreas to produce CCL2.

We also showed that CCR2 ${ }^{+}$cells account for a large percent of the proliferating $\mathrm{Ki}_{6} 7^{+}$ cells in the pancreas following WGP training. Taken together these results suggest that the increase in trained myeloid cells may be due to several mechanisms including the influx of peritoneal macrophages, trafficking of $\mathrm{CCR} 2^{+}$cells to the pancreas from the periphery, and through in situ expansion of trained resident macrophages. Notably, the currently reported study is the first time that CCR2 has been identified as a requisite to the establishment of organ-specific peripheral trained immunity. 


\section{CHAPTER IV}

\section{THE ANTI-TUMOR ROLE OF $\beta$-GLUCAN-INDUCED TRIANED IMMUNITY IN PANCREATIC CANCER}

\section{CHAPTER IV INTRODUCTION}

Our investigations up to this point have shown that WGP treatment incites an influx of myeloid cells into the pancreas that show a phenotype of trained immunity and M1 polarization. Not only this, but WGP trafficking to the pancreas was also shown to reprogram the M2 resident macrophages of the pancreas into a more inflammatory phenotype. Moreover, these studies revealed in both in vitro and in vivo settings that pancreatic CD11b+ cells that had been exposed to WGP produced increased antiinflammatory cytokines, such as TNF- $\alpha$ and IL-6, upon re-exposure to a secondary stimulus, LPS. It is known that there are a plethora of stimuli that can serve as the second signal in $\beta$-glucan-induced trained immunity. However, the majority of studies have used bacteria or viruses as a secondary stimulus, and thus have been mainly been targeted at understanding whether the induction of trained immunity may be useful against pathogenic infections. Further, while the two studies regarding trained immunity and cancer discussed in the introduction do investigate whether the induction of trained immunity in mice can lead to anti-tumor effects, they do not directly investigate if the trained response can be

directly elicited by exposure of trained cells to a tumor cell or the soluble factors that tumor cells secrete. We hypothesized that tumor cells and their soluble factors may function as 
an effective second signal to cells previously trained by $\beta$-glucan (Fig 4.1). Additionally, given the influx of trained immune cells that we had observed, in this chapter we will explore the anti-tumor mechanisms of trained immunity specifically in a model of orthotopic pancreatic cancer.

\section{CHAPTER IV RESULTS}

\section{WGP-trained pancreatic infiltrating myeloid cells elicit potent trained responses to}

\section{factors secreted from pancreatic cancers and exhibit enhanced phagocytosis and}

\section{ROS-mediated cytotoxicity}

As we have shown that using WGP as an initial stimulus results in innate immune cells that are trained to respond more robustly to a secondary exposure of LPS, and that these cells accumulate in the pancreas following treatment, we wanted to know whether secondary stimuli related to pancreatic tumors may also elicit this trained response. To this

end, we first investigated whether pancreatic cancer cells themselves are capable of eliciting the WGP-induced trained response. To probe this question, peritoneal macrophages were cultured with PBS or WGP in vitro for 24 hours and then washed, followed by a 7-day resting period. On day 7, cells were restimulated with LPS, the supernatant from cells cultured from a naïve mouse pancreas, and the supernatant from cultured KPC cells, which are a cell line of a pancreatic tumor on a C57BL/6 background derived from the $L S L-K r a S^{G 12 D /+} ; L S L-T r p 53^{R 172 H /+}$; Pdxl-Cre (KPC) or Pan02 pancreatic cancer cells for 24 hours. TNF- $\alpha$ production in the supernatant was measured by ELISA. Compared to the supernatant from cultured normal pancreatic cells which did not activate 
previously trained macrophages, the supernatant from cultured KPC and Pan02 pancreatic cancer cells stimulated more TNF- $\alpha$ production in $\beta$-glucan trained macrophages (Fig 4.2A). To control for the possibility that the process of phagocytosis itself may cause cells to become activated, peritoneal macrophages were cultured with $3 \mu \mathrm{m}$ polystyrene microparticle beads, the cells were allowed to rest for 7 days, and cells then were restimulated with PBS or LPS (Fig 4.2B). Results showed that enhanced production of TNF $\alpha$ was specific to WGP training.

This was then tested in an ex vivo setting in which mice were treated with PBS or WGP and 7 days later pancreatic myeloid cells were harvested and restimulated with the supernatants from cultured KPC (Fig 4.3A) or Pan02 cells (Fig 4.3B). It showed that WGP in vivo trained $\mathrm{CD} 11 \mathrm{~b}^{+}$myeloid cells in the pancreas produced significantly more TNF- $\alpha$ in response to tumor-conditioned media.

We had shown that tumor cell supernatants function as secondary stimulus to trained cells, however there are a multitude of factors within the tumor conditioned media that may specifically function as the second stimulus in trained immunity. It is known that pancreatic tumor cells express high levels of damage-associated molecular patterns (DAMPs) and pro-inflammatory factors, and we hypothesized that some of these specific factors may evoke the trained response. KPC cells are known to secrete high levels of both macrophage migration inhibitory factor (MIF) and high mobility box protein-1 (HMGB1). MIF is a cytokine that is known to be secreted in high concentrations by pancreatic tumors that can act directly on myeloid cells [201, 202]. Indeed, MIF was present in the supernatant of KPC and Pan02 cells as assessed by ELISA (Fig 4.4A). We thus hypothesized that MIF might be a potential tumor-secreted factor that has the capacity to act as a second signal in 
the setting of WGP-induced trained immunity. To investigate this, pancreatic myeloid cells from in vivo WGP-trained mice were restimulated with a similar concentration of recombinant MIF (rMIF) as present in tumor conditioned media. As shown in Fig 4.4B, pancreatic myeloid cells previously trained with WGP showed enhanced TNF- $\alpha$ production upon rMIF restimulation. Re-stimulating WGP-trained cells with HMGB1 also was shown to elicit the trained response (Fig 4.5). Collectively, these data suggest the novel concept that pancreatic tumor cells, through soluble factors that they release, can serve as the second signal to activate myeloid cells in the pancreas that have been trained by WGP.

We next examined whether these WGP-trained innate immune cells have enhanced intrinsic anti-tumor properties. RNA-Sequencing data indicated that phagocytosis-related mechanisms were upregulated in the WGP setting, which we hypothesized could be one mechanism of anti-tumor functionality (Fig 4.6). $\mathrm{CD}^{+} 5^{+}$pan immune cells and CD11b myeloid cells from WGP-trained mouse pancreas were harvested and assayed for phagocytotic potential using the Invitrogen pHrodo $^{\mathrm{TM}}$ Green S. aureus BioParticles ${ }^{\mathrm{TM}}$ Phagocytosis Kit. WGP treatment led to a significant increase in the phagocytic potential of overall $\mathrm{CD}^{+} 5^{+}$immune cells (Fig 4.7A) and in CD11b $\mathrm{b}^{+}$myeloid cells (Fig 4.7B). In addition, in vivo trained myeloid cells showed an increase in the phagocytosis of KPC tumor cells (Fig 4.8). We then assessed whether myeloid cells trained by WGP show an increased cytotoxicity to KPC cells. RNA-Seq data indicated that DEGs related to reactive oxygen species (ROS) biosynthetic processes and positive regulation of ROS metabolic processes were significantly enriched in WGP-treated myeloid cells (Fig 4.9A). As a result, we hypothesized that the upregulation of ROS production by WGP would result in increased pancreatic myeloid cell cytotoxicity to KPC tumor cells. To explore this 
hypothesis, $\mathrm{CD} 11 \mathrm{~b}^{+}$cells from the pancreas were isolated from $\beta$-glucan trained or untrained mice. Acquiring enough $\mathrm{CD}_{1} 1 \mathrm{~b}^{+}$cells from an untrained mouse pancreas was challenging, as only $1 \%$ of total cells are $\mathrm{CD} 45^{+}$in pancreas. As such, our assay was limited to a maximum of 1:20 for the tumor cell:CD11 $\mathrm{b}^{+}$cell ratio. $\mathrm{CD} 11 \mathrm{~b}^{+}$cells were plated with luciferase expressing KPC cells $\left(\mathrm{KPC}^{\mathrm{Luc}}\right)$ for 24 hours. The luciferase released into the supernatant was quantified using the Luciferase Assay Reagent. WGP-trained myeloid cells showed a 3-fold increased cytotoxicity towards KPC tumor cells, and the inhibition of ROS production using the ROS inhibitor N-Acetyl Cysteine (NAC) completely abrogated the WGP-elicited increase in cytotoxicity (Fig 4.9B). Ultimately, these data identifies that pancreatic tumor cells are capable of reactivating WGP-trained infiltrating myeloid cells in the pancreas, and that these cells show enhanced phagocytosis and ROSmediated cytotoxicity.

\section{WGP-induced trained immunity reduces tumor growth and prolongs survival in orthotopic models of pancreatic cancer}

We next investigated whether $\beta$-glucan-mediated trained innate immune responses in the pancreas would result in the establishment of an anti-tumor microenvironment that may be sufficient to overcome the characteristically immunosuppressive TME of PDAC. Accordingly, mice were given 1 dose of either PBS or WGP on day -7 and on day $0,1 \times 10^{5}$ KPC or KPC ${ }^{\text {Luct }}$ cells were orthotopically implanted into the tail of the pancreas (Fig 4.10A). At day 21 mice were euthanized and the tumor weight was measured or (Fig 4.10B). In the setting of injection of $\mathrm{KPC}^{\mathrm{Luct}}$ cells, mice were injected with luciferase substrate and tumors were imaged. The tumor burden was assessed through quantification 
of photon counts/second (Fig 4.10C). Both studies showed a remarkable reduction in tumor burden as a result of WGP treatment, and survival was also significantly prolonged in mice trained with WGP (Fig 4.10D). Immunophenotyping of the tumors showed a persistent increase in $\mathrm{CD}_{4} 5^{+}$immune cells, $\mathrm{CD} 11 \mathrm{~b}^{+}$myeloid cells and $\mathrm{F} 4 / 80^{+}$macrophages in the WGP-trained setting (Fig 4.11A). CD11 $\mathrm{b}^{+}$myeloid cells (Fig 4.11B) and CD11b $\mathrm{F} 4 / 80^{+}$ macrophages (Fig 4.11C) within the tumor also showed a significant increase in TNF- $\alpha$ production due to WGP. Both an increased number of CD11b $\mathrm{b}^{+}$myeloid cells (Fig 4.11D, left) and an increase in the percent of CD11 ${ }^{+}$cells producing TNF- $\alpha$ (Fig 4.11D, right) significantly correlated with decreased tumor burden. Neither $\mathrm{CD} 4^{+}$nor $\mathrm{CD} 8^{+} \mathrm{T}$-cells showed increased IFN- $\gamma$, further supporting that the reduction in tumor burden was driven by the WGP-trained myeloid cells (Fig 4.11E). Similar antitumor effects were also observed in mice orthotopically implanted with Pan02 tumors (Fig 4.12A). To further confirm that innate immune cells are responsible for the observed anti-tumor immune responses, orthotopic KPC tumors were implanted into NSG mice. Similar to WT mice, NSG mice also showed a significant reduction in tumor size due to WGP training, confirming that the anti-tumor effects of WGP were driven by innate immune cells and functioned independently of adaptive responses (Fig 4.12B). Kalafati et al had shown that innate immune training of granulopoiesis promotes anti-tumor immunity [203]. While we had not seen an important contribution of granulocytes to our phenotype of trained immunity, we examined whether granulocytes are involved in WGP-dependent reduction in pancreatic tumors. We administered anti-Ly6G mAb or isotype control mAb on day -2 , 4, 10 and 16, injected mice with PBS or WGP on day 0 and implanted orthotopic KPC tumors on day 7. The depletion efficiency of neutrophils in the pancreas (Fig 4.12A) along 
with the pancreatic tumor burden were assessed (Fig 4.13B). Our results showed a significant reduction in tumor size in the WGP group in the absence of neutrophils.

Given that a complete inhibition of innate immune cell trafficking into the pancreas and training of pancreatic myeloid cells following WGP treatment in $\mathrm{CCR} 2^{-/-}$mice, we reasoned that $\mathrm{CCR} 2^{-/-}$mice would also not show the beneficial anti-tumor immune effects of WGP training. In line with this hypothesis, CCR2 $2^{--}$mice that received WGP did not show a reduced tumor burden (Fig 4.14) as compared to WT mice. We also utilized an admix model where sorted CD11b+ CCR2 ${ }^{-}$and $\mathrm{CCR} 2^{+}$cells were mixed with KPC tumor cells and implanted orthotopically into WT mice. Tumors implanted with CCR2+ cells showed a decreased tumor burden (Fig 4.15) Together, these findings demonstrate that CCR2 is requisite for the WGP-driven influx of trained innate immune cells into the pancreas and that those are consequential for the anti-tumor effects. CyTOF was also performed on these samples to see if there were phenotypic differences. A significant increase in the relative frequency of $\mathrm{CD} 8^{+} \mathrm{T}$-cells and a decrease in the relative percent of $\mathrm{CD}_{11 \mathrm{~b}^{+}}$myeloid cells were observed in the tumors that had been admixed with CCR2 ${ }^{+}$ cells (Fig 4.16).

Though CCL2-CCR2 signaling had been identified to be critical in the recruitment of trained monocyte-derived macrophages to the pancreas, we also wanted to know whether the presence of trained HSCs in the bone marrow alone was sufficient to recruit CCR2+ cells to the pancreas to slow the growth of orthotopic pancreatic tumors. A BM chimera model was used where B6/SJL (CD45.1) mice were treated with PBS or WGP and 7 days later their BM was harvested and transplanted into lethally irradiated (950 cGy) WT (CD45.2) mice. Six weeks following transplantation, recipient mice were implanted with 
orthotopic KPC tumors and tumor size was assessed 14 days later (Fig 4.17). Tumor size between the two groups was the same. First, this indicates that the mechanism of peripheral trained immunity in the pancreas is different than other models of trained immunity which appear to be entirely driven by the induction of trained immunity in the bone marrow. Second, this shows that the trafficking of WGP to the pancreas, which upregulates CCL2 signaling, is likely necessary to recruit trained monocytes from the bone marrow directly to the pancreas.

\section{Trained innate immunity synergizes with anti PD-L1 mAb therapy to prolong survival and can also be used in the treatment setting}

Although the significant reduction in tumor burden was shown due to WGP training, it is the case that all mice developed fatal tumors. Through scRNA-Seq analysis, we observed a distinct expression of CD274 (PD-L1) on myeloid clusters (Fig 4.18A). RNA-Seq data also showed that CD274 was upregulated following WGP training ( $\left.p=3.37 \times 10^{-4}\right)$. Analyses of orthotopic KPC tumors also indicated that while the tumor cells themselves did express some PD-L1, macrophages and DCs in the TME expressed the highest levels of PD-L1 (Fig 4.18B). This is important as it has been reported that host expression of PDL1 determines the efficacy of PD-L1 pathway blockade-mediated tumor regression. This leads us to hypothesize that WGP training may act synergistically with anti-PD-L1 mAb to further prolong mouse survival [204].

To assess whether anti-PD-L1 mAb therapy synergizes with WGP-induced trained immunity in the pancreas, mice were given WGP on day -7 , implanted with orthotopic $\mathrm{KCP}$ tumors on day 0 and were then given 3 treatments of either anti PD-L1 mAb or rat 
IgG2b isotype control $\mathrm{mAb}$. As has been shown in several clinical trials, anti-PD-L1 therapy alone failed to prolong survival even beyond that of the $\operatorname{IgG} 2 \mathrm{~b}$ isotype control $\mathrm{mAb}$ treated mice (Fig 4.18C). WGP-trained mice did survive significantly longer than IgG2b isotype and anti-PD-L1treated mice. However, combination of WGP and anti-PD-L1 together prolonged survival most effectively. This shows that there is a clinical benefit to combining WGP with anti PD-L1 immuno-checkpoint blockade therapy.

Thus far, the use of WGP has been described in a setting in which WGP is administered before tumor cells are implanted. Considering the reduction in tumor size of this model, we also tested a more clinically relevant model in which mice were implanted with orthotopic KPC tumors and WGP was used to incite trained immunity thereafter. Two doses were given; the first after mice had recovered from surgery and a second one week later (Fig 4.19). In the therapeutic setting, the WGP-driven influx of trained myeloid cells to the pancreas was also shown to prolong survival. Together, these data suggest that the initiation of trained immunity in the pancreas using WGP has relevant clinical applications in treating pancreatic cancer that necessitate further translational research and investigation. Finally, we wanted to investigate whether WGP combined with anti-PD-L1 may prolong survival in the therapeutic setting so mice were treated with WGP on day 4 and 11 following tumor implantation, and were then given three doses of anti-PD-L1. Similar to previous results, mice treated with monotherapy of anti PD-L1 did not have a survival benefit beyond untreated mice, WGP treated mice did show prolonged survival as compared to PBS and anti-PD-L1 treated mice, and mice treated with WGP and PD-L1 showed the most prolonged survival (Fig 4.20). 


\section{CHAPTER IV FIGURES}

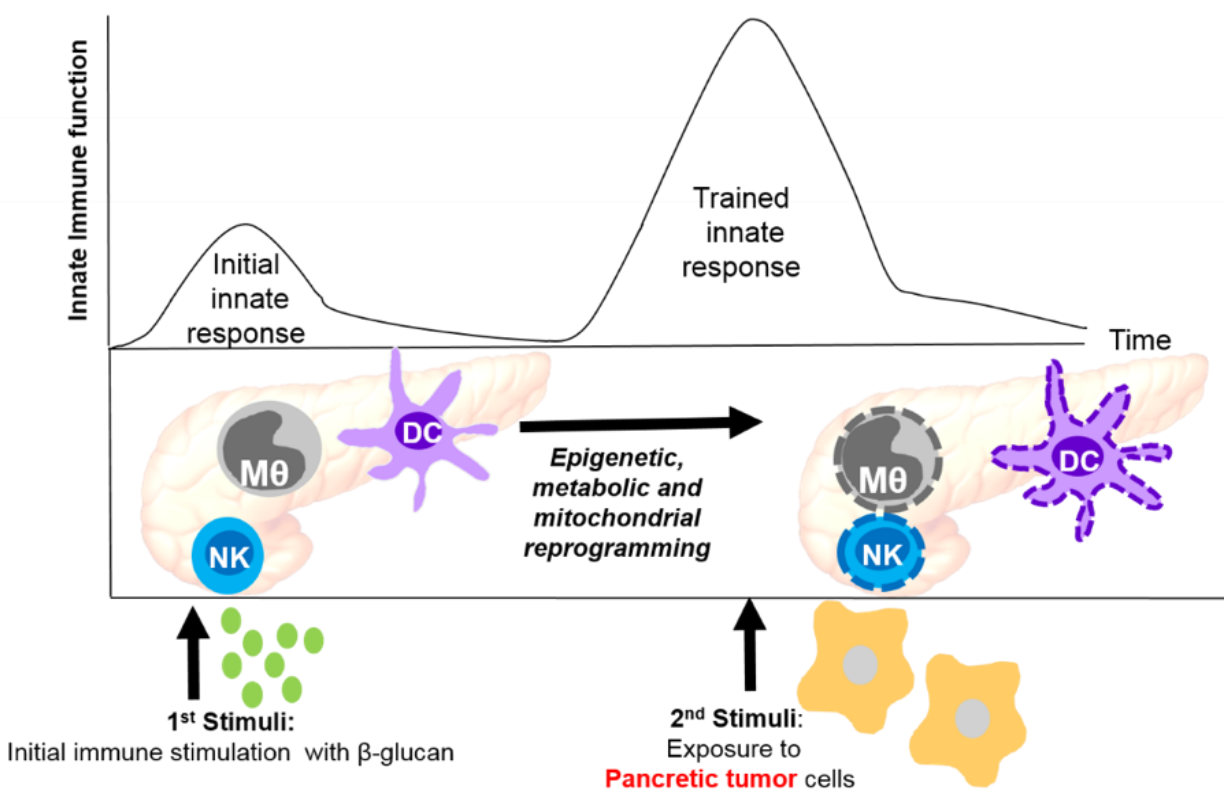

Figure 4.1: Schematic of the anti-tumor effects of WGP-induced trained immunity in

\section{the setting of pancreatic cancer}

Injection of WGP and trafficking of WGP to the pancreas causes the influx of trained innate immune cells to the pancreas. These cells have gone epigenetic, metabolic, mitochondrial and transcriptional reprograming and when they then come into contact with pancreatic tumor cells, the trained cells are re-stimulated. 

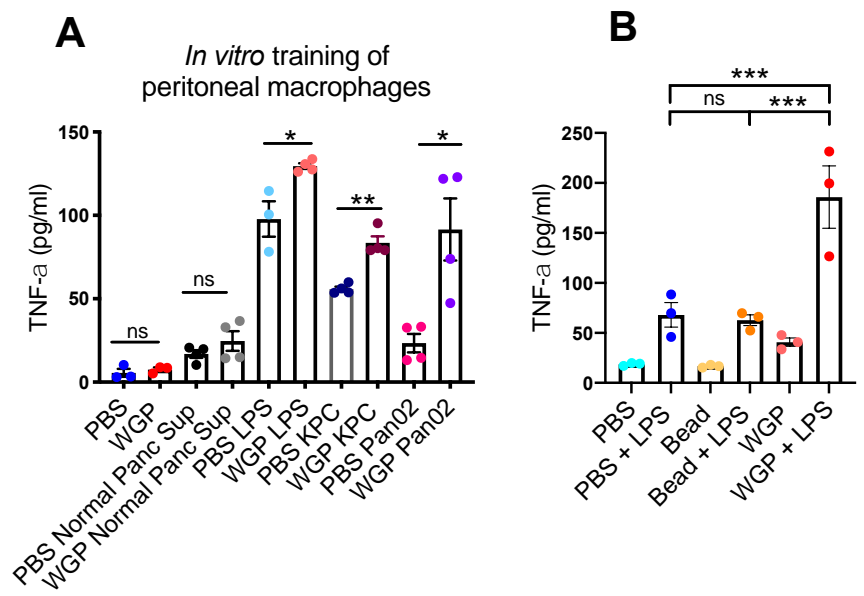

\section{Figure 4.2: Tumor conditioned media elicits a trained response in vitro}

(A) Peritoneal macrophages were acquired from WT mice and were stimulated in vitro with PBS or WGP for 24 hours. Cells were allowed a resting period of 7 days followed by restimulation with PBS, LPS, the supernatant from cultured and untreated pancreatic cells, or the supernatant from cultured KPC or Pan02 pancreatic cancer cells for 24 hours. The amount of TNF $\alpha$ released over the 24 hours was quantified using an ELISA. (B) Peritoneal macrophages were acquired from WT mice and were stimulated in vitro with PBS, WGP, or $3 \mu \mathrm{m}$ polystyrene beads for 24 hours. Cells were allowed a resting period of 7 days followed by a 24 hour restimulation with PBS or LPS. The amount of TNF $\alpha$ released over the 24 hours was quantified using an ELISA. Data are represented as mean \pm SEM. ns= non-significant; $* p<.05, * * p<.01, * * * p<.001, * * * * p<.0001$ 
A
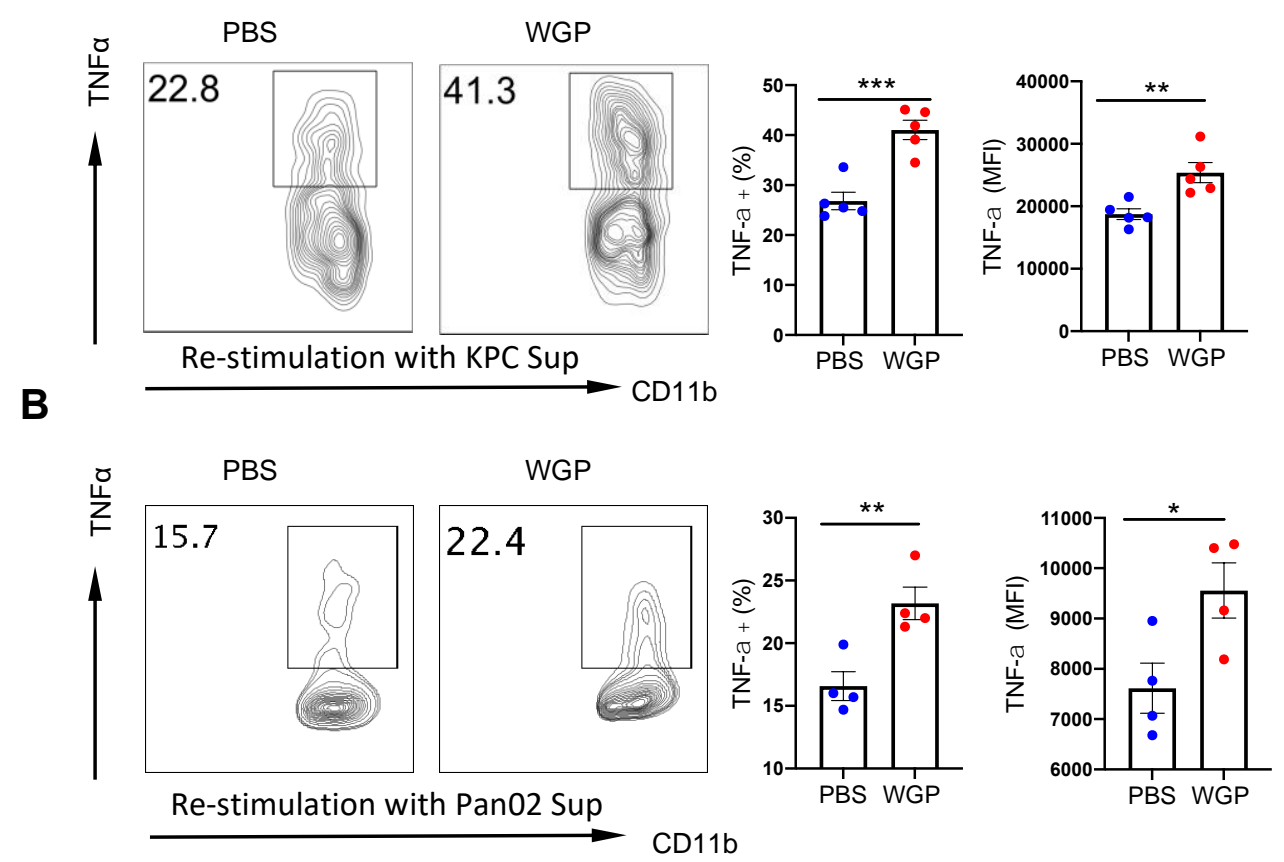

Figure 4.3: Tumor conditioned media elicits a trained response ex vivo

$(\mathbf{A}+\mathbf{B})$ WT mice were treated with PBS or WGP and 7 days later pancreatic cells were processed and restimulated with the supernatant from cultured (A) KPC or (B) Pan02 pancreatic cancer cells. Data are represented as mean \pm SEM. ns $=$ non-significant; $* p<.05$, $* * p<.01, * * * p<.001$ 
A

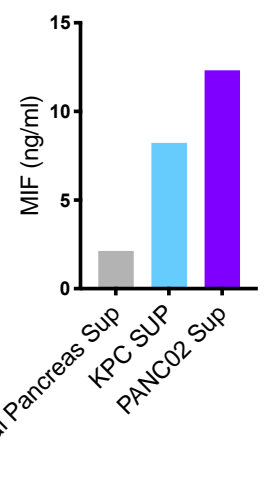

B

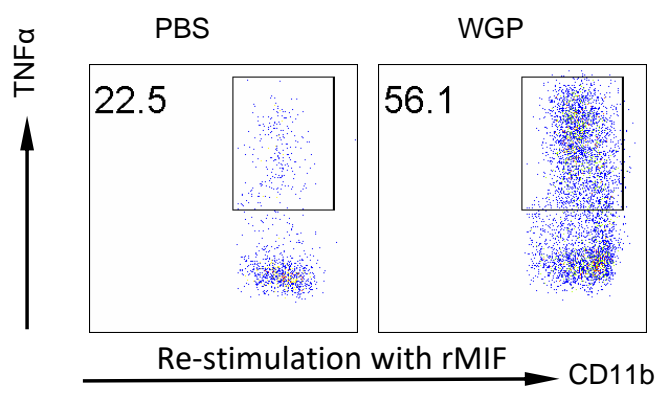

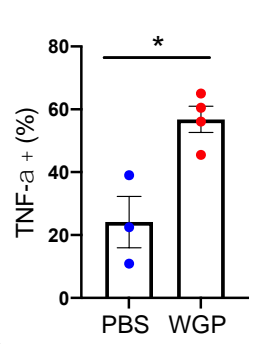

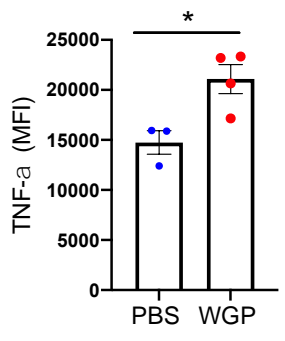

Figure 4.4: MIF secreted by tumor cells elicits the trained response

(A) The amount of rMIF in the supernatant from cultured untreated WT pancreatic cells, KPC cells and Pan02 cells was measured using an ELISA. (B) WT mice were treated with PBS or WGP and 7 days later pancreatic cells were processed and restimulated with 10 $\mathrm{ng} / \mathrm{mL}$ of rMIF. Data are represented as mean \pm SEM. $\mathrm{ns}=$ non-significant; ${ }^{*} p<.05$
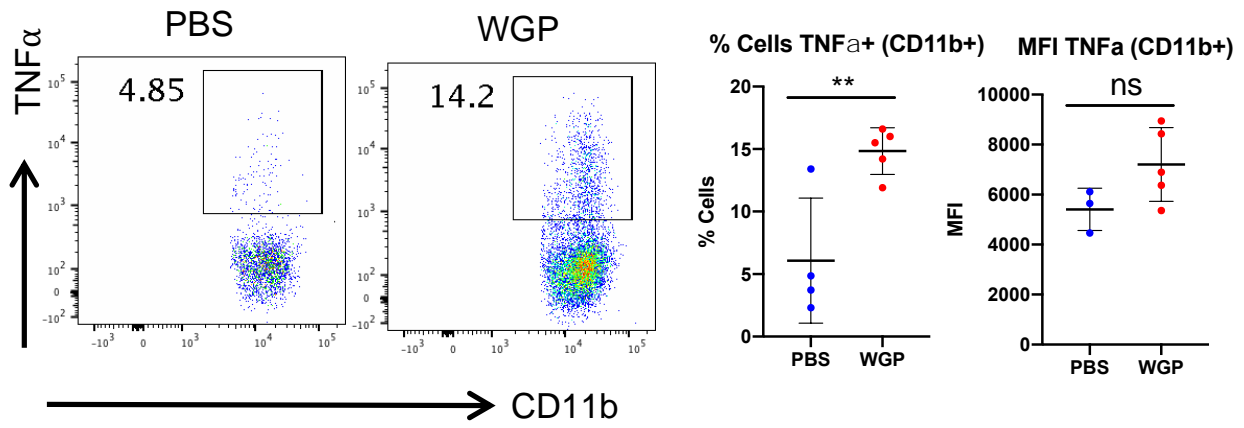

Figure 4.5: Recombinant HMGB1 elicits the trained response

WT mice were treated with PBS or WGP and 7 days later pancreatic cells were processed and restimulated with $10 \mathrm{ng} / \mathrm{mL}$ of mouse HMGB1. Data are represented as mean \pm SEM. $\mathrm{ns}=$ non-significant; $* * p<.01$ 

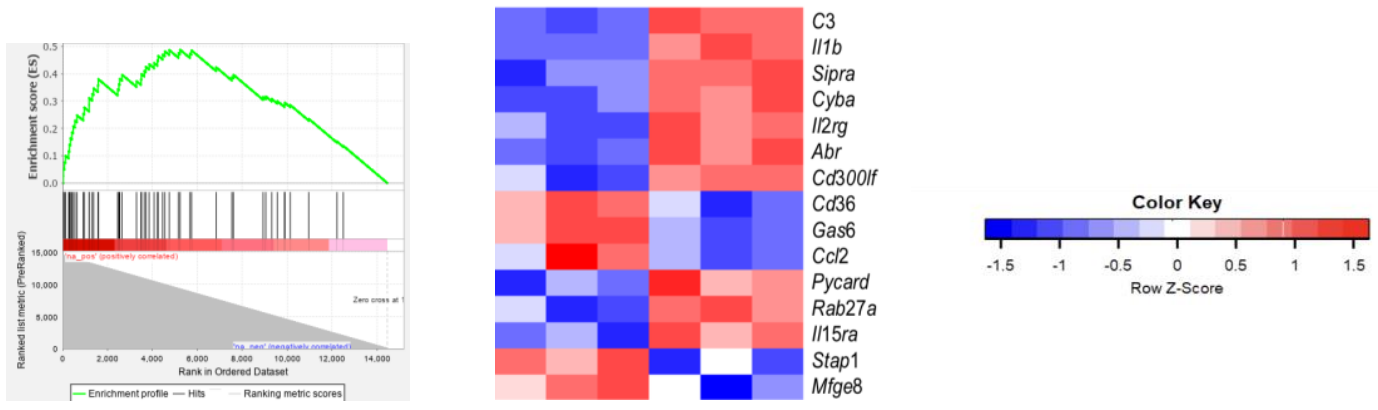

Figure 4.6: WGP treatment positively regulates genes realtedto phagocytic cellular processes

Enrichment plots (GSEA) and heat map of genes related to the positive regulation of phagocytosis in $\mathrm{CD}_{1} 1 \mathrm{~b}^{+}$cells from 7-day WGP-trained as compared to PBS mice 

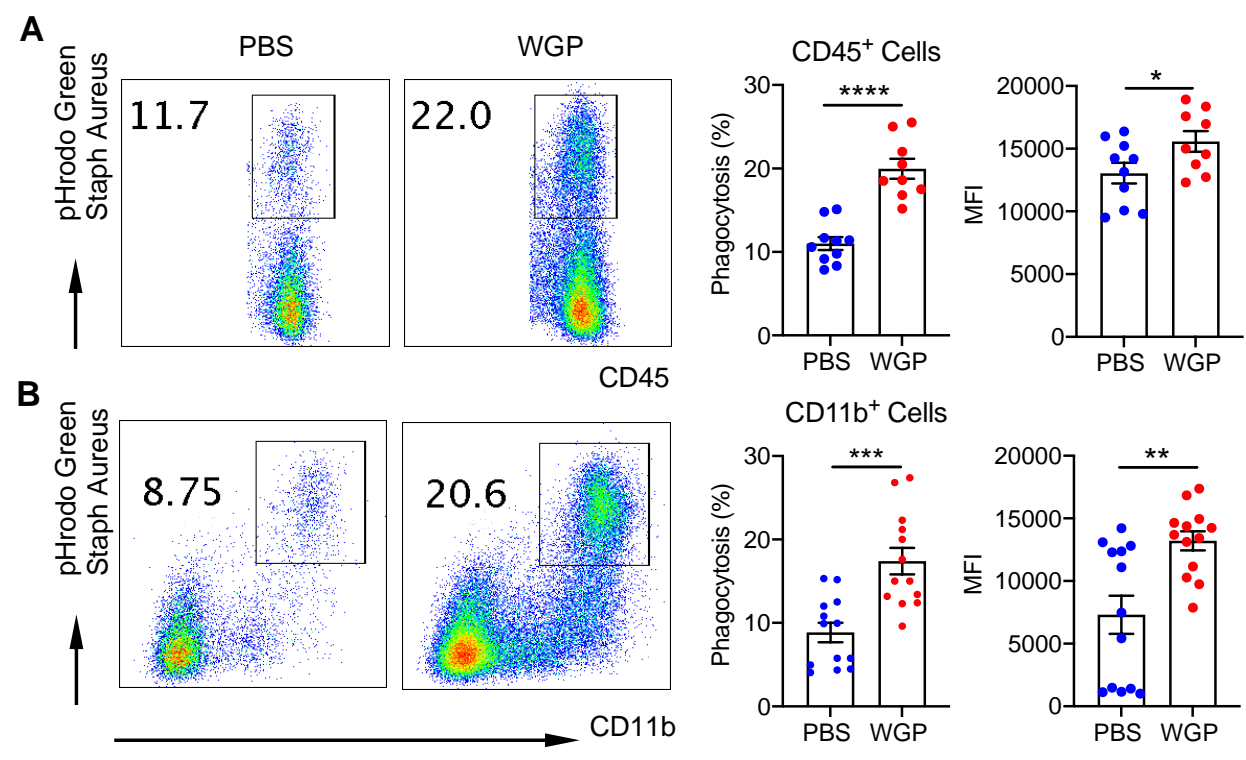

Figure 4.7: WGP training increases phagocytosis of a pHrodo Green Staph Aureus particle

(A) The percent of $\mathrm{CD} 45^{+}$pancreatic cells that phagocytosed a pHrodo Green Staph Aureus particle in PBS and 7-day WGP mice along with the MFI of the pHrodo Green Staph Aureus particle. (B) The percent of CD11b+ myeloid pancreatic cells that phagocytosed a pHrodo Green Staph Aureus particle in PBS and 7-day WGP mice. Cells were first gated on the $\mathrm{CD} 45^{+}$population. The MFI of the pHrodo Green Staph Aureus particle is shown. Data are represented as mean \pm SEM. $* p<.05, * * p<.01, * * * p<.001, * * * * p<.0001$ 

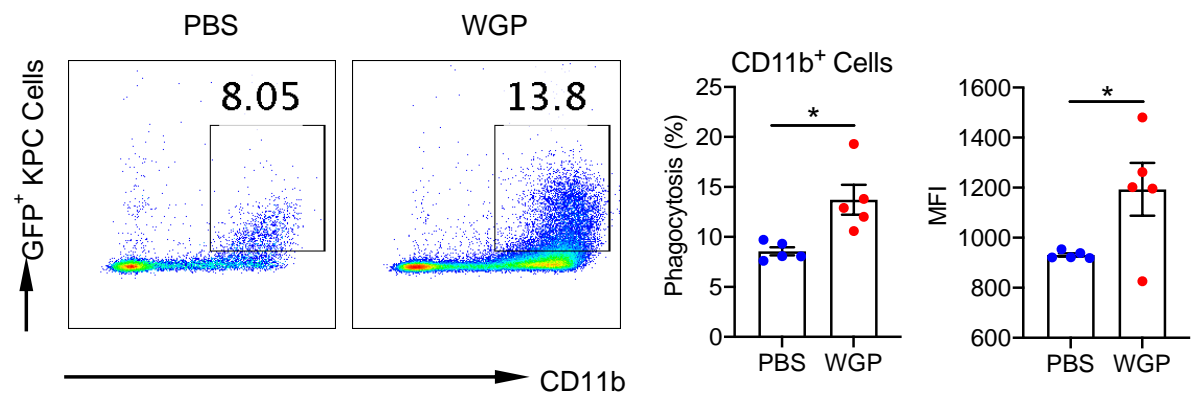

Figure 4.8: WGP training increases phagocytosis of KPC tumor cells

The percent of CD11 $\mathrm{b}^{+}$myeloid pancreatic cells that phagocytosed $\mathrm{KPC}^{\mathrm{GFP}+}$ tumor cells in PBS and 7-day WGP mice. Cells were first gated on the $\mathrm{CD}^{4} 5^{+}$population. The MFI of the $\mathrm{GFP}^{+}$signal is shown. Data are represented as mean $\pm \mathrm{SEM} . * *^{*}<. .01$ 
A GO_ROS Biosynthetic Process

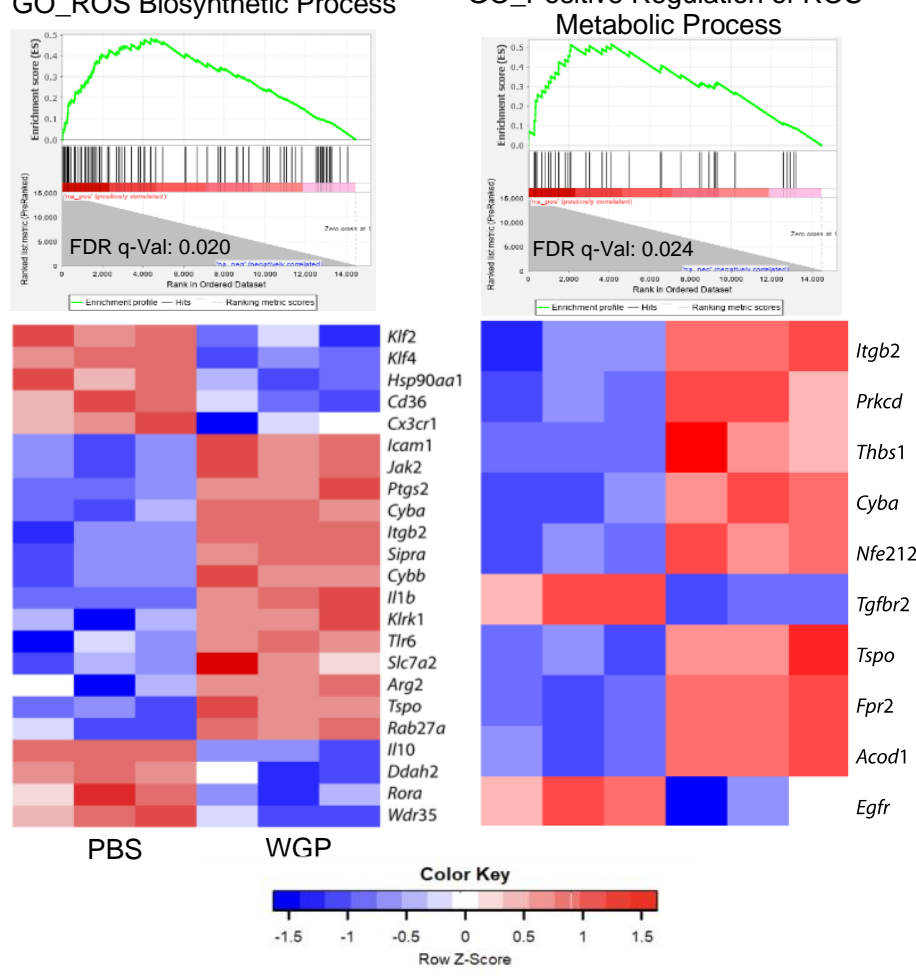

B

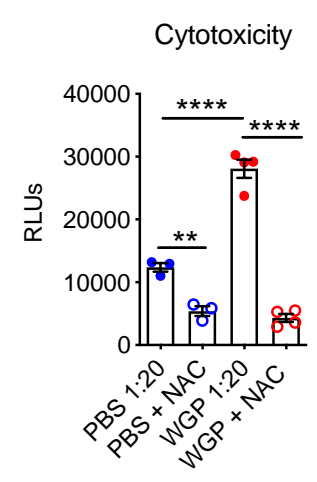

Figure 4.9: WGP treatment enhances cytotoxicity of myeloid cells against tumor cells

(A) Enrichment plots (GSEA) and heat map of genes related to the reactive oxygen species biosynthetic processes and the positive regulation of reactive oxygen species metabolic

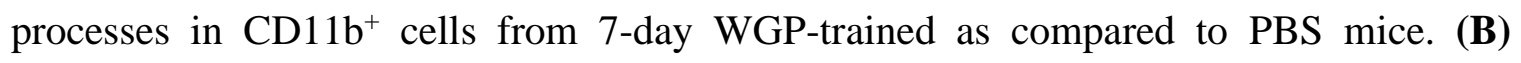
Summarized results from a cytotoxicity assay where CD11b cells from PBS and 7-day

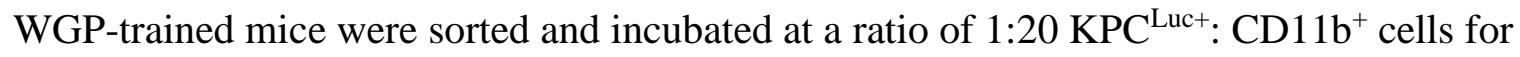
24 hours. NAC was used to block ROS expression and the tumor cytotoxicity was assessed by measuring luminescence (PBS $n=3$, WGP $n=4$ ). Data are represented as mean \pm SEM. 


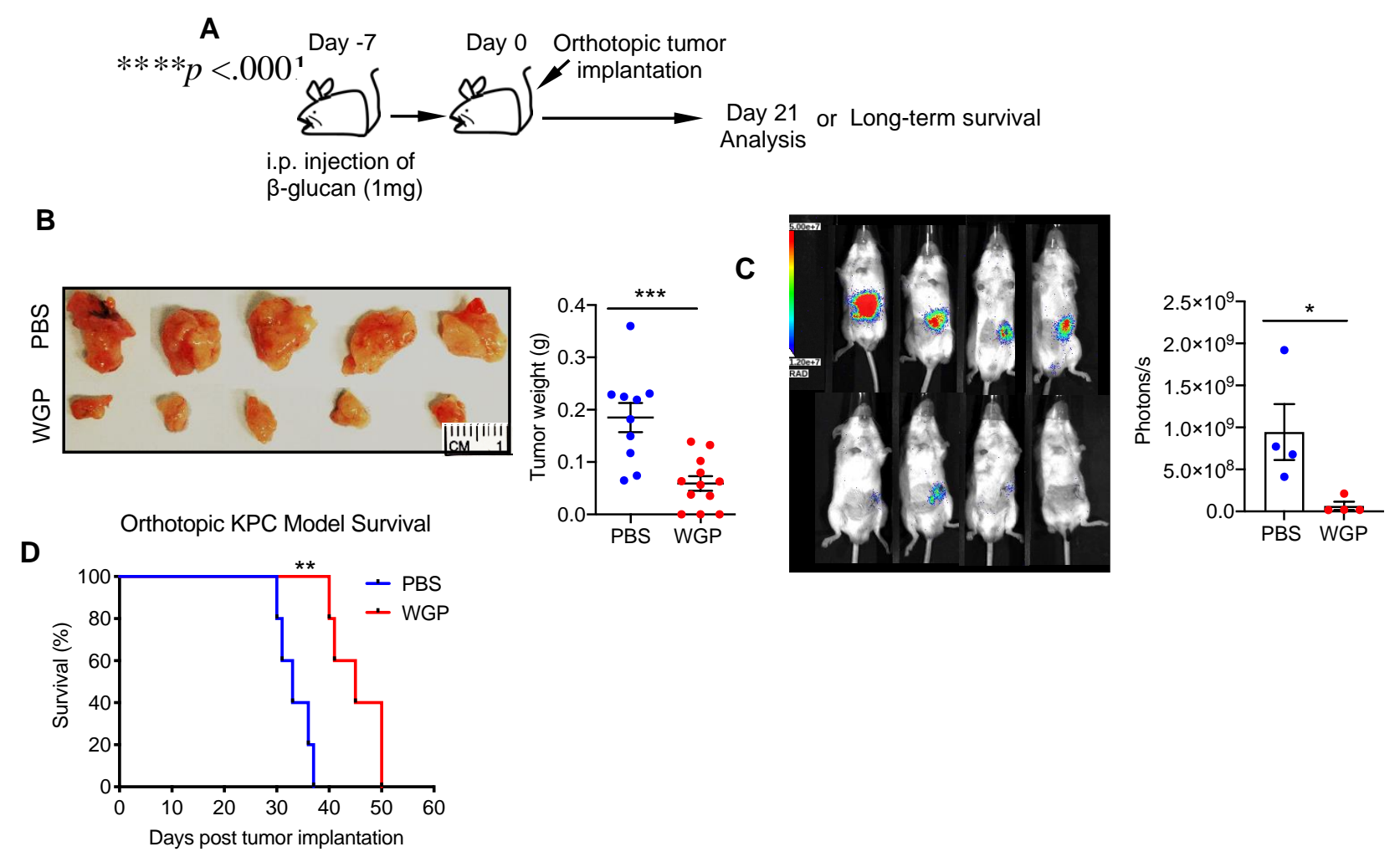

Figure 4.10: WGP treatment decreases tumor size in an orthotopic model of pancreatic cancer

(A) Experimental schema. (B) C57BL/6 mice received a single IP injection of WGP or PBS and 7 days mice were implanted orthotopically with KPC pancreatic cancer cells. Representative pictures of tumors and quantitative analysis of tumor weight are shown. Tumor weight was measured at day 21 (PBS n=5, WGP n=5). (C) C57BL/6 mice received a single i.p. injection of WGP or PBS and 7 days later were implanted orthotopically with $\mathrm{KPC}^{+\mathrm{Luc}}$ pancreatic cancer cells. On day 21post tumor implantation, mice were given I.P. luciferin bioluminescent substrate and were placed in a photon imager to measure tumor size in vivo. Data are represented as mean \pm SEM. ${ }^{*} p<.05, * * p<.01, * * * p<.001$ 


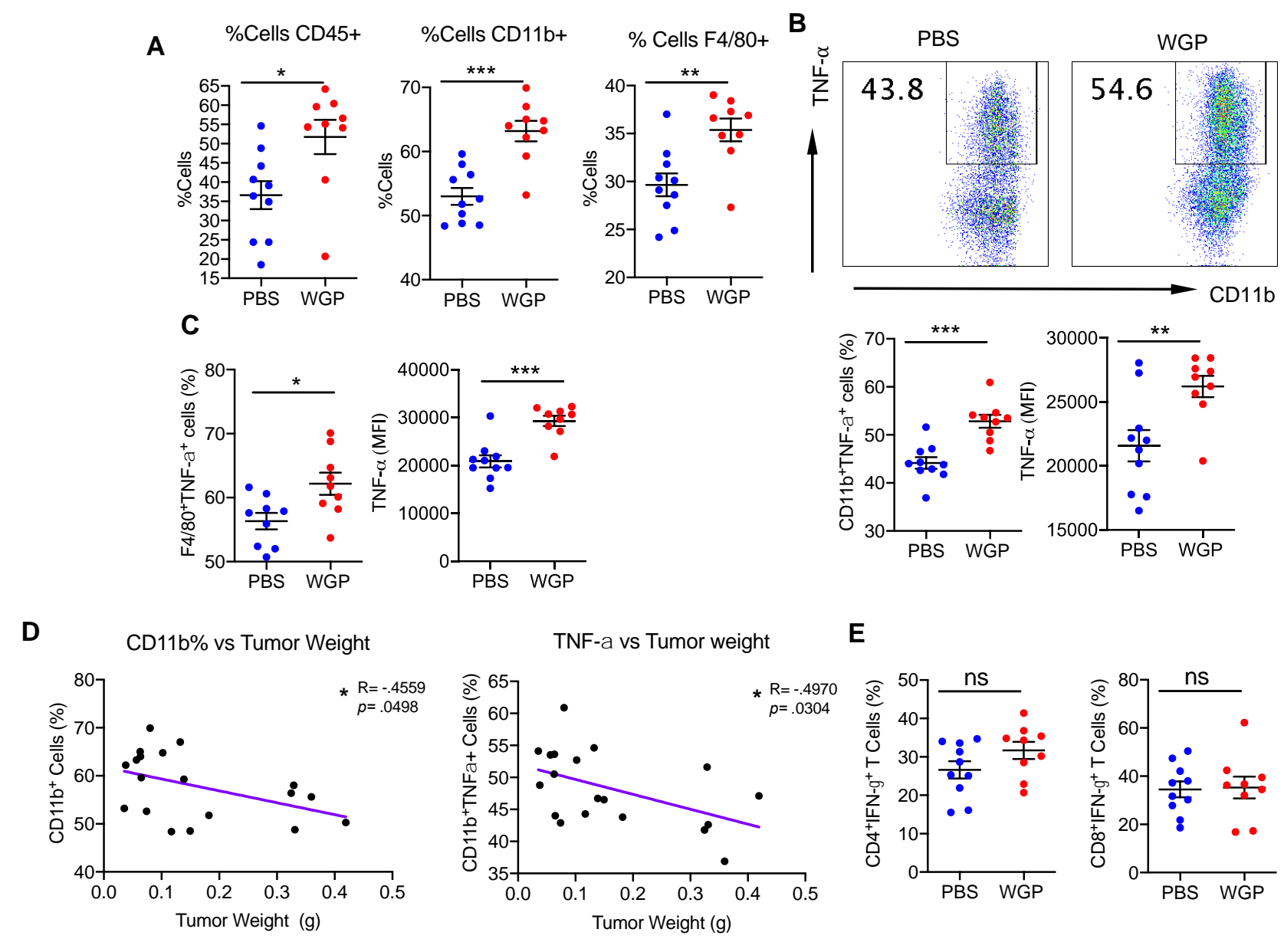

Figure 4.11: Immune phenotyping of PBS vs WGP treated pancreatic KPC tumors

(A) Phenotyping of the tumors showing the percent of viable cells that are $\mathrm{CD} 45^{+}$, the percent of the $\mathrm{CD}_{4} 5^{+}$population that are $\mathrm{CD} 11 \mathrm{~b}^{+}$, and the percent of $\mathrm{CD} 11 \mathrm{~b}^{+}$cells that are F4/80 ${ }^{+}$. (B) TNF $\alpha$ production in CD11 $\mathrm{b}^{+}$cells from PBS and 7-day WGP-trained that were restimulated with LPS. Percent of $\mathrm{TNF}^{+}$cells and the MFI of $\mathrm{TNF} \alpha$ are shown. (C) Summarized data of TNF $\alpha$ production in $\mathrm{CD} 11 \mathrm{~b}^{+} \mathrm{F} 4 / 80^{+}$cells from PBS and 7-day WGPtrained that were restimulated with LPS. Percent of TNF $\alpha^{+}$cells and the MFI of TNF $\alpha$ are shown. (D) Tumor weight was correlated with the percent of $\mathrm{CD} 45^{+}$immune cells that were 
$\mathrm{CD} 11 \mathrm{~b}^{+}$(left) and the percent of $\mathrm{CD} 45^{+} \mathrm{CD} 11 \mathrm{~b}^{+} \mathrm{TNF} \alpha^{+}$cells (right). (E) Summarized data of the percent of CD4+ and CD8+ T-Cells expressing IFN $\gamma$. Data are represented as mean \pm SEM. Pearson correlation coefficients were used to measure the strength of the linear associations. ${ }^{*} p<.05, * * p<.01, * * * p<.001$.

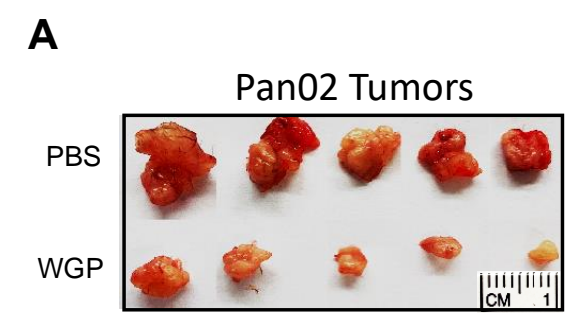

B

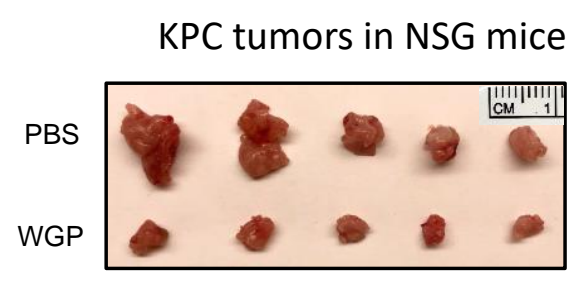

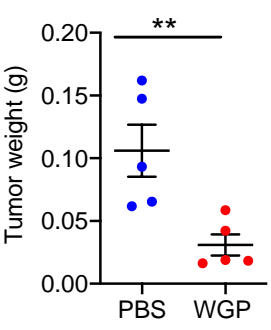

NSG KPC Tumor

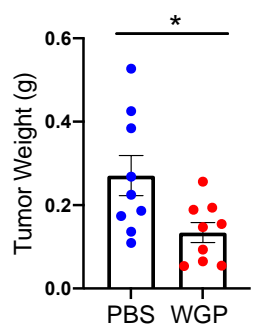

\section{Figure 4.12: Additional tumor models to test the anti-tumor effects of WGP}

(A) C57BL/6 mice received a single i.p. injection of WGP or PBS and 7 days mice were implanted orthotopically with Pan02 pancreatic cancer cells. Representative pictures of tumors and quantitative analysis of tumor weight are shown. Tumor weight was recorded at day 21 (PBS n=5, WGP n=5). (B) NSG mice were treated with either PBS or WGP and 7 days later orthotopic KPC pancreatic tumors were implanted. 21 days following tumor implantation, mice were euthanized and tumor weight was recorded. Data are represented as mean \pm SEM. ${ }^{*} p<.05, \mathrm{P}^{* *<}<.01$. 
A

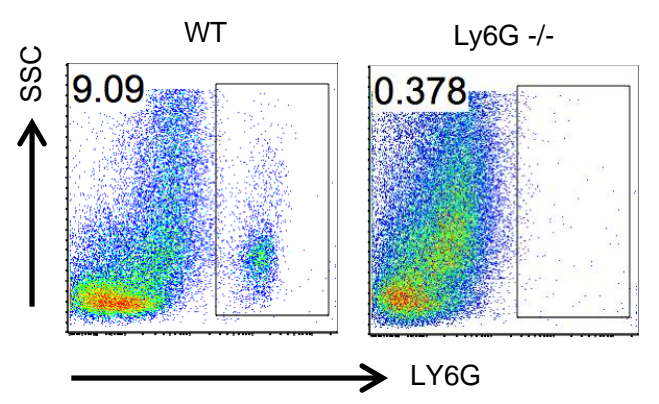

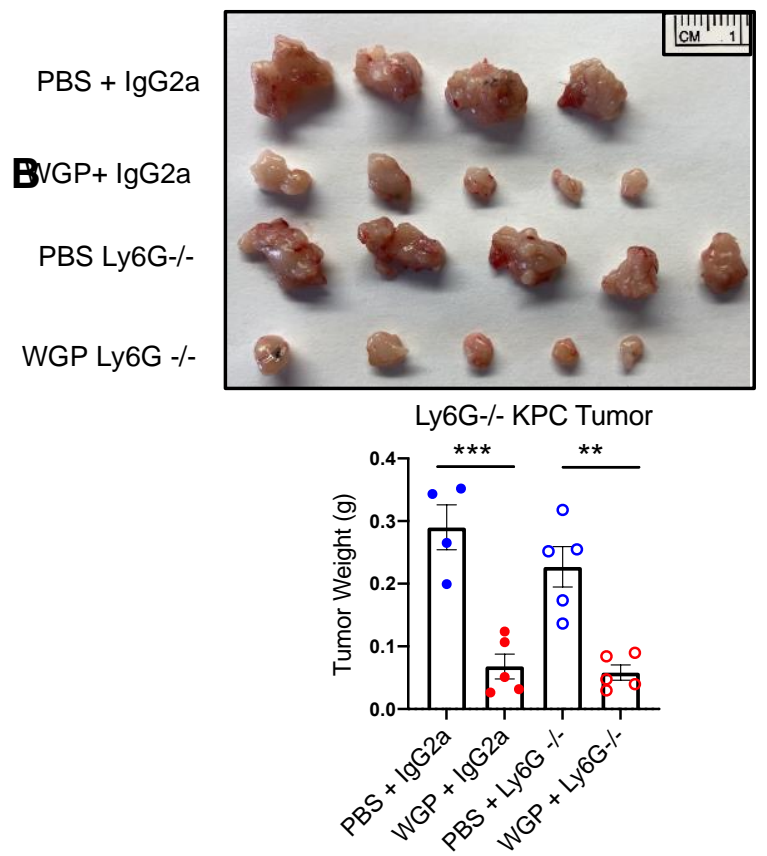

Figure 4.13: Neutrophils are not involved in the anti-tumor effects of WGP

$(\mathbf{A}+\mathbf{B})$ To deplete neutrophils, mice were treated with anti-Ly6G mAb or anti-rat IgG2a isoype control on day -2. On day 0 mice were treated with PBS or WGP and on day 7 mice were implanted with orthotopic KPC pancreatic tumors. Additional doses of the anti-Ly6G $\mathrm{mAb}$ or anti-rat $\operatorname{IgG} 2 \mathrm{a}$ isoype control were given on days 4, 10 and 16 to maintain the Ly6G depletion. On day 21, mice were euthanized and (A) the depletion efficiency was assessed and (B) tumor weight was measured. Data are represented as mean \pm SEM. $* * p$ $<.01, * * * p<.001$. 

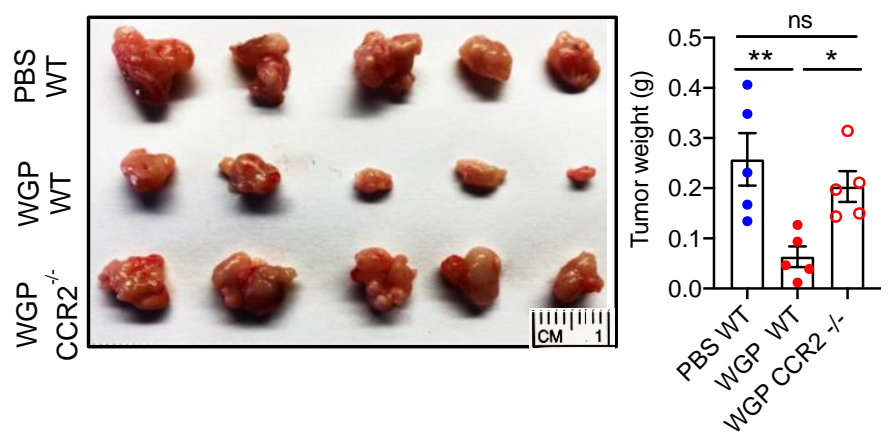

\section{Figure 4.14: WGP does not reduce tumor burden in CCR2 ${ }^{-/-}$mice}

C57BL/6 were treated with PBS or WGP and CCR2 $2^{-/-}$mice were treated with WGP and 7 days later were implanted with orthotopic KPC pancreatic tumor cells. Tumor weight and size was monitored for 3 weeks after implantation and tumor weight at day 21 is reported. (WT PBS n=5, WT WGP n=5, CCR2-/- WGP n=5). Data are represented as mean \pm SEM. $\mathrm{ns}=$ non-significant; $* p<.05, * * p<.01$.
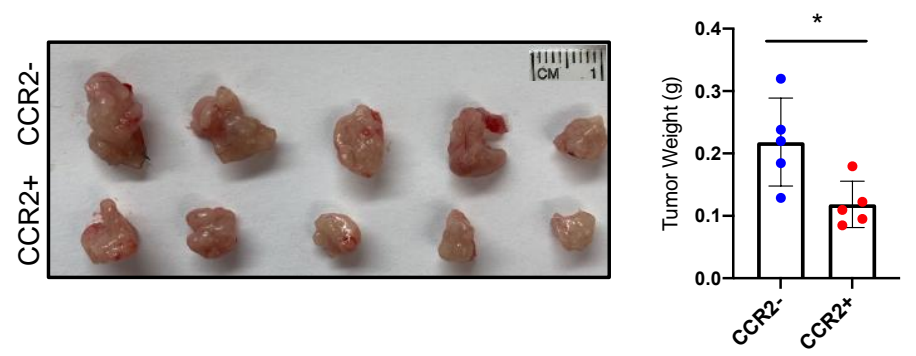

Figure 4.15: CCR2+ and CCR2- admix tumor model

WT mice were treated with WGP and 7 days later the CD11b+ and CD11b- populations were sorted, mixed with KPC tumor cells and implanted orthotopically into WT mice. 21 days after tumor implantation, mice were euthanized and tumor size was measured. 
$(\mathrm{CCR} 2+\mathrm{n}=5, \mathrm{CCR} 2-\mathrm{n}=5)$. Data are represented as mean \pm SEM. $\mathrm{ns}=$ non-significant; $* p$ $<.05$
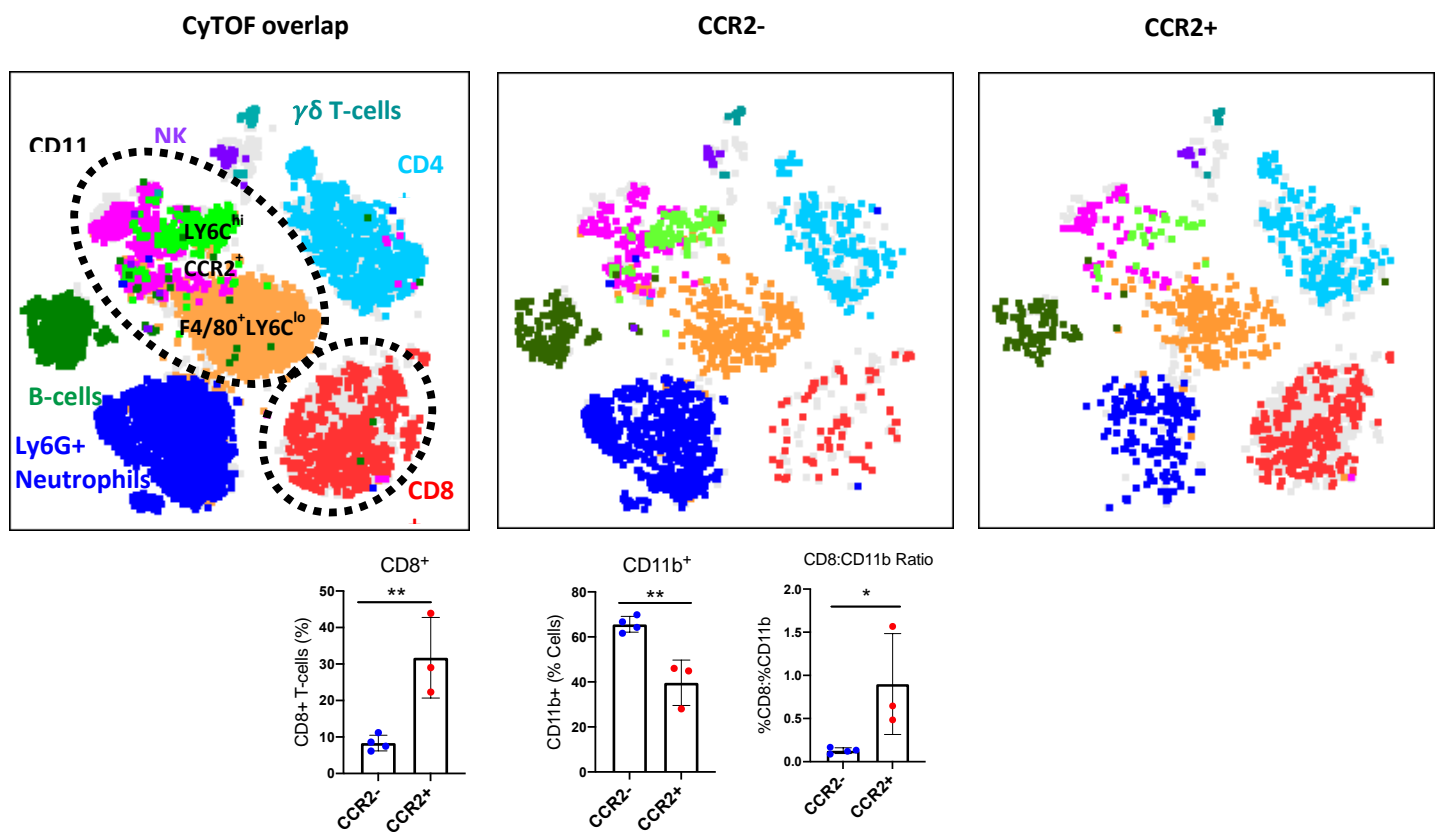

Figure 4.16: CyTOF of admix CCR2+ and CCR2- admix KPC tumors

CyTOF analysis was performed on CCR2+ and CCR2- admixed tumors. The percent of $\mathrm{CD} 8^{+}$and $\mathrm{CD} 11 \mathrm{~b}^{+}$cells in the tumors was assessed and is shown in representative images.

Data are represented as mean \pm SEM. $* p<.05, * * p<.01$
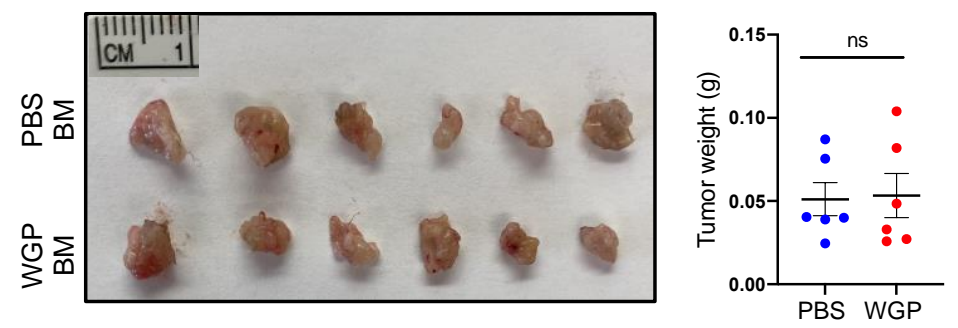

Figure 4.17: Bone marrow chimera model to assess the role of central trained immunity in orthotopic pancreatic tumors 
B6/SJL (CD45.1) mice were treated with PBS or WGP and 7 days later $2 \times 10^{6} \mathrm{BM}$ cells were transplanted into lethally irradiated WT (CD45.2) mice. 6 weeks after transplantation, recipient mice were implanted with orthotopic KPC tumors and tumor size was evaluated 14 days later (PBS $n=6$, WGP $n=6$ ) Data are represented as mean \pm SEM. $n s=$ nonsignificant.

A

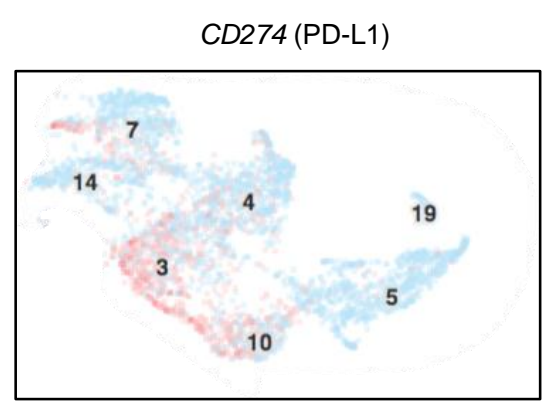

C

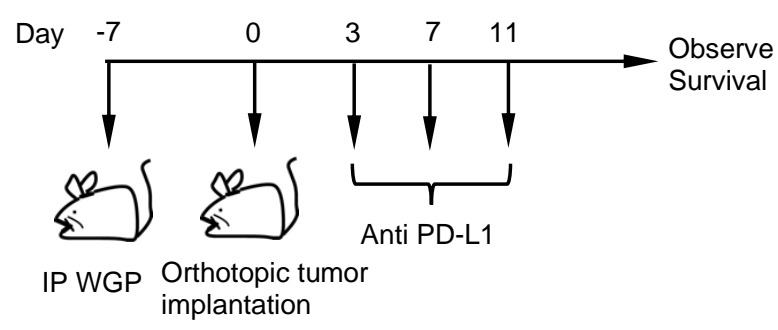

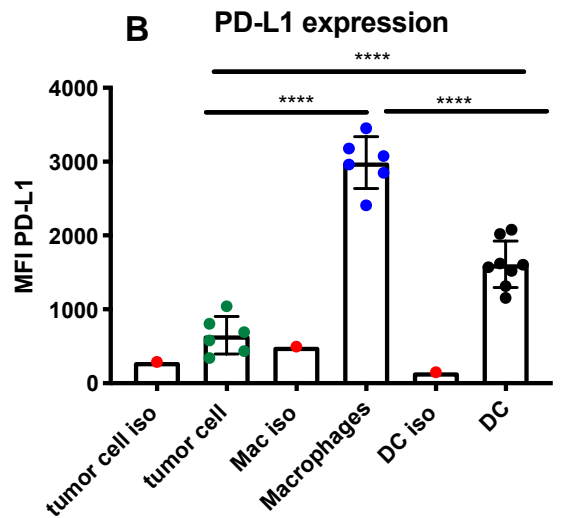

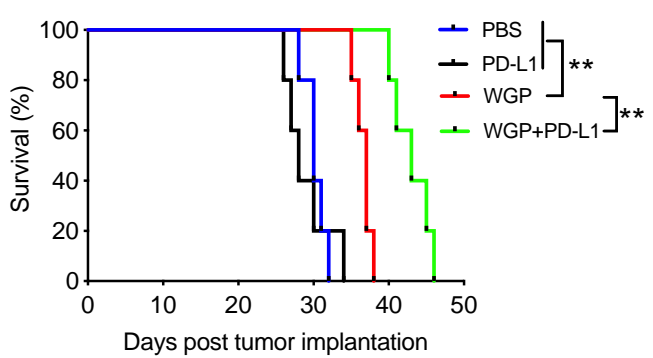

Figure 4.18: WGP synergizes with anti-PD-L1 immunotherapy to prolong survival

(A) scRNA-Seq data showing a UMAP of the myeloid clusters expressing CD274. (B) Experimental schema of WGP and anti PD-L1 therapy. Mice (n=5) were treated with PBS or WGP and 7 days later were implanted with orthotopic KPC pancreatic tumors. On days 3,7 and 11 post-tumor implantation, mice were given anti-PD-L1 mAb or anti-rat IgG2b $\mathrm{mAb}$ isotype control. Survival of mice was monitored. $* * p<.01, * * * * \mathrm{P}<.0001$ 

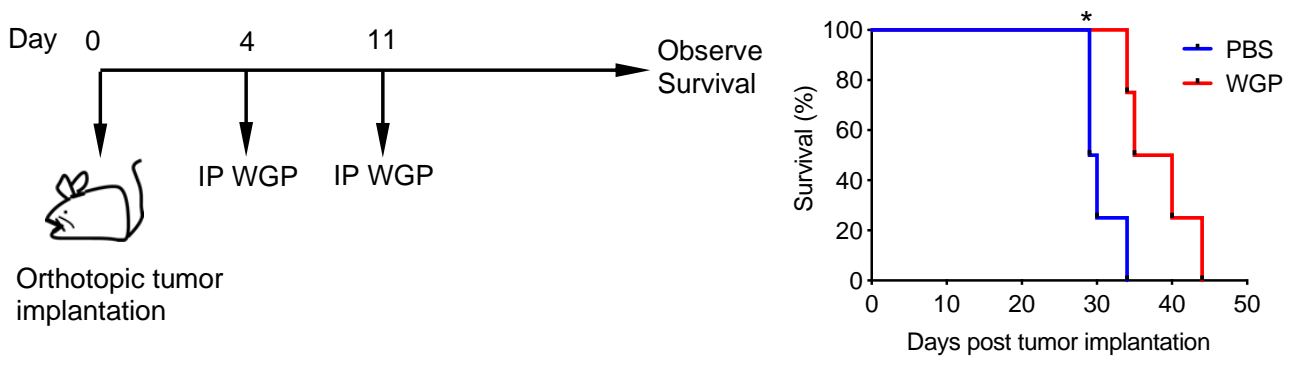

\section{Figure 4.19: WGP used in the adjuvant setting}

Experimental schema of WGP used in the therapeutic setting. Mice were implanted with orthotopic KPC pancreatic tumors and were given WGP once mice had recovered from the surgery at day 4 , and one week later on day $11 .{ }^{*} p<.05$.

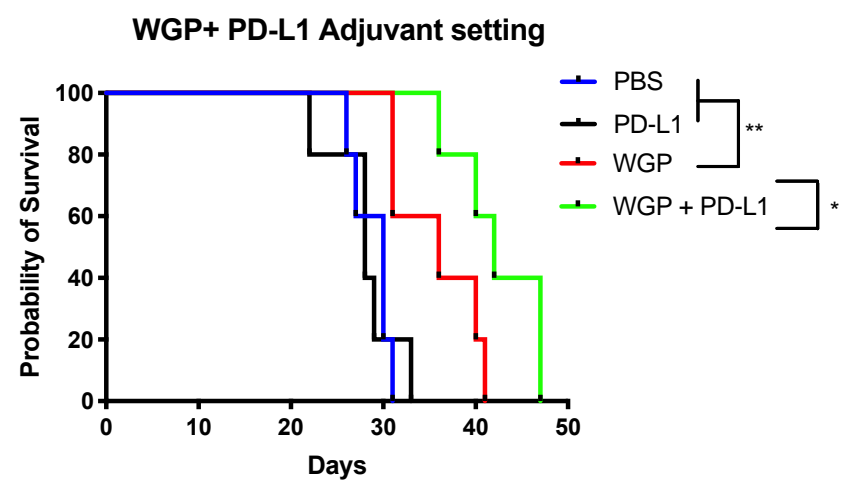

Figure 4.20: WGP can be combined with anti-PD-L1 mAb therapy in the adjuvant setting

Mice were treated with PBS or WGP on day 4 and 11, and were then given $100 \mu \mathrm{g}$ of antiPD-L1 mAb on days 13, 17 and 21. Survival was assessed. 


\section{CHAPTER IV DISCUSSION}

As we show that peripheral trained immunity has been established in the pancreas, an important question is whether this nonspecific innate immunological memory could be reactivated by tumor cells or their secreted factors. Re-exposure of in vivo WGP-trained pancreatic myeloid cells to tumor conditioned media vigorously elicits a potent trained response. While Kalafati et al recently showed a role for granulocytes in trained immunity driven anti-tumor mechanisms [203], the currently reported study is the first instance suggesting that myeloid cells in a specific organ can be trained to react directly to tumor cells of that same organ. Further, while we highlight that tumor-conditioned media can reactivate trained myeloid cells, we also identify specific factors in the tumor-conditioned media that are involved in this activation. MIF, which is known to be primarily secreted by tumor cells and whose primary targets are myeloid cells [205, 206], was found to be actively secreted by both Pan02 and KPC tumor cells. Using this average concentration of rMIF to re-stimulate WGP-trained cells showed that MIF can activate trained myeloid cells. We also show that HMGB1 can elicit the trained response. Others have reported that DAMPs can be involved in trained immunity, however have primarily shown that they can act as the initial training stimulus [207]. It has not been previously shown that DAMPs secreted by tumor cells can re-stimulate trained cells. This understanding has implications for which cancer cell types may be most capable of re-stimulating trained cells based on the DAMPs that that cell type secretes. Furthermore, there are likely other tumor-secreted 
factors that may also function to re-activate trained myeloid cells and may activate them to different extents, and further investigation into these specific factors may shed more light on the anti-tumor capabilities and mechanisms of trained immunity. Ultimately, we also identify the novel concept that specific factors released by tumors and tumor cells themselves can act as the second signal in the incitement of trained immunity.

While often the anti-tumor mechanisms of myeloid cells are thought to be indirect and orchestrated through the activation of adaptive immune cells, here we show that WGP training upregulates the direct anti-tumor functionalities of myeloid cells in the pancreas. Due to WGP training we observed enhanced phagocytosis of tumor cells and ROS mediated cytotoxicity to tumor cells. Inhibiting ROS production abrogates the cytotoxicity of WGP-trained myeloid cells. Supporting results of the cytotoxicity assay, CyTOF data, RNA-Seq data and scRNA-Seq data all identify substantial enhancements in the expression of ROS-related molecules/genes in these trained cells, though other groups have submitted that training by $\beta$-glucan is not associated with increased production of ROS by macrophages/monocytes $[125,208]$. This finding suggests that the phenotype of trained immunity induced by particulate $\beta$-glucan in the pancreas may be unique. Supporting our results, several studies have characterized the mechanism of BCG-trained macrophages cytotoxicity against bladder cancer cells. Such studies indicate that the cytotoxic effects are due to both enhanced cytokine production and the production of ROS [209-211]. It is also worth noting that while our findings show that ROS production is an important contributor to the anti-tumor phenotype, in the complex tumor setting there are likely additional pathways that play a significant role in the tumor surveillance and control performed by trained myeloid cells. 
We then translate our findings regarding the anti-tumor potential of the trained innate myeloid cells that enter the pancreas to orthotopic models of pancreatic cancer, where we observe a dramatic decrease in the tumor size and an increase in the survival of mice given only one I.P administration of WGP. We also observe a reduction of tumor size in mice with both KPC and Pan02 tumors, revealing that these myeloid-driven anti-neoplastic effects are broadly applicable to pancreatic tumors. Importantly, through the depletion of neutrophils, along with the use of NSG mice and $\mathrm{CCR} 2^{-/-}$mice, we demonstrate that the cells responsible for tumor control are innate myeloid-derived cells that traffic to the pancreas through CCL2-CCR2 signaling.

BM chimeric models support that the presence of trained immunity within HSCs in the bone marrow is not enough on its own to slow the growth of pancreatic tumors, which is in contrast to other proposed models of peripheral trained immunity. Instead, the early trafficking of WGP to the pancreas and the resulting production of CCL2 by cells in the pancreas recruit trained $\mathrm{CCR} 2^{+}$myeloid cells from the circulation and periphery, which contribute to the antitumor effects. It is important to point out that given how quickly trained immune cells arrive in the pancreas, it is unlikely that these cells are directly from the bone marrow, though further investigations are needed to ascertain the source of incoming trained myeloid cells. We also show that several new populations of macrophages and monocytes exist in the pancreas following WGP training, all of which display features of trained immunity. It is thus likely the case that in this model there is not a single cell type that is responsible for the slowed tumor growth, but rather the result of a juxtaposition of an increase in pro-inflammatory trained cells and a decrease in the number and function of resident pro-tumorigenic cells. 
It has been noted by several studies that the CCR2+ present within a tumor have protumorigenic roles $[212,213]$. In pancreatic cancer, CCL2 production results in the mobilization of CCR2 $2^{+}$inflammatory monocytes from the bone marrow to the tumor, where they become TAMs [214]. For this reason CCR2 inhibitors used in combination with chemotherapy for PDAC have been tested, and ultimately demonstrated a more favorable response than chemotherapy used alone [215]. In contrast to these studies, here we demonstrate that CCR2+ cells recruited to the tumor have anti-tumor potential due to the fact that they have been trained by WGP. Unlike typical CCR2+ cells recruited to the TME, in this model CCR2+ cells recruited to the tumor site have undergone transcriptional and metabolic reprogramming that allows them to exert anti-tumor properties within the TME. A major downfall of the use of therapeutic use of CCR2 inhibitors is that the depletion of TAMs often results in a compensatory influx of tumor associated neutrophils (TANs) to the TME which themselves have many pro-tumorigenic roles. Therefore instead of using CCR2 inhibitors in the setting of PDAC, it may be more reasonable to consider therapies that reprogram CCR2+ cells that will traffic to the tumor anyways to have antitumor functionalities [216].

We note high expression of MHC II on trained monocyte/macrophages in the pancreas, which likely play a role in tumor antigen processing and communication with T-cells to elicit adaptive immune responses against the tumor. While based on NSG tumor studies, these antitumor mechanisms do not depend on adaptive immune responses, it is conceivable that activated myeloid cells could exert a positive influence on adaptive immune cells to aid in the generation and activation of tumor-specific T-cells and in antitumor adaptive responses. Specifically in the treatment setting where myeloid cells will 
traffic to the pancreas which bears a tumor already, the arrival of activated myeloid cells expressing high levels of MHCII could play an important role in taking up and presenting antigen to T-cells. This could result in an enhanced generation of tumor-specific T-cells that could exert important anti-tumor effects. Accordingly, models where WGP is administered before the tumor is implanted versus after the tumor is implanted may show different types of T-cell responses. While this project focuses primarily on the role of trained innate myeloid cells and their direct anti-tumor effects, more investigations on the consequences of trained innate immunity on anti-tumor T-cell responses is warranted. It may also be important to determine if alterations to the T-cell compartment due to WGP treatment are responsible for the success of combination therapy of WGP and anti-PD-L1 therapy or if these effects are solely due to changes to the myeloid cells.

Another focus of future investigations is the optimization of WGP treatment in terms of dosing and the number of treatments could further extend survival. While we did observe a decrease in tumor size and longer survival due to WGP training, all mice did eventually succumb to the tumor, even in combination with anti-PD-L1 therapy.

Immunosuppressive myeloid cells play a critical role in the creation of the hostile TME in PDAC through the inhibition of T-cell effector function and the promotion of cancer stemness, angiogenesis, stroma deposition, and metastasis formation [217-219]. While it is the case that within the TME tumor secreted factors often reprogram myeloid cells to propagate tumor growth, these cells also harbor the potential for robust anti-tumor responses [220-222]. A conceivable reason why immunotherapy alone has yet to show success in the treatment of PDAC is that these therapies have a primary objective of reactivating tolerogenic $\mathrm{T}$-cells rather than reactivating and reprogramming the 
suppressive myeloid cells that promote them. Accordingly, two therapeutic strategies that could benefit PDAC patients but that have not yet been successfully implemented in a clinical setting are therapeutics that 1) promote the reprogramming of immunosuppressive myeloid cells in the PDAC TME, and 2) actively recruitment anti-tumor myeloid cells to the tumor that can penetrate the desmoplastic stroma. Here, we demonstrate a novel capability to engage both of these anti-tumor mechanisms in the setting of PDAC through the induction of trained innate immunity in the pancreas by particulate $\beta$-glucan. Importantly, we also show that the novel ability to initiate trained immunity in the pancreas potentiates the therapeutic effects of immunotherapy such as anti-PD-L1 immunocheckpoint blockade therapy. Lending further clinical applicability to these findings, we also exhibit that the induction of TI in the adjuvant setting can decrease tumor size and prolong survival. These findings emphasize the potential of using $\beta$-glucan to therapeutically target myeloid cells within the TME and highlight that the induction of peripheral trained immunity in the pancreas may have a consequential role in reprogramming the suppressive TME of PDAC and in extending the life of patients diagnosed with this deadly malignancy. 


\section{SUMMARY AND FUTURE DIRECTIONS}

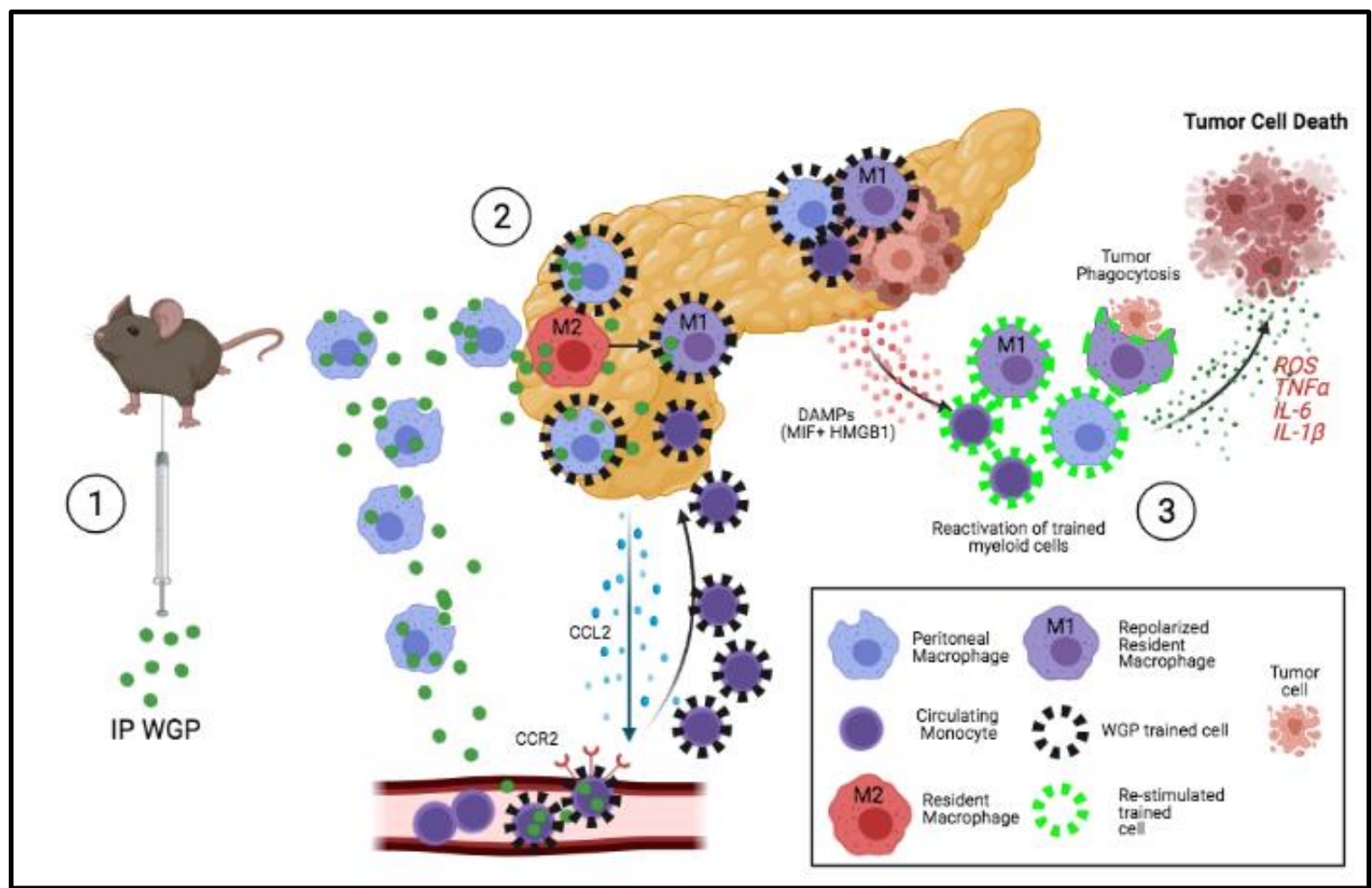

\section{Figure S1: Graphical Abstract}

1) Mice are injected with IP WGP which traffics to the pancreas both in peritoneal macrophages and as naked WGP. 2) M2 resident macrophages in the pancreas are repolarized to an M1 phenotype after WGP trafficking and WGP also impacts peripheral myeloid cells. All cells that come into contact with WGP become trained. The arrival of WGP to the pancreas initiates the production of CCL2 and this signaling recruits CCR2+ trained myeloid cells from the periphery to the pancreas. 3) When trained cells in the pancreas are reactivated by tumor cells and their supernatants, they will produce cytokines and ROS in addition to initiating phagocytosis of tumor cells. Together these mechanisms kill tumor cells and reduce tumor burden. 
The data presented in this dissertation reveal a novel trafficking mechanism of $\beta$ glucan to the pancreas that initiates a CCR2-dependent induction of peripheral trained immunity and reprogramming of M2-like resident macrophages to M1-like. We show that trained cells present in the pancreas following WGP treatment have significant anti-tumor functionality, and that tumor cells and the soluble factors that they secrete can function as a secondary stimulus to reactivate previously trained cells. Through models of orthotopic pancreatic cancer we demonstrate that the induction of peripheral trained immunity in the pancreas leads to an overall reduced tumor burden and longer survival (Fig S1).

We also illustrate that while immunotherapy alone fails to effectively reduce pancreatic tumor burden, treatment with WGP synergizes with anti-PD-L1 mAb therapy to further prolong survival. Finally, we show that WGP effectively reduces tumor burden in the treatment setting. These findings have significant clinical relevance as innovative strategies to effectively target and treat pancreatic cancer are desperately needed.

One potential challenge in the translation of these results to the clinical setting comes from the intraperitoneal route of administration of the WGP. While more invasive than intravenous or oral administration, IP injection is a commonly utilized clinical practice in the treatment of a variety of malignancies including ovarian and mesenteric cancer [223225]. Importantly, the current standard of care for patients with pancreatic cancer often necessitates a surgeon to gain access to the peritoneal cavity providing an opportunity to deliver IP WGP without requiring additional surgical intervention. For example, a patient diagnosed with stage I or II PDAC will usually receive a diagnostic laparoscopy one week prior to final surgical resection followed by adjuvant chemotherapy. In this case, WGP could be administered IP at either the time of the diagnostic laparoscopy or at the time of 
resection. In the case of a patient with locally advanced stage III disease, they will often receive neoadjuvant FOLFIRINOX treatment followed by a diagnostic laparoscopy one week prior to treatment with irreversible electroporation (IRE) [226, 227]. Both the diagnostic laparoscopy and the IRE procedure would provide an opportunity to administer IP WGP. At this time we are in the process of generating additional safety data that would support the creation of a clinical trial. Additionally, we are investigating whether the IP administration of WGP to pigs also results in an influx of trained innate immune cells to the pancreas. Should these studies have positive results, future studies will aim to investigate whether the administration of IP WGP may help in prolonging the survival of human patients diagnosed with pancreatic cancer. These studies would involve investigation into the ideal dose and timing of WGP administration, and would also detail if WGP treatment synergizes with therapies such as IRE or immune-checkpoint blockade therapy.

As checkpoint inhibitor monotherapy has failed to improve patient outcomes in the setting of PDAC, none of these therapies are currently approved for use in PDAC patients [6]. The highly immunosuppressive microenvironment of PDAC poses a significant challenge to the efficacy of immunotherapies, as does the dense desmoplastic stroma and infiltration of immunosuppressive myeloid cells. Our studies highlight that strategies to reprogram the myeloid compartment within PDAC may prove to be effective in unleashing the potential of immunotherapy. Thus, further investigations into the mechanisms that allow WGP to synergize with anti PD-L1 therapy are needed as they may elucidate important underlying mechanisms regarding resistance to immunotherapy that can be applied widely and to other cancer types. 
As discussed earlier, the specific tropism of WGP to the pancreas is also a novel finding and carries with it the potential to load WGP with other therapeutics such as chemotherapy or siRNA and have them directly delivered to the pancreas. More studies are certainly warranted to investigate why WGP shows specific tropism to the pancreas and whether this phenomenon is based on an anatomical or chemical mechanism. Additionally, while the induction of trained immunity in the pancreas was shown to directly have anti-tumor effects, the delivery of cancer therapeutics directly to the pancreas in the setting of PDAC provides a major challenge to its treatment. Further, systemic therapies such as chemotherapy often result in high degrees of toxicity that contribute to patient morbidity and mortality, and often do not effectively target the intended organ because of altered vasculature due to angiogenesis. Hence future studies may focus entirely on how this tropism of WGP to the pancreas can be manipulated to deliver effective doses of antitumor therapeutics. For example, WGP particles loaded with siRNA against PD-L1 could traffic to the pancreas where they not only could induce peripheral trained immunity due to the presence of WGP, but may also be taken up by resident macrophages and DCs in the TME, which would result in decreased PD-L1 expression and enhanced T-cell activation and killing of tumor cells (Fig S2). 


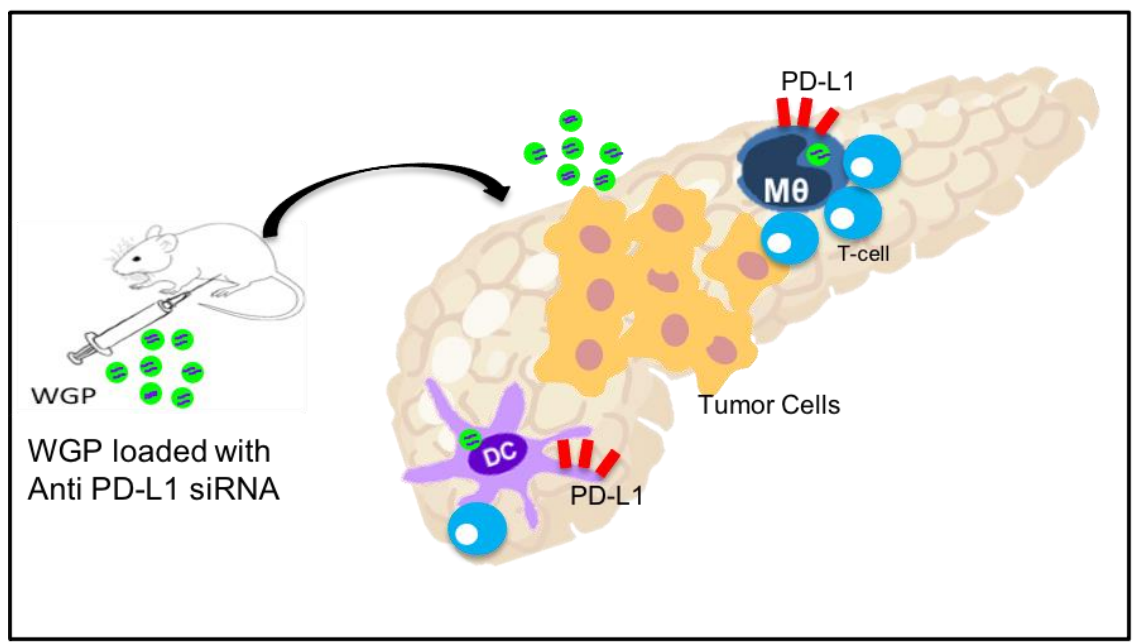

Figure S2: Schematic of the use of WGP particles loaded with anti-tumor agents to target the pancreas

Finally, the induction of peripheral trained immunity in a visceral organ has not been previously reported. Though peripheral trained immunity has been identified to exist, how it is initiated in a specific organ has not been investigated before. This work identifies that WGP arrival to the pancreas stimulates the production of CCL2 which likely plays a major role in recruiting CCR2+ trained myeloid cells to the pancreas that can be restimulated by tumor cells. We also assume that immunosuppressive resident macrophages in the pancreas undergo repolarization due to the arrival of WGP to the pancreas. It is possible that injection of WGP to a specific organ may also result in a similar signaling mechanism that would result in anti-tumor effects, and so future studies are needed to investigate whether the mechanism outlined in this paper is specific to the pancreas or can be deployed to incite anti-tumor effects in other tumor-bearing locations 


\section{METHODS}

\section{Mice:}

Six to eight week-old female wild-type (WT) C57BL/6J mice were purchased from the Jackson Laboratory (Bar Harbor, ME, USA) or bred in the University of Louisville specific pathogen-free (SPF) animal facility. C57BL/6 Dectin-1 knockout (Dectin-1 -/-) mice were described previously [228]. CCR2 global knock-out mice were purchased from Jackson Laboratory. Albino C57BL/6 mice were kindly provided by Dr. Jonathan Warawa at the University of Louisville. NOD/SCID/IL2r $\gamma^{\text {Null }}$ (NSG) mice and B6/SJL-CD45.1 were purchased from the Jackson Laboratory. All mice were at least 6 weeks of age upon use, and all experiments involving animals were performed in compliance with all relevant laws and institutional guidelines provided by the Rodent Rearing Facility (RRF) and approved by the Institutional Animal Care and Use Committee (IACUC) of the University of Louisville.

\section{Preparation of $\beta$-glucan:}

Highly purified particulate $\beta$-glucan in the form of particulate Whole $\beta$-glucan Particles (WGP) isolated from Saccharomyces cerevisiae was provided by Biothera. Before use, WGP was gently sonicated for 15 seconds, 2 times using a Qsonica Q55-110 Q55 Sonicator (Cole-Parmer) to ensure aggregates were broken up.

\section{Preparation and use of DTAF WGP:}


DTAF (Sigma Aldrich) at $2 \mathrm{mg} / \mathrm{mL}$ was mixed with a suspension of $20 \mathrm{mg} / \mathrm{mL}$ WGP in borate buffer ( $\mathrm{pH}$ 10.8). This incubated at room temperature for 8 hours with continuous mixing. Following incubation, the WGP was centrifuged and washed with cold sterile endotoxin-free DPBS (Sigma Aldrich) 5 times or until the supernatant no longer contained visible DTAF. The concentration was adjusted to $10 \mathrm{mg} / \mathrm{mL}$ in the endotoxin-free DPBS for storage. 1mg of the DTAF WGP was injected IP into C57BL/6 and Dectin-1 -/- mice and organs were harvested 3 days later.

\section{Preparation of the ${ }^{89} \mathrm{Zr}-W G P$ :}

WGP (100 mg) was mixed with Deferoxamine-SCN (2.7 mg, in $0.8 \mathrm{ml}$ DMSO) and suspended in $10 \mathrm{ml}$ sodium carbonate buffer $(0.1 \mathrm{M}, \mathrm{pH}$ 9.4) overnight at room temperature in the dark with gentle shaking. The Deferoxamine-labeled WGP was then washed with DI water $(10 \mathrm{X} 40 \mathrm{~mL}$ ), and $30 \mathrm{mg}$ of Deferoxamine-labeled WGP was mixed with $3 \mathrm{mCi}$ of ${ }^{89} \mathrm{Zr}$ oxalate in $2 \mathrm{ml}$ Tris $\bullet \mathrm{HCl}$ buffer $(0.5 \mathrm{M}, \mathrm{pH} 7.5)$, and then incubated at $37{ }^{\circ} \mathrm{C}$ for 60 min with shaking. The ${ }^{89} \mathrm{Zr}$-WGP was then centrifuged and washed with $3 \mathrm{ml}$ of sterile PBS. The radioactivity of ${ }^{89} \mathrm{Zr}$-WGP was measured by a dose calibrator and used for in vitro and in vivo studies.

\section{Biodistribution and PET/CT Scan using ${ }^{89} \mathrm{Zr}$-WGP}

Positron Emission tomography (PET)/computed tomography (CT) imaging was conducted in C57BL/6 and Dectin-1 -/- mice 48 hours after IP injection of $1 \mathrm{mg}$ of pure ${ }^{89} \mathrm{Zr}$-WGP or injection of $1 \times 10^{6}$ peritoneal macrophages that had been co-cultured with $25 \mu \mathrm{g} / \mathrm{ml}$ of

${ }^{89} \mathrm{Zr}$-WGP for 2 hours and then gently washed to remove excess ${ }^{89} \mathrm{Zr}$-WGP. The mice were scanned for 15 min with a Siemens R4 MicroPET and followed by 10 min of CT 
scan. Siemens IAW soft- ware was used for the acquisition and reconstruction of the PET signal, and Siemens IRW software was used for merging and analyzing the imaging data. At the end of the imaging study, mice were euthanized, and organs of interest were harvested. For biodistribution, $50 \mathrm{uL}$ of peripheral blood was collected using a retrobulbar bleeding technique. The brain, heart, lungs, liver, spleen, kidneys, pancreas, large intestine, small intestine, stomach, femur, a piece of skin from the flank of the mice and the rectus femoris muscle were harvested, weighed, and placed in a 2470 Wizard automatic gamma counter (PerkinElmer) in order to measure the radioactivity of each tissue. The CPM values were calculated using Prism software (GraphPad Software, La Jolla, CA).

\section{Pancreatic processing}

Following euthanization, mouse pancreas were harvested and gently cut into smaller pieces using sterile scissors. They were suspended in a $15 \mathrm{~mL}$ tube in complete media (RPMI) with $1 \mathrm{X}$ digestion buffer comprised of 300U/ml collagenase $\mathrm{I}, 60 \mathrm{U} / \mathrm{ml}$ Hyaluronidase, and $80 \mathrm{U} / \mathrm{ml}$ DNase (Sigma). These were placed in a rotating incubator at $37^{\circ} \mathrm{C}$ with $5 \% \mathrm{CO} 2$ for $15-20$ minutes. The digestion buffer was then quenched with ice cold complete RPMI 1640 and washed. Cells were passed through a sterile nylon $40 \mu \mathrm{m}$ basket filter and small undigested pieces of tissue were smashed using a sterile syringe stopper in order to generate a single-cell suspension. If an appreciable number of red blood cells (RBCs) were seen to exist in the sample, RBC lysis was performed by adding $2 \mathrm{~mL}$ of sterile 10x ACK (Thermo Fisher Scientific).

\section{In vivo WGP administration}


Mice were given a single $1 \mathrm{mg}$ intraperitoneal dose of gently sonicated WGP, $3 \mu \mathrm{m}$ polystyrene beads (Sigma Aldrich), or $3 \mu \mathrm{m}$ fluorescent microspheres (Polysciences) (all $1 \mathrm{mg}$ in $200 \mu \mathrm{l}$ of sterile PBS) or $200 \mu \mathrm{l}$ of sterile PBS on day 0 . For typical trained immunity studies, 7 days following the initial IP dose, mice were euthanized using $\mathrm{CO}_{2}$ and the pancreas along with other tissues of interest were removed and processed. For dose titration studies, $.5 \mathrm{mg}, 1 \mathrm{mg}$ and $2 \mathrm{mg}$ of WGP were delivered IP in 200uL of sterile PBS. For time titration studies, mice were injected with $1 \mathrm{mg}$ of WGP and the pancreas was harvested after 24 hours, 48 hours, 3, 7, 10, 16 and 30 days later.

\section{Ex vivo re-stimulation}

In order to assess the trained phenotype of mononuclear cells in mice treated with WGP or PBS ex vivo, after processing the pancreas, pancreatic cell suspensions were plated in 24 well plates and stimulated with LPS (10ng/ml), the supernatant from cultured KPC and Pan02 cells (40\%), and recombinant MIF (rMIF)(10ng/ml). rMIF was a generous gift from Dr. Robert Mitchell at the University of Louisville and was prokaryotically expressed, purified, and re-folded a described previously[229]. Cells were cultured in DMEM, and incubated at $37^{\circ} \mathrm{C}$ with $5 \% \mathrm{CO}_{2}$ for $5-6$ hours in the presence of $1 \mathrm{X}$ brefeldin $\mathrm{A}$ (Biolegend). The cells were then harvested using a cell scrapper, washed, pelleted and then stained for intracellular cytokine expression.

\section{In vitro training and re-stimulation assay}

Peritoneal macrophages or sorted CD11b+ cells from a mouse pancreas were plated in a 24 well plate for 2 hours at $37^{\circ} \mathrm{C}$ and $5 \% \mathrm{CO}_{2}$ to allow for the attachment of cells to the 
plates, after which the floating cells were gently aspirated. Attached cells were gently washed with sterile PBS and then resuspended in $1 \mathrm{~mL}$ of DMEM constituted of $10 \%$ FBS $1 \%$ penicillin/streptomcymin. For the initial training of cells, $25 \mu \mathrm{g} / \mathrm{ml}$ of particulate WGP, $25 \mu \mathrm{g} / \mathrm{ml}$ of $3 \mu \mathrm{m}$ polystyrene microparticle beads (Sigma-Aldrich) or $100 \mathrm{uL}$ of PBS were added to the appropriate well and incubated for 24 hours. After 24 hours, wells were gently washed to remove the initial stimulus and fresh DMEM was added and cells were incubated with $5 \% \mathrm{CO}_{2}$ at $37^{\circ} \mathrm{C}$ for 7 days. After 7 days, the media was aspirated, replaced with fresh media, and cells were re-stimulated with LPS $(100 \mathrm{ng} / \mathrm{mL})$, the supernatant of KPC or Pan02 cells (40\%), or PBS as a control. 24 hours after stimulation, the supernatants were harvested and used in an ELISA for TNF- $\alpha$ and IL-6.

\section{Tumor conditioned media}

$1 \times 10^{6} \mathrm{KPC}$ or Pan02 cells were cultured in a six well plate in $4 \mathrm{~mL}$ of complete DMEM and at $37^{\circ} \mathrm{C}$ and $5 \% \mathrm{CO}_{2}$. After 3 days the supernatants were harvested and stored at $-80^{\circ} \mathrm{C}$ in aliquots for use as tumor-conditioned medium. As a control, the pancreas of a C57BL/6 mouse was processed into a single cell suspension and $1 \times 10^{6}$ of these cells were cultured in a six well plate in $4 \mathrm{~mL}$ of complete DMEM and at $37^{\circ} \mathrm{C}$ and $5 \% \mathrm{CO}_{2}$, and supernatants were also collected after 3 days.

\section{Acquisition of peritoneal macrophages}

Mice were euthanized and $5 \mathrm{~mL}$ of sterile RPMI was injected into the peritoneum using a 25-gague syringe. The abdomen was massaged to liberate the peritoneal macrophages. A small incision was made and a transfer pipette was used to remove the suspension. The 
peritoneal cavity was then washed several times with cold RPMI and cells were pelleted at 1600 RPM.

\section{Confocal Microscopy}

Mice were injected IP with 1mg of DTAF-WGP and 4 hours later mice were euthanized and peritoneal macrophages were harvested. Cells were suspended in $200 \mathrm{uL}$ of sterile PBS and were spun onto a microscope slide using a Shandon CytoSpin3 (Thermo Fisher). Slides were then air dried for 10 min prior to staining. Cells were fixed with $4 \%$ formalin for 30 minutes, washed with PBS, washed again with a $.3 \%$ triton solution, and then incubated with blocking solution (20\% FBS, .3\% TritonX) for 1 hours. Cells were then washed and incubated with F4/80 primary antibody overnight. Wells were washed and cells were incubated with DAPI for 10 minutes. Slides were washed, a cover slip was applied to all samples and images were acquired using a Nikon confocal microscope.

\section{Flow cytometry}

Single-cell suspensions in PBS with $1 \%$ FBS were blocked with murine Fc Block (anti$\mathrm{CD} 16 / \mathrm{CD} 32)$ at $4^{\circ} \mathrm{C}$ for 15 minutes. Fluorochrome labeled antibodies for viability (APC/Cy7) and to surface markers CD45, CD11b, F4/80, Ly6G, Ly6C, MHCII, CD3,CD4,

CD8, NK1.1, Ki67 (Biolegend) and CCR2 (R\&D Systems) were used. After 30 minutes of incubating in the dark at $4^{\circ} \mathrm{C}$, cells were washed with cold PBS, and filtered through a 40 $\mu \mathrm{m}$ mesh filter. The samples were acquired using a FACSCanto II cytometer (BD Biosciences) and analyzed using FlowJo software (Tree Star, Ashland, OR). 


\section{Intracellular staining for expression of cytokines}

Following stimulation, cells were stained for the desired surface markers as described above. The cells were then washed with cold PBS, and 500 $\mu$ l of fixation buffer (Biolegend) was added to the tubes, briefly vortexed and incubated in the dark at room temperature for 20 minutes. $1 \mathrm{ml}$ of Permeabilization buffer (Biolegend) was then added and samples were centrifuged at $1600 \mathrm{rpm}$ for 5 minutes at $4^{\circ} \mathrm{C}$ followed by one more wash using $1 \mathrm{ml}$ of permeabilization buffer. Cells were resuspended in $200 \mu \mathrm{l}$ of permeabilization buffer and cells were stained with antibodies against TNF- $\alpha$, Ki67, Granzyme-B, IL-12, IL-6 and IFN $\gamma$ or the respective isotype control overnight at $4^{\circ} \mathrm{C}$. Cells were then washed, filtered and data was acquired using a flow cytometer.

\section{FACS isolation of CD11b+ macrophages for in vitro training}

The pancreas was processed into a single cell suspension as described above. Cells were washed with $1 \mathrm{~mL}$ of PBS, incubated with Fc block for 10 minutes at $4^{\circ} \mathrm{C}$ followed by staining with viability dye (APC-Cy7), CD45 (PerCPcy5) and CD11b+ (APC) for 30

minutes at $4^{\circ} \mathrm{C}$. Cells were washed with PBS and re-suspended in cold MACS Running Buffer (Miltenyi Biotech). Viability ${ }^{-} \mathrm{CD} 45^{+} \mathrm{CD} 11 \mathrm{~b}^{+}$cells were sorted using a FACS Aria III (Bd Biosciences). Cells were collected in a 50\% FBS,40\% PBS, 10\% HEPES (Corning). After sorting the cells were washed with PBS and then plated for in-vitro training, as described above.

\section{ELISA}


Supernatants from in vitro trained cells along with standards were analyzed using murine TNF- $\alpha$ and IL-6 ELISA kits (BioLegend). The assay was performed per the manufacturer's instructions and all conditions were performed in triplicates. An ELISA kit for r-MIF (R and D systems) was also used according to the manufacturer's instructions in order to quantify r-MIF in Pan02 and KPC tumor conditioned media and the supernatant from cultured untreated pancreatic cells.

\section{qRT-PCR}

After CD11b+ cells had been isolated from the mouse pancreas in WGP treated and untreated mice, cells were saved in TRIzol. RNAs were isolated and reverse transcribed using the TaqMan Reverse Transcription Reagents (qRT-PCR) amplification using the BioRad MyiQ single color RT-PCR detection system. Briefly, cDNA was amplified in a 25uL reaction mixture consisting of SYBR Green PCR super-mix (BioRad), $100 \mathrm{ng}$ of complementary DNA template, and selected primers $(200 \mathrm{nM})$ using the recommended cycling conditions.

\section{$\mathbf{H}+\mathbf{E}$}

7 days following injection with PBS/microparticle beads or WGP, the pancreata were harvested and fixed in $4 \%$ formalin for 1 week followed by embedding in paraffin according to standard procedures. Paraffin embedded tissues were cut into $5 \mathrm{~mm}$ thick sections and strained with hematoxylin and eosin (H \& E) for morphological analysis.

\section{Serum amylase measurement}


Murine serum amylase was measured using the Amylase Activity Assay Kit (Millipore Sigma) and was used according to the manufacturer's instructions. In short, mice were injected with WGP and 7 days later, blood was collected from mice using a retro-bulbar bleeding technique. These samples were used to assay for serum amylase.

\section{In vivo T-cell depletion}

T-cells were depleted using an anti-CD4 mAb alone, anti-CD8 mAb alone or anti-CD4 and anti-CD8 mAbs together. Antibodies were made in-house. In this depletion procedure, mice were injected IP with WGP on day 1 and were also injected IP with $200 \mu \mathrm{g}$ of the mAbs at day 1 and day 4 during the treatment period. Mice were euthanized on day 7. (CD4 clone GK1.5, CD-8 clone 53-6.72). Depletion efficiency was confirmed on day 7.

\section{In vivo NK cell depletion:}

NK cells were depleted through the intraperitoneal injection of $100 \mu \mathrm{g}$ of PK136 mAb (Produced in the laboratory of Dr. Jun Yan at the University of Louisville) on days -1 and 5 during the treatment period. WGP was injected at day 0 , and on day 7 animals were euthanized and the depletion efficiency of NK cells was assessed by staining pancreatic tissues for NK1.1.

\section{Ly6G depletion}

Neutrophils were depleted by injecting $300 \mu \mathrm{g}$ of anti-Ly6G mAb (Bio X Cell) or isotype control Rat IgG2a (Bio X Cell) at day -1, 2 and 6 during the course of treatment. 1mg of WGP was injected IP on day 1. Mice were euthanized on day 7 and the pancreas was 
assessed for efficiency of depletion. In the tumor model mice were injected with $300 \mu \mathrm{g}$ of anti-Ly6G mAb or isotype control Rat IgG2a on day $-2,4,10$ and 16 . Mice were given WGP at day 0 , and were implanted with orthotopic KPC pancreatic tumors on day 7. Mice were euthanized on day 21 and pancreatic tissues were stained with Ly6G to assess granulocyte depletion efficiency at that time.

\section{CyTOF mass cytometry sample preparation}

Mass cytometry antibodies were either purchased from Fluidigm or were created in house by conjugating commercially available purified antibodies to the appropriate metal isotope using the MaxPar X8 Polymer or MCP9 Polymer kits (Fluidigm). Pancreatic samples from three PBS and three 7-day WGP mice were processed into a single cell solution and exvivo stimulation was performed as described above. Cells were gently scraped from the plates using a sterile cell scraper, washed with PBS and placed into a sterile culture tube. $2 \times 10^{6}$ cells per samples were used. Cells were first stained for viability with $5 \mathrm{uM}$ cisplatin (Fluidigm) in serum free RPMI1640 for 5 minutes at RT. Cells were then washed with RPMI1640 containing 10\% FBS for 5 minutes at 300xg. Cells were stained with the surface antibodies for 30 minutes at RT and washed twice with Maxpar Cell staining buffer (Fluidigm). For staining on intracellular cytokines, cells were then fixed with $1 \mathrm{~mL}$ of $1 \mathrm{X}$ Maxpar Fix I buffer for 30minutes at RT and then washed twice with $2 \mathrm{~mL}$ of 1X Maxpar Perm-S buffer for 5 minutes at $800 \mathrm{xg}$. The cytoplasmic/secreted antibody cocktail was then added and incubated with the cells for 30 minutes at RT. Following incubation, cells were washed with $1 \mathrm{~mL}$ of $1 \mathrm{X}$ Maxpar Perm-S buffer for 5 minutes at $800 \mathrm{xg}$ and gently blotted to remove all liquid from the tube. In order to stain for nuclear antigens cells were then 
suspended in $1 \mathrm{~mL}$ of $1 \mathrm{X}$ Maxpar nuclear antigen staining buffer for 30 minutes at RT. The nuclear antigen antibody cocktail was then added and incubated for 30 minutes at RT. Cells were washed with twice for 5 minutes at $800 \mathrm{xg}$ with $2 \mathrm{~mL}$ of Nuclear Antigen Staining Permeability buffer. Finally, cells were fixed with $1.6 \%$ formaldehyde for 10 minutes at RT, then incubated overnight in $125 \mathrm{nM}$ of Intercalator-Iridium (Fluidigm) at $4^{\circ} \mathrm{C}$.

\section{CyTOF data acquisition}

Once cells were ready for acquisition, samples were washed twice with Cell Staining Buffer (Fluidigm) and kept on ice while awaiting acquisition. Directly prior to acquisition, cells were suspended in a 1:9 solution of Cell Acquisition Solution: EQ 4 element calibration beads (Fluidigm). A Helios CyTOF system was used, and following proper startup and tuning procedures, samples were run at a rate of less than or equal to 500 events / second up to 300,000 events. Using the CyTOF software, .FCS files were normalized into .fcs files, and these files were then ready to for data analysis.

\section{CyTOF data analysis}

CyTOF data was analyzed using FlowJo, the CytoBank software package [230], and the CyTOF workflow [231] which includes a suite of packages available in $R$ ( $r$ project.org)[232-235]. For analysis conducted within the CyTOF workflow, FlowJo workspace files exported from flow Workspace and CytoML were used [233].

\section{RNA sequencing: RNA extraction and isolation}


7 days following administration of PBS or WGP IP, pancreata from were harvested, processed into a single cell suspension as described previously, stained for viability, CD45 and CD11b as described previously, and sorted using a FACS Aria III as described previously. Samples were prepared in triplicate for each experimental group. Once these myeloid cells were isolated, cells were washed 2x with ice cold PBS and then lysed with Trizol (Invitrogen). RNAs were extracted using a QIAGEN RNAeasy Kit (QIAGEN). The isolated RNA was checked for integrity using the Agilent Bioanalyzer 2100 system (Agilent Technologies, Santa Clara, CA) and quantified using a Qubit fluorometric assay (Thermo Fisher Scientific, Waltham, MA). Poly-A enriched mRNASeq libraries were prepared following the Universal Plus mRNA-Seq kit standard protocol (Tecan Genomics, Redwood City, CA) using 10 ng of total RNA. All samples were ligated with Illumina adapters and individually barcoded. Absence of adapter dimers and consistent library size of approx. $300 \mathrm{bp}$ was confirmed using the Agilent Bioanalyzer 2100. The library concentration and sequencing behavior was assessed in relation to a standardized spike-in of PhIX using a Nano MiSeq sequencing flow cell from Illumina. $1.8 \mathrm{pM}$ of the pooled libraries with $1 \%$ PhiX spike-in was loaded on one NextSeq 500/550 75 cycle High Output Kit v2 sequencing flow cell and sequenced on the Illumina NextSeq 500 sequencer targeting 60M 1x75bp reads per sample.

\section{RNA sequencing}

Libraries were prepared using the Universal Plus mRNA-seq kit with with NuQuant@ library quantification (NuGen). The six samples were spread across four sequencing lines in one run. The 24 single-end raw sequencing files (.fastq) [236] were downloaded from 
Illumina's BaseSpace [237] (https://basespace.illumina.com/) onto the KBRIN server for analysis. Quality control (QC) of the raw sequence data was performed using FastQC (version 0.10.1) [238]. The sequences were aligned to the mm10 mouse reference genome using STAR (version 2.6) [239], generating alignment files in bam format. Differential expression of ENSEMBL protein-coding transcripts was performed using DESeq2 [240, 241]. Raw counts were obtained from the STAR aligned bam format files using HTSeq (version 0.10.0) [242]. The raw counts were normalized using the Relative Log Expression (RLE) method and then filtered to exclude genes with fewer than 10 counts across the samples [243].

\section{RNA sequencing: Gene Set Enrichment Analysis}

Gene set enrichment analysis (GSEA) was used to further characterize the biology of the genes comprising the WGP vs PBS conditions and their differences [244]. Gene sets were obtained from the Molecular Signatures Database (MSigDB) for Gene Ontology (GO) biological processes and reactome pathways. For each gene set, all tested gene locations in the comparison of WGP vs PBS treated control mice were sorted from highest to lowest significance using $\mathrm{p}$ values. This approach allows highly significant up- and downregulated genes to be included within each gene set, an approach that more accurately reflects the conditions in a biological pathway. For this analysis, a table of the enriched sets is followed by an enrichment plot displaying the profile of the enrichment score (ES) score and position of gene set members on the rank ordered list.

\section{Single-cell sequencing: Isolation of single cells and RNA sequencing}


Live CD45+ cells were sorted from mouse pancreata, washed and resuspended in 1x PBS (calcium and magnesium free) containing $0.04 \%$ BSA. Single cells were captured and barcoded cDNA libraries were constructed using the Chromium Next GEM Single Cell 3' Reagent Kit (v3.1, 10X Genomics) and the Chromium Controller, according to manufacturer's instructions. Libraries were pooled and sequenced using a 28bp x 8bp x $125 \mathrm{bp}$ configuration for read $1 \mathrm{x} i 7$ index $\mathrm{x}$ read 2 on the Illumina NextSeq 500 with the NextSeq 500/550 150 cycle High Output Kit v2.5 (20024907).

\section{Since cell sequencing: Gene expression profiling}

Bcl files were demultiplexed into fastq files using the CellRanger software (10X Genomics, v3.1.0). The total number of sequenced reads was $506,913,062$. The reads were of good quality as determined by FastQC [245]. Gene counts were measured using CellRanger 'count', utilizing the cellranger-mm10-3.0.0 reference genome for mouse. A counts matrix was generated for each individual sample and one aggregated sample with the expected number of cells set at 5,000.

The raw count data determined by CellRanger was used as input to a custom analysis pipeline in R which uses a variety of single-cell analysis tools based on Seurat. The knee plot (Fig S7B) displays a graph showing the ranked UMI counts for each cell barcode for data aggregated across the three groups. Cells above the inflection point represent possible doublets while those below the knee represent background cells. Cell quality control measures were analyzed using Seurat v3 [246], and cell barcodes with the following 
characteristics were removed from the analysis: low counts (possible background cells) with an FDR cutoff of 0.01 from the DropletUtils [247] function 'emptyDrops', high counts (possible doublet cells) with more counts than the knee plot inflection point, mitochondrial content greater than $30 \%$ and ribosomal content greater than $40 \%$. Gene (features) were further filtered to remove retired gene identifiers, and genes that were not expressed in at least two cells. See Figure M1 for the initial and filtered number of cell barcodes and genes.

\begin{tabular}{|c|c|c|c|c|c|}
\hline A Sample & $\begin{array}{c}\text { Initial Cell } \\
\text { Count }\end{array}$ & Knee & Inflection & $\begin{array}{c}\text { Filtered } \\
\text { Genes }\end{array}$ & $\begin{array}{c}\text { Filtered } \\
\text { Cells }\end{array}$ \\
\hline AGGREGATE & 17,370 & 10,162 & 1,013 & 18,301 & 11,132 \\
\hline PBS & 6,448 & 10,419 & 1,199 & 16,802 & 4,213 \\
\hline WGP Day 3 & 5,510 & 12,513 & 600 & 16,340 & 3,757 \\
\hline WGP Day 7 & 5,412 & 8,958 & 1,418 & 16,020 & 3,244 \\
\hline
\end{tabular}

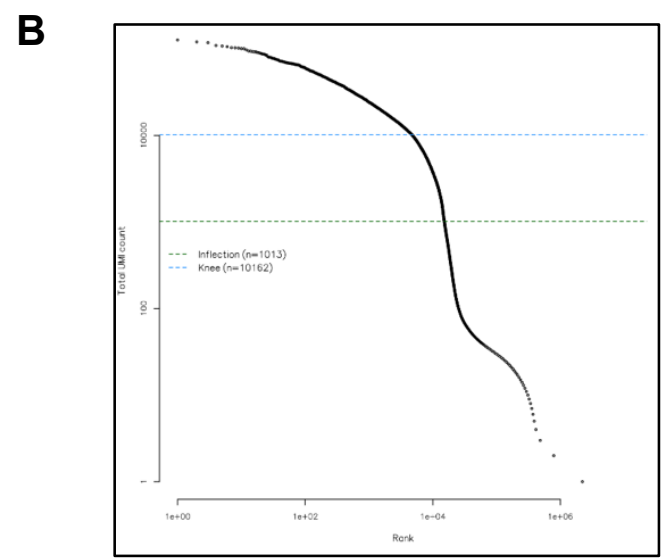

Figure M1: scRNA-seq cell counts and Knee plot

(A) Initial and filtered number of cell barcodes and genes. (B) Ranking of call cells by UMI count of PBS, WGP-3day and WGP-7day, aggregated. Each curve has an associated knee and inflection point, which indicates the likely range of true singlet droplet cells. 
The expression data was normalized using SCTransform [248] where cell cycle genes, ribosomal content, and mitochondrial content were regressed. The cells were then clustered and dimension reduction was performed using UMAP [249]. Initial cluster names were assigned using a modified GSVA $[250,251]$ enrichment score technique. For each of these clusters, the top marker genes were identified. Differentially expressed genes comparing each cluster to every other cluster (all pairwise comparisons) was determined using Seurat [246] and MAST [252].

\section{Identification of clusters generated with scRNA-Seq}

Non myeloid-derived clusters were classified generally as B-cells (MS4A1), plasma cells (SDC1), CD8+ T-cells (CD3e,C8a), CD4+ T-cells (CD3e,CD4), T-regulatory cells (Tregs) (CD3e, CD4, FoxP3), $\gamma \delta$ T-cells (CD3e, TRGC1, TRGC2,IL7Ra) and, type 2 innate immune cells (ILC2s) (Alox5, KLRG1, Ly6a, Pparg,GATA3, IL-5, IL-13, and Rxrg) [253255]. Neutrophils were identified through MMP9, Csf3r, S100A8, S100A8 and ADAM8 expression, though may be underrepresented in these analyses due to their low RNA content and high levels of intrinsic RNases [256]. Conventional dendritic cells (cDCs) were identified through ITGAX and ITGAE expression and plasmacytoid DCs (pDCs) were identified by ITGAX and Siglech.

\section{Phagocytosis assays}

The pancreas of PBS/microparticle injected mice and in-vivo WGP-trained mice were harvested 7 days after injection and processed as described previously into a single cell suspension. $2 \times 10^{6}$ were washed with HEPES dilutes 50x in RPMI 1640 and then incubated 
in $100 \mu \mathrm{L}$ of this solution for 1 hour at $37^{\circ} \mathrm{C}$ in order to activate the cells. The Invitrogen pHrodo $^{\mathrm{TM}}$ Green S. aureus BioParticles ${ }^{\mathrm{TM}}$ Phagocytosis Kit for Flow cytometry (Thermo Fisher Scientific) was used according to the manufacturer's instructions. $100 \mu \mathrm{L}$ of the reconstituted particles or $1 \times 10^{6} \mathrm{GFP}+\mathrm{KPC}$ tumor cells were added to the activated pancreatic cells and incubated for 1 hour at $37^{\circ} \mathrm{C}$. Samples were gently vortexed every 15 minutes. The reaction was stopped by added $1 \mathrm{~mL}$ of cold PBS. Samples were incubated with $\mathrm{Fc}$ Block for 10 minutes at $4^{\circ} \mathrm{C}$, stained for viability, CD45, CD11b and F4/80 for 30 minutes at $4^{\circ} \mathrm{C}$, and then analyzed using a BD FACSCanto. For analysis, after gating on live cells and CD45, cells that fluoresced in the FITC channel were determined to be phagocytic.

\section{Cytotoxicity assay}

The pancreas from WGP and PBS treated mice were harvested and the CD11b+ populations were isolated using magnetic $\mathrm{CD} 11 \mathrm{~b}+$ MicroBeads (Miltenyi Biotec) and an autoMACS Pro Separator (Miltenyi Biotec). Purified CD11b+ cells were then washed and counted, and these were plated at a ratio of 1:20 tumor:effector cells in a 96 well plate. All experimental samples were run in triplicate. After plating the CD11b+ cells, the ROS inhibitor N-acetly-l-cysteine (NAC) (Sigma Aldrich) was added to one set of PBS and WGP derived cells at $1 \mathrm{mM}$ for 1 hour before the addition of 5000 luciferase expressing $\mathrm{KPC}+$ pancreatic tumor cells to all wells. After 24 hours, of co-culture, the plates were centrifuged and $20 \mu \mathrm{L}$ of the supernatant was mixed with $100 \mu \mathrm{L}$ of the Luciferase Assay Reagent (Promega). Luciferase activity measured in the supernatant correlated with tumor cells that had been killed by the effector cells and was measured using a luminometer 
(Femtomaster FB 12, Zylux Corporation). The spontaneous luciferase signal from plated tumor cells was subtracted from the measurement of the supernatant. Luciferase values are represented as Relative Light Units (RLUs).

\section{In vivo tumor models of pancreatic cancer}

A KPC cell line on a C57BL/6 background derived from the $L S L-K_{r a s}{ }^{G 12 D /+}$; LSLTrp5 $3^{R 172 H /+}$; Pdxl-Cre (KPC) mouse model was purchased from Ximbio. These and Pan02 cells which were a generous gift from Dr. Yong Lu at Wake Forest University, were used in an orthotopic model of pancreatic cancer. A KPC line transfected with GFP and luciferase (KPC $\left.{ }^{\mathrm{GFP}+\mathrm{Luc}+}\right)$ were also a generous contribution from Dr. Michael Dwinell at the Medical College of Wisconsin. These cells were used exclusively in albino C57BL/6 mice. For tumor implantation, mice were anesthetized using isoflurane, and the abdomen of the mice were pepped with betadine and draped in a sterile fashion. A $2 \mathrm{~cm}$ midline laparotomy was performed using aseptic technique with sterile instruments. Following laparotomy, the pancreas and spleen were externalized. Tumor cells were suspended in ice cold PBS and mixed in a 1:1 ratio with basement membrane matrix Matrigel (Corning). $0.1 \times 10^{6}$ tumor cells in 50uL of the PBS-matrigel solution were injected into the tail of the pancreas using a 30-guage insulin syringe. The formation of a small bubble indicated successful implantation. The peritoneum was closed using coated polyglycolic acid braided absorbable 5/0 suture and the skin was closed using silk braided nonabsorbable 5/0 suture. (CP Medical). Buprenorphine was administered for pain management up to 72 hours following surgery and mice were monitored. 


\section{In vivo imaging}

Mice implanted orthotopically with GFP+ Luciferase+ KPC tumor cells were injected IP with $150 \mathrm{mg} / \mathrm{kg}$ of body weight at $100 \mu \mathrm{L}$ of XenoLight D-Luciferin-K+ Salt Bioluminescent Substrate (Perkin Elmer). After 10 minutes, mice were anesthetized with isoflurane and placed inside of a Biospace Lab Photon Imager, which is a dedicated low light level in vivo optical modality for bioluminescent and fluorescent imaging. Images of mice were taken and used to measure tumor size and growth.

\section{Generation of BM chimeras}

To generate BM chimera, B6/SJL (CD45.1) mice were treated with IP PBS or WGP and 7 days later the BM was harvested. WT mice (CD45.2) were lethally irradiated (950 cGy) and $2 \times 10^{6} \mathrm{CD} 45+\mathrm{BM}$ cells from the B6/SJL PBS or WGP treated mice were transplanted IV. Six weeks later, the peripheral blood of recipients was analyzed to check the success of the BM transplant, and mice were implanted with $.1 \times 10^{5}$ KPC cells orthotopically. 14 days later mice were euthanized and tumor size was assessed.

\section{Combination therapy with anti PD-L1 mAb}

C57BL/6 mice were treated with WGP at day -7 and implanted with KPC orthotopic pancreatic tumors on day 0 . Mice were treated with $200 \mu \mathrm{g}$ of anti-mouse anti-PD-L1 mAb(Bio X Cell) or Rat IgG2b isotype control (Bio X Cell) at day 3, 7 and 11. Mice were then monitored for survival.

\section{WGP in the treatment setting}


C57BL/6 mice were implanted with KPC orthotopic pancreatic tumors at day 0 and on day 4 and 11 mice were given 1mg of IP WGP or PBS. Mice were then monitored for survival.

\section{Statistical analysis}

Results are represented as mean \pm SEM. Data were analyzed using a two-tailed Student's $\mathrm{t}$ test or Mann-Whitney U-test. Multiple-group comparisons were performed using a oneway or two-way ANOVA followed by Tukey's multiple comparisons test. Correlation analyses were performed using Pearson correlation coefficient (normal distribution). Statistical significance was set at $\mathrm{p}<.05$. All statistical analyses were performed using GraphPad Prism Software Version 9 (GraphPad Inc., La Jolla, CA). 


\section{REFERENCES}

1. ACS. Cancer Facts \& Figures 2020. 2020 [cited 2020; Available from: https://www.cancer.org/content/dam/cancer-org/research/cancer-facts-andstatistics/annual-cancer-facts-and-figures/2020/cancer-facts-and-figures-2020.pdf.

2. Rahib, L., et al., Projecting cancer incidence and deaths to 2030: the unexpected burden of thyroid, liver, and pancreas cancers in the United States. Cancer Res, 2014. 74(11): p. 2913-21.

3. Kommalapati, A., et al., Contemporary Management of Localized Resectable Pancreatic Cancer. Cancers (Basel), 2018. 10(1).

4. Bliss, L.A., et al., Outcomes in operative management of pancreatic cancer. J Surg Oncol, 2014. 110(5): p. 592-8.

5. Schizas, D., et al., Immunotherapy for pancreatic cancer: A 2020 update. Cancer Treat Rev, 2020. 86: p. 102016.

6. Henriksen, A., et al., Checkpoint inhibitors in pancreatic cancer. Cancer Treat Rev, 2019. 78: p. 17-30.

7. Madden, K. and M.K. Kasler, Immune Checkpoint Inhibitors in Lung Cancer and Melanoma. Semin Oncol Nurs, 2019. 35(5): p. 150932. 
8. Kao, H.F. and P.J. Lou, Immune checkpoint inhibitors for head and neck squamous cell carcinoma: Current landscape and future directions. Head Neck, 2019. 41 Suppl 1: p. 4-18.

9. Royal, R.E., et al., Phase 2 trial of single agent Ipilimumab (anti-CTLA-4) for locally advanced or metastatic pancreatic adenocarcinoma. J Immunother, 2010. 33(8): p. 828-33.

10. O'Reilly, E.M., et al., Durvalumab With or Without Tremelimumab for Patients With Metastatic Pancreatic Ductal Adenocarcinoma: A Phase 2 Randomized Clinical Trial. JAMA Oncology, 2019. 5(10): p. 1431-1438.

11. Weiss, G.J., et al., Phase Ib/II study of gemcitabine, nab-paclitaxel, and pembrolizumab in metastatic pancreatic adenocarcinoma. Investigational New Drugs, 2018. 36(1): p. 96-102.

12. Whatcott, C.J., et al., Desmoplasia and chemoresistance in pancreatic cancer, in Pancreatic Cancer and Tumor Microenvironment, P.J. Grippo and H.G. Munshi, Editors. 2012, Transworld Research Network

Copyright () 2012, Transworld Research Network.: Trivandrum (India).

13. Karamitopoulou, E., Tumour microenvironment of pancreatic cancer: immune landscape is dictated by molecular and histopathological features. Br J Cancer, 2019. 121(1): p. 5-14.

14. Daniel, S.K., et al., Hypoxia as a barrier to immunotherapy in pancreatic adenocarcinoma. Clinical and translational medicine, 2019. 8(1): p. 10-10.

15. Geller, A., R. Shrestha, and J. Yan, Yeast-Derived $\beta$-Glucan in Cancer: Novel Uses of a Traditional Therapeutic. Int J Mol Sci, 2019. 20(15). 
16. Rondanelli, M., A. Opizzi, and F. Monteferrario, [The biological activity of betaglucans]. Minerva Med, 2009. 100(3): p. 237-45.

17. Batbayar, S., D.H. Lee, and H.W. Kim, Immunomodulation of Fungal $\beta$-Glucan in Host Defense Signaling by Dectin-1. Biomol Ther (Seoul), 2012. 20(5): p. 433-45.

18. Sletmoen, M. and B.T. Stokke, Higher order structure of (1,3)-beta-D-glucans and its influence on their biological activities and complexation abilities. Biopolymers, 2008. 89(4): p. 310-21.

19. Liu, M., et al., Dectin-1 Activation by a Natural Product $\beta$-Glucan Converts Immunosuppressive Macrophages into an M1-like Phenotype. J Immunol, 2015. 195(10): p. 5055-65.

20. Netea, M.G., J. Quintin, and J.W. van der Meer, Trained immunity: a memory for innate host defense. Cell Host Microbe, 2011. 9(5): p. 355-61.

21. Cheng, S.C., et al., mTOR- and HIF-1 $\alpha$-mediated aerobic glycolysis as metabolic basis for trained immunity. Science, 2014. 345(6204): p. 1250684.

22. Saeed, S., et al., Epigenetic programming of monocyte-to-macrophage differentiation and trained innate immunity. Science, 2014. 345(6204): p. 1251086.

23. Arts, R.J., et al., Glutaminolysis and Fumarate Accumulation Integrate Immunometabolic and Epigenetic Programs in Trained Immunity. Cell Metab, 2016. 24(6): p. 807-819.

24. van der Meer, J.W., et al., Trained immunity: A smart way to enhance innate immune defence. Mol Immunol, 2015. 68(1): p. 40-4. 
25. Namakula, R., et al., Monocytes from neonates and adults have a similar capacity to adapt their cytokine production after previous exposure to BCG and $\beta$-glucan. PLoS One, 2020. 15(2): p. e0229287.

26. Dolasia, K., et al., TLRs/NLRs: Shaping the landscape of host immunity. Int Rev Immunol, 2018. 37(1): p. 3-19.

27. Mogensen, T.H., Pathogen recognition and inflammatory signaling in innate immune defenses. Clin Microbiol Rev, 2009. 22(2): p. 240-73, Table of Contents.

28. Jinushi, M., The role of innate immune signals in antitumor immunity. Oncoimmunology, 2012. 1(2): p. 189-194.

29. Okazaki, T. and T. Honjo, PD-1 and PD-1 ligands: from discovery to clinical application. Int Immunol, 2007. 19(7): p. 813-24.

30. Robert, C., A decade of immune-checkpoint inhibitors in cancer therapy. Nat Commun, 2020. 11(1): p. 3801.

31. Singh, A.K. and J.P. McGuirk, CAR T cells: continuation in a revolution of immunotherapy. Lancet Oncol, 2020. 21(3): p. e168-e178.

32. Gabrielson, A., et al., Intratumoral CD3 and CD8 T-cell Densities Associated with Relapse-Free Survival in HCC. Cancer Immunol Res, 2016. 4(5): p. 419-30.

33. Zakaria, R., et al., T-Cell Densities in Brain Metastases Are Associated with Patient Survival Times and Diffusion Tensor MRI Changes. Cancer Res, 2018. 78(3): p. 610-616.

34. Schulze, A.B., et al., Tumor infiltrating T cells influence prognosis in stage I-III non-small cell lung cancer. J Thorac Dis, 2020. 12(5): p. 1824-1842. 
35. Chen, Y., et al., Tumor-associated macrophages: an accomplice in solid tumor progression. J Biomed Sci, 2019. 26(1): p. 78.

36. Gabrilovich, D.I. and S. Nagaraj, Myeloid-derived suppressor cells as regulators of the immune system. Nat Rev Immunol, 2009. 9(3): p. 162-74.

37. Gordon, S. and P.R. Taylor, Monocyte and macrophage heterogeneity. Nat Rev Immunol, 2005. 5(12): p. 953-64.

38. Yosef, N., et al., The phenotypic and functional properties of mouse yolk-sacderived embryonic macrophages. Dev Biol, 2018. 442(1): p. 138-154.

39. Murdoch, C., et al., The role of myeloid cells in the promotion of tumour angiogenesis. Nat Rev Cancer, 2008. 8(8): p. 618-31.

40. Qian, B.Z. and J.W. Pollard, Macrophage diversity enhances tumor progression and metastasis. Cell, 2010. 141(1): p. 39-51.

41. Dong, P., et al., CD86 $/ C D 206^{+}$, Diametrically Polarized Tumor-Associated Macrophages, Predict Hepatocellular Carcinoma Patient Prognosis. Int J Mol Sci, 2016. 17(3): p. 320 .

42. Klingen, T.A., et al., Tumor-associated macrophages are strongly related to vascular invasion, non-luminal subtypes, and interval breast cancer. Hum Pathol, 2017. 69: p. 72-80.

43. Jeong, H., et al., Tumor-Associated Macrophages as Potential Prognostic Biomarkers of Invasive Breast Cancer. J Breast Cancer, 2019. 22(1): p. 38-51.

44. Petrillo, M., et al., Polarisation of Tumor-Associated Macrophages toward M2 Phenotype Correlates with Poor Response to Chemoradiation and Reduced 
Survival in Patients with Locally Advanced Cervical Cancer. PLoS One, 2015. 10(9): p. e 0136654.

45. van Dalen, F.J., et al., Molecular Repolarisation of Tumour-Associated Macrophages. Molecules, 2018. 24(1).

46. Chen, X.J., et al., The role of the hypoxia-Nrp-1 axis in the activation of M2-like tumor-associated macrophages in the tumor microenvironment of cervical cancer. Mol Carcinog, 2019. 58(3): p. 388-397.

47. Movahedi, K. and J.A. Van Ginderachter, The Ontogeny and Microenvironmental Regulation of Tumor-Associated Macrophages. Antioxid Redox Signal, 2016. 25(14): p. 775-791.

48. Liu, J., et al., Tumor-associated macrophages recruit CCR6+ regulatory $T$ cells and promote the development of colorectal cancer via enhancing CCL2O production in mice. PLoS One, 2011. 6(4): p. e19495.

49. Ngambenjawong, C., H.H. Gustafson, and S.H. Pun, Progress in tumor-associated macrophage (TAM)-targeted therapeutics. Adv Drug Deliv Rev, 2017. 114: p. 206221.

50. Vasiljeva, O., et al., Tumor cell-derived and macrophage-derived cathepsin B promotes progression and lung metastasis of mammary cancer. Cancer Res, 2006. 66(10): p. 5242-50.

51. Chen, J., et al., CCL18 from tumor-associated macrophages promotes breast cancer metastasis via PITPNM3. Cancer Cell, 2011. 19(4): p. 541-55. 
52. $\mathrm{Xu}, \mathrm{J}$., et al., CSF1R signaling blockade stanches tumor-infiltrating myeloid cells and improves the efficacy of radiotherapy in prostate cancer. Cancer Res, 2013. 73(9): p. 2782-94.

53. Kono, K., K. Mimura, and R. Kiessling, Immunogenic tumor cell death induced by chemoradiotherapy: molecular mechanisms and a clinical translation. Cell Death Dis, 2013. 4(6): p. e688.

54. Shiao, S.L., et al., TH2-Polarized CD4(+) T Cells and Macrophages Limit Efficacy of Radiotherapy. Cancer Immunol Res, 2015. 3(5): p. 518-25.

55. Kong, L., et al., Deletion of interleukin-6 in monocytes/macrophages suppresses the initiation of hepatocellular carcinoma in mice. J Exp Clin Cancer Res, 2016. 35(1): p. 131.

56. Law, A.M.K., F. Valdes-Mora, and D. Gallego-Ortega, Myeloid-Derived Suppressor Cells as a Therapeutic Target for Cancer. Cells, 2020. 9(3).

57. Gabrilovich, D.I., S. Ostrand-Rosenberg, and V. Bronte, Coordinated regulation of myeloid cells by tumours. Nat Rev Immunol, 2012. 12(4): p. 253-68.

58. Huang, B., et al., CCL2/CCR2 pathway mediates recruitment of myeloid suppressor cells to cancers. Cancer Lett, 2007. 252(1): p. 86-92.

59. Obermajer, N., et al., PGE(2)-driven induction and maintenance of cancerassociated myeloid-derived suppressor cells. Immunol Invest, 2012. 41(6-7): p. 635-57.

60. Sade-Feldman, M., et al., Tumor necrosis factor- $\alpha$ blocks differentiation and enhances suppressive activity of immature myeloid cells during chronic inflammation. Immunity, 2013. 38(3): p. 541-54. 
61. Meyer, C., et al., Chronic inflammation promotes myeloid-derived suppressor cell activation blocking antitumor immunity in transgenic mouse melanoma model. Proc Natl Acad Sci U S A, 2011. 108(41): p. 17111-6.

62. Ezernitchi, A.V., et al., TCR zeta down-regulation under chronic inflammation is mediated by myeloid suppressor cells differentially distributed between various lymphatic organs. J Immunol, 2006. 177(7): p. 4763-72.

63. Vaknin, I., et al., A common pathway mediated through Toll-like receptors leads to T- and natural killer-cell immunosuppression. Blood, 2008. 111(3): p. 1437-47.

64. Almand, B., et al., Clinical significance of defective dendritic cell differentiation in cancer. Clin Cancer Res, 2000. 6(5): p. 1755-66.

65. Huang, B., et al., Gr-1+CD115+ immature myeloid suppressor cells mediate the development of tumor-induced $T$ regulatory cells and T-cell anergy in tumorbearing host. Cancer Res, 2006. 66(2): p. 1123-31.

66. Meirow, Y., J. Kanterman, and M. Baniyash, Paving the Road to Tumor Development and Spreading: Myeloid-Derived Suppressor Cells are Ruling the Fate. Front Immunol, 2015. 6: p. 523.

67. Ai, L., et al., Prognostic role of myeloid-derived suppressor cells in cancers: a systematic review and meta-analysis. BMC Cancer, 2018. 18(1): p. 1220.

68. Zhang, X., et al., The prognostic value of myeloid derived suppressor cell level in hepatocellular carcinoma: A systematic review and meta-analysis. PLoS One, 2019. 14(12): p. e0225327. 
69. Okła, K., et al., Clinical Relevance and Immunosuppressive Pattern of Circulating and Infiltrating Subsets of Myeloid-Derived Suppressor Cells (MDSCs) in Epithelial Ovarian Cancer. Front Immunol, 2019. 10: p. 691.

70. Takahashi, K., F. Yamamura, and M. Naito, Differentiation, maturation, and proliferation of macrophages in the mouse yolk sac: a light-microscopic, enzymecytochemical, immunohistochemical, and ultrastructural study. J Leukoc Biol, 1989. 45(2): p. 87-96.

71. Perdiguero, E.G. and F. Geissmann, The development and maintenance of resident macrophages. Nat Immunol, 2016. 17(1): p. 2-8.

72. Alliot, F., I. Godin, and B. Pessac, Microglia derive from progenitors, originating from the yolk sac, and which proliferate in the brain. Brain Res Dev Brain Res, 1999. 117(2): p. 145-52.

73. Chorro, L., et al., Langerhans cell (LC) proliferation mediates neonatal development, homeostasis, and inflammation-associated expansion of the epidermal LC network. J Exp Med, 2009. 206(13): p. 3089-100.

74. Bain, C.C., et al., Constant replenishment from circulating monocytes maintains the macrophage pool in the intestine of adult mice. Nat Immunol, 2014. 15(10): p. 929-937.

75. Bouwens, L., et al., Quantitation, tissue distribution and proliferation kinetics of Kupffer cells in normal rat liver. Hepatology, 1986. 6(4): p. 718-22.

76. Davies, L.C., et al., A quantifiable proliferative burst of tissue macrophages restores homeostatic macrophage populations after acute inflammation. Eur J Immunol, 2011. 41(8): p. 2155-64. 
77. Loyher, P.L., et al., Macrophages of distinct origins contribute to tumor development in the lung. J Exp Med, 2018. 215(10): p. 2536-2553.

78. Zhu, Y., et al., Tissue-Resident Macrophages in Pancreatic Ductal Adenocarcinoma Originate from Embryonic Hematopoiesis and Promote Tumor Progression. Immunity, 2017. 47(2): p. 323-338.e6.

79. Etzerodt, A., et al., Tissue-resident macrophages in omentum promote metastatic spread of ovarian cancer. J Exp Med, 2020. 217(4).

80. $\mathrm{Wu}, \mathrm{S} . \mathrm{Y}$. and $\mathrm{K}$. Watabe, The roles of microglia/macrophages in tumor progression of brain cancer and metastatic disease. Front Biosci (Landmark Ed), 2017. 22: p. 1805-1829.

81. Watters, J.J., J.M. Schartner, and B. Badie, Microglia function in brain tumors. J Neurosci Res, 2005. 81(3): p. 447-55.

82. Wei, J., K. Gabrusiewicz, and A. Heimberger, The controversial role of microglia in malignant gliomas. Clin Dev Immunol, 2013. 2013: p. 285246.

83. Müller, A., et al., Resident microglia, and not peripheral macrophages, are the main source of brain tumor mononuclear cells. Int J Cancer, 2015. 137(2): p. 27888.

84. Costa-Silva, B., et al., Pancreatic cancer exosomes initiate pre-metastatic niche formation in the liver. Nat Cell Biol, 2015. 17(6): p. 816-26.

85. Nielsen, S.R. and M.C. Schmid, Macrophages as Key Drivers of Cancer Progression and Metastasis. Mediators Inflamm, 2017. 2017: p. 9624760. 
86. Sarantis, P., et al., Pancreatic ductal adenocarcinoma: Treatment hurdles, tumor microenvironment and immunotherapy. World J Gastrointest Oncol, 2020. 12(2): p. $173-181$.

87. Kleeff, J., et al., Pancreatic cancer. Nat Rev Dis Primers, 2016. 2: p. 16022.

88. Alrawashdeh, W., et al., Perineural invasion in pancreatic cancer: proteomic analysis and in vitro modelling. Mol Oncol, 2019. 13(5): p. 1075-1091.

89. Ozawa, F., et al., Treatment of pancreatic cancer: the role of surgery. Dig Dis, 2001. 19(1): p. 47-56.

90. Michalski, C.W., J. Weitz, and M.W. Büchler, Surgery insight: surgical management of pancreatic cancer. Nat Clin Pract Oncol, 2007. 4(9): p. 526-35.

91. Weledji, E.P., et al., How Grim is Pancreatic Cancer? Oncol Rev, 2016. 10(1): p. 294.

92. Brunner, M., et al., Current Clinical Strategies of Pancreatic Cancer Treatment and Open Molecular Questions. Int J Mol Sci, 2019. 20(18).

93. Conroy, T., et al., FOLFIRINOX or Gemcitabine as Adjuvant Therapy for Pancreatic Cancer. N Engl J Med, 2018. 379(25): p. 2395-2406.

94. Vaccaro, V., I. Sperduti, and M. Milella, FOLFIRINOX versus gemcitabine for metastatic pancreatic cancer. N Engl J Med, 2011. 365(8): p. 768-9; author reply 769.

95. Grant, T.J., K. Hua, and A. Singh, Molecular Pathogenesis of Pancreatic Cancer. Prog Mol Biol Transl Sci, 2016. 144: p. 241-275. 
96. Cheng, X., G. Zhao, and Y. Zhao, Combination Immunotherapy Approaches for Pancreatic Cancer Treatment. Can J Gastroenterol Hepatol, 2018. 2018: p. 6240467.

97. Vonlaufen, A., et al., Pancreatic stellate cells: partners in crime with pancreatic cancer cells. Cancer Res, 2008. 68(7): p. 2085-93.

98. Ho, W.J., E.M. Jaffee, and L. Zheng, The tumour microenvironment in pancreatic cancer - clinical challenges and opportunities. Nat Rev Clin Oncol, 2020. 17(9): p. $527-540$.

99. Apte, M.V., et al., Pancreatic stellate cells are activated by proinflammatory cytokines: implications for pancreatic fibrogenesis. Gut, 1999. 44(4): p. 534-41.

100. Hiraoka, N., et al., Prevalence of FOXP3+ regulatory T cells increases during the progression of pancreatic ductal adenocarcinoma and its premalignant lesions. Clin Cancer Res, 2006. 12(18): p. 5423-34.

101. Pergamo, M. and G. Miller, Myeloid-derived suppressor cells and their role in pancreatic cancer. Cancer Gene Ther, 2017. 24(3): p. 100-105.

102. Yamamoto, T., et al., Circulating CD4+CD25+ regulatory $T$ cells in patients with pancreatic cancer. Pancreas, 2012. 41(3): p. 409-15.

103. Longo, V., et al., Angiogenesis in pancreatic ductal adenocarcinoma: A controversial issue. Oncotarget, 2016. 7(36): p. 58649-58658.

104. Markowitz, J., et al., Patients with pancreatic adenocarcinoma exhibit elevated levels of myeloid-derived suppressor cells upon progression of disease. Cancer Immunol Immunother, 2015. 64(2): p. 149-59. 
105. Bayne, L.J., et al., Tumor-derived granulocyte-macrophage colony-stimulating factor regulates myeloid inflammation and $T$ cell immunity in pancreatic cancer. Cancer Cell, 2012. 21(6): p. 822-35.

106. Trovato, R., et al., Immunosuppression by monocytic myeloid-derived suppressor cells in patients with pancreatic ductal carcinoma is orchestrated by STAT3. J Immunother Cancer, 2019. 7(1): p. 255.

107. Corzo, C.A., et al., HIF-1 $\alpha$ regulates function and differentiation of myeloidderived suppressor cells in the tumor microenvironment. J Exp Med, 2010. 207(11): p. 2439-53.

108. Yoshikawa, K., et al., Impact of tumor-associated macrophages on invasive ductal carcinoma of the pancreas head. Cancer Sci, 2012. 103(11): p. 2012-20.

109. Weizman, N., et al., Macrophages mediate gemcitabine resistance of pancreatic adenocarcinoma by upregulating cytidine deaminase. Oncogene, 2014. 33(29): p. 3812-9.

110. Engblom, C., C. Pfirschke, and M.J. Pittet, The role of myeloid cells in cancer therapies. Nat Rev Cancer, 2016. 16(7): p. 447-62.

111. Mitchem, J.B., et al., Targeting tumor-infiltrating macrophages decreases tumorinitiating cells, relieves immunosuppression, and improves chemotherapeutic responses. Cancer Res, 2013. 73(3): p. 1128-41.

112. Zhu, Y., et al., CSF1/CSF1R blockade reprograms tumor-infiltrating macrophages and improves response to T-cell checkpoint immunotherapy in pancreatic cancer models. Cancer Res, 2014. 74(18): p. 5057-69. 
113. Purvis, A. and A. Hector, Getting the measure of biodiversity. Nature, 2000. 405(6783): p. 212-9.

114. Flajnik, M.F., A cold-blooded view of adaptive immunity. Nat Rev Immunol, 2018. 18(7): p. 438-453.

115. Geller, A. and J. Yan, Could the Induction of Trained Immunity by $\beta$-Glucan Serve as a Defense Against COVID-19? Front Immunol, 2020. 11: p. 1782.

116. Naeslund, C., Expérience de vaccination par le BCG dans la province du Norrbotten. Revue de la Tuberculose, 1931. 12: p. 617-636.

117. Netea, M.G. and J.W. van der Meer, Trained Immunity: An Ancient Way of Remembering. Cell Host Microbe, 2017. 21(3): p. 297-300.

118. Di Luzio, N.R. and D.L. Williams, Protective effect of glucan against systemic Staphylococcus aureus septicemia in normal and leukemic mice. Infect Immun, 1978. 20(3): p. 804-10.

119. Tribouley, J., J. Tribouley-Duret, and M. Appriou, [Effect of Bacillus Callmette Guerin (BCG) on the receptivity of nude mice to Schistosoma mansoni]. C R Seances Soc Biol Fil, 1978. 172(5): p. 902-4.

120. Bistoni, F., et al., Immunomodulation by a low-virulence, agerminative variant of Candida albicans. Further evidence for macrophage activation as one of the effector mechanisms of nonspecific anti-infectious protection. J Med Vet Mycol, 1988. 26(5): p. 285-99.

121. Vecchiarelli, A., et al., Protective immunity induced by low-virulence Candida albicans: cytokine production in the development of the anti-infectious state. Cell Immunol, 1989. 124(2): p. 334-44. 
122. van 't Wout, J.W., R. Poell, and R. van Furth, The role of BCG/PPD-activated macrophages in resistance against systemic candidiasis in mice. Scand J Immunol, 1992. 36(5): p. 713-9.

123. Quintin, J., et al., Candida albicans infection affords protection against reinfection via functional reprogramming of monocytes. Cell Host Microbe, 2012. 12(2): p. 223-32.

124. Moorlag, S., et al., $\beta$-Glucan Induces Protective Trained Immunity against Mycobacterium tuberculosis Infection: A Key Role for IL-1. Cell Rep, 2020. 31(7): p. 107634.

125. Dos Santos, J.C., et al., $\beta$-Glucan-Induced Trained Immunity Protects against Leishmania braziliensis Infection: a Crucial Role for IL-32. Cell Rep, 2019. 28(10): p. 2659-2672.e6.

126. Vetvicka, V. and J. Vetvickova, Glucan supplementation enhances the immune response against an influenza challenge in mice. Ann Transl Med, 2015. 3(2): p. 22.

127. Netea, M.G., et al., Trained immunity: A program of innate immune memory in health and disease. Science (New York, N.Y.), 2016. 352(6284): p. aaf1098aaf1098.

128. Kleinnijenhuis, J., et al., BCG-induced trained immunity in NK cells: Role for nonspecific protection to infection. Clin Immunol, 2014. 155(2): p. 213-9.

129. Hole, C.R., et al., Induction of memory-like dendritic cell responses in vivo. Nat Commun, 2019. 10(1): p. 2955. 
130. Kalafati, L., et al., Innate Immune Training of Granulopoiesis Promotes Anti-tumor Activity. Cell, 2020. 183(3): p. 771-785.e12.

131. Mitroulis, I., et al., Modulation of Myelopoiesis Progenitors Is an Integral Component of Trained Immunity. Cell, 2018. 172(1-2): p. 147-161.e12.

132. Netea, M.G., et al., Trained immunity: A program of innate immune memory in health and disease. Science, 2016. 352(6284): p. aaf1098.

133. Cramer, D.E., et al., Mobilization of hematopoietic progenitor cells by yeastderived beta-glucan requires activation of matrix metalloproteinase-9. Stem Cells, 2008. 26(5): p. 1231-40.

134. Saccani, S., S. Pantano, and G. Natoli, Two waves of nuclear factor kappaB recruitment to target promoters. J Exp Med, 2001. 193(12): p. 1351-9.

135. Smale, S.T., A. Tarakhovsky, and G. Natoli, Chromatin contributions to the regulation of innate immunity. Annu Rev Immunol, 2014. 32: p. 489-511.

136. Smale, S.T. and G. Natoli, Transcriptional control of inflammatory responses. Cold Spring Harb Perspect Biol, 2014. 6(11): p. a016261.

137. Ramirez-Carrozzi, V.R., et al., A unifying model for the selective regulation of inducible transcription by CpG islands and nucleosome remodeling. Cell, 2009. 138(1): p. 114-28.

138. Ramirez-Carrozzi, V.R., et al., Selective and antagonistic functions of SWI/SNF and Mi-2beta nucleosome remodeling complexes during an inflammatory response. Genes Dev, 2006. 20(3): p. 282-96.

139. van der Heijden, C., et al., Epigenetics and Trained Immunity. Antioxid Redox Signal, 2018. 29(11): p. 1023-1040. 
140. Netea, M.G., et al., An integrated model of the recognition of Candida albicans by the innate immune system. Nat Rev Microbiol, 2008. 6(1): p. 67-78.

141. Drummond, R.A., et al., The role of Syk/CARD9 coupled C-type lectins in antifungal immunity. Eur J Immunol, 2011. 41(2): p. 276-81.

142. Gringhuis, S.I., et al., Dectin-1 directs $T$ helper cell differentiation by controlling noncanonical NF-kappaB activation through Raf-1 and Syk. Nat Immunol, 2009. 10(2): p. 203-13.

143. Geller, A., R. Shrestha, and J. Yan, Yeast-Derived $\beta$-Glucan in Cancer: Novel Uses of a Traditional Therapeutic. International journal of molecular sciences, 2019. 20(15): p. 3618 .

144. Fanucchi, S., et al., Immune genes are primed for robust transcription by proximal long noncoding RNAs located in nuclear compartments. Nat Genet, 2019. 51(1): p. 138-150.

145. Lauberth, S.M., et al., H3K4me3 interactions with TAF3 regulate preinitiation complex assembly and selective gene activation. Cell, 2013. 152(5): p. 1021-36.

146. Donohoe, D.R. and S.J. Bultman, Metaboloepigenetics: interrelationships between energy metabolism and epigenetic control of gene expression. J Cell Physiol, 2012. 227(9): p. 3169-77.

147. Michelucci, A., et al., Immune-responsive gene 1 protein links metabolism to immunity by catalyzing itaconic acid production. Proc Natl Acad Sci U S A, 2013. 110(19): p. 7820-5. 
148. Priem, B., et al., Trained Immunity-Promoting Nanobiologic Therapy Suppresses Tumor Growth and Potentiates Checkpoint Inhibition. Cell, 2020. 183(3): p. 786801.e19.

149. Flemming, A., Nanobiologic trains innate immunity for anticancer responses. Nat Rev Immunol, 2020. 20(12): p. 718-719.

150. Killion, J.J., R. Radinsky, and I.J. Fidler, Orthotopic models are necessary to predict therapy of transplantable tumors in mice. Cancer Metastasis Rev, 1998. 17(3): p. 279-84.

151. Naito, S., A.C. von Eschenbach, and I.J. Fidler, Different growth pattern and biologic behavior of human renal cell carcinoma implanted into different organs of nude mice. J Natl Cancer Inst, 1987. 78(2): p. 377-85.

152. Morikawa, K., et al., Influence of organ environment on the growth, selection, and metastasis of human colon carcinoma cells in nude mice. Cancer Res, 1988. 48(23): p. 6863-71.

153. Hoffman, R.M., The multiple uses of fluorescent proteins to visualize cancer in vivo. Nat Rev Cancer, 2005. 5(10): p. 796-806.

154. Fung, A.S., et al., The effect of chemotherapeutic agents on tumor vasculature in subcutaneous and orthotopic human tumor xenografts. BMC Cancer, 2015. 15: p. 112.

155. Buqué, A. and L. Galluzzi, Modeling Tumor Immunology and Immunotherapy in Mice. Trends Cancer, 2018. 4(9): p. 599-601.

156. Guerin, M.V., et al., Preclinical murine tumor models: a structural and functional perspective. Elife, 2020. 9. 
157. Stier, H., V. Ebbeskotte, and J. Gruenwald, Immune-modulatory effects of dietary Yeast Beta-1,3/1,6-D-glucan. Nutr J, 2014. 13: p. 38.

158. Volman, J.J., J.D. Ramakers, and J. Plat, Dietary modulation of immune function by beta-glucans. Physiol Behav, 2008. 94(2): p. 276-84.

159. Suzuki, I., et al., Immunomodulation by orally administered beta-glucan in mice. Int J Immunopharmacol, 1989. 11(7): p. 761-9.

160. de Graaff, P., et al., Consumption of $\beta$-glucans to spice up $T$ cell treatment of tumors: a review. Expert Opin Biol Ther, 2018. 18(10): p. 1023-1040.

161. Harnack, U., et al., Comparison of the effect of orally administered soluble beta(1-3),(1-6)-D-glucan and of G-CSF on the recovery of murine hematopoiesis. In Vivo, 2010. 24(1): p. 59-63.

162. Zheng, X., et al., Uptake of intraperitoneally administrated triple helical $\beta$-glucan for antitumor activity in murine tumor models. J Mater Chem B, 2017. 5(47): p. 9337-9345.

163. Shrivastava, P. and M. Bhatia, Essential role of monocytes and macrophages in the progression of acute pancreatitis. World J Gastroenterol, 2010. 16(32): p. 39954002.

164. Wang, J. and P. Kubes, A Reservoir of Mature Cavity Macrophages that Can Rapidly Invade Visceral Organs to Affect Tissue Repair. Cell, 2016. 165(3): p. 66878.

165. Weisser, S.B., N. van Rooijen, and L.M. Sly, Depletion and reconstitution of macrophages in mice. J Vis Exp, 2012(66): p. 4105. 
166. Soto, E.R., et al., Glucan particles for macrophage targeted delivery of nanoparticles. J Drug Deliv, 2012. 2012: p. 143524.

167. Hwang, J., et al., Synthesis of Beta-glucan Nanoparticles for the Delivery of Single Strand DNA. Biotechnology and Bioprocess Engineering, 2018. 23(2): p. 144-149.

168. Wang, H., et al., $\beta$-Glucan as an immune activator and a carrier in the construction of a synthetic MUC1 vaccine. Chem Commun (Camb), 2018. 55(2): p. 253-256.

169. Berner, V.K., et al., Microparticulate $\beta$-glucan vaccine conjugates phagocytized by dendritic cells activate both naïve CD4 and CD8 T cells in vitro. Cell Immunol, 2015. 298(1-2): p. 104-14.

170. Bach, J.P., et al., Role of MIF in inflammation and tumorigenesis. Oncology, 2008. 75(3-4): p. 127-33.

171. Zhang, M., et al., Non-viral nanoparticle delivers small interfering RNA to macrophages in vitro and in vivo. PLoS One, 2015. 10(3): p. e0118472.

172. Negi, S., et al., Potential Role of Gut Microbiota in Induction and Regulation of Innate Immune Memory. Front Immunol, 2019. 10: p. 2441.

173. McCoy, K.D., R. Burkhard, and M.B. Geuking, The microbiome and immune memory formation. Immunol Cell Biol, 2019. 97(7): p. 625-635.

174. Laukens, D., et al., Heterogeneity of the gut microbiome in mice: guidelines for optimizing experimental design. FEMS Microbiol Rev, 2016. 40(1): p. 117-32.

175. Rausch, P., et al., Analysis of factors contributing to variation in the C57BL/6J fecal microbiota across German animal facilities. Int J Med Microbiol, 2016. 306(5): p. 343-355. 
176. Ericsson, A.C., et al., Effects of vendor and genetic background on the composition of the fecal microbiota of inbred mice. PLoS One, 2015. 10(2): p. e0116704.

177. Kierdorf, K., et al., Development and function of tissue resident macrophages in mice. Semin Immunol, 2015. 27(6): p. 369-78.

178. Calderon, B., et al., The pancreas anatomy conditions the origin and properties of resident macrophages. J Exp Med, 2015. 212(10): p. 1497-512.

179. Kim, H., The transcription factor MafB promotes anti-inflammatory M2 polarization and cholesterol efflux in macrophages. Sci Rep, 2017. 7(1): p. 7591.

180. Kim, S.Y., et al., Anti-inflammatory effects of apocynin, an inhibitor of NADPH oxidase, in airway inflammation. Immunol Cell Biol, 2012. 90(4): p. 441-8.

181. Liu, M., et al., Transcription factor c-Maf is a checkpoint that programs macrophages in lung cancer. J Clin Invest, 2020. 130(4): p. 2081-2096.

182. Zimmerman, K.A., et al., Single-Cell RNA Sequencing Identifies Candidate Renal Resident Macrophage Gene Expression Signatures across Species. J Am Soc Nephrol, 2019. 30(5): p. 767-781.

183. Wang, A.W., et al., The Greater Omentum-A Vibrant and Enigmatic Immunologic Organ Involved in Injury and Infection Resolution. Shock, 2020. 53(4): p. 384-390.

184. Carlow, D.A., M.R. Gold, and H.J. Ziltener, Lymphocytes in the peritoneum home to the omentum and are activated by resident dendritic cells. J Immunol, 2009. 183(2): p. 1155-65.

185. Rehermann, B., Mature peritoneal macrophages take an avascular route into the injured liver and promote tissue repair. Hepatology, 2017. 65(1): p. 376-379. 
186. Cruz, A.F., R. Rohban, and F. Esni, Macrophages in the pancreas: Villains by circumstances, not necessarily by actions. Immun Inflamm Dis, 2020. 8(4): p. 807824.

187. Lech, M. and H.J. Anders, Macrophages and fibrosis: How resident and infiltrating mononuclear phagocytes orchestrate all phases of tissue injury and repair. Biochim Biophys Acta, 2013. 1832(7): p. 989-97.

188. Netea, M.G., et al., Defining trained immunity and its role in health and disease. Nat Rev Immunol, 2020. 20(6): p. 375-388.

189. Priem, B., et al., Trained Immunity-Promoting Nanobiologic Therapy Suppresses Tumor Growth and Potentiates Checkpoint Inhibition. Cell, 2020. 183(3): p. 786801 e19.

190. Machiels, B., et al., A gammaherpesvirus provides protection against allergic asthma by inducing the replacement of resident alveolar macrophages with regulatory monocytes. Nat Immunol, 2017. 18(12): p. 1310-1320.

191. Yao, Y., et al., Induction of Autonomous Memory Alveolar Macrophages Requires T Cell Help and Is Critical to Trained Immunity. Cell, 2018. 175(6): p. 1634-1650 e17.

192. Tsou, C.L., et al., Critical roles for CCR2 and MCP-3 in monocyte mobilization from bone marrow and recruitment to inflammatory sites. J Clin Invest, 2007. 117(4): p. 902-9.

193. Cushing, S.D., et al., Minimally modified low density lipoprotein induces monocyte chemotactic protein 1 in human endothelial cells and smooth muscle cells. Proc Natl Acad Sci U S A, 1990. 87(13): p. 5134-8. 
194. Strieter, R.M., et al., Monocyte chemotactic protein gene expression by cytokinetreated human fibroblasts and endothelial cells. Biochem Biophys Res Commun, 1989. 162(2): p. 694-700.

195. Küper, C., F.X. Beck, and W. Neuhofer, Autocrine MCP-1/CCR2 signaling stimulates proliferation and migration of renal carcinoma cells. Oncol Lett, 2016. 12(3): p. 2201-2209.

196. Gerszten, R.E., et al., MCP-1 and IL-8 trigger firm adhesion of monocytes to vascular endothelium under flow conditions. Nature, 1999. 398(6729): p. 718-23.

197. Bakos, E., et al., CCR2 Regulates the Immune Response by Modulating the Interconversion and Function of Effector and Regulatory T Cells. J Immunol, 2017. 198(12): p. 4659-4671.

198. Kurihara, T., et al., Defects in macrophage recruitment and host defense in mice lacking the CCR2 chemokine receptor. J Exp Med, 1997. 186(10): p. 1757-62.

199. Kuziel, W.A., et al., Severe reduction in leukocyte adhesion and monocyte extravasation in mice deficient in CC chemokine receptor 2. Proc Natl Acad Sci U S A, 1997. 94(22): p. 12053-8.

200. Kratofil, R.M., P. Kubes, and J.F. Deniset, Monocyte Conversion During Inflammation and Injury. Arterioscler Thromb Vasc Biol, 2017. 37(1): p. 35-42.

201. Funamizu, N., et al., Macrophage migration inhibitory factor induces epithelial to mesenchymal transition, enhances tumor aggressiveness and predicts clinical outcome in resected pancreatic ductal adenocarcinoma. Int J Cancer, 2013. 132(4): p. 785-94. 
202. Denz, A., et al., Inhibition of MIF leads to cell cycle arrest and apoptosis in pancreatic cancer cells. J Surg Res, 2010. 160(1): p. 29-34.

203. Kalafati, L., et al., Innate Immune Training of Granulopoiesis Promotes Anti-tumor Activity. Cell, 2020. 183(3): p. 771-785 e12.

204. Lin, H., et al., Host expression of PD-L1 determines efficacy of PD-L1 pathway blockade-mediated tumor regression. J Clin Invest, 2018. 128(2): p. 805-815.

205. Nobre, C.C., et al., Macrophage Migration Inhibitory Factor (MIF): Biological Activities and Relation with Cancer. Pathol Oncol Res, 2017. 23(2): p. 235-244.

206. Noe, J.T. and R.A. Mitchell, MIF-Dependent Control of Tumor Immunity. Front Immunol, 2020. 11: p. 609948.

207. Crișan, T.O., M.G. Netea, and L.A. Joosten, Innate immune memory: Implications for host responses to damage-associated molecular patterns. Eur J Immunol, 2016. 46(4): p. 817-28.

208. Bekkering, S., et al., In Vitro Experimental Model of Trained Innate Immunity in Human Primary Monocytes. Clin Vaccine Immunol, 2016. 23(12): p. 926-933.

209. Luo, Y., et al., Interleukin-10 inhibits Mycobacterium bovis bacillus CalmetteGuérin (BCG)-induced macrophage cytotoxicity against bladder cancer cells. Clin Exp Immunol, 2010. 160(3): p. 359-68.

210. Luo, Y., et al., Role of Th1-stimulating cytokines in bacillus Calmette-Guérin (BCG)-induced macrophage cytotoxicity against mouse bladder cancer MBT-2 cells. Clin Exp Immunol, 2006. 146(1): p. 181-8. 
211. Luo, Y. and M.J. Knudson, Mycobacterium bovis bacillus Calmette-Guérininduced macrophage cytotoxicity against bladder cancer cells. Clin Dev Immunol, 2010. 2010: p. 357591.

212. Lesokhin, A.M., et al., Monocytic CCR2(+) myeloid-derived suppressor cells promote immune escape by limiting activated CD 8 T-cell infiltration into the tumor microenvironment. Cancer Res, 2012. 72(4): p. 876-86.

213. Nywening, T.M., et al., Targeting both tumour-associated CXCR2(+) neutrophils and CCR2(+) macrophages disrupts myeloid recruitment and improves chemotherapeutic responses in pancreatic ductal adenocarcinoma. Gut, 2018. 67(6): p. 1112-1123.

214. Sanford, D.E., et al., Inflammatory monocyte mobilization decreases patient survival in pancreatic cancer: a role for targeting the CCL2/CCR2 axis. Clin Cancer Res, 2013. 19(13): p. 3404-15.

215. Nywening, T.M., et al., Targeting tumour-associated macrophages with CCR2 inhibition in combination with FOLFIRINOX in patients with borderline resectable and locally advanced pancreatic cancer: a single-centre, open-label, dose-finding, non-randomised, phase 1 b trial. Lancet Oncol, 2016. 17(5): p. 651-62.

216. Pahler, J.C., et al., Plasticity in tumor-promoting inflammation: impairment of macrophage recruitment evokes a compensatory neutrophil response. Neoplasia, 2008. 10(4): p. 329-40.

217. Ugel, S., et al., Tumor-induced myeloid deviation: when myeloid-derived suppressor cells meet tumor-associated macrophages. J Clin Invest, 2015. 125(9): p. $3365-76$. 
218. Ye, C., L. Zheng, and C.H. Yuan, [Pancreatic ductal adenocarcinoma immune microenvironment and immunotherapy prospects]. Zhonghua Wai Ke Za Zhi, 2019. 57(1): p. 10-15.

219. Leinwand, J. and G. Miller, Regulation and modulation of antitumor immunity in pancreatic cancer. Nat Immunol, 2020. 21(10): p. 1152-1159.

220. Haas, L. and A.C. Obenauf, Allies or Enemies-The Multifaceted Role of Myeloid Cells in the Tumor Microenvironment. Front Immunol, 2019. 10: p. 2746.

221. Panni, R.Z., et al., Agonism of CD11b reprograms innate immunity to sensitize pancreatic cancer to immunotherapies. Sci Transl Med, 2019. 11(499).

222. Liu, X., G.D. Hogg, and D.G. DeNardo, Rethinking immune checkpoint blockade: 'Beyond the T cell'. J Immunother Cancer, 2021. 9(1).

223. Orr, B. and R.P. Edwards, Diagnosis and Treatment of Ovarian Cancer. Hematol Oncol Clin North Am, 2018. 32(6): p. 943-964.

224. Sugarbaker, P.H., Intraperitoneal delivery of chemotherapeutic agents for the treatment of peritoneal metastases: current challenges and how to overcome them. Expert Opin Drug Deliv, 2019. 16(12): p. 1393-1401.

225. Jaaback, K. and N. Johnson, Intraperitoneal chemotherapy for the initial management of primary epithelial ovarian cancer. Cochrane Database Syst Rev, 2006(1): p. Cd005340.

226. Karakas, Y., S. Lacin, and S. Yalcin, Recent advances in the management of pancreatic adenocarcinoma. Expert Rev Anticancer Ther, 2018. 18(1): p. 51-62.

227. Grossberg, A.J., et al., Multidisciplinary standards of care and recent progress in pancreatic ductal adenocarcinoma. CA Cancer J Clin, 2020. 70(5): p. 375-403. 
228. Saijo, S., et al., Dectin-1 is required for host defense against Pneumocystis carinii but not against Candida albicans. Nat Immunol, 2007. 8(1): p. 39-46.

229. Bernhagen, J., et al., Purification, bioactivity, and secondary structure analysis of mouse and human macrophage migration inhibitory factor $(M I F)$. Biochemistry, 1994. 33(47): p. 14144-55.

230. Chen, T.J. and N. Kotecha, Cytobank: providing an analytics platform for community cytometry data analysis and collaboration. Curr Top Microbiol Immunol, 2014. 377: p. 127-57.

231. Nowicka, M., et al., CyTOF workflow: differential discovery in high-throughput high-dimensional cytometry datasets. F1000Res, 2017. 6: p. 748.

232. Wilkerson, M.D. and D.N. Hayes, ConsensusClusterPlus: a class discovery tool with confidence assessments and item tracking. Bioinformatics, 2010. 26(12): p. 1572-3.

233. Finak G, J.M., FlowWorkspace: Infrastructure for representing and interacting with gated and ungated cytometry data sets. . Bioconductor Version, 2019(Release(3.9)).

234. Finak, G., W. Jiang, and R. Gottardo, CytoML for cross-platform cytometry data sharing. Cytometry A, 2018. 93(12): p. 1189-1196.

235. Z. V. Crowell H, C.S., Robinson M CATALYST: Cytometry Data Analysis Tools. $R$

package version 1.10.3, https://github.com/HelenaLC/CATALYST,. 2020. 
236. Cock, P.J., et al., The Sanger FASTQ file format for sequences with quality scores, and the Solexa/Illumina FASTQ variants. Nucleic Acids Res, 2010. 38(6): p. 176771.

237. Illumina, BaseSpace User Guide. 2014.

238. Andrews, S. FastQC: A Quality Control Tool for High Throughput Sequence Data. 2015.

239. Dobin, A., et al., STAR: ultrafast universal RNA-seq aligner. Bioinformatics, 2013. 29(1): p. 15-21.

240. Love, M., W. Huber, and S. Anders, Moderated estimation of fold change and dispersion for RNA-seq data with DESeq2. 2014, bioRxiv.

241. Trapnell, C., et al., Differential gene and transcript expression analysis of RNA-seq experiments with TopHat and Cufflinks. Nat Protoc, 2012. 7(3): p. 562-78.

242. Anders, S., P.T. Pyl, and W. Huber, HTSeq--a Python framework to work with highthroughput sequencing data. Bioinformatics, 2015. 31(2): p. 166-9.

243. Boyle, E.I., et al., GO::TermFinder--open source software for accessing Gene Ontology information and finding significantly enriched Gene Ontology terms associated with a list of genes. Bioinformatics, 2004. 20(18): p. 3710-5.

244. Kanehisa, M. and S. Goto, KEGG: kyoto encyclopedia of genes and genomes. Nucleic Acids Res, 2000. 28(1): p. 27-30.

245. Andrews, S. FastQC: A Quality Control Tool for High Throughput Sequence Data. 2015; Available from: http://www.bioinformatics.babraham.ac.uk/projects/fastqc/. 
246. Stuart, T., et al., Comprehensive integration of single-cell data. Cell, 2019. 177(7): p. $1888-1902$. e21.

247. Lun, A.T., et al., EmptyDrops: distinguishing cells from empty droplets in dropletbased single-cell RNA sequencing data. Genome biology, 2019. 20(1): p. 1-9.

248. Hafemeister, C. and R. Satija, Normalization and variance stabilization of singlecell RNA-seq data using regularized negative binomial regression. Genome biology, 2019. 20(1): p. 1-15.

249. Becht, E., et al., Dimensionality reduction for visualizing single-cell data using UMAP. Nature biotechnology, 2019. 37(1): p. 38-44.

250. Diaz-Mejia, J.J., et al., Evaluation of methods to assign cell type labels to cell clusters from single-cell RNA-sequencing data. F1000Research, 2019. 8.

251. Hänzelmann, S., R. Castelo, and J. Guinney, GSVA: gene set variation analysis for microarray and RNA-seq data. BMC bioinformatics, 2013. 14(1): p. 7.

252. Finak, G., et al., MAST: a flexible statistical framework for assessing transcriptional changes and characterizing heterogeneity in single-cell RNA sequencing data. Genome biology, 2015. 16(1): p. 1-13.

253. Suffiotti, M., et al., Identification of innate lymphoid cells in single-cell RNA-Seq data. Immunogenetics, 2017. 69(7): p. 439-450.

254. Entwistle, L.J., et al., Pulmonary Group 2 Innate Lymphoid Cell Phenotype Is Context Specific: Determining the Effect of Strain, Location, and Stimuli. Frontiers in Immunology, 2020. 10(3114).

255. Ricardo-Gonzalez, R.R., et al., Tissue signals imprint ILC2 identity with anticipatory function. Nat Immunol, 2018. 19(10): p. 1093-1099. 
256. Zilionis, R., et al., Single-Cell Transcriptomics of Human and Mouse Lung Cancers Reveals Conserved Myeloid Populations across Individuals and Species. Immunity, 2019. 50(5): p. 1317-1334.e10. 
MATERIALS

\begin{tabular}{|c|c|c|}
\hline REAGENT or RESOURCE & SOURCE & IDENTIFIER \\
\hline \multicolumn{3}{|l|}{ Antibodies } \\
\hline Viability Dye & Invitrogen & Ref \#: 65-0865-14 \\
\hline Anti-mouse CD45, 30-F11 & BioLegend & $\begin{array}{l}\text { Cat \# } 103129 \\
\text { RRID: Ab_893343 }\end{array}$ \\
\hline Anti-mouse CD11b, M1/70 & BioLegend & $\begin{array}{l}\text { Cat \# 101215 } \\
\text { RRID:AB_312798 }\end{array}$ \\
\hline Anti-mouse F4/80, BM8 & BioLegend & $\begin{array}{l}\text { Cat \# } 123115 \\
\text { RRID: AB_893493 }\end{array}$ \\
\hline Anti-mouse CD3, 17A2 & BioLegend & $\begin{array}{l}\text { Cat \# } 100203 \\
\text { RRID:AB_312660 }\end{array}$ \\
\hline Anti-mouse CD4, GK1.5 & BioLegend & $\begin{array}{l}\text { Cat \# 100411 } \\
\text { RRID:AB_312696 }\end{array}$ \\
\hline Anti-mouse CD8, 53-6.7 & BioLegend & $\begin{array}{l}\text { Cat \# 100721 } \\
\text { RRID:AB_312760 }\end{array}$ \\
\hline Anti-mouse CD19, 6D5 & BioLegend & $\begin{array}{l}\text { Cat \# 115507 } \\
\text { RRID:AB_313642 }\end{array}$ \\
\hline Anti-mouse NK1.1, PK136 & BioLegend & $\begin{array}{l}\text { Cat \# } 108709 \\
\text { RRID:AB_313396 }\end{array}$ \\
\hline Anti-mouse Ly6C, HK1.4 & BioLegend & $\begin{array}{l}\text { Cat \# 128005 } \\
\text { RRID:AB_1186134 }\end{array}$ \\
\hline Anti-mouse Ly6G, 1A8 & BioLegend & $\begin{array}{l}\text { Cat \# } 127607 \\
\text { RRID:AB_1186104 }\end{array}$ \\
\hline Anti-mouse CCR2, 475301 & R\&D Systems & $\begin{array}{l}\text { Cat \# FAB5538P } \\
\text { RRID:AB_1071841 } \\
4\end{array}$ \\
\hline Anti-mouse TNF $\alpha$, MP6-XT22 & BioLegend & $\begin{array}{l}\text { Cat \# 506305 } \\
\text { RRID:AB_315426 }\end{array}$ \\
\hline Anti-mouse IFN $\gamma, \mathrm{XMG} 1.2$ & BioLegend & $\begin{array}{l}\text { Cat \# 505807 } \\
\text { RRID:AB_315401 }\end{array}$ \\
\hline Anti-Rat IgG1, $\kappa$ isotype, RTK2071 & BioLegend & $\begin{array}{l}\text { Cat \# 400407 } \\
\text { RRID:AB_326513 }\end{array}$ \\
\hline Anti-Rat IgG2b, $\kappa$ isotype, RTK4530 & BioLegend & $\begin{array}{l}\text { Cat \# 400607 } \\
\text { RRID:AB_326551 }\end{array}$ \\
\hline Anti-mouse CD8, 53-6.7 & BioLegend & $\begin{array}{l}\text { Cat \# } 100755 \\
\text { RRID:AB_2562796 }\end{array}$ \\
\hline Anti-mouse CD3, 145-2C11 & BioLegend & $\begin{array}{l}\text { Cat \# } 100345 \\
\text { RRID:AB_2563748 }\end{array}$ \\
\hline
\end{tabular}




\begin{tabular}{|c|c|c|}
\hline Anti-mouse CD4, RM4-5 & BioLegend & $\begin{array}{l}\text { Cat \# } 100561 \\
\text { RRID:AB_2562762 }\end{array}$ \\
\hline Anti-mouse CD11b, M1/70 & BioLegend & $\begin{array}{l}\text { Cat \# } 101249 \\
\text { RRID:AB_2562797) }\end{array}$ \\
\hline Anti-mouse Ly6C, HK1.4 & BioLegend & $\begin{array}{l}\text { Cat \# } 128039 \\
\text { RRID:AB_2563783 }\end{array}$ \\
\hline Anti-mouse CD19, 6D5 & BioLegend & $\begin{array}{l}\text { Cat \# } 115547 \\
\text { RRID:AB_2562806 }\end{array}$ \\
\hline Anti-mouse IFN $\gamma, \mathrm{XMG} 1.2$ & BioLegend & $\begin{array}{l}\text { Cat \# 505843 } \\
\text { RRID:AB_2562847 }\end{array}$ \\
\hline Anti-mouse IL-12/IL-35 p53, 27537 & R+D Systems & $\begin{array}{l}\text { Cat \# MAB } 1570 \\
\text { RRID:AB_2295829 }\end{array}$ \\
\hline Anti-mouse IL-17, TC11-18H10.1 & BioLegend & $\begin{array}{l}\text { Cat \# 506935 } \\
\text { RRID:AB_2562850 }\end{array}$ \\
\hline Anti-mouse CCR2, 475301R & R+D Systems & Cat \# MAB55381R \\
\hline Anti-mouse Granzyme B, 12F9B65 & BioLegend & $\begin{array}{l}\text { Cat \# 662801 } \\
\text { RRID:AB_2564373 }\end{array}$ \\
\hline Anti-mouse TCR $\gamma \delta$, GL3 & BioLegend & $\begin{array}{l}\text { Cat \# } 118101 \\
\text { RRID:AB_313826 }\end{array}$ \\
\hline Anti-mouse Tbet, 4B10 & BioLegend & $\begin{array}{l}\text { Cat \# 644825 } \\
\text { RRID:AB_2563788 }\end{array}$ \\
\hline Anti-mouse CD107a, 1D4B & BioLegend & $\begin{array}{l}\text { Cat \# 328635 } \\
\text { RRID:AB_2563708 }\end{array}$ \\
\hline Anti-mouse TNF $\alpha$ (141Pr), MP6-XT22 & Fluidigm & Product \# 3141012B \\
\hline Anti-mouse CD11c (142 Nd), N418 & Fluidigm & Product \# 3142003B \\
\hline Anti-mouse IL-2 (144Nd), JES6-5H4 & Fluidigm & Product \# 3144002B \\
\hline Anti-mouse CD69 (145 Nd), H.2F3 & Fluidigm & Product \#3145005B \\
\hline Anti-mouse F4/80 (146 Nd), BM8 & Fluidigm & Product \#3146008B \\
\hline Anti-mouse CD44 (150Nd), IM7 & Fluidigm & Product \#3150018B \\
\hline Anti-mouse Ly-6G (151 Eu), IA8 & Fluidigm & Product \#3151010B \\
\hline $\begin{array}{l}\text { Anti-mouse CD274 (PD-L1) (153 Eu), } \\
\text { 10F.9G2 }\end{array}$ & Fluidigm & Product \#3153016B \\
\hline Anti-mouse FoxP3 (158 Gd), FJK-16S & Fluidigm & Product \#3165024A \\
\hline $\begin{array}{l}\text { Anti-mouse CD279 (PD-1) (159 Gd), } \\
\text { 29F.1A12 }\end{array}$ & Fluidigm & Product \#3159024B \\
\hline $\begin{array}{l}\text { Anti-mouse CD62L (L-Selectin) (160 Gd), } \\
\text { MEL-14 }\end{array}$ & Fluidigm & Product \#3160008B \\
\hline Anti-mouse iNOS (161Dy), CXNFT & Fluidigm & Product \#3161011B \\
\hline Anti-mouse CX3CR1 (164DY), SA011F11 & Fluidigm & Product \#3164023B \\
\hline Anti-mouse IL-6 (167Er), MP5-20F3 & Fluidigm & Product \#3167003B \\
\hline Anti-mouse CD206 (169Tm), C089C3 & Fluidigm & Product \#3169021B \\
\hline Anti-mouse C161 (NK1.1)(170Er), PK136 & Fluidigm & Product \#3170002B \\
\hline Anti-mouse CD80 (171Yb), 16-10A1 & Fluidigm & Product \#3171008B \\
\hline Anti-mouse CD86 (172Yb), GL1 & Fluidigm & Product \#3172016B \\
\hline $\begin{array}{l}\text { Anti-mouse CD223 (LAG-3) (174Yb), } \\
\text { C9B7W }\end{array}$ & Fluidigm & Product \#3174019B \\
\hline
\end{tabular}




\begin{tabular}{|c|c|c|}
\hline Anti-mouse I-A/I-E (209Bi), M5/114.15 & Fluidigm & Product \#3209006B \\
\hline Anti-mouse CD45 (89Y), 30-F11 & Fluidigm & Product \#3089005B \\
\hline InVivoMAb anti-mouse Anti Ly6G, 1A8 & Bio X Cell & $\begin{array}{l}\text { BE0075-1 } \\
\text { RRID:AB_1107721 }\end{array}$ \\
\hline Rat IgG2a Isotype Control, 2A3 & Bio X Cell & $\begin{array}{l}\text { BE0089 } \\
\text { RRID:AB_1107769 }\end{array}$ \\
\hline $\begin{array}{l}\text { InVivoMAb anti-mouse PD-L1(B7- } \\
\text { H1(10F.9G2) }\end{array}$ & Bio X Cell & $\begin{array}{l}\text { BE0101 } \\
\text { RRID:AB_1094907 } \\
3\end{array}$ \\
\hline Rat IgG2b Isotype Control (LTF-2) & Bio $\mathrm{X}$ cell & $\begin{array}{l}\text { BE0090 } \\
\text { RRID:AB_1107780 }\end{array}$ \\
\hline Anti Mouse anti NK1.1 mAb PK136 & $\begin{array}{l}\text { Made in the } \\
\text { Lab of Dr. Jun } \\
\text { Yan, } \\
\text { University of } \\
\text { Louisville }\end{array}$ & \\
\hline Anti Mouse anti CD4 mAb GK1.5 & $\begin{array}{l}\text { Made in the } \\
\text { Lab of Dr. Jun } \\
\text { Yan, } \\
\text { University of } \\
\text { Louisville }\end{array}$ & \\
\hline Anti Mouse anti CD8 mAb 53-6.72 & $\begin{array}{l}\text { Made in the } \\
\text { Lab of Dr. Jun } \\
\text { Yan, } \\
\text { University of } \\
\text { Louisville }\end{array}$ & \\
\hline \multicolumn{3}{|c|}{ Chemicals, Peptides, and Recombinant Proteins } \\
\hline $\begin{array}{l}\text { Tris(2-carboxyethyl)phosphine } \\
\text { hydrochloride (TCEP) solution, pH } 7.0 \\
(10 x 1 \mathrm{~mL}, .5 \mathrm{M})\end{array}$ & $\begin{array}{l}\text { MilliporeSig } \\
\text { ma }\end{array}$ & 626547 \\
\hline HRP-protector peroxidase stabilizer & $\begin{array}{l}\text { Boca } \\
\text { Scientific }\end{array}$ & 222050 \\
\hline Antibody Stabilizer PBS & $\begin{array}{l}\text { Boca } \\
\text { Scientific }\end{array}$ & 131050 \\
\hline Sodium Azide, BioUltra, >99.5\%(T) & $\begin{array}{l}\text { MilliporeSig } \\
\text { ma }\end{array}$ & 71289 \\
\hline $\begin{array}{l}\text { 5-([4,6-Dichlorotriazin-2- } \\
\text { yl]amino)fluorescein hydrochloride } \\
\text { (DTAF) }\end{array}$ & $\begin{array}{l}\text { Sigma- } \\
\text { Aldrich }\end{array}$ & D0531 \\
\hline ACK & $\begin{array}{l}\text { Thermo } \\
\text { Fisher } \\
\text { Scientific }\end{array}$ & A1049201 \\
\hline Brefeldin A & Biolegend & 420601 \\
\hline Collagenase I & Sigma & C9891 \\
\hline DNase & Sigma & D5025 \\
\hline
\end{tabular}




\begin{tabular}{|c|c|c|}
\hline Endotoxin-free DPBS & $\begin{array}{l}\text { Sigma- } \\
\text { Aldrich }\end{array}$ & TMS-012-A \\
\hline Hyaluronidase & Sigma & H2126 \\
\hline $\begin{array}{l}\text { Matrigel }{ }^{\circledR} \text { Basement Membrane Matrix, } \\
\text { LDEV-free, } 5 \mathrm{~mL}\end{array}$ & Corning & 356234 \\
\hline Ionomycin Calcium Salt & $\begin{array}{l}\text { Sigma- } \\
\text { Aldrich }\end{array}$ & I0634 \\
\hline PMA & $\begin{array}{l}\text { Sigma- } \\
\text { Aldrich }\end{array}$ & 79346 \\
\hline Fixation Buffer & BioLegend & 420801 \\
\hline Permeabilization buffer & BioLegend & 421002 \\
\hline $\begin{array}{l}\text { Fluoresbrite }{ }^{\circledR} \text { YG Microspheres, } \\
\text { Calibration Grade } 3.00 \mu \mathrm{m}\end{array}$ & Polysciences & $18861-1$ \\
\hline $3.00 \mu \mathrm{m}$ polystyrene Miroparticle & $\begin{array}{l}\text { Sigma- } \\
\text { Aldrich }\end{array}$ & 79166 \\
\hline MACS Running Buffer & $\begin{array}{l}\text { Miltenyi } \\
\text { Biotec }\end{array}$ & $130-091-221$ \\
\hline HEPES & Corning & 25-060-CI \\
\hline TRIzol & Invitrogen & 15596018 \\
\hline Poly-L-Lysine & $\begin{array}{l}\text { Advanced } \\
\text { BioMatrix }\end{array}$ & 5048 \\
\hline $\begin{array}{l}\text { XenoLight D-Luciferin }- \text { K+ Salt } \\
\text { Bioluminescent Substrate }\end{array}$ & Perkin Elmer & 12799 \\
\hline CD11b MicroBeads, Human and mouse & $\begin{array}{l}\text { Miltenyi } \\
\text { Biotec }\end{array}$ & 130-049-601 \\
\hline N-Acetyl-l-cysteine (NAC) & $\begin{array}{l}\text { Sigma- } \\
\text { Aldrich }\end{array}$ & A7250-5G \\
\hline Lipopolysaccharides from E.coli0111:B4 & Sigma & L4391-1MG \\
\hline $\begin{array}{l}.25 \% \text { Trypsin } 2.21 \text { mM EDTA, 1x [-] } \\
\text { sodium bicarbonate }\end{array}$ & Corning & $25-053-\mathrm{Cl}$ \\
\hline FBS & $\begin{array}{l}\text { Atlanta } \\
\text { Biologicals }\end{array}$ & Cat \#: S11150 \\
\hline $\begin{array}{l}\text { Bovine Serum Albumin Cohn Fraction V } \\
\text { Powder }\end{array}$ & $\begin{array}{l}\text { Equitech0Bio, } \\
\text { Inc. }\end{array}$ & $\begin{array}{l}\text { Cat \# BAC62-1000 } \\
\text { Lot \#171130-0262 }\end{array}$ \\
\hline Buprenorphine, hydrochloride, Injection & $\begin{array}{l}\text { Par } \\
\text { Pharmaceutic } \\
\text { al }\end{array}$ & 10153802 \\
\hline p-SCN-Bn-Deferoxamine & Macrocyclics & B-705 \\
\hline sodium carbonate & VWR & BJ34277-1L \\
\hline Oxalic acid, anhydrous & Sigma & $756888-50 \mathrm{G}$ \\
\hline UltraPure $^{\mathrm{TM}} 1 \mathrm{M}$ Tris-HCI Buffer, $\mathrm{pH} 7.5$ & Invitrogen & 15567027 \\
\hline Zr-89 oxalate & $\begin{array}{l}\text { Washington } \\
\text { University at } \\
\text { St. Louis }\end{array}$ & $\begin{array}{l}\text { Radionuclide } \\
\text { Processing } \\
\text { Laboratory }\end{array}$ \\
\hline Dulbecco's Phosphate Buffered Saline & Sigma & D8537 \\
\hline Chloroform & Sigma & $\mathrm{C} 2432$ \\
\hline
\end{tabular}




\begin{tabular}{|c|c|c|}
\hline 2-propanol & $\begin{array}{l}\text { Fisher } \\
\text { Scientific }\end{array}$ & A41701 \\
\hline UltaPure ${ }^{\mathrm{TM}}$ Glycogen & $\begin{array}{l}\text { Thermo } \\
\text { Fisher } \\
\text { Scientific }\end{array}$ & 10814010 \\
\hline iScript ${ }^{\mathrm{TM}}$ cDNA Synthesis Kit & BioRad & 1708891 \\
\hline $\mathrm{iQ}^{\mathrm{TM}}$ SYBR $®$ Green Supermix & BioRad & 1708880 \\
\hline RPMI-1640 medium & Sigma & R8758-1L \\
\hline DMEM- high glucose & Sigma & D5796-500ML \\
\hline \multicolumn{3}{|l|}{ Critical Commercial Assays } \\
\hline $\begin{array}{l}\text { MaxPar MCP9 Antibody Labeling Kit, } \\
\text { 106Cd }\end{array}$ & Fluidigm & Cat \# 201106A \\
\hline $\begin{array}{l}\text { MaxPar MCP9 Antibody Labeling Kit, } \\
\text { 111Cd }\end{array}$ & Fluidigm & Cat \# 201111A \\
\hline $\begin{array}{l}\text { MaxPar MCP9 Antibody Labeling Kit, } \\
112 \mathrm{Cd}\end{array}$ & Fluidigm & Cat \# 201112A \\
\hline $\begin{array}{l}\text { MaxPar MCP9 Antibody Labeling Kit, } \\
\text { 113Cd }\end{array}$ & Fluidigm & Cat \# 201113A \\
\hline $\begin{array}{l}\text { MaxPar MCP9 Antibody Labeling Kit, } \\
\text { 114Cd }\end{array}$ & Fluidigm & Cat \# 201114A \\
\hline $\begin{array}{l}\text { MaxPar MCP9 Antibody Labeling Kit, } \\
\text { 116Cd }\end{array}$ & Fluidigm & Cat \# 201116A \\
\hline MaxPar X8 Antibody Labeling Kit, 146Nd & Fluidigm & Cat \# 201146A \\
\hline MaxPar X8 Antibody Labeling Kit, 149 Sm & Fluidigm & Cat \# 201149A \\
\hline MaxPar X8 Antibody Labeling Kit, 154 Sm & Fluidigm & Cat \# 201154A \\
\hline MaxPar X8 Antibody Labeling Kit, 156 Gd & Fluidigm & Cat \# 201156A \\
\hline MaxPar X8 Antibody Labeling Kit, 162 Dy & Fluidigm & Cat \# 201162A \\
\hline MaxPar X8 Antibody Labeling Kit, 165 Ho & Fluidigm & Cat \# 201165A \\
\hline MaxPar X8 Antibody Labeling Kit, 166 Er & Fluidigm & Cat \# 201166A \\
\hline MaxPar X8 Antibody Labeling Kit, 168Er & Fluidigm & Cat \# 201168A \\
\hline MaxPar X8 Antibody Labeling Kit, 173Yb & Fluidigm & Cat \# 201173A \\
\hline ELISA MAX ${ }^{\mathrm{TM}}$ standard set Mouse TNF- $\alpha$ & BioLegend & 430901 \\
\hline ELISA MAX ${ }^{\mathrm{TM}}$ standard set Mouse IL-6 & BioLegend & 431301 \\
\hline Mouse MIF DuoSet ELISA & R\&D Systems & DY1978 \\
\hline $\begin{array}{l}\text { Invitrogen } \mathrm{pHrodo}^{\mathrm{TM}} \text { Green S. aureus } \\
\text { BioParticles }{ }^{\mathrm{TM}} \text { Phagocytosis Kit for Flow } \\
\text { cytometry }\end{array}$ & $\begin{array}{l}\text { Thermo } \\
\text { Fisher } \\
\text { Scientific }\end{array}$ & P535367 \\
\hline Amylase Activity Assay Kit & $\begin{array}{l}\text { Millipore } \\
\text { Sigma }\end{array}$ & MAK009 \\
\hline QIAGEN RNAeasy Kit & QIAGEN & 74104 \\
\hline $\begin{array}{l}\text { Promega Luciferase Assay Systems } \\
\text { Luciferase Assay w/ Reporter Lysis buffer }\end{array}$ & Promega & E4030 \\
\hline $\begin{array}{l}\text { Chromium Next GEM Single Cell 3' GEM, } \\
\text { Library \& Gel Bead Kit v3.1, } 4 \text { rxns }\end{array}$ & $\begin{array}{l}10 \mathrm{X} \\
\text { Genomics }\end{array}$ & PN-1000128 \\
\hline MiSeq Reagent Micro Kit V2 300 cycles & Illumina & MS-102-2002 \\
\hline
\end{tabular}




\begin{tabular}{|c|c|c|}
\hline $\begin{array}{l}\text { NextSeq } 500 \text { High Output Kit v2.5 (150 } \\
\text { cycles) }\end{array}$ & Illumina & 20024907 \\
\hline \multicolumn{3}{|l|}{ Experimental Models: Cell Lines } \\
\hline KPC Cell Line & Ximbio & 153474 \\
\hline Pan02 Cell Line & $\begin{array}{l}\text { Gift from Dr. } \\
\text { Yong Lu at } \\
\text { Wake Forest } \\
\text { University }\end{array}$ & \\
\hline KPC/FC1242 Luc/GFP clone 3 & $\begin{array}{l}\text { Gift from Dr. } \\
\text { Michael } \\
\text { Dwinell, } \mathrm{PhD} \\
\text { at the Medical } \\
\text { College of } \\
\text { Wisconsin }\end{array}$ & \\
\hline \multicolumn{3}{|l|}{ Experimental Models: Organisms/Strains } \\
\hline Mouse: C57BL/6J & $\begin{array}{l}\text { The Jackson } \\
\text { Laboratory }\end{array}$ & 000664 \\
\hline Mouse: B6.129S4-Ccr2 ${ }^{\operatorname{tm} 11 f f} / \mathrm{J}\left(\mathrm{CCR} 2^{-l-}\right)$ & $\begin{array}{l}\text { The Jackson } \\
\text { Laboratory }\end{array}$ & 004999 \\
\hline Mouse: Dectin-1 ${ }^{-/}$ & & (Saijo et al, 2007) \\
\hline $\begin{array}{l}\text { Mouse: NOD.Cg- } \\
\text { Prkdc }{ }^{\text {scid }} I l 2 \mathrm{rg}^{t m l}{ }^{\mathrm{Wjl}} / \mathrm{SzJ} \text { (NSG) }\end{array}$ & $\begin{array}{l}\text { The Jackson } \\
\text { Laboratory }\end{array}$ & 005557 \\
\hline $\begin{array}{l}\text { Mouse: B6(Cg)-Ty } r^{c-2 J} / \mathrm{J} \text { (Albino } \\
\text { C57BL/6J mice) }\end{array}$ & $\begin{array}{l}\text { The Jackson } \\
\text { Laboratory }\end{array}$ & 000058 \\
\hline $\begin{array}{l}\text { Mouse: C57BL/6-CD45.1 B6.SJL- } \\
\text { ptprc }{ }^{a} P e p c^{b} / \text { BoyJ }\end{array}$ & $\begin{array}{l}\text { The Jackson } \\
\text { Laboratory }\end{array}$ & 002014 \\
\hline \multicolumn{3}{|l|}{ Oligonucleotides } \\
\hline $\begin{array}{l}\text { Primers for IL-6 } \\
\text { Forward: } \\
\text { AAGACAAAGCCAGAGTCCTTCA } \\
\text { Reverse: AGAGCATTGGAAATTGGGGT }\end{array}$ & & \\
\hline $\begin{array}{l}\text { Primers for IL-10 } \\
\text { Forward: } \\
\text { AGTGGAGCAGGTGAAGAGTG } \\
\text { Reverse: TTCGGAGAGAGGTACAAACG }\end{array}$ & & \\
\hline $\begin{array}{l}\text { Primers for iNOS } \\
\text { Forward: } \\
\text { GGTGAAGGGACTGAGCTGTTA } \\
\text { Reverse: CAACGTTCTCCGTTCTCTTGC }\end{array}$ & & \\
\hline $\begin{array}{l}\text { Primers for TNF- } \alpha \\
\text { Forward: } \\
\text { TGTAGCCCACGTCGTAGCAAA } \\
\text { Reverse: } \\
\text { GCTGGCACCACTAGTTGGTTGT }\end{array}$ & & \\
\hline
\end{tabular}




\begin{tabular}{|c|c|c|}
\hline $\begin{array}{l}\text { Primers for CCL2: } \\
\text { Forward: GGTCCCTGTCATGCTTCTGG } \\
\text { Reverse: GCTGCTGGTGATCCTCTTGT }\end{array}$ & & \\
\hline \multicolumn{3}{|l|}{ Software and Algorithms } \\
\hline CyTOF Workflow & $\begin{array}{l}\text { Nowicka M., } \\
\text { et al., } 2019\end{array}$ & $\begin{array}{l}\text { https://f1000research } \\
\text { com/articles/6- } \\
748 / \mathrm{v} 4\end{array}$ \\
\hline Prism 7 & GraphPad & $\begin{array}{l}\text { https://www.graphpa } \\
\text { d.com/scientific- } \\
\text { software/prism/ } \\
\text { RRID: SCR_002798 }\end{array}$ \\
\hline FlowJo v 10.7.1 & TreeStar & $\begin{array}{l}\text { https://www.flowjo. } \\
\text { com/ } \\
\text { RRID: SCR_008520 }\end{array}$ \\
\hline FastQC (v0.11.7) & $\begin{array}{l}\text { Andrews, } \\
2015\end{array}$ & $\begin{array}{l}\text { http://www.bioinfor } \\
\text { matics.babraham.ac. } \\
\text { uk/projects/fastqc/ }\end{array}$ \\
\hline STAR (v2.6) & $\begin{array}{l}\text { Dobin et al., } \\
2013\end{array}$ & $\begin{array}{l}\text { https://github.com/al } \\
\text { exdobin/STAR/ }\end{array}$ \\
\hline HTSeq (v0.10.0) & $\begin{array}{l}\text { Anders et al., } \\
2015\end{array}$ & $\begin{array}{l}\text { https://pypi.org/proje } \\
\text { ct/HTSeq/ }\end{array}$ \\
\hline DESeq2 (v1.24.0) & $\begin{array}{l}\text { Love et al., } \\
2014\end{array}$ & $\begin{array}{l}\text { https://bioconductor. } \\
\text { org/packages/release } \\
\text { /bioc/html/DESeq2.h } \\
\text { tml }\end{array}$ \\
\hline GSEA & $\begin{array}{l}\text { Subramanian } \\
\text { et al., } 2005\end{array}$ & $\begin{array}{l}\text { https://www.gsea- } \\
\text { msigdb.org/gsea/ind } \\
\text { ex.jsp }\end{array}$ \\
\hline CellRanger (v3.1.0) & $\begin{array}{l}\text { Zheng et al., } \\
2017\end{array}$ & $\begin{array}{l}\text { https://support.10xge } \\
\text { nomics.com// }\end{array}$ \\
\hline Seurat (v3.1.1) & $\begin{array}{l}\text { Stuart et al., } \\
2019\end{array}$ & $\begin{array}{l}\text { https://cloud.r- } \\
\text { project.org/web/pack } \\
\text { ages/Seurat/index.ht } \\
\mathrm{ml}\end{array}$ \\
\hline UMAP & $\begin{array}{l}\text { Becht et al., } \\
2019\end{array}$ & \\
\hline scater (v1.12.2) & $\begin{array}{l}\text { McCarthy et } \\
\text { al., } 2017\end{array}$ & $\begin{array}{l}\text { https://bioconductor. } \\
\text { org/packages/release } \\
\text { /bioc/html/scater.ht } \\
\underline{\text { ml }}\end{array}$ \\
\hline DropletUtils (v1.4.3) & $\begin{array}{l}\text { Lun et al., } \\
2019\end{array}$ & $\begin{array}{l}\overline{\text { https://bioconductor. }} \\
\text { org/packages/release } \\
\text { lbioc/html/DropletUt } \\
\text { ils.html }\end{array}$ \\
\hline
\end{tabular}




\begin{tabular}{|c|c|c|}
\hline sctransfrom (v0.2.0) & $\begin{array}{l}\text { Hafemeister } \\
\text { and Satija, } \\
2019\end{array}$ & $\begin{array}{l}\frac{\text { https://github.com/C }}{\text { hristophH/sctransfor }} \\
\underline{\text { m }}\end{array}$ \\
\hline MAST (v1.10.0) & $\begin{array}{l}\text { Finak et al., } \\
2015\end{array}$ & $\begin{array}{l}\text { https://www.biocond } \\
\text { uctor.org/packages/r } \\
\text { elease/bioc/html/MA } \\
\text { ST.html }\end{array}$ \\
\hline \multicolumn{3}{|l|}{ Other } \\
\hline $\begin{array}{l}\text { Amicon Ultra-.5 centrifugal Filter Unit, } \\
.5 \mathrm{~mL} \text { V-bottom, 8-pack } 3 \mathrm{kDa}\end{array}$ & $\begin{array}{l}\text { Millipore } \\
\text { Sigma }\end{array}$ & UFC500308 (3 kDa) \\
\hline $\begin{array}{l}\text { Amicon Ulrta-.5 centrifugal Filter Unit, } \\
.5 \mathrm{~mL} \text { V-bottom, } 8 \text {-pack } 50 \mathrm{kDa}\end{array}$ & $\begin{array}{l}\text { Millipore } \\
\text { Sigma }\end{array}$ & UFC505008 \\
\hline $\begin{array}{l}\text { Amicon Ultra-. } 5 \text { centrifugal Filter Unit, } \\
.5 \mathrm{~mL} \text { V-bottom, 8-pack } 100 \mathrm{kDa}\end{array}$ & $\begin{array}{l}\text { Millipore } \\
\text { Sigma }\end{array}$ & UFC510008 \\
\hline Eppendorf protein LoBind Tubes, $1.5 \mathrm{~mL}$ & Eppendorf & 0224431081 \\
\hline $\begin{array}{l}\text { polyglycolic acid braided absorbable } 5 / 0 \\
\text { suture }\end{array}$ & CP Medical & $421 \mathrm{~A}$ \\
\hline silk braided nonabsorbable 5/0 suture & CP Medical & $682 \mathrm{~S}$ \\
\hline $\begin{array}{l}40 \mu \mathrm{m} \text { Nylon, Sterile, DNase/RNase Free } \\
\text { Cell Strainers }\end{array}$ & VWR & $10199-655$ \\
\hline
\end{tabular}




\section{CURRICULUM VITAE}

NAME:

CONTACT:

DOB:

EDUCATION

\& TRAINING:
Anne Elena Geller

University of Louisville School of Medicine

Clinical and Translational Research Building 505 S Hancock St

Suite $327 \mathrm{H}$

Louisville, KY 40204

412-215-1868 | Ageller17@gmail.com

Pittsburgh, PA USA - November 6, 1991

M.D.

University of Louisville School of Medicine Louisville, KY USA

2015-present

Ph.D., Microbiology and Immunology

University of Louisville School of Medicine Louisville, KY USA

2017-2021

Intramural Research Fellow

National Institutes of Health

Bethesda, MD USA

2014-2015

M.S., Biomedical Sciences

Tufts University

Medford, MA USA

2013-2014

B.S., Biomedical Science in Engineering Minor in Engineering Entrepreneurship

University of Pennsylvania

Philadelphia, PA USA

2009-2013 
AWARDS \& HONORS:
Graduate Dean's Citation

"Awarded to one student from each department at commencement in recognition of superior accomplishment in their graduate studies" University of Louisville, 2021

American Association of Immunologists Travel Award Recipient to attend AAP/ASCI/APSA Meeting 2020

Nominated and Inducted into the Honor Society of Phi Kappa Phi for being in the top $10 \%$ of graduate student's GPA

2020

Research! Louisville Doctoral Basic Science Graduate Student Award, $3^{\text {rd }}$ Place 2018

Excellence in Bioengineering Senior Design Competition Award, University of Pennsylvania 2013

University of Pennsylvania, Dean's List 2011-2013

Pennsylvania Governor's School for the Sciences Scholar

2008
SCHOLARSHIPS \& FELLOWSHIPS
Integrated Programs in the Biomedical Sciences (IPIBS) Graduate Fellowship 2017-2019

Summer Research Scholars Program (SRSP) 2015, 2016

Physician-Scientist Training Program (M.D./Ph.D) Scholarship 2015-present

NIH Intramural Research Award Fellow 
PROFESSIONAL SOCIETIES:

\section{LEADERSHIP \& VOLUNTEER WORK}

American Association of Immunologists Student Member

2019-present

American Physician Scientists Association 2015-present

Greater Louisville Medical Society

2015-present
University of Louisville School of Medicine Strategic Planning Committee Member 2020

University of Louisville Faculty Forum Graduate Student Representative

2017-Present

Curriculum Committee Student Representative Department of Microbiology and Immunology 2017-2018

Class Treasurer for the Class of 2019 School of Medicine

2015-2016

Class Representative for the Biochemistry Course 2015

Volunteer at the EMW Women's Surgical Center Louisville, KY USA

2015-present

Medical Student Volunteer at the GLOH and HOPE community clinics

2015-2016

PUBLICATIONS: (*Co-first authorship)

Geller, A. and J. Yan, The Role of Membrane Bound Complement Regulatory Proteins 
in Tumor Development and Cancer Immunotherapy. Frontiers in Immunology, 2019. 10(1074).

*Geller, A., *Shrestha R, Yan J. Yeast-Derived $\beta$-Glucan in Cancer: Novel Uses of a Traditional Therapeutic. International Journal of Molecular Sciences. 2019; 20(15):3618. Woeste, M. R., Bhutiani, N., Geller, A. E., Eldridge-Hindy, H., McMasters, K. M., \& Ajkay, N. (2020). Identifying Factors Predicting Prolonged Opioid Use After Mastectomy. Ann Surg Oncol. doi: 10.1245/s10434-019-08171-4

Bridgewater C, Geller A, Hu X, Burlison JA, Zhang HG, Yan J, Guo H. 89Zr-Labeled Anti-PD-L1 Antibody Fragment for Evaluating In Vivo PD-L1 Levels in Melanoma Mouse Model. Cancer Biother Radiopharm. 2020 Oct;35(8):549-557. doi: 10.1089/cbr.2019.3056. Epub 2020 Apr 21.

Geller A, Yan J. Could the Induction of Trained Immunity by $\beta$-Glucan Serve as a Defense Against COVID-19?. Front Immunol. 2020;11:1782. Published 2020 Jul 14. doi:10.3389/fimmu.2020.01782

*Woeste, M.R., *Geller, A.E., Martin, R.C.G. et al. Optimizing the Combination of Immunotherapy and Trans-Arterial Locoregional Therapy for Stages B and C Hepatocellular Cancer. Ann Surg Oncol (2021).

*Morrissey, S.M., ${ }^{*}$ Geller, A.E. et al., Emergence of Low-density Inflammatory Neutrophils Correlates with Hypercoagulable State and Disease Severity in COVID-19 Patients. medRxiv, 2020: p. 2020.05.22.20106724.

(Accepted at JCI Insight)

Geller AE, Shrestha R, Guo H, Chuanlin D, Woeste MR, Andreeva K, Chariker JH, Zhou M, Tieri D, Watson CT, Mitchell R, Martin RCG, Rouchka EC, Yan J, The Induction of Trained Immunity in the Pancreas Incites Anti-tumor Activity in Models of Pancreatic Cancer (2021)

(Manuscript in preparation)

Noe JT, Rendon BE, Geller AE, Morrissey SM, Conroy LR, Kim EJ, Wise-Mitchell A, Rizzo MBS, Young LE, Bruntz RC, Affronti HC, McMaster KM, Clem BF, Wellen KE, Sun RC, Mitchell RA. Lactate Supports a Metabolic-Epigenetic Link in Macrophage Polarization (2021) (Manuscript in preparation) 
Ding C, Shrestha R, Zhu X, Geller AE, Woeste MR, Li W, Yuan F, Chariker JL, Hu X, Li H, Puckett A, Zhang H, Rouchka EC, Mitchell R, Siskind L, Zhang X, McMasters KM, Yu Y, Yan J. Induction of Trained Immunity Controls Cancer Metastasis Through the Sphingolipids-Mitochondrial Fission Pathway (2021)

(Manuscript currently under peer review)

\section{ABSTRACT AND POSTER PRESENTATIONS:}

A.E. Geller, H. Guo, C. Ding, J. Yan. "In Vitro and In Vivo Evaluation of Radiolabeled Particulate B-glucan.” Research Louisville, Louisville, KY. October 2016

A.E. Geller, H. Guo, C. Ding, J. Yan. "In Vitro and In Vivo Evaluation of Radiolabeled Particulate $\beta$-glucan.” Southeastern Medical Scientist Symposium, Atlanta, Georgia. November 2017

H. Guo, A.E. Geller, J. Yan. " ${ }^{69}$ Zr-Df-anti-PD-L1 for evaluating in vivo PD-L1 levels in mouse models lung cancer." Society of Nuclear Medicine and Molecular Imaging. Anaheim, California. June, 2018

A.E. Geller, H. Guo, C. Ding, J. Yan. “The characterization of $ß$-glucan's mechanism of action as an anti-cancer therapeutic." Research Louisville, Louisville, KY. October 2018

A.E. Geller, H. Guo, C. Ding, J. Yan. "ß-glucan as an anticancer agent in the scope of trained immunity: future implications for pancreatic cancer." American Physician Scientist Association Conference, Chicago, IL. April 2019

A.E. Geller, H. Guo, C. Ding, J. Yan. "ß-glucan as an anticancer agent in the scope of trained immunity: future implications for pancreatic cancer." National $\mathrm{MD} / \mathrm{PhD}$ Conference, Copper Mountain, CO. July 2019

A.E. Geller, H. Guo, C. Ding, J. Yan. "Harnessing the power of ß-Glucan in trained immunity, to treat and prevent pancreatic cancer." Research Louisville, Louisville, KY. October 2019

A.E. Geller, C Ding, H Guo and JYan. "Harnessing the power of trained immunity using yeast-derived B- Glucan in trained immunity to treat and prevent pancreatic cancer." Journal of Immunology, May 1 2020. American Association of Immunologists. Vol 204, Issue 1, Supplement 
Liang Liu, Anne Geller, Jun Yan and Haixun Guo. " ${ }^{89} \mathrm{Zr}$-labeled anti-Pd-1 antibody for in vivo monitoring of infiltrating PD-1 ${ }^{+} \mathrm{T}$ lymphocytes in a pancreatic tumor mouse model.” Journal of Nuclear Medicine, Vol 61, Issue supplement 1 May 1, 2020 